\title{
Self-consistent methods for structure and production of heavy and superheavy nuclei
}

\author{
G. G. Adamian ${ }^{1, a}$, N. V. Antonenko ${ }^{1,2}$, H. Lenske ${ }^{3}$, L. A. Malov ${ }^{1}$, Shan-Gui Zhou ${ }^{4,5,6,7}$ \\ ${ }^{1}$ Joint Institute for Nuclear Research, 141980 Dubna, Russia \\ 2 Tomsk Polytechnic University, 634050 Tomsk, Russia \\ ${ }^{3}$ Institut für Theoretische Physik der Justus-Liebig-Universität, D-35392 Gießen, Germany \\ ${ }^{4}$ CAS Key Laboratory of Theoretical Physics, Institute of Theoretical Physics, Chinese Academy of Sciences, Beijing 100190, China \\ ${ }^{5}$ School of Physical Sciences, University of Chinese Academy of Sciences, Beijing 100049, China \\ ${ }^{6}$ Center of Theoretical Nuclear Physics, National Laboratory of Heavy Ion Accelerator, Lanzhou 730000, China \\ ${ }^{7}$ Synergetic Innovation Center for Quantum Effects and Application, Hunan Normal University, Changsha 410081, China
}

Received: 2 September 2020 / Accepted: 18 January 2021 / Published online: 8 March 2021

(C) The Author(s), under exclusive licence to Società Italiana di Fisica and Springer-Verlag GmbH Germany, part of Springer Nature 2021

Communicated by Nicolas Alamanos

\begin{abstract}
Self-consistent methods for the structure of heavy and superheavy nuclei are reviewed. The construction and application of energy-density functionals are discussed. The relationship between the self-consistent methods and microscopic-macroscopic approaches is considered on the mean-field level. The extraction of single-particle potentials from the energy-density functional is described. The isotopic dependence of nucleon distributions and its influence on the nucleus-nucleus interaction is analyzed. As a new additional condition we introduce that the energy-density functional must describe the heights of the Coulomb barriers in nucleusnucleus interaction potentials, thus imposing constraints on the properties of the functional at low matter densities. The self-consistent methods are applied to describe the quasiparticle structure, cluster radioactivity, and fission of the heaviest nuclei. These methods are used to predict the probabilities of $\beta$-delayed multi-neutron emission and half-lives of $\beta$-decays and electron captures in heavy and superheavy nuclei. The predicted properties of superheavy nuclei are used to estimate the production cross-sections of superheavy nuclei in complete fusion reactions.
\end{abstract}

\section{Contents}

1 Introduction ................. 2

2 Overview of self-consistent nuclear-structure methods 3

3 The Giessen energy-density functional . . . . . . 6

3.1 Energy-density functionals and microscopic interactions . . . . . . . . . . . . 6

a e-mail: adamian@
3.2 Single-quasiparticle equations of motion . . . . 7

3.3 GEDF results for infinite nuclear matter and Fermi-liquid parameters . . . . . . . . . . 8

3.4 Pairing in infinite nuclear matter . . . . . . . 11

4 Reduction to a heuristic Schrödinger equation . . . . 12

4.1 Non-relativistic mean-field approach . . . . . . 12

4.2 Covariant mean-field approach . . . . . . . . 14

$4.3 \mathrm{EDF}$ for finite nuclei by inversion . . . . . 16

5 Including microscopic mean-field dynamics into the Mic-Mac approach . . . . . . . . . . . . . . . 17

5.1 Woods-Saxon parametrization of single-particle potentials . . . . . . . . . 18

5.2 Shell effects in $\alpha$-decay chains of $Z=120$ nuclei 21

5.3 Mass excesses and $Q_{\alpha}$-values . . . . . . . . 23

5.4 Quasiparticle levels in nuclei of $\alpha$-decay chain of ${ }^{295} 119$ and ${ }^{295,297} 120 \ldots . . \ldots 23$

5.5 Fusion-evaporation reactions for the production of SHN with $Z=119$ and $120 \ldots 26$

$5.5 .1 B_{f}-B_{n}$ in SHN . . . . . . . . 27

5.5.2 Fusion $Q$-values . . . . . . . . . . 28

5.5.3 Predictions of evaporation residue crosssections . . . . . . . . . 28

6 Isotopic trends of the nuclear surface properties of spherical nuclei . . . . . . . . . . . . . 30

6.1 EDF methods to nuclear ground-state density distributions . . . . . . . . . . . 31

6.1.1 Method I. GEDF . . . . . . . . . . . 31

6.1.2 Method II. Phenomenological Fayans EDF 31

6.2 Nucleon-density distribution . . . . . . . . . 33

6.3 Nucleus-nucleus interaction potential . . . . 36

7 Polarization of nuclear surface in deformed nuclei . 38

7.1 Parameters of nucleon-density distribution . . . 38 
7.2 Diffuseness polarization . . . . . . . . . . 39

7.3 Role of surface polarization effects in nuclear dynamics . . . . . . . . . . . 40

7.4 Correlation between observed $\alpha$-decays and changes in neutron or proton skins from parent to daughter nuclei . . . . . . . . . . . . . 41

8 EDF approach to predictions of the structure of SHN 42

8.1 Isotone chains of SHN . . . . . . . . . 42 8.1.1 $N=149$ isotone chain . . . . . . . . 43

8.1.2 $N=151$ isotone chain . . . . . . . 44

8.2 Odd-Odd SHN . . . . . . . . . . . . . 44

9 EDF approach to $\beta$-decay and EC . . . . . . . . 47

9.1 Heavy actinides and SHN . . . . . . . . . . . 47

$9.2 \beta$-decay half-lives and $\beta$-delayed multi-neutron emission in heavy nuclei . . . . . . . . . . . 49

9.3 EC cross-sections for neutron-rich $N=50$ nuclei in massive stars . . . . . . . . . . . . 50

10 Fission and cluster radioactivity in actinides and SHN 50

10.1 Fission barriers . . . . . . . . . 5 50

10.2 Spontaneous fission modes and lifetimes . . . . 53

10.3 Cluster radioactivity . . . . . . . . . . . . 53

11 Summary . . . . . . . . . . . . . . 5 55

References . . . . . . . . . . . . . 56

\section{Introduction}

The experiments on complete fusion reactions with a ${ }^{48} \mathrm{Ca}$ beam and various actinide targets were successfully carried out at FLNR (Dubna), GSI (Darmstadt), and LBNL (Berkeley) [1-21] in order to synthesize superheavy nuclei (SHN) with $Z=112-118$. The SHN with $Z=112$ and 113 were also synthesized at GSI (Darmstadt) and RIKEN (Tokyo) in the cold fusion reactions with target-nuclei ${ }^{208} \mathrm{~Pb}$ and ${ }^{209} \mathrm{Bi}[12,22-25]$. Production cross-sections and structural properties of SHN are governed by the competition of bulk properties and microscopic dynamics. The mass and charge dependence of binding energies as well as fusion and fission cross-sections are reasonably well described by the collective macroscopic degrees of freedom as in the liquid-drop model. In detail, however, the quantal nature of nuclei is found to be of utmost importance. Shell effects play a central role for the production, stability, and spectroscopy of SHN as has been well known since the early days of SHN research. Hence, the single-particle dynamics is the driving force for the abundance pattern of massive nuclei. In other words, the nuclear mean field plays a dominant role in understanding the properties of very heavy nuclei by controlling the overall binding and providing simultaneously a stabilization mechanism through the appearance of shell closures. Nuclear mass and reaction models must account for both the classical macroscopic and the quantal microscopic aspects of the nuclear dynamics; see, e.g. Ref. [26].
Probably the best-known macroscopic mass model is the Bethe-Weizsäcker mass formula $[27,28]$ expressing nuclear masses as a function of proton and neutron numbers. This scheme is still in use, known since then as the nuclear liquiddrop model. With a surprisingly small number of parameters such mass formulas are able to describe nuclear masses with impressing accuracy over essentially the whole nuclear chart. The liquid-drop model emphasizes the macroscopic degrees of freedom by integrating out the microscopic structure of nuclei. A partial re-implementation of microscopic aspects is attempted by the microscopic-macroscopic (MicMac) method. In a hybrid approach, microscopic shell corrections, taken from outside sources, are added in an ad hoc manner. Erstwhile, this was accomplished by the Strutinsky method [29,30], but afterwards the methods were significantly extended and refined; see, e.g., Refs. [31-52]. As a purely phenomenological recipe, the single-particle shell structure, obtained with a static potential, is used and has been found appropriate for providing the required microscopic corrections [22,44,45,53-65].

Typically, the Mic-Mac models use phenomenological single-particle potentials of Woods-Saxon (WS) type, supplemented for protons by the Coulomb potential in uniformly charged drop approximation. The WS parameters are adjusted to known nucleon separation energies where the radius is assumed to evolve as $A^{1 / 3}$ and the diffusivity parameter is kept fixed. The potential depth, left as the only free parameter, is adjusted to data. The Mic-Mac methods have their undisputable merits. Because of their simple structure they are perfectly well suited for large scale exploratory studies over essentially the whole $(Z, N)$ plane. As such, they are still indispensable tools for heavy and superheavy nuclei research, including not only bulk properties but also dynamical processes like fusion and fission reactions. The nuclear ground-state masses and deformations, actinide fission-barrier heights, spontaneous fission half-lives, fission-fragment charge yields, electron capture (EC) delayed fission and some prompt neutron-capture data were successfully described within the Mic-Mac approach [44,45,53-65].

From the theoretical point of view, there is no immediate connection of such a phenomenological potential model with the mass formula of the macroscopic part of the approach. Thus, extrapolations into unknown mass regions are very likely to suffer from uncontrollable inconsistencies. This problem is seen, for example, in the discrepancies in the predicted proton shell closures. The Mic-Mac models in their traditional formulation have been predicting shell closures for $Z=114$ and $N=184$. The fully microscopic theories based on energy-density functionals (EDF) mostly lead to the shell closures in the region $Z=120-126$ and $N=184$. A promising result was obtained in Refs. [66-73] pointing to a strategy for possible further improvements: By slight param- 
eter adjustments of the WS or Nilsson potentials, the proton shell closure in the Mic-Mac approach could be shifted indeed from $Z=114$ to $Z=120$. In view of many positive aspects, it is worthwhile trying to put the Mic-Mac method on firmer foundations. An important step in this direction is to improve the microscopic content. The Mic-Mac method may gain predictive power by an improved treatment of meanfield dynamics beyond the simple single-particle potential models. Within the framework of the density functional theory (DFT), the self-consistent variational methods provide the proper approaches for the derivation of single-particle potential and residual interactions.

In this review, we discuss the status of incorporating into the Mic-Mac approach elements of self-consistent meanfield descriptions by taking advantage of well established EDFs. Nuclear EDFs construct a mass formula on microscopic grounds by solving iteratively a self-consistency cycle for single-particle potentials and wave functions where convergence is controlled by their re-insertion into the EDF. These calculations lead to the self-consistent Hartree-FockBogoliubov (HFB) mean-field potentials which, of course, could be used as they are. However, for practical purposes, especially encountered in fusion and fission studies, the Schrödinger-equivalent mean-field potentials of an easy to handle structure are required. Therefore, in the second step we discuss in detail the general scheme for the derivation of the Schrödinger-equivalent single-particle potentials from the microscopic results of either non-relativistic or covariant EDFs. For numerical applications, parametrizations of the potentials in terms of WS form factors are derived which are most appropriate for the use in the Mic-Mac method. Moreover, the WS-representation gives insight into the mass and charge dependence of the proton and neutron potentials which otherwise is hidden in the self-consistent mean fields.

In Sect. 2, a brief overview of nuclear EDF is given as far as this is relevant for the purpose of this review. In Sect. 3, we sketch by the example of the Giessen EDF (GEDF), the methods used to derive a (non-relativistic) EDF in an ab initio manner from free-space NN interactions by Brueckner-HartreeFock (BHF) theory where the Brueckner $G$-matrix plays a central role. The overall achievements of such an EDF are discussed for infinite symmetric and asymmetric nuclear matter, including pairing and connecting to the Landau-Migdal (LM) theory.

In Sects. 4 and 5, we address a central issue of this review, namely the question how to connect microscopic selfconsistent methods to heuristic approaches like the Mic-Mac model, based on phenomenological mean-field potentials. The problem behind that question is that large scale studies on the structure and production of SHN rely on the availability of flexible theoretical methods, allowing to explore the parameter space in detail in finite time and with acceptable numerical effort. The approaches based on purely phenomenological input, come to their limits, once the region of known elements is left. Fully self-consistent microscopic approaches, on the other hand, require still too much resources, inhibiting their systematic use. Hence, a hybrid approach is on demand, incorporating the predictive power of microscopic methods and the flexibility of the heuristic description. A deconvolution method is introduced in Sect. 4 allowing to reconstruct the proper EDF by inversion from otherwise given nucleonic self-energies. In Sect. 5, we apply the WS potential extracted from the self-consistent approaches to calculate the shell effects and quasiparticle levels in $\alpha$-decay chains of SHN with $Z=119$ and 120 , the mass excesses and $Q_{\alpha}$-values for SHN with $Z=110-120$, and the evaporation residue cross-sections for nuclei with $Z=119$ and 120 .

The isotopic trends of the nuclear surface properties are presented in Sect. 6. The influence of the deformation on nuclear diffuseness is discussed in Sect. 7. The role of surface polarization effects in nuclear dynamics, and the correlation between observed $\alpha$-decays and changes in neutron (proton) skins from parent to daughter nuclei are also analyzed. The description and prediction of quasiparticle structures in the heaviest nuclei with the non-relativistic EDF models are presented in Sect. 8. The role of $\beta$-decay and EC in heavy and SHN is discussed in Sect. 9. In Sect. 10, we show advances in the study of spontaneous fission and cluster radioactivity within the framework of relativistic and non-relativistic EDF models.

\section{Overview of self-consistent nuclear-structure methods}

DFT and related EDF schemes are the only available approaches of being capable to describe microscopically nuclear matter and finite nuclei over essentially the whole mass table. Since the seminal work of Kohn, Sham, and Hohenberg [74-76], DFT has become an important and universal theoretical tool for quantum many-body systems. The merits of DFT also for nuclear theory became clear in the early 1970s when Vautherin and Brink [77,78] showed the wealth of the Skyrme energy-density functional (SkEDF) in conjunction with Hartree-Fock (HF) theory for nuclear research. The general structure of nuclear EDF is

$$
\begin{aligned}
\mathcal{E}\left[\psi^{\dagger}, \psi\right]= & \mathcal{T}\left[|\nabla \psi|^{2}\right]+\frac{1}{2} \mathcal{E}_{S}\left[\psi^{\dagger}, \psi\right]+\frac{1}{2} \mathcal{E}_{P}\left[\psi^{\dagger}, \psi\right] \\
& +\frac{1}{2} \mathcal{E}_{C}\left[\psi^{\dagger}, \psi\right]
\end{aligned}
$$

including, in the order of appearance, the kinetic-energy density, particle-hole-type strong interaction density, the particle-particle-type pairing energy density, and the Coulomb interaction energy density. The energy density is a functional of the nucleon field operators $\psi=\left\{\psi_{s Z}, \psi_{s N}\right\}$ for protons $(q=Z)$ and neutrons $(q=N)$ with spin projec- 
tions $s= \pm 1 / 2$. As usual, they may be expressed in terms of an appropriate basis of single-particle wave functions $\phi_{q \alpha}(\mathbf{r})$, where $\mathbf{r}$ and $\alpha=\{n, \ell, j, m\}$ denote the space coordinate and the principal quantum number, the orbital angular momentum, the total angular momentum, and the magnetic quantum number, respectively, connected with the corresponding creation and annihilation operators [79]. By symmetry reasons and conservation laws, the EDF is in fact a functional of bilinear forms of the field operators. Important quantities, establishing the contact to a given physical system and its thermodynamics, are the number densities $\rho=\left\{\rho_{Z}, \rho_{N}\right\}$, the kinetic densities $\tau=\left\{\tau_{Z}, \tau_{N}\right\}$, and nucleonic current densities $\iota=\left\{\iota_{Z}, \iota_{N}\right\}$ appearing in the spin-orbit parts, in all cases composed of proton and neutron components. The Coulomb energy is determined by the charge density obtained by folding $\rho_{Z}$ and $\rho_{N}$ with the respective nucleonic charge form factors. The pairing EDF is given by the anomalous density $\kappa=\left\{\kappa_{Z}, \kappa_{N}\right\}$. The explicit expressions of the densities and currents depend on whether the non-relativistic or the covariant formalism is used.

The SkEDF is a purely phenomenological parametrization of the non-relativistic Hamiltonian density of a nuclear system, expressed in terms of densities, kinetic densities, and spin-currents of protons and neutrons. Its structure corresponds roughly to an approximation of a nuclear EDF up to quadratic terms in momenta. Central spin and isospindependent terms and the spin-orbit term corresponding to momentum-dependent contact interactions up to order $p^{2}$ are included. Soon after the SkEDF, the Gogny EDF [80] was introduced, incorporating finite-range interactions. Over the years, a multitude of SkEDFs has been derived with slight variations in the parametrizations. Typically, about ten parameters are used which have to be fitted to the data. Widely used versions of the non-relativistic SkEDF are found, e.g., in Refs. [81,82], The SkHFB approach is equally well suitable for spherical and deformed nuclei. An argument in favor of the SkEDF approach is the use in dynamical surveys in the time-dependent Hartree-Fock (TDHF) or Hartree-FockBogoliubov (TDHFB) theory. These methods have been applied already in the 1970s [83]. Although lacking dissipative interactions, they still belong to the standard approaches for theoretical studies of nuclear fusion [84-87] and fission $[88,89]$ reactions.

However, as a caveat one notes that even for the same set of operators, the parameter values depend on the selection of experimental input data. There are correlations between different parameters hinting at the necessity to improve the functional structure of the EDF [90,91]. The fit only to binding energy provides already a satisfactory description of nuclear properties [92]. With those parameters the uncertainties in the description of nuclear radii (and surface thicknesses) remain on an acceptable level, confirming a strong correlation of the nuclear radius with binding energy. Extrapolations to exotic nuclei show, of course, increasing uncertainties when moving away from the mass and charge regions considered in the fit. These shortcomings in mind, in recent years a large scale research project was started in the US by the Unified Nuclear Energy Density Functional (UNEDF) collaboration. An upto-date version is the functional UNEDF2 [93,94]. The difference between the theoretical predictions obtained by that functional and the experimental data for the two-neutron $S_{2 n}$ and the two-proton $S_{2 p}$ separation energies remained within $2 \mathrm{MeV}$ for heavy nuclei even after the inclusion of additional experimental constraints. This also led to the conclusion that the standard Skyrme functional could not be further refined and that new forms of functionals have to be formulated. Indeed, these functionals being good for the description of the nuclear properties cannot reproduce well the nucleusnucleus interaction. For example, the fusion threshold energy obtained with different Skyrme interactions varies within 20 $\mathrm{MeV}$ for the ${ }^{16} \mathrm{O}+{ }^{16} \mathrm{O}$ reaction [95]. In order to improve the predictive power of the SkEDF, the set of operators may be enlarged. The finite-range interactions have been improved by adding the tensor term [96,97]. This important addition considerably extended the set of the EDF used [98-109]. However, this scheme is found to be only partially successful. For a recent discussion on the still controversial extension to non-central interactions by including higher rank tensor operators we refer to [110].

Soon after the advent of the Skyrme model, attempts started to derive a nuclear EDF on microscopic grounds. Negele and Vautherin were among the first to show the path to such an $a b$ initio EDF. Their influential approach [111] started from the BHF theory and then using the density matrix expansion (DME) method to reduce the highly non-local BHF $G$-matrix interactions to a local form as in the Skyrme model. The same spirit is underlying the nonrelativistic GEDF in starting from the Brueckner $G$-matrix interactions [112] but supplementing later three-body forces [113,114]. The new aspect of GEDF method was to express interactions in terms of meson exchange interactions with density-dependent coupling constants [112]. Such a formulation has the clear conceptual advantage that the EDF becomes a functional of bilinears of the nucleon field operators.

In parallel, a covariant version of the GEDF was derived, using at the time of publication for the first time the DiracBrueckner $G$-matrix interactions for the covariant EDF (CEDF) [115-118]. The essence of the density-dependent relativistic hadron (DDRH) field theory is again to recast the Dirac-Brueckner-Hartree-Fock (DBHF) interactions into the Hartree-like Lagrangian with Lorentz-invariant densitydependent meson-baryon vertex functionals, but retaining all relevant intrinsic and relativistic symmetries. In widespread applications to asymmetric nuclear matter and nuclei, neutron stars, and hypernuclei [119-121] the success of the DDRH approach was demonstrated. Somewhat later, the 
DDRH scheme was reconsidered on a purely phenomenological level by Typel and Wolter [122] and on a much larger scale also Ring and collaborators [123]. The merits (the natural description of the spin, pseudospin, and timeodd potential) and achievements of CEDF and relativistic mean-field (RMF) theories including the versions with either finite-range meson fields or zero-range point-coupling interactions were reviewed recently in Ref. [124]. The status of work on the derivation of $a b$ initio CEDF along the line of the early DDRH theory and beyond by making contact to chiral-symmetric interactions and effective field theory was also updated [124].

By now, numerous versions of non-relativistic Skyrme and Gogny-type EDFs [125] as well as CEDFs in Lagrangian formulation [123,124,126-143] are available and used for systematic nuclear dynamics and structure investigations over the entire periodic table. The multidimensionallyconstrained CEDF and CEDF on 3D lattices have recently been introduced to describe nuclear reactions (i.e., fission) and strongly deformed exotic states [138-143]. The EDFplus-HFB approaches describe quite successfully nuclear binding energies, nuclear shapes and sizes of beta-stable and beta-unstable nuclei; see e.g., [77, 78, 112, 115, 125, 127, 129, 136-138,144-161]. Thus, the Mic-Mac approaches have reached a high accuracy in reproducing nuclear masses, at least in the known mass region. For extrapolations into the unknown parts of the mass table they are still notoriously suffering from diverging predictions. Recent analyses, as in [162], indicated that the phenomenological Mic-Mac approaches seem to have reached a limit, making unlikely further improvements on the accuracy of data reproduction. The reason could be related to the ad hoc, albeit physically motivated, choice of the operator structure lacking a clear strategy for systematic extensions. Microscopically constructed EDFs based on the Brueckner or Dirac-Brueckner $G$-matrix interactions as in $[112,115,162-165]$ or using the order-byorder methods of chiral effective field theory $(\chi \mathrm{EFT})$ [166] are of a well-defined diagrammatic structure, allowing systematic improvements.

In the relativistic theory, the central and spin-orbit singleparticle potentials are determined in a unified manner by the same set of scalar and vector fields. In contrast, these potentials are formally independent in the non-relativistic theory. So, the relativistic theory provides a more consistent description of the spin-orbit coupling and useful guidance for the non-relativistic EDF $[129,135]$. For example, the spin-orbit properties in the CEDF were used in Ref. [167] to improve the isospin dependence of the spin-orbit potential in the nonrelativistic Skyrme-type functionals. In Ref. [168], the nonrelativistic reduction of CEDF theory was realized as in the similarity renormalization group method. This allows us to bridge the relativistic and non-relativistic EDF theories and to deepen our understanding on the interplay between the central and spin-orbit potentials for the Mic-Mac approach [169] and between the scalar and vector fields [168].

A quite general point of view is taken in the theory of the finite Fermi systems (TFFS) going back to Landau and worked out in much detail by Migdal [170-172]. The approach is based on the many-body Green function theory and takes advantage of Landau's renormalization procedure of interactions [170]. Both phenomenological and microscopic methods are in use [146,173-176]. The LM theory provides methods for deriving from a given EDF renormalized residual interactions and effective single-particle operators both in the particle-hole and the particle-particle channels, respectively. On the formal level the LM-approach corresponds to an expansion of a given nuclear EDF around a reference state into a power series of one-body spin-isospin density and current matrices. The theory leads to effective density-dependent quasiparticle interactions, acting as restoring forces in the abstract space of density matrices. The central quantities are the LM parameters. These dimensionless coefficients characterize the strength, momentum dependence, and other properties of residual interactions in the various spin-isospin channels. The LM parameters are universal quantities, depending mainly on the density of the system. Moreover, these parameters are directly related to the important thermodynamical properties of nuclear matter, among others the compressibility, symmetry energy, and velocity of sound. A covariant formulation of LM theory was worked out by Baym and Chin [172].

In Refs. [146,147], the local EDF was developed whose main contributions are the volume part for the description of homogeneous matter and the surface part to treat finite nuclei. In Refs. [148,149], the Barcelona-CataniaParis-Madrid functional was proposed using the calculations for nuclear matter. The phenomenological functional SeaLL1 [150] with only seven adjustable parameters, four of them based on the generalization of the liquid-drop model and the remaining three are constrained by the shell effects, pairing correlations, and the slope of the symmetry energy, describes reasonably well single-particle spectra, charge radii and twonucleon separation energies of even-even nuclei with $A \geq$ 16. In Refs. $[158,159]$, a new method was suggested to construct the EDF for nuclei from a given immutable equation of state (EoS). The method takes advantage of a natural ansatz for homogeneous nuclear matter, the Kohn-Sham framework, and the Skyrme formalism. The EDF is systematically expanded in powers of the Fermi momentum or cubic root of the density. The binding energies and the sizes of the closed(sub)shell nuclei were successfully described $[158,159]$. A simple and manageable method [177] was developed for deriving coupling constants of model EDF directly from $a b$ initio calculations performed for finite fermion systems. This method does not rely on properties of infinite fermion systems but on the $a b$ initio calculations in finite systems. 


\section{The Giessen energy-density functional}

\subsection{Energy-density functionals and microscopic interactions}

The non-relativistic GEDF and the covariant DDRH approach as well as more recent formulation along those lines are defined by BHF or DBHF $G$-matrix interactions, respectively. The common central aspect of microscopically derived EDF models is to express the medium-dependence of interactions in terms of screened interaction vertices, depending on the density of the system. In this section, the non-relativistic GEDF approach is reviewed as an example for connecting inmedium interactions and DFT. The $G$-matrices are expanded into a superposition of one-boson exchange (OBE) nucleonnucleon (NN) interactions with density-dependent mesonnucleon vertex functionals [112]. For that aim, we use the afore mentioned DME reduction scheme [111,178]. Interactions are used in antisymmetrized form, i.e., accounting for the Pauli principle by attaching the NN-exchange operator $P_{12}$ to the interaction rather than to the matrix elements. Thus, we use $V_{N N}=\left(1-P_{12}\right) U_{N N}$. The evaluation of $V_{N N}$ is especially simple in infinite matter and momentum representation $[112,114,179]$. However, it is well known that descriptions in terms of only two-body $G$-matrix interactions neither reproduce properly the empirically known EoS of infinite nuclear matter nor do they lead to proper results for finite nuclei. Irrespective of the choice of two-body interactions, the results are falling in the Coester-band, uncomfortably far off the data. In order to cure this problem, the threebody forces as in the Urbana approach [180] were added to the GEDF interactions. In order to retain the EDF structure as simple as possible, the three-body effects were included into the $\mathrm{NN}$ vertex functionals as an additional density-dependent contribution. This required to readjust slightly the two-body parameters in order to describe the self-energies and the EoS of symmetric nuclear and pure neutron matter. An advantage of the GEDF OBE-approach is the transparent operator structure. For example, the energy densities of the rank-0 central interactions are given by the renormalized spin-isospin $G$ matrix interactions

$$
\mathcal{G}(1,2 \mid \rho)=\sum_{S, T=0,1} G_{S T}(1,2 \mid \rho)\left[\boldsymbol{\sigma}_{1} \cdot \boldsymbol{\sigma}_{2}\right]^{S}\left[\boldsymbol{\tau}_{1} \cdot \boldsymbol{\tau}_{2}\right]^{T},
$$

where spin and isospin are denoted by the Pauli matrices $\sigma_{1,2}$ and $\boldsymbol{\tau}_{1,2}$, respectively. In momentum space, we have

$G_{S T}\left(\mathbf{k}_{1}, \mathbf{k}_{2} \mid \rho\right)=\sum_{m} V_{m}^{S T}(\rho) Y_{m}\left(\left|\mathbf{k}_{1}-\mathbf{k}_{2}\right|\right)$,

with the Yukawa meson-propagators $Y_{m}(p)=m^{2} /\left(m^{2}+p^{2}\right)$ and density-dependent vertex functionals accounting for medium-dependent screening effects, antisymmetrization, and three-body renormalization.
In infinite matter, the (antisymmetrized) interaction energy densities are given by

$$
\begin{aligned}
\mathcal{E}_{S T}(\rho)= & \frac{1}{4} \operatorname{tr}_{s q} \operatorname{tr}_{s^{\prime} q^{\prime}} \int \frac{d^{3} k_{1}}{(2 \pi)^{3}} \int \frac{d^{3} k_{2}}{(2 \pi)^{3}} G_{S T}\left(\mathbf{k}_{1}, \mathbf{k}_{2} \mid \rho\right) \\
& \times n_{s q}^{S T} n_{s^{\prime} q^{\prime}}^{S T} \Theta\left(k_{F_{q}}-k_{1}\right) \Theta\left(k_{F_{q^{\prime}}}-k_{2}\right),
\end{aligned}
$$

for nucleons of spin $s$ and isospin (respective charge) $q$. The spin-isospin occupation numbers are defined as

$n_{s q}^{S T}=\left\langle s q\left\|\boldsymbol{\sigma}^{S} \boldsymbol{\tau}^{T}\right\| s q\right\rangle$.

The Fermi momenta $k_{F_{q}}=\left(3 \pi^{2} \rho_{q}\right)^{1 / 3}$ are given by the proton and neutron ground-density distributions $\rho_{p, n}=$ $\operatorname{tr}_{s}\left\langle A\left|\psi_{s Z, s N}^{\dagger} \psi_{s Z, s N}\right| A\right\rangle$, the latter being functionals of the field operators. In infinite matter, the particle densities are expressed by the isotopic ratio $\xi \sim Z / A$ and the total density $\rho$ as $\rho_{p}=\xi \rho$ and $\rho_{n}=(1-\xi) \rho$.

In spin-saturated nuclear matter, only the elements $\mathcal{E}_{00}$ and $\mathcal{E}_{01}$ lead to non-vanishing expectation values. In isospinsaturated symmetric nuclear matter $\mathcal{E}_{01}$ does not contribute to the energy. The first variation leads to the momentumdependent isoscalar and isovector mean-field self-energies,

$$
\begin{aligned}
& \Sigma_{0}(k, \rho)=U_{00}(k, \rho)+U^{(r)}(k, \rho), \\
& \Sigma_{1}(k, \rho)=U_{01}(k, \rho),
\end{aligned}
$$

with the isoscalar $U_{00}$ and isovector $U_{01}$ mean-field potentials. Here, $\rho=\rho_{p}+\rho_{n}$ is the isoscalar nuclear density. The rearrangement potential $U^{(r)}$ results from the variational derivative of the in-medium interaction vertices. In non-relativistic and covariant EDF theory, $U^{(r)}$ is an indispensable part of the mean-field self-energy because it ensures thermodynamical consistency of the theory by fulfillment of the Hugenholtz-van Hove theorem [112,116]. In order to maintain the isospin-symmetry of the EDF in accordance with the underlying elementary $\mathrm{NN}$ interactions, the vertices must be invariant under isospin transformations. This is given if the vertices are isoscalar quantities, implying that they are functionals of only $\rho$. Consequently, only $\Sigma_{0}$ is affected by rearrangement contributions.

The proton and neutron self-energies are obtained:

$$
\begin{gathered}
\Sigma_{p}(k, \rho)=\Sigma_{0}(k, \rho)-\Sigma_{1}(k, \rho), \\
\Sigma_{n}(k, \rho)=\Sigma_{0}(k, \rho)+\Sigma_{1}(k, \rho) .
\end{gathered}
$$

In symmetric nuclear matter, $\Sigma_{1}$ vanishes and protons and neutrons experience the same mean fields-except in finite nuclei for the Coulomb interactions. Since in the mass and momentum regions of interest $\Sigma_{1}$ is positive, in neutronrich matter more attractive proton potentials are encountered while neutrons move in more shallow potentials, as reflected in the evolution of separation energies with neutron excess. 


\subsection{Single-quasiparticle equations of motion}

For practical applications, the EDF is evaluated for a specific system $|A\rangle$, defined by the content of $Z$ protons and $N$ neutrons with $A=N+Z$. The single-particle and residual interactions in this system are then obtained by a second order expansion in the abstract space of density matrices around a reference state $|A\rangle$ - typically the ground state of the system — with $E_{A}=\langle A|\mathcal{E}| A\rangle$,

$$
\begin{aligned}
\mathcal{E}(\rho) & \approx E_{A}+\sum_{q}\left(\left[T_{q}+\Sigma_{q}(\rho)\right] \delta \rho_{q}+\Delta_{q}(\rho) \delta \kappa_{q}\right) \\
& +\sum_{q, q^{\prime}} f_{q q^{\prime}}(\rho) \delta \rho_{q} \delta \rho_{q^{\prime}}+\sum_{q, q^{\prime}} r_{q q^{\prime}}(\rho) \delta \kappa_{q} \delta \kappa_{q^{\prime}},
\end{aligned}
$$

plus spin-dependent and electromagnetic contributions which are not shown here. The interactions are functions of $\rho$. The energy $E_{A}$ is self-consistently evaluated in an iterative cycle up to the required accuracy. The density variations around the reference state are given in terms of the nucleon field operators $\psi_{s q}^{\dagger}$ and $\psi_{s q}$, which are suitably expanded into the basis of (Kohn-Sham) single-particle wave functions $\phi_{q \alpha}$ and the respective creation $a_{q \alpha}^{\dagger}$ and annihilation $a_{q \alpha}$ operators belonging to the states in $|A\rangle$ [79]. The number density and the pairing (or anomalous) density are given by

$$
\begin{aligned}
\delta \rho_{q} & =\operatorname{tr}_{s}\left\langle A\left|\psi_{s q}^{\dagger} \psi_{s q}\right| A\right\rangle=\operatorname{tr}_{s} \sum_{\alpha, \alpha^{\prime}} \phi_{q \alpha}^{*} \phi_{q \alpha^{\prime}}\left\langle A\left|a_{q \alpha}^{\dagger} a_{q \alpha^{\prime}}\right| A\right\rangle \\
\delta \kappa_{q} & =\frac{1}{2} \operatorname{tr}_{s}\langle A| \psi_{s q}^{\dagger} \psi_{s q}^{\dagger}+\text { h.c. }|A\rangle \\
& =\frac{1}{2} \operatorname{tr}_{s} \sum_{\alpha, \alpha^{\prime}}\left[\phi_{q \alpha}^{*} \phi_{q \alpha^{\prime}}^{*}\left\langle A\left|a_{q \alpha}^{\dagger} a_{q \alpha^{\prime}}^{\dagger}\right| A\right\rangle+\text { h.c. }\right] .
\end{aligned}
$$

Hence, a quadratic form in $\delta \rho_{q}$ and $\delta \kappa_{q}$ is obtained, describing harmonic oscillations in the abstract space of density matrices. The first order terms define the dynamics of singlequasiparticle states, controlled by the kinetic-energy operator $T_{q}$, the potentials $U_{q}$, and the pairing field $\Delta_{q}$. The restoring forces described by $f_{q q^{\prime}}$ and $r_{q q^{\prime}}$ will be discussed below.

The particle equations of motion are derived by the first variation with respect to $\psi_{s q}^{\dagger}$ of the constrained EDF $\mathcal{E}^{\prime}=$ $\mathcal{E}\left[\psi^{\dagger}, \psi\right]-\lambda_{Z} Z-\lambda_{N} N$. The proton $Z$ and neutron $N$ number operators and the corresponding Lagrange multipliers, i.e., chemical potentials, $\lambda_{Z, N}$, act to conserve on average the corresponding proton and neutron numbers. Performing the Bogoliubov-Valatin rotation to quasiparticles, the result is the 2-by-2 set of coupled quasiparticle equations of motion

$$
\left(\begin{array}{cc}
T_{q}+U_{q}-e_{q \alpha+} & \Delta_{q} \\
-\Delta_{q}^{+} & T_{q}+U_{q}-e_{q \alpha-}
\end{array}\right)\left(\begin{array}{c}
u_{q \alpha} \\
v_{q \alpha}
\end{array}\right)=0,
$$

derived originally in different context by Gorkov [181] and introduced into the nuclear SkHFB theory by Dobaczewski et al. [182]. The GEDF results for dripline nuclei obtained by the full solution of the Gorkov equations are presented in Ref. [114]. The full set of quantum numbers (except isospin/charge), specifying the quasiparticle state, is contained in $\alpha$. The Hamiltonian matrix contains on the diagonal the mean-field Hamiltonian $T_{q}+U_{q}$ with the self-consistent potentials $U_{q}$. The quasiparticle state vectors are given by the particle-like $\left(u_{q \alpha}\right)$ and the hole-like $\left(v_{q \alpha}\right)$ components, respectively. These two components are coupled by the pairing field $\Delta_{q}$. As discussed in detail in [114], we solve the Gorkov equations by considering $s$-wave pairing, i.e. proton and neutron pairs of total spin $S=0$ and isospin $T=1$. For numerical calculations, the pairing interaction is chosen as the singlet-even Bonn potential, $V_{\text {pair }} \simeq V_{S E}$. The energies are expressed as $e_{q \alpha \pm}=\lambda_{q} \pm E_{q \alpha}$ with the quasiparticle energy $E_{q \alpha}=\left(e_{q \alpha+}-e_{q \alpha-}\right) / 2>\left|\Delta_{q}\right|$. In general, the particle-like and hole-like energies $e_{q \alpha \pm}$ do not coincide with the eigenvalues $\eta_{q \alpha}$ of the mean-field Hamiltonian.

The quasiparticle state vectors are normalized according to

$$
\begin{aligned}
& \int d^{3} r\left(u_{q \alpha}^{*}(\mathbf{r}) u_{q \beta}(\mathbf{r})+v_{q \alpha}^{*}(\mathbf{r}) v_{q \beta}(\mathbf{r})\right) \\
& =\delta_{\alpha \beta}\left\{\begin{array}{cc}
1 & , \lambda_{q}<e_{q \alpha}<2 \lambda_{q} \\
\frac{1}{4 \pi} \frac{1}{d\left(e_{q \alpha}\right)} \delta\left(e_{q \alpha}-e_{q \beta}\right), & e_{q \alpha}>2 \lambda_{q}
\end{array}\right\},
\end{aligned}
$$

where $d\left(e_{q \alpha}\right)$ is the density of states at energy $e_{q \alpha}$. The nucleon densities are given by

$$
\begin{aligned}
\rho_{q}(\mathbf{r})= & \int_{-\infty}^{+\infty} d e \sum_{\alpha}\left(\left|v_{q \alpha}(\mathbf{r})\right|^{2} \Theta\left(e-2 \lambda_{q}\right) \delta\left(e-e_{q \alpha}\right)\right. \\
& \left.+4 \pi d\left(e_{q \alpha}\right)\left|v_{q \alpha}(\mathbf{r})\right|^{2} \Theta\left(2 \lambda_{q}-e\right)\right)
\end{aligned}
$$

including the contributions from the discrete and the continuous part of the quasiparticle spectrum. Here, $\Theta$ is the step function. The anomalous density is

$$
\begin{aligned}
\kappa_{q}(\mathbf{r})= & \frac{1}{2} \int_{-\infty}^{+\infty} d e \sum_{\alpha}\left(v_{q \alpha}^{*}(\mathbf{r}) u_{q \alpha}(\mathbf{r}) \Theta\left(e-2 \lambda_{q}\right) \delta\left(e-e_{q \alpha}\right)\right. \\
& \left.+4 \pi d\left(e_{q \alpha}\right) v_{q \alpha}^{*}(\mathbf{r}) u_{q \alpha}(\mathbf{r}) \Theta\left(2 \lambda_{q}-e\right)\right)
\end{aligned}
$$

In a finite nucleus, the proton and neutron numbers are obtained by integration of the proton and neutron densities, respectively. Applications of the Gorkov approach to weakly bound halo nuclei and nuclei with a neutron skin are found in Ref. [114].

The BCS equations are recovered immediately in infinite nuclear matter: There, the state vector splits into a spin-space wave function and a reduced amplitude vector

$$
\left(\begin{array}{c}
u_{q \alpha}(\mathbf{r}) \\
v_{q \alpha}(\mathbf{r})
\end{array}\right) \mapsto \phi_{q \alpha}(\mathbf{r})\left(\begin{array}{c}
\bar{u}_{q \alpha}(\mathbf{k}) \\
\bar{v}_{q \alpha}(\mathbf{k})
\end{array}\right),
$$




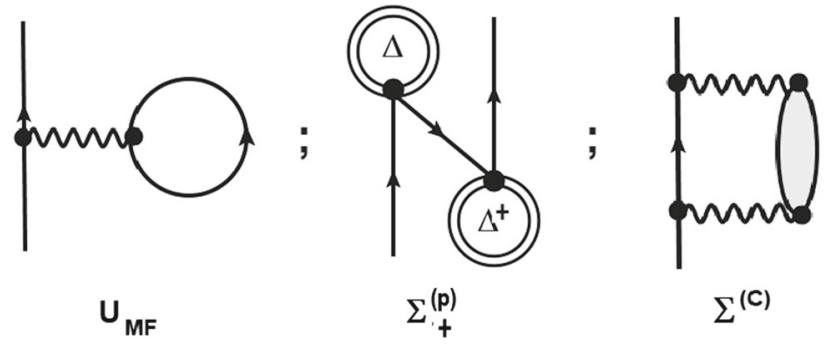

Fig. 1 Single-particle self-energies, considered in this work, include the effective (Hartree) mean-field potential (left), the pairing self-energy (center), and the core-polarization self-energy (right)

and the translational invariance of infinite matter demands that $\phi_{q \alpha}(\mathbf{r})$ is a plane wave carrying the quasiparticle momentum $\mathbf{k}$ times a Pauli spinor. In a finite nucleus, for $\phi_{q \alpha}(\mathbf{r})$ an eigenfunction with the proper quantum numbers of the mean-field Hamiltonian is used. In either case, $\phi_{q \alpha}(\mathbf{r})$ is integrated out and the conventional linear system of BCS equations for the reduced amplitudes and quasiparticle energies is obtained, but with the state-dependent gaps.

Last but not least, we point out that the channel coupling may be eliminated by the Feshbach projection in either of the two channels. This leads to formally homogeneous wave equations, but at the expense of state- and energy-dependent pairing self-energies which in the particle and hole channels are given by

$$
\begin{aligned}
& \Sigma_{q+}^{(P)}\left(e_{q \alpha+}\right)=\Delta_{q} g_{--}\left(e_{q \alpha-}\right) \Delta_{q}^{\dagger}, \\
& \Sigma_{q-}^{(P)}\left(e_{q \alpha-}\right)=\Delta_{q}^{\dagger} g_{++}\left(e_{q \alpha+}\right) \Delta_{q} .
\end{aligned}
$$

The propagation through the complementary channel is denoted by the Green function $g_{\text {干干. }}$. In Fig. 1, the (Hartree) mean field $U_{q}$, the pairing self-energy $\Sigma_{q \pm}^{(P)}$, and the dynamical core-polarization self-energy $\Sigma_{q}^{(C)}$, contributing another state-dependent self-energy [114], are diagrammatically displayed.

\subsection{GEDF results for infinite nuclear matter and}

Fermi-liquid parameters

Our results for the equations of state for symmetric and pure neutron matter are shown in Fig. 2. Interestingly, the bulk part of the density dependence is already taken into account by the two-body exchange terms while three-body interactions contribute in absolute terms only a minor part to the energy density. However, because of their different curvature these contributions are extremely important for a proper description of the EoS for both symmetric nuclear and pure neutron matter.

In the absence of residual interactions the ground state of nuclear matter is the Fermi gas of free quasiparticles remembering their elastic interactions by a modified energy-

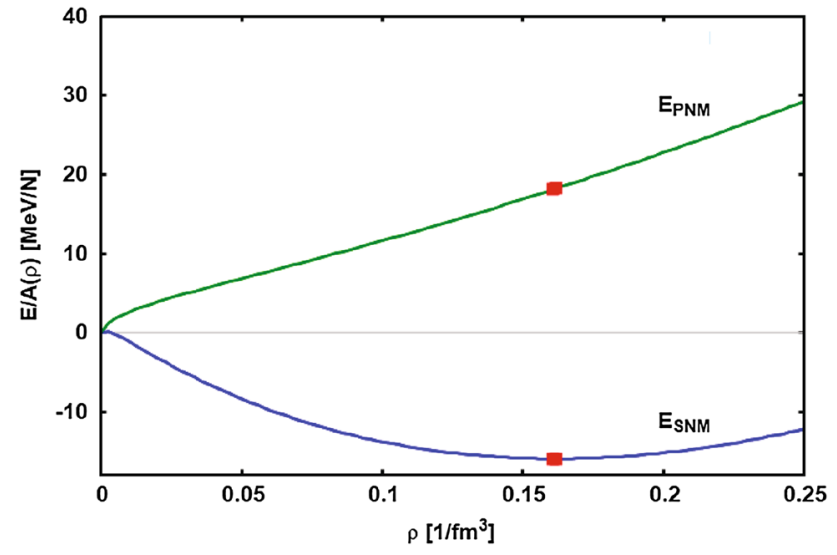

Fig. 2 The GEDF equation of state for symmetric $\left(E_{\mathrm{SNM}}\right)$ nuclear and pure neutron $\left(E_{\mathrm{PNM}}\right)$ matter. The values at saturation density $\rho_{\text {sat }}$ are marked by squares

momentum dispersion relation. However, the system may be driven about of equilibrium and react in a characteristic manner to perturbations. How that is happening, is contained in the last line of Eq. (10). The coefficients $f_{q q^{\prime}}$ are the restoring forces against perturbations of the normal densities, the coefficients $r_{q q^{\prime}}$ are the restoring forces against perturbations of the anomalous densities. The latter will not be considered further, because close to nothing is known about the related pair vibration modes.

The $f_{q q^{\prime}}$ are the two-quasiparticle scattering amplitudes at the Fermi surface, obtained in a self-consistent manner by the second variation of the EDF. As indicated in Fig. 3, in addition to the two-body scattering diagram one obtains two kinds of medium polarization diagrams that contribute, describing screening effects due to polarization of the background medium. As usual, we revert to an operator representation with $S=0,1$ and $T=0,1$ and to the dimensionless amplitude

$$
\mathcal{F}\left(\mathbf{k}, \mathbf{k}^{\prime} \mid \rho\right)=C\left(k_{F}\right) \sum_{S T} f_{S T}\left(\mathbf{k}, \mathbf{k}^{\prime} \mid \rho\right)\left[\boldsymbol{\sigma} \cdot \boldsymbol{\sigma}^{\prime}\right]^{S}\left[\boldsymbol{\tau} \cdot \boldsymbol{\tau}^{\prime}\right]^{T},
$$

normalized to the average density of states per unit energy at and per unit volume at the Fermi surface,

$C\left(k_{F}\right)=\frac{N_{s} M k_{F}}{2 \pi^{2} \hbar^{2}}$,

where $N_{s}=2$ is the spin multiplicity, $M$ is the average nucleon mass ${ }^{1}$ and $k_{F} \sim \rho^{1 / 3}$ is related to the total number density. At saturation density, one finds $C^{-1} \sim 300 \mathrm{MeV}$. $\mathrm{fm}^{3}$. By convention, the scattering amplitudes are repre-

\footnotetext{
${ }^{1}$ Note that some authors use instead of $M$ the average effective nucleon mass $M^{*}\left(k_{F}\right)$. Occasionally, also the isospin multiplicity $N_{t}=2$ is included.
} 


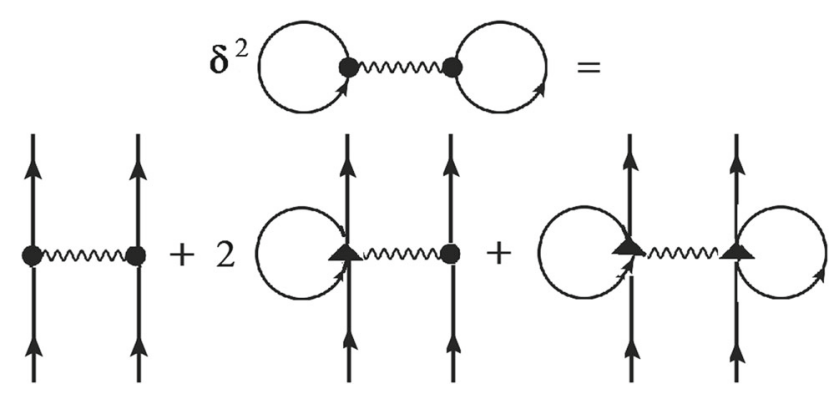

Fig. 3 Diagrammatic structure of residual interactions obtained by the second variation of the microscopic interaction energy density. The contributions depicted in the second line are from left to right: the two-body scattering term, in-medium vertex screening, and full rearrangement corrections due to static polarization of the medium

sented in terms of spin-isospin amplitudes. By partial wave expansion, we obtain

$$
\begin{aligned}
& \mathcal{F}\left(\mathbf{k}, \mathbf{k}^{\prime} \mid \rho\right)=\sum_{\ell} P_{\ell}\left(\cos \theta_{k k^{\prime}}\right) \\
& \quad \times\left(F_{\ell}(\rho)+F_{\ell}^{\prime}(\rho) \boldsymbol{\tau} \cdot \boldsymbol{\tau}^{\prime}+\left[G_{\ell}(\rho)+G_{\ell}^{\prime}(\rho) \boldsymbol{\tau} \cdot \boldsymbol{\tau}^{\prime}\right] \boldsymbol{\sigma} \cdot \boldsymbol{\sigma}^{\prime}\right),
\end{aligned}
$$

where only the central interactions of tensorial rank 0 are shown. Note that the LM amplitudes include by convention factors of $2 \ell+1$. Of special relevance are the amplitudes at the Fermi surface, $k=k^{\prime}=k_{F}(\rho)$, as also used here. The GEDF spin-isospin LM parameters are shown as functions of the density in Fig. 4. The rearrangement effects due to the static polarization of the Fermi sea induce considerable renormalization contributions which modify the leading two-body amplitude by screening effects. The screening is especially pronounced in the $S=T=0$ interaction channel.

Our results for $F_{S T}$, where $F_{00}=F_{0}, F_{01}=F_{0}^{\prime}$, $F_{10}=G_{0}$, and $F_{11}=G_{0}^{\prime}$, are shown in Fig. 4. At low densities, $F_{00}<-1$ is found which violates the stability condition $F_{\ell}>-(2 \ell+1)$. This condition is valid for all $\ell$ and all LM parameters. If violated, competing liquid and gas phases will occur. In fact, at low densities, nuclear matter may condense into a gas of nucleons and light clusters. Halo nuclei and neutron skins are indicative for the existence of such a regime and the effect has been studied in connection with ternary fission [183]. In the spin/isospin interaction channels the GEDF interactions are free of instabilities up to extreme values of the density. For a representative collection of SkEDF's, however, instabilities have been identified in the spin-isospin channel at densities beyond saturation [184]. For detailed discussions of the use of the LM interactions in investigations of excited states we refer to the literature; see, e.g., $[113,114]$ and further references therein.

The LM parameters are key quantities governing all sectors of nuclear dynamics, from the ground-state properties to the spectroscopy of nuclear matter and finite nuclei, thus

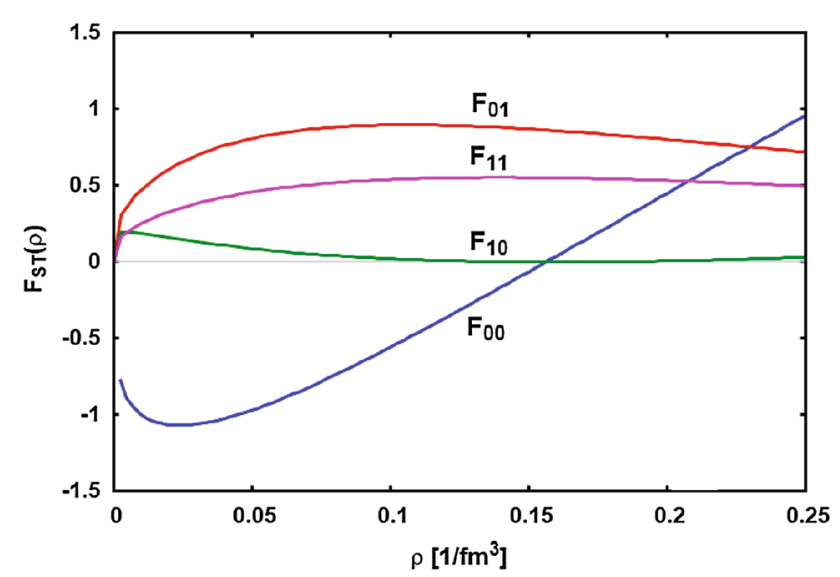

Fig. 4 GEDF Landau-Migdal parameters $F_{S T}$ in symmetric nuclear matter where $F_{00}=F_{0}, F_{01}=F_{0}^{\prime}, F_{10}=G_{0}$, and $F_{11}=G_{0}^{\prime}$

emphasizing again the importance of a consistent treatment of all sectors of the theory. Below, examples of their contributions to ground-state properties are given.

Important quantities for the thermodynamics of symmetric nuclear matter are the pressure density

$\mathcal{P}(\mathcal{E}(\rho))=\rho \frac{\partial \mathcal{E}(\rho)}{\partial \rho}$,

the pressure

$P(\rho)=\rho \frac{\partial}{\partial \rho} \frac{\mathcal{E}(\rho)}{\rho}$,

and the compression modulus

$$
\begin{aligned}
K_{\infty}(\rho) & =k_{F}^{2} \frac{\partial^{2}}{\partial k_{F}^{2}} \frac{\mathcal{E}(\rho)}{\rho} \\
& =\frac{3 k_{F}^{2}(\rho)}{M^{*}(\rho)}\left[1+F_{0}(\rho)\right],
\end{aligned}
$$

describing the curvature of the saturation curve. Here, $k_{F}(\rho)=\left(1.5 \pi^{2} \rho\right)^{\frac{1}{3}}$ is the local Fermi momentum. The effective mass,

$M^{*}(\rho)=M\left[1+\frac{1}{3} F_{1}(\rho)\right]$,

is determined by the spin- and isospin-scalar $p$-wave LM amplitude $F_{1}$ in (21). The speed of the first sound is related to the compression modulus via

$v_{s}^{2}=\frac{k_{F}^{2}}{3 M^{2}} \frac{1+F_{0}}{1+\frac{1}{3} F_{1}}=\frac{K_{\infty}}{9 M}$,

which is given in units of the speed of light in vacuum, $c$, and by causality $v_{s}<1$.

An important quantity characterizing isospin dynamics of nuclear systems is the symmetry energy, defined as the 
Table 1 Observables characterizing the equation of state of infinite symmetric nuclear matter. The results using non-relativistic [nr] (column 2, from Ref. [185]), and relativistic [re] (column 3, see text) kineticenergy densities are listed. Effective masses are given in unit of the free nucleon mass, $M_{N}$. In the last line, the speed of first sound is shown in units of the speed of light, $c$. All quantities are evaluated at the respective $\rho=\rho_{\text {sat }}$

\begin{tabular}{llll}
\hline Observable & Theory $[\mathrm{nr}]$ & Theory [re] & Empirical values and units \\
\hline$\rho_{\text {sat }}$ & 0.1617 & 0.1611 & $\sim 0.16\left[\mathrm{fm}^{-3}\right]$ \\
$E_{\text {sat }}$ & -15.91 & -16.01 & $\sim-16.0[\mathrm{MeV}]$ \\
$M^{*}$ & 0.71 & 0.71 & $\sim 0.6-0.8\left[\mathrm{M}_{N}\right]$ \\
$K_{\infty}$ & 247.05 & 262.89 & $210-260[\mathrm{MeV}]$ \\
$E_{\text {sym }}$ & 33.41 & 33.43 & $30 \pm 2[\mathrm{MeV}]$ \\
$L_{\text {sym }}$ & 53.86 & 50.88 & $43 \pm 16[\mathrm{MeV}]$ \\
$K_{\text {sym }}$ & -188.37 & -207.83 & $-30 \ldots-300[\mathrm{MeV}]$ \\
$v_{s}$ & 0.160 & 0.176 & {$[c]$}
\end{tabular}

second derivative in the isovector density $\rho_{1}=\rho_{n}-\rho_{p}$,

$E_{\text {sym }}(\rho)=\left.\frac{1}{2} \rho^{2} \frac{\partial^{2}}{\partial \rho_{1}^{2}} \frac{\mathcal{E}(\rho)}{\rho}\right|_{\rho_{1}=0}$,

which is directly proportional to the s-wave spin scalarisovector LM parameter

$E_{\text {sym }}=\frac{k_{F}^{2}}{3 M^{*}}\left[1+F_{0}^{\prime}\right]$.

The density dependence of $E_{s y m}$ is investigated by the Taylor-series expansion in $\delta \rho=\rho-\rho_{\text {sat }}$ :

$$
\begin{aligned}
E_{\text {sym }}(\rho) & \simeq E_{\text {sym }}\left(\rho_{\text {sat }}\right)+\delta \rho \frac{L_{\text {sym }}\left(\rho_{\text {sat }}\right)}{3 \rho_{\text {sat }}} \\
& +\delta \rho^{2} \frac{K_{\text {sym }}\left(\rho_{\text {sat }}\right)}{18 \rho_{\text {sat }}^{2}}+\cdots
\end{aligned}
$$

The slope parameter $L_{s y m}$ and symmetry compression modulus $K_{\text {sym }}$ are defined according to

$$
\begin{aligned}
& L_{\text {sym }}(\rho)=3 \rho \frac{\partial E_{\text {sym }}(\rho)}{\partial \rho}, \\
& K_{\text {sym }}(\rho)=9 \rho^{2} \frac{\partial^{2} E_{\text {sym }}(\rho)}{\partial \rho^{2}} .
\end{aligned}
$$

The relevant observables characterizing the GEDF equation of state of symmetric nuclear matter are listed in Table 1. The saturation point of nuclear matter, i.e., the minimum of the binding energy curve, appears at a density $\rho_{\text {sat }}$, where $P\left(\rho_{\text {sat }}=0\right)$. For comparison, also results for a version of GEDF using relativistic kinematics are shown, illustrating the minor influence of relativistic kinematics on the bulk properties of the EoS. $E_{\text {sym }}(\rho)$ is displayed as a function of the density in Fig. 5. The GEDF predictions for $L_{s y m}(\rho)$ and $K_{\text {sym }}(\rho)$ are shown in Fig. 6 . The underlying GEDF interactions together with the results of several RMF models were used in $[185,186]$ to study dipole modes of the nuclear skin and their influence on the reaction rates of stellar nucleosynthesis. The results are in a good agreement with earlier

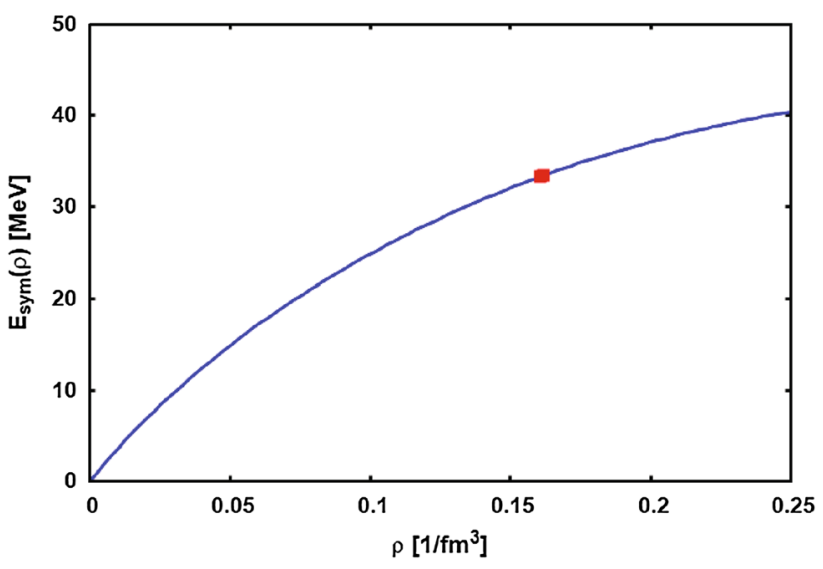

Fig. 5 GEDF symmetry energy in infinite nuclear matter as a function of nucleon density. The value at $\rho_{\text {sat }}$ is marked by a square

studies of symmetry energy effects in the neutron skin of finite nuclei [187].

In Table 1, also the empirical values are listed for various nuclear matter quantities. A vast amount of work is being devoted to extracting those values from various kinds of data. The major sources of information are heavy-ion collision, low-energy nuclear reactions, nuclear-structure data on neutron skins, dipole skin modes and giant resonance excitations, and, of course, nuclear mass formulas; see, e.g., [185-190]. Combining the results of various sources, we conclude that nuclear matter saturates at $\rho_{s a t}=0.16 \mathrm{fm}^{-3}$ with binding energy per nucleons of about $E_{B} \simeq-16 \mathrm{MeV}$. For the symmetry energy the value $E_{\text {sym }} \sim 30 \pm 2 \mathrm{MeV}$ is derived. $L_{\text {sym }}$ and $K_{\text {sym }}$ are less known. The value $L_{\text {sym }}=30 \pm 16$ $\mathrm{MeV}$ [185-188] is found and estimates mainly from heavyion collisions indicate the rather uncertain range of values $K_{\text {sym }}=-30 \ldots-300 \mathrm{MeV}$. Hence, much room is left for further investigations. 


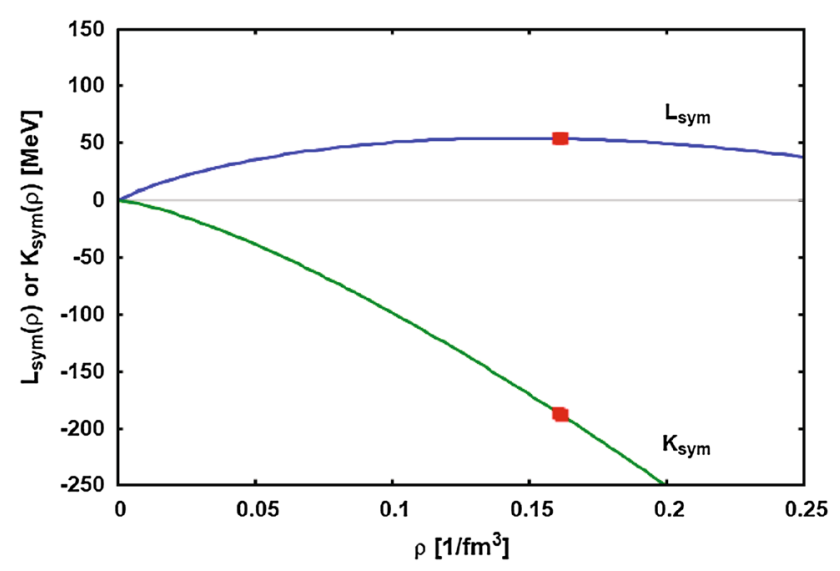

Fig. 6 GEDF results for the isospin pressure $L_{s y m}$ and the isospin compression modulus $K_{\text {sym }}$ as functions of the nucleon density. The values at $\rho=\rho_{\text {sat }}$ are marked by full squares

\subsection{Pairing in infinite nuclear matter}

For understanding the overall effect of pairing, it is instructive to examine first infinite nuclear matter. The pairing field (or gap function) is given by

$\Delta_{q}(\mathbf{k})=\int \frac{d^{3} k^{\prime}}{(2 \pi)^{3}} V_{p a i r}\left(\mathbf{k}, \mathbf{k}^{\prime}\right) \widetilde{\kappa}_{q}\left(\mathbf{k}^{\prime}\right)$,

with the anomalous or pairing density, given here as

$\widetilde{\kappa}_{q}(\mathbf{k})=\frac{\Delta_{q}(\mathbf{k})}{2 E_{q}\left(k, \Delta_{q}(\mathbf{k})\right)}$,

where $E_{q}\left(k, \Delta_{q}\right)=\sqrt{\left(\eta_{q}(k)-\lambda_{q}\right)^{2}+\Delta_{q}^{2}}$ is the quasiparticle energy with the mean-field single-particle energy $\eta_{q}(k)=\hbar^{2} k^{2} / 2 M_{q}^{*}+U_{q}(\rho)$ and momentum $k$. The solution of the nonlinear integral equation for pairing gap can be simplified by considering the different scales involved: The interaction varies on scales of the order of the pion mass, $m_{\pi} \sim 140 \mathrm{MeV}$, or larger. The pairing density, $\kappa_{q}$ is nonvanishing only in a narrow interval of width $\sim 2 \Delta_{q}\left(k_{F_{q}}\right)$, distributed symmetrically around the Fermi surface. These vastly different scales allow us to replace Eq. (33) with a very good approximation by

$$
\Delta_{q}(\mathbf{k}) \mapsto \Delta_{q}\left(k_{F_{q}}\right)=G_{\text {pair }}\left(k_{F_{q}}\right) \int \frac{d k}{2 \pi^{2}} k^{2} \widetilde{\kappa}_{q}(k) F(k),
$$

where the effective interaction functional,

$$
G_{\text {pair }}\left(k_{F_{q}}\right)=\left.\frac{1}{2} \int_{-1}^{+1} d \cos \theta_{k k^{\prime}} V_{\text {pair }}\left(\mathbf{k}, \mathbf{k}^{\prime}\right)\right|_{k=k^{\prime}=k_{F q}}
$$

is given by the $s$-wave component of $V_{\text {pair }}$. Since the pairing field has become independent of $\mathbf{k}$, the gap equation is

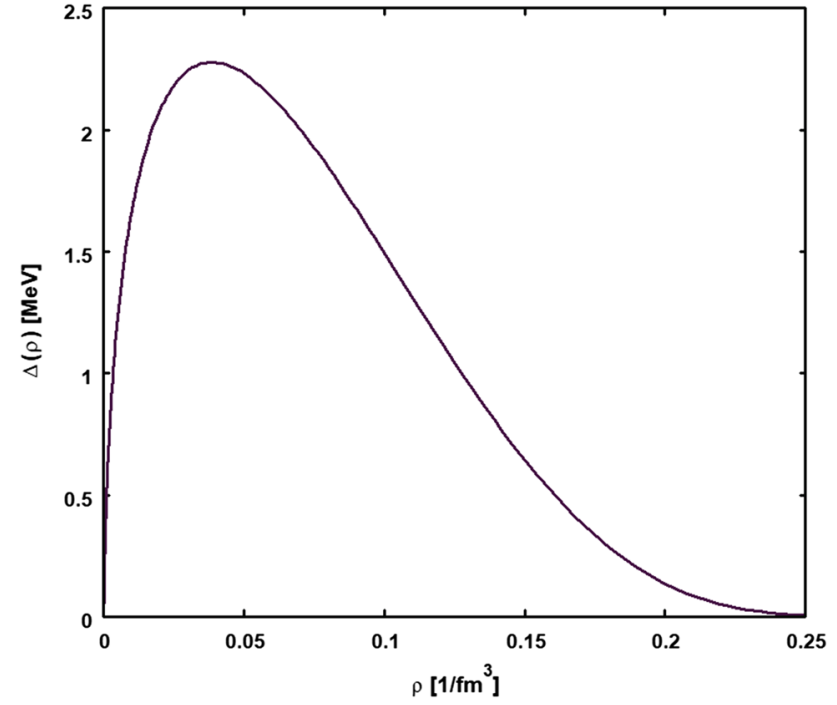

Fig. 7 Pairing gap in infinite symmetric nuclear matter at $k=k_{F}(\rho)$ as a function of density $\rho$. The pairing interaction is derived from the $s$-wave component of the (non-relativistic) singlet-even Bonn NNpotential

reduced to

$1-\left.G_{\text {pair }}\left(k_{F_{q}}\right) \int_{0}^{\infty} \frac{d k}{4 \pi^{2}} \frac{k^{2} F(k)}{E_{q}(k, \Delta)}\right|_{\Delta=\Delta_{q}}=0$.

The remaining integral is regularized by the cut-off form factor $F(k)$, a convenient and accurate choice is a step-function projecting on $k \leq 2 k_{F_{q}}$. This leads to the effective pairing density

$\kappa_{q}^{(\mathrm{eff})}\left(k_{F_{q}}\right)=\int_{0}^{\infty} \frac{d k}{4 \pi^{2}} \frac{k^{2} F(k)}{E_{q}\left(k, \Delta_{q}\right)}$,

and the (approximate) pairing energy density

$\mathcal{E}_{P}(\rho) \approx \sum_{q} \kappa_{q}^{(\mathrm{eff}) *}\left(k_{F_{q}}\right) G_{p a i r}\left(k_{F_{q}}\right) \kappa_{q}^{(\mathrm{eff})}\left(k_{F_{q}}\right)$.

In Fig. 7, the pairing field $\Delta_{q}\left(k_{F_{q}}\right)$ in symmetric nuclear matter is shown, obtained with Eqs. (35) and (37), where $G_{\text {pair }}$ was derived from the singlet-even Bonn NN potential. The field strength reaches a maximum at $\rho \sim 0.04 \mathrm{fm}^{-3}$, that is, one-fourth of the saturation density. Pairing is obviously a low density phenomenon. This is in perfect agreement with many investigations in finite nuclei (see, e.g., [191,192]), where the pairing is found to be effective only in the surface region-which is dominated essentially by the valence particles. The pairing effects contribute only a minor part to the total binding energy but they are clearly seen in two-particle separation energies, i.e., in higher order variations of nuclear binding energies. In weakly bound nuclei, however, the pairing correlations among valence nucleons may play a decisive role for binding [114]. 
The approach sketched above is easily taken over to pairing calculation in finite nuclei. In local density approximation, $k_{F_{q}}(\rho)$ and consequently $G_{\text {pair }}\left(k_{F_{q}}\right)$ convert into the radial-dependent functions. Since $G_{\text {pair }}$ does not depend on momentum, it corresponds to a contact interaction in coordinate-space, rendering the nuclear paring field into a rather simple product form

$\Delta_{q}(r)=G_{\text {pair }}\left(k_{F_{q}}(r)\right) \kappa_{q}(r)$,

which, according to Eq. (39), leads to the pairing energy

$E_{P}^{(\mathrm{GEDF})}=\sum_{q} \int d^{3} r \kappa_{q}^{*}(r) G_{p a i r}\left(k_{F_{q}}(r)\right) \kappa_{q}(r)$,

being a functional of the normal and the anomalous HFBdensities.

\section{Reduction to a heuristic Schrödinger equation}

\subsection{Non-relativistic mean-field approach}

Large scale studies on the structure and production of SHN rely on the availability of flexible theoretical methods, allowing one to explore the parameter space in detail in finite time and with acceptable numerical effort. The approaches based on purely phenomenological input come to their limits, once the region of the known elements is left. Fully self-consistent microscopic approaches require too many resources for their systematic use. However, they provide guidance for extrapolations into unknown territories of the nuclear landscape, although keeping in mind the remaining uncertainties observed e.g. in the variations of mass predictions obtained by competing EDF models. Ideally, a broad collection of results of different EDFs should be used to determine in a statistically significant manner the uncertainty band of the theoretical input and how that affects the derived predictions for SHN. In practice, we are not yet at that stage. Keeping this in mind, here we present two schemes suitable for connecting microscopic and heuristic methods in SHN research. In this section, we start from non-relativistic EDF theory, and show how to reduce the self-consistent meanfield self-energies and the corresponding wave equation to an effective potential and a heuristic single-particle Schrödinger equation of standard form.

In the HFB calculations with finite-range interactions, underlying the GEDF approach, in principle non-local self-energies are encountered because antisymmetrization requires one to use the full one-body density matrices rather than the local number densities. As discussed before and in more detail in Ref. [112], in the GEDF approach the DME of Negele and Vautherin [111] is used as an elegant remedy for that problem. Different from the original work of Negele and
Vautherin, we retain in the GEDF approach the full finiterange structure for the Hartree potential as it is practiced in RMF models. The DME is used to reduce the Fock part of the mean field to local form. This procedure leads to effective proton and neutron masses $M_{q}^{*}(\rho)$, accounting for the leading order momentum dependence. Renormalizations of the Hartree potential are necessary as well, in order to account for the bulk contributions of the Fock terms and, as discussed before, to incorporate unresolved many-body interactions.

In infinite nuclear matter, the DME procedure amounts to approximate the momentum-dependent HF/HFB selfenergies for states inside the Fermi sphere by a $k^{2}$ behavior, which even in the covariant DBHF case [164] works perfectly well. The self-energies of proton $(q=Z)$ and neutron $(q=N)$ states are expanded around their respective Fermi momentum $k_{F_{q}}$,

$\Sigma_{q}(k, \rho) \simeq \Sigma_{q}\left(k_{F_{q}}, \rho\right)+\left.\left(k^{2}-k_{F_{q}}^{2}\right) \frac{\partial \Sigma_{q}(k, \rho)}{\partial k^{2}}\right|_{k_{F_{q}}^{2}}$.

The static potential $\Sigma_{q}\left(k_{F_{q}}, \rho\right)$ includes the isoscalar and isovector short-range central and spin-orbit potentials. In a finite nucleus, the proton potential includes also the Coulomb potential, calculated with the microscopic charge density $\rho_{\mathrm{ch}}(\mathbf{r})$. This adds to the EDF the Coulomb-energy density:

$\frac{1}{2} \mathcal{E}_{\mathrm{C}}=\frac{e^{2}}{2} \int d \mathbf{r} \rho_{\mathrm{ch}}(\mathbf{r})\left(\int d \mathbf{r}^{\prime} \frac{\rho_{\mathrm{ch}}\left(\mathbf{r}^{\prime}\right)}{\left|\mathbf{r}-\mathbf{r}^{\prime}\right|}-\frac{3}{2}\left(\frac{3}{\pi}\right)^{1 / 3} \rho_{\mathrm{ch}}^{1 / 3}(\mathbf{r})\right)$,

where the first term is the Hartree-energy density, followed by the Fock-exchange correction term, evaluated in the Slater approximation [125]. The electric charge is denoted by $e^{2} \simeq$ $1.44 / \hbar$.

Since $\Sigma_{q}\left(k_{F_{q}}, \rho\right)$ is calculated with the HFB densities, paring effects - if present-are accounted for on the level of occupation probabilities. In principle, higher order pairing effects, affecting the shape of the radial wave functions of upper and lower Bogoliubov-components, can be included in $\Sigma_{q}\left(k_{F_{q}}, \rho\right)$ by the pairing self-energies, Eq. (17). However, from this point on, we neglect these minor contributions and solve the Gorkov equations, Eq. (12), in BCS approximation, Eq. (16), but with state-dependent gaps, $\Delta_{q \alpha}=\left\langle\phi_{q \alpha}|\Delta| \phi_{q \alpha}\right\rangle$.

Combining the $k^{2}$-term with the kinetic energy, we obtain the effective HFB mass

$M_{q}^{*}=M_{q}\left(1+\left.\frac{2 M_{q}}{\hbar^{2}} \frac{\partial \Sigma_{q}(k, \rho)}{\partial k^{2}}\right|_{k_{F_{q}}^{2}}\right)^{-1}$ 
and the renormalized and shifted potential

$\tilde{V}_{q}(\rho)=\frac{\hbar^{2} k_{\mathrm{F}_{q}}^{2}}{2 M_{q}}\left(1-\frac{M_{q}}{M_{q}^{*}}\right)+\Sigma_{q}\left(k_{\mathrm{F}_{q}}, \rho\right)$,

where $M_{q}$ is the free nucleon mass. Since the proton and neutron self-energies contain isoscalar and isovector components (see Eq. (6)), the respective effective masses differ in charge-asymmetric matter. Microscopic descriptions like the GEDF and the covariant DDRH approaches lead in a natural manner to that kind of asymmetry $[119,164]$. Purely phenomenological approaches like the Skyrme and most of the RMF models, however, do not account for this effect, mainly because the available data do not allow a safe determination of the corresponding parameters.

In a finite nucleus, densities and Fermi momenta are to be replaced by $\rho_{q}(\mathbf{r})$ and $k_{F_{q}}(\mathbf{r}) \sim \rho_{q}(\mathbf{r})^{1 / 3}$, respectively, leading to radial-dependent potentials and effective masses. The single-particle states $\phi_{q \alpha}$ and energies $e_{q \alpha}$ are the eigenstates and eigenvalues, respectively, of the wave equation

$$
\left(-\nabla \cdot \frac{\hbar^{2}}{2 M_{q}^{*}(\mathbf{r})} \nabla+\tilde{V}_{q}(\mathbf{r})-e_{q \alpha}\right) \phi_{q \alpha}(\mathbf{r})=0
$$

applying to the diagonal parts of the coupled system Eq. (12). Although this equation is very similar to the one used in the phenomenological approaches, the single-particle potential $\tilde{V}_{q}$ cannot be compared directly with the phenomenological single-particle potentials because typically they are determined for the constant free nucleon mass $M_{q}^{*}=M_{q}$. The density dependence of the effective mass $M_{q}^{*}(\mathbf{r})$, however, acts as a renormalization of $\tilde{V}_{q}$ over the nuclear volume. Hence, the reduction of microscopic self-energies to a Schrödinger-equivalent static potential as used in purely phenomenological approaches requires a couple of intermediate steps. As a first step, the kinetic-energy operator in Eq. (46) is rewritten as

$$
\begin{aligned}
& -\nabla \cdot \frac{\hbar^{2}}{2 M_{q}^{*}(\mathbf{r})} \nabla \\
& =\frac{M_{q}}{M_{q}^{*}(\mathbf{r})}\left(-\frac{\hbar^{2}}{2 M_{q}^{*}(r)} \nabla^{2}+\frac{\hbar^{2}}{2 M_{q}^{*}(r)} \boldsymbol{\mu}_{q}(\mathbf{r}) \cdot \nabla\right),
\end{aligned}
$$

where

$$
\boldsymbol{\mu}_{q}(\mathbf{r})=\nabla \ln \left(M_{q}^{*}(\mathbf{r}) / M_{q}\right)
$$

In the case of spherical symmetry, Eq. (46) is transformed into the standard Schrödinger equation

$$
\left(-\frac{\hbar^{2}}{2 M_{q}} \nabla^{2}+\tilde{U}_{q}\left(r, e_{q \alpha}\right)-e_{q \alpha}\right) \phi_{q \alpha}(r)=0
$$

with the state-dependent effective mean-field potential

$$
\begin{aligned}
\tilde{U}_{q}\left(r, e_{q \alpha}\right)= & \frac{M_{q}^{*}(r)}{M_{q}}\left[\tilde{V}_{q}(r)+\frac{\hbar^{2}}{2 M_{q}^{*}(r)} \mu_{q}(r) \frac{\partial}{\partial r}\right. \\
& \left.+\left(\frac{M_{q}}{M_{q}^{*}(r)}-1\right) e_{q \alpha}\right]
\end{aligned}
$$

which in fact is still an operator because the potential $\tilde{U}_{q}\left(r, e_{q \alpha}\right)$ contains a derivative operator and depends on the single-particle energy $e_{q \alpha}$. However, with $\tilde{U}_{q}\left(r, e_{q \alpha}\right)$ one can define the wave equation which is formally of the standard Schrödinger-type equation.

In spherical systems, the gradient term can be treated by standard methods, but it becomes numerically rather involved in deformed nuclei. Hence, a global approach, avoiding such complications but retaining the essential features, is introduced here. We assume that $\phi_{q \alpha}$ is locally an eigenfunction of the momentum operator,

$\boldsymbol{\mu}_{q}(\mathbf{r}) \cdot \nabla \phi_{q \alpha} \approx \mu_{q}(r) k_{\mathrm{eff}}\left(r, e_{q \alpha}\right) \phi_{q \alpha}$,

where $k_{\text {eff }}$ has to be determined. Then Eq. (50) is rewritten as

$$
\begin{aligned}
\tilde{U}_{q}\left(r, e_{q \alpha}\right)= & \frac{M_{q}^{*}(r)}{M_{q}}\left[\tilde{V}_{q}(r)+\frac{\hbar^{2}}{2 M_{q}^{*}(r)} \mu_{q}(r) k_{\mathrm{eff}}\left(r, e_{q \alpha}\right)\right. \\
& \left.+\left(\frac{M_{q}}{M_{q}^{*}(r)}-1\right) e_{q \alpha}\right]
\end{aligned}
$$

From Eqs. (46) and (49) we obtain the local kinetic energy

$\frac{\hbar^{2} k^{2}}{2 M_{q}^{*}(r)}=e_{q \alpha}-\tilde{V}_{q}(r)$

and the state-dependent effective local momentum

$k_{\mathrm{eff}}^{2}\left(r, e_{q \alpha}\right)=\frac{2 M_{q}}{\hbar^{2}}\left(e_{q \alpha}-\tilde{U}_{q}(r)\right)$,

respectively. Using Eqs. (53) and (54), we obtain the quadratic equation

$k_{\mathrm{eff}}^{2}(r, k)=-\mu_{q}(r) k_{\mathrm{eff}}(r, k)+k^{2}$,

to find

$k_{\mathrm{eff}}(r, k)=-\frac{1}{2} \mu_{q}(r)+\sqrt{k^{2}+\frac{1}{4} \mu_{q}^{2}(r)}$.

As seen, the state dependence of $k_{\text {eff }}$ is now represented by the momentum $k$, which lies inside the proton or neutron Fermi sphere. To project out this dependence, we average $k_{\text {eff }}$ over the respective Fermi sphere with local Fermi momentum $k_{\mathrm{F}_{q}}(r)=\left(3 \pi^{2} \rho_{q}(r)\right)^{\frac{1}{3}}$,

$\bar{k}_{\mathrm{eff}}(r)=\frac{3}{k_{\mathrm{F}_{q}}^{3}} \int_{0}^{k_{\mathrm{F}_{q}}} \mathrm{~d} k k^{2} k_{\mathrm{eff}}(r, k)$. 
The substitution of Eq. (56) into Eq. (57) leads to

$$
\begin{aligned}
\bar{k}_{\mathrm{eff}}= & -\frac{1}{2} \mu_{q}+\frac{3}{8 k_{\mathrm{F}_{q}}^{3}}\left[k_{\mathrm{F}_{q}} \sqrt{\frac{1}{4} \mu_{q}^{2}+k_{\mathrm{F}_{q}}^{2}}\left(\frac{1}{4} \mu_{q}^{2}+2 k_{\mathrm{F}_{q}}^{2}\right)\right. \\
& \left.-\frac{1}{16} \mu_{q}^{4} \operatorname{arcsinh}\left(\frac{2 k_{\mathrm{F}_{q}}}{\mu_{q}}\right)\right] .
\end{aligned}
$$

Except for the nuclear surface region one finds $\mu_{q} \ll k_{\mathrm{F}_{q}}$, and the limiting behavior shows that

$\bar{k}_{\mathrm{eff}} \sim \frac{3}{4} k_{\mathrm{F}_{q}}\left[1+\mathcal{O}\left(\frac{\mu_{q}}{k_{\mathrm{F}_{q}}}\right)\right]$.

Finally, we define the state-independent single-particle potential by averaging $\tilde{U}_{q}$ over the local Fermi sphere:

$$
\begin{aligned}
U_{q}(r)= & \frac{3}{k_{\mathrm{F}_{q}}^{3}} \int_{0}^{k_{\mathrm{F} q}} \mathrm{~d} k k^{2} \tilde{U}_{q}(r, k) \\
= & \tilde{V}_{q}(r)+\frac{\hbar^{2}}{2 M_{q}} \mu_{q}(r) \bar{k}_{\mathrm{eff}} \\
& +\frac{3}{5}\left(1-\frac{M_{q}^{*}(r)}{M_{q}}\right) \frac{\hbar^{2} k_{\mathrm{F}_{q}}^{2}(r)}{2 M_{q}^{*}(r)} .
\end{aligned}
$$

The density dependence of $M_{q}^{*}$ induces in the self-consistent approaches a repulsive correction term to the bare selfconsistent mean-field potential $\tilde{V}_{q}$. So, the equivalent potential $U_{q}$ is shallower than the self-consistent bare potential and it is this potential which has to be compared with the phenomenological single-particle potentials. In the case of $M_{q}^{*}=M_{q}$, this correction vanishes and the Schrödingerequivalent potential is reduced to the self-consistent meanfield potential, $U_{q}=\tilde{V}_{q}$. The renormalized self-consistent potential can be regarded as the central potential for the Schrödinger-equivalent equation with bare nucleon mass.

The two kinds of potentials are compared for ${ }^{264} \mathrm{Ds}$ in Fig. 9. The shapes of the self-consistent and the Schrödingerequivalent potentials are almost identical, but the depths are rather different because of the renormalizations effects induced mainly by the momentum dependence hidden in the effective mass.

For the full Schrödinger-equivalent potential, the spinorbit potential and, for protons, the Coulomb potential have to be added. In the scheme indicated above, the microscopic spin-orbit potential is retained, which would not comply with the empirical procedure to use the derivative of the central potential as the spin-orbit potential form factor:

$U_{q}^{(l s)}(r)=-\kappa_{q}\left(\frac{1}{r} \frac{\mathrm{d} U_{q}}{\mathrm{~d} r}\right) \mathbf{l} \cdot \mathbf{s}$,

where $U_{q}$ is spin-independent central potential. The scaling parameter is chosen typically as $\kappa_{q} \approx 0.263(1+2(N-$ $Z) / A$ ) [39], varying between 0.3 and $0.41 \mathrm{fm}^{2}$. With an appropriately adjusted scaling, this approximation can be used also for the Schrödinger-equivalent potential, at the expense of an additional free parameter.

Closing this section, we add a brief discussion on selfenergies with an intrinsic energy dependence like. A consequence of such dispersive polarization couplings is that the energy-momentum dispersion relation is changed. A frequently used solution for that problem is a linearization with respect to the reference energy, leading to another type of effective mass, the so-called $E$ mass $\bar{M}_{q}$. For the dispersive self-energy $\Sigma_{q}^{(d)}(\omega)$, one defines

$\frac{\bar{M}_{q}}{M_{q}}=1-\left.\frac{\partial}{\partial \omega} \operatorname{Re}\left(\Sigma_{q}^{(d)}(\omega)\right)\right|_{\omega=\omega_{F}}$

where typically an expansion with respect to the Fermi energy, $\omega_{F_{q}}$, is chosen. Mahaux and Sartor [193] have investigated in detail the effects of general momentum- and energydependent mass operators on single-particle dynamics and spectral distributions. The linearization procedure applied both to the momentum and energy dependence leads to the generalized effective mass

$M_{q}^{*} \mapsto M_{q}^{*} \frac{\bar{M}_{q}}{M_{q}}$,

which at the Fermi surface approaches the value $M_{q}$. This increases the density of single-particle states at the Fermi surface, a much wanted remedy for the well-known discrepancy between the HF/HFB and the observed density of states.

\subsection{Covariant mean-field approach}

The covariant EDF approaches derive the equation of motions for nucleon and meson fields from the Lagrangian density,

$\mathcal{L}_{C E D F}=\mathcal{L}_{N}(\Psi)+\mathcal{L}_{S}(\Phi)+\mathcal{L}_{v}(V)+\mathcal{L}_{i n t}(\Psi, \Phi, V)$,

including, in the order of appearance, Lagrangian densities for the free motion of nucleon fields $\Psi$, of scalar fields $\Phi$, vector fields $V$, and, most essential, the Lagrangian of the interactions of the fundamental fields. After the introduction of the covariant methods into the nuclear theory, a multitude of the CEDF-Lagrangians has been presented, ranging from the rather simple, but very influential, original scalar-vector model of Walecka and Serot, the Lagrangians modeling in-medium interactions by various kinds of nonlinear self-interactions of scalar and vector fields, and the Lagrangians achieving that goal by the density-dependent meson-nucleon vertex functionals, as discussed before. A comprehensive review of the achievements and perspectives of the CEDF theory is found in the recent review article by Shen et al. [124]. For the present purpose, it is sufficient to 
remember that the final goal of the CEDF model is to derive and solve the equations of motions for nucleons in the RMF approximation, i.e., all scalar and vector fields are treated as condensed classical fields, driven by nucleon sources.

The key quantity of RMF nucleon dynamics is the Dirac equation (in units of $c=1$ )

$$
\begin{aligned}
& \left(\begin{array}{cc}
M_{q}+S_{q}(\mathbf{r})+V_{q}(\mathbf{r}) & \boldsymbol{\sigma} \cdot \mathbf{p} \\
\sigma \cdot \mathbf{p} & -M_{q}-S(\mathbf{r})+V(\mathbf{r})
\end{array}\right)\left(\begin{array}{c}
f_{q \alpha}(\mathbf{r}) \\
g_{q \alpha}(\mathbf{r})
\end{array}\right) \\
& =E_{q \alpha}^{D}\left(\begin{array}{c}
f_{q \alpha}(\mathbf{r}) \\
g_{q \alpha}(\mathbf{r})
\end{array}\right),
\end{aligned}
$$

where $S_{q}$ and $V_{q}$ are the scalar and vector RMF potentials, respectively, both including isoscalar, isovector, and-if relevant-electromagnetic components. The Dirac eigenenergy is often expressed as $E_{q \alpha}^{D}=M_{q}+e_{q \alpha}$ by the reduced Schrödinger-like single-particle energy $e_{q \alpha}$. The upper $\left(f_{q \alpha}\right)$ and lower $\left(g_{q \alpha}\right)$ components of the Dirac state vector are the Pauli-type spin-space wave functions.

We define the effective Dirac mass

$$
M_{q}^{D}(\mathbf{r})=M_{q}+S_{q}(\mathbf{r}),
$$

and the Dirac self-energy

$$
\Sigma_{q}^{D}(\mathbf{r})=S_{q}(\mathbf{r})+V_{q}(\mathbf{r}) .
$$

The projection onto the upper component of the Dirac function results in the equation for $f_{q \alpha}(\mathbf{r})[116,136,194-197]$

$$
\begin{aligned}
& \left\{\boldsymbol{\sigma} \cdot \mathbf{p} \frac{1}{2 M_{q}^{D}(\mathbf{r})-\Sigma_{q}^{D}(\mathbf{r})+e_{q \alpha}} \boldsymbol{\sigma} \cdot \mathbf{p}+\Sigma_{q}^{D}(\mathbf{r})\right\} f_{q \alpha}(\mathbf{r}) \\
& \quad=e_{q \alpha} f_{q \alpha}(\mathbf{r}),
\end{aligned}
$$

and

$g_{q \alpha}(\mathbf{r})=\frac{1}{2 M_{q}^{D}(\mathbf{r})-\Sigma_{q}^{D}(\mathbf{r})+e_{q \alpha}} \boldsymbol{\sigma} \cdot \mathbf{p} f_{q \alpha}(\mathbf{r})$.

In order to connect to the results of the previous section, we introduce as the coordinate-dependent effective mass

$M_{q}^{*}(\mathbf{r})=M_{q}^{D}(\mathbf{r})-\frac{1}{2}\left(\Sigma_{q}^{D}(\mathbf{r})-e_{q \alpha}\right)$.

We note that $M_{q}^{D}-\Sigma_{q}^{D} / 2$ amounts to a magnitude of a few hundred $\mathrm{MeV}$ in the nuclear interior and approaches asymptotically the free-space nucleon mass $M_{q} \sim 940 \mathrm{MeV}$, which is always much larger, by at least one order of magnitude, than the reduced relativistic energy $e_{q \alpha}$. Hence, we can expand in the parameter $e_{q \alpha} /\left(2 M_{q}^{*}\right)$ and safely disregard this kind of state dependence of $M_{q}^{*}(\mathbf{r})$, somewhat simplifying the derivation of the Schrödinger-equivalent equation. Thus, in zeroth order in $e_{q \alpha} /\left(2 M_{q}^{*}\right)$, Eq. (68) is transformed into the compelling form [196]

$$
\begin{aligned}
& {\left[-\nabla \frac{\hbar^{2}}{2 M_{q}^{*}} \nabla+\Sigma_{q}^{D}(\mathbf{r})\right.} \\
& \left.-\frac{\hbar}{2 M_{q}^{* 2}}\left(\nabla M_{q}^{*}\right)(\mathbf{p} \times \boldsymbol{\sigma})\right] f_{q \alpha}(\mathbf{r})=e_{q \alpha} f_{q \alpha}(\mathbf{r}) .
\end{aligned}
$$

Equation (71) formally has the same structure as Eq. (46), where $\Sigma_{q}^{D}$ replaces the previously used bare self-consistent mean field $\tilde{V}_{q}$. The last term of Eq. (71)—enclosed in brackets-deserves special attention. In spherical systems, this term leads immediately to the spin-orbit potential

$V_{q}^{(l s)}(r)=-\frac{\hbar^{2}}{M_{q}^{*}(r)}\left(\frac{1}{r} \frac{\mathrm{d} \ln \left(M_{q}^{*}(r) / M_{q}\right)}{\mathrm{d} r}\right) \mathbf{l} \cdot \mathbf{s}$,

by which we recover the famous RMF result that both the central spin-independent and the spin-orbit potential are determined by the same set of scalar and vector fields. Finally, the spin-orbit potential depends on the effective nucleon mass. Thus, in the RMF approach there is no necessity to introduce an additional scaling parameter $\kappa_{q}$. The number of input entities, however, is the same in the two sectors: In non-relativistic theory, central and spin-orbit potentials are defined independently; in the RMF approach we have two independent classical fields.

The further steps towards a Schrödinger-equivalent equation with constant free nucleon mass $M_{q}$ follow exactly the scheme developed in Sect. 4.1, finally converging in the Schrödinger-equivalent spin-independent effective central potential,

$$
\begin{aligned}
U_{q}(r)= & \Sigma_{q}^{D}(r)+\frac{\hbar^{2}}{2 M_{q}} \mu_{q}(r) \bar{k}_{\mathrm{eff}} \\
& +\frac{3}{5}\left(1-\frac{M_{q}^{*}(r)}{M_{q}}\right) \frac{\hbar^{2} k_{\mathrm{F}_{q}}^{2}(r)}{2 M_{q}^{*}(r)},
\end{aligned}
$$

and spin-orbit potential,

$U_{q}^{(l s)}(r)=-\frac{\hbar^{2}}{M_{q}}\left(\frac{1}{r} \frac{\mathrm{d} \ln \left(M_{q}^{*}(r) / M_{q}\right)}{\mathrm{d} r}\right) \mathbf{l} \cdot \mathbf{s}$.

The spin-independent central potential $U_{q}$ is given as before in terms of $\Sigma_{q}^{D}$ and the modifications due to the momentum dependence which in the RMF case originates from the scalar field - which in fact differs from the vector field by polarization of the Dirac vacuum. The remaining state-dependent effects are treated by averaging over the local Fermi spheres as before. Concluding this section, we recall that the spinorbit interaction is the decisive entity controlling the formation of the nuclear shell structure, with special importance for the definition of shell closures. 


\subsection{EDF for finite nuclei by inversion}

The freedom, but not arbitrariness, in construction of nuclear EDF can be used for introducing and testing new approaches. Here, we present the method approaching the EDF problem from the side of nucleon self-energies. As a guidance, we refer to observations in the non-relativistic GEDF (and Gogny) and covariant EDF approaches where an important conclusion is that finite-range effects play an essential role. For example, in finite nuclei, the RMF-descriptions use Hartree self-energies which take fully into account the finite-range character of meson exchange forces in solving the classical meson field equations. The same is true for the GEDF approach. This is an essential difference from the Skyrme-type EDF method trying to describe the Hartree and Fock interaction terms in an unified manner by momentumdependent contact interactions, thus following closely the original conjecture of Kohn et al. However, it is a simple exercise to show that the folding-type Hartree self-energy-the tadpole diagram in Fig. 1 -leads to a potential $U\left(r, R_{\text {Hartree }}\right)$, which follows the nuclear density but with increased effective radius $R_{\text {Hartree }}=R_{\text {density }}+\delta R$ and similarly for the diffusivity. In order to reproduce these effects by a series of powers of the density or gradients of the density, a large number of terms is required. Hence, a considerable part of the gradient terms in the Skyrme functional will in fact be related to the reconstruction of trivial Hartree folding effects of minor physical significance. The Fock terms, on the other hand, are much more amenable to the description by contact interactions because they are determined by high-momentum components.

Hence, rather than starting with-possibly biased-assumptions on the functional and operator structure of the interaction part of the EDF, we introduce a method by which the proper EDF is reconstructed from phenomenological self-energies by an inversion procedure. In passing, we mention that the present approach is part of the ongoing attempts to reconstruct a Kohn-Sham functional by inversion, but using input from empirically determined densities, see, e.g., [198]. As shown already in Fig. 1, the self-energies, entering into single-particle wave equations, include unavoidably rearrangement parts $\Sigma^{(r)}$ which are generated by the variational derivation of the equations of motion, but are not part of the underlying EDF. They are essential for the proper description of single-particle observables, density profiles and radii and the thermodynamical consistency of the theory [118], but must be removed for the reconstruction of the proper EDF. While for the microscopic approaches, discussed in previous sections, this procedure is inherently built in, it is not obvious from the beginning how to separate an otherwise given self-energy into the proper and the rearrangement self-energy components.
For the present purpose, we introduce the isoscalar and isovector densities, $\rho_{+,-}=\rho_{N} \pm \rho_{Z}$, respectively. As an illustrative example, we choose the interaction energy density as

$\mathcal{E}_{\text {int }}\left(\rho_{+}, \rho_{-}\right)=\frac{1}{2}\left[\rho_{+}^{2} V_{+}\left(\rho_{+}\right)+\rho_{-}^{2} V_{-}\left(\rho_{+}\right)\right]$,

including the isoscalar $\left(V_{+}\right)$and isovector $\left(V_{-}\right)$interaction vertex functionals, plus the Coulomb and spin-orbit parts which for simplicity are not shown here. The first variation leads to the isoscalar and isovector potentials

$$
\begin{aligned}
U_{+}\left(\rho_{+}, \rho_{-}\right)= & \frac{\delta \mathcal{E}_{i n t}}{\delta \rho_{+}} \\
= & \rho_{+} V_{+}\left(\rho_{+}\right)+\left.\frac{1}{2} \rho_{+}^{2} \frac{\partial V_{+}(\rho)}{\partial \rho}\right|_{\rho_{+}} \\
& +\left.\frac{1}{2} \rho_{-}^{2} \frac{\partial V_{-}(\rho)}{\partial \rho}\right|_{\rho_{+}}, \\
U_{-}\left(\rho_{+}, \rho_{-}\right)= & \frac{\delta \mathcal{E}_{i n t}}{\delta \rho_{-}}=\rho_{-} V_{-}\left(\rho_{+}\right) .
\end{aligned}
$$

In $U_{+}$, the first term corresponds to the Hartree folding potential, the remaining terms are the rearrangement potentials generated by the intrinsic density dependence of the interactions. Because the conservation of isospin symmetry inhibits an intrinsic dependence of the interactions on $\rho_{-}$, the isovector potential $U_{-}$is given by the tadpole-folding term alone. The proton and neutron potentials are recovered by

$U_{Z, N}\left(\rho_{+}, \rho_{-}\right)=U_{+}\left(\rho_{+}, \rho_{-}\right) \mp U_{-}\left(\rho_{+}, \rho_{-}\right)$.

Suppose that $U_{Z, N}$ and therefore by Eq. (77) also $U_{+,-}$ are known from other sources, e.g., from a fit to nuclear ground-state properties and that the corresponding singleparticle Schrödinger equations have been solved such that the densities are available. Because the DFT is based on the oneto-one correspondence between Kohn-Sham potentials and densities, one can extract Kohn-Sham potentials within the nuclear inverse Kohn-Sham problem from the experimental neutron and proton densities [198]. Then by inversion we can reconstruct the EDF by means of the self-consistency relations

$$
\begin{aligned}
& \frac{1}{2} \rho_{+}^{2} V_{+}\left(\rho_{+}\right)+\frac{1}{2} \rho_{-}^{2} V_{-}\left(\rho_{+}\right)=I_{+}\left(\rho_{+}, \rho_{-}\right), \\
& \frac{1}{2} \rho_{-}^{2} V_{-}\left(\rho_{+}\right)=I_{-}\left(\rho_{+}, \rho_{-}\right),
\end{aligned}
$$

fixing the interaction vertices $V_{+,-}(\rho)$ and their density dependence by evaluation of the functional integrals

$$
\begin{aligned}
& I_{+}\left(\rho_{+}, \rho_{-}\right)=\int_{0}^{\rho_{+}} d \rho^{\prime} U_{+}\left(\rho^{\prime}, \rho_{-}\right), \\
& I_{-}\left(\rho_{+}, \rho_{-}\right)=\int_{0}^{\rho_{-}} d \rho^{\prime} U_{-}\left(\rho_{+}, \rho^{\prime}\right),
\end{aligned}
$$



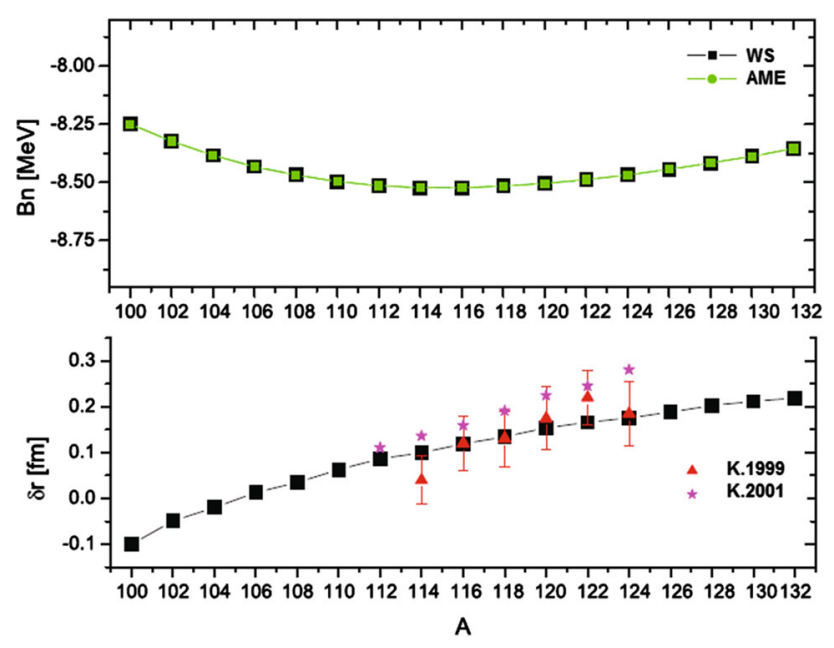

Fig. 8 Binding energy per nucleon $B_{n}=B_{A}$ (upper panel) and neutron skin thickness $\delta r$ (lower panel) for the Sn isotopic chain; see Ref. [199]. Theoretical results of the inversion method, denoted by WS, are compared to AME data [200]. The binding energy data are described by a root-mean deviation of $\Delta B_{n}=49.16 \mathrm{keV}$ per item. The skin thickness data are taken from [201] (K.1999) and [202] (K.2001), respectively

by which we map the-independently determined-potentials to the functional structure of the EDF.

On the level of the EDF, we introduce the Lagrange multipliers $e_{q \alpha}$ and extremalize the functional

$\mathcal{E}^{\prime}\left(\rho_{+}, \rho_{-}\right)=\mathcal{E}\left(\rho_{+}, \rho_{-}\right)-\sum_{q, \alpha} e_{q \alpha}\left|\phi_{q \alpha}\right|^{2} n_{q \alpha}$,

with respect to variation of the Kohn-Sham wave function $\phi_{q \alpha}(\mathbf{r})$ with quantum numbers $(\alpha$ and $q$ ). The occupation number of the corresponding state ( $\alpha$ and $q$ ) is denoted by $n_{q \alpha}=\left\langle A\left|a_{q \alpha}^{\dagger} a_{q \alpha}\right| A\right\rangle \sim \theta\left(\lambda_{q}-e_{q \alpha}\right)$. This leads to the wave equations

$$
\left(-\frac{\hbar^{2}}{2 M_{q}} \nabla^{2}+U_{q}\left(\rho_{+}, \rho_{-}\right)-e_{q \alpha}\right) \phi_{q \alpha}=0
$$

thus closing the self-consistency cycle. The proper nuclear binding energy per nucleon is

$$
\begin{aligned}
B_{A}(N, Z) & =\frac{1}{A} \int d^{3} r \mathcal{E}\left(\rho_{+}(\mathbf{r}), \rho_{-}(\mathbf{r})\right) \\
& =\frac{1}{A} \int d^{3} r\left[\mathcal{E}_{k i n}(\mathbf{r})+\frac{1}{2} \mathcal{E}_{\text {int }}(\mathbf{r})\right],
\end{aligned}
$$

which is considered as a functional of parameters used to define the potential depths and form factors. A practical approach is to start with the WS form factors.

It is worthwhile to point at a few advantages of this inversion method: First, we do not constrain the interactions by a pre-chosen functional ansatz. Second, for phenomenological potentials, determined, e.g., by fitting the separation energies or by extracting from the experimental neutron and proton densities, the inversion approach provides the theoretically well defined scheme to obtain the corresponding nuclear EDF. This is achieved by removing the rearrangement self-energies which are inescapably contained in the phenomenological potentials. Third, the approach is easily extended to deformed nuclei, once the basic EDF parameters are determined for spherical nuclei.

In Fig. 8, the results for the binding energies and the neutron skin thickness of Sn-isotopes are shown. In Ref. [199], the results for other ground-state observables are presented. Nuclear ground-state properties of other isotopic and isotonic chains, respectively, are described with equal quality.

\section{Including microscopic mean-field dynamics into the Mic-Mac approach}

Here, we demonstrate how the effective single-particle potentials obtained from the self-consistent microscopic method modifies the Mic-Mac approach [203] for the SHN. Using advantages of the Mic-Mac approach, we reveal the trends in the shell effects, nuclear binding energies, $Q_{\alpha}$-values, quasiparticle states, and evaporation residue cross-sections. Of great interest for experimental work is the prediction of isomeric states and $\alpha$-decay branchings into higher energy levels. The knowledge of possible spread of the alpha-particle energies, the $\alpha$-decay half-lives, and the terminations of the $\alpha$-decay chains of ${ }^{295} 119$ and ${ }^{295,297} 120$ is important for the experimental identification of these nuclei. Hitherto, all hotfusion reactions for the synthesis of SHN have been performed with beams of ${ }^{48} \mathrm{Ca}$. The new nuclei with $Z \geq 119$ would be hopefully produced using available actinide targets and beams of nuclei heavier than ${ }^{48} \mathrm{Ca}$. The structure of SHN crucially influences the evaporation residue cross-sections in the actinide-based hot-fusion reactions [204]. With the shell closure at $Z \geq 120$, the survival probability of the compound nuclei with $Z=119$ and 120 may be quite high. Employing the dinuclear system (DNS) fusion approach [204-229] and the predictions of the nuclear properties with the MicMac method containing the effective single-particle potentials from the self-consistent HFB approach, we will calculate the production cross-sections of nuclei with $Z=119$ and 120 .

The single-particle potentials from the self-consistent calculations are fitted by WS form factors, Eq. (86). The parameters of WS potential are relevant to consider the nuclear deformation in the Mic-Mac model, where the potential energy is calculated as the sum of two terms $[45,69]$

$E=E_{\mathrm{LDM}}+\delta E_{\mathrm{mic}}$

The first term is a smoothly varying macroscopic energy (the Coulomb and surface energies) calculated with the liquiddrop model. The second term $\delta E_{\text {mic }}$ contains the shell $E_{\mathrm{sh}}$ 

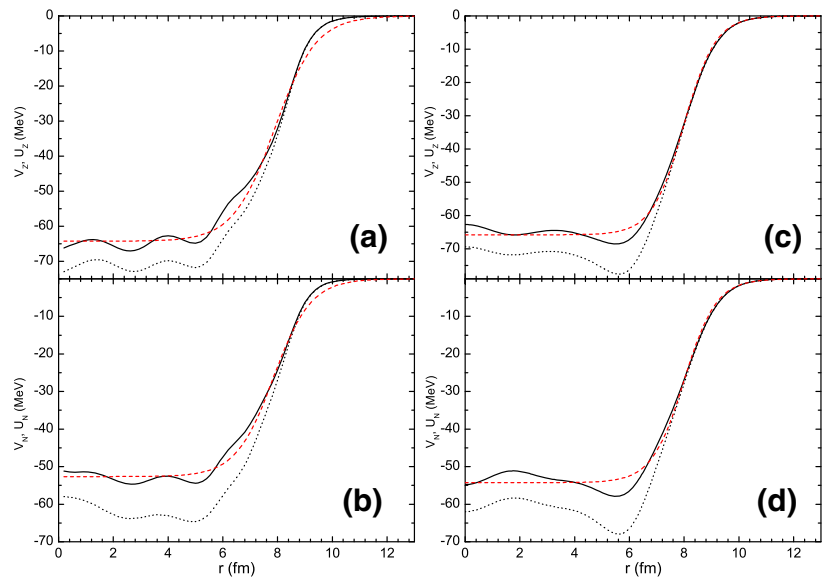

Fig. 9 Single-particle potentials in ${ }^{264}$ Ds: The GEDF/HFB mean-field $V_{q}$ and the resulting Schrödinger-equivalent potentials $U_{q}$ are shown for protons and neutrons in panels (a) and (b), respectively. For comparison, the corresponding RMF potentials $\Sigma_{q}^{D}$ and the derived Schrodingerequivalent potentials $U_{q}^{D}$ are displayed in panels (c) and (d), respectively. The self-consistent mean fields are displayed by dotted lines, the derived Schrödinger-equivalent potentials by a solid lines

and pairing corrections arising due to the shell structure of nucleus. For nuclei with $Z<110$, the absolute values of microscopic corrections obtained in our calculations are close to those obtained in Refs. [45,54-59,230]. The equilibrium deformation of the nucleus corresponds to the position of the minimum on the potential energy surface $E$. As found, the considered isotopes of nuclei with $Z=114$ 120 are almost spherical, i.e., they have the parameters of quadrupole deformation smaller than $|0.15|$.

\subsection{Woods-Saxon parametrization of single-particle potentials}

The Schrödinger-equivalent potentials derived in the Sects. 4.1 and 4.2 are ready for use in any other type of structure calculations. However, they are available only in tabulated, numerical form. In order to match the input needed for the Mic-Mac method as close as possible, it is of advantage to recast these potentials further into a parameterized form in terms of analytical functions of a flexible and easy to handle structure. The method of choice is to parameterize the derived Schrödinger-equivalent potentials by potentials with WS form factors. Once these auxiliary potentials are at hand, they are easily applied in exploratory studies of additional degrees of freedom, e.g. charge asymmetry effects and extrapolations into the region of deformed nuclei.

The indicated procedure is performed for two cases, representative for non-relativistic and covariant EDF approaches. The non-relativistic calculations as input use the HFB mean fields obtained with the up-to-date GEDF version $[113,114]$. For comparison, the RMF self-energies of [152] are used.
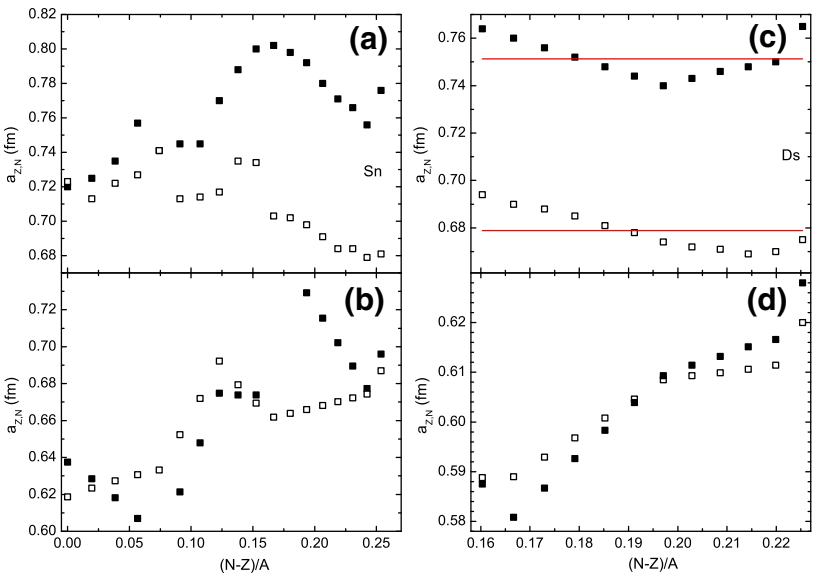

Fig. 10 The diffuseness parameter of the WS potential fitted to the Schrödinger-equivalent potential obtained with the HFB (a, c) and RMF $(\mathbf{b}, \mathbf{d})$ for $\mathrm{Sn}(\mathbf{a}, \mathbf{b})$ and Ds $(\mathbf{c}, \mathbf{d})$ isotopes versus $(N-Z) / A$. The results for protons and neutrons are shown by close and open symbols, respectively. The solid lines (c) show the approximations of $a_{Z, N}$ by constant values

They are obtained with the Lagrangian density NL3 [231], modeling the density dependence of interactions by nonlinear self-interactions of the scalar field. In turn, the (auxiliary) WS potentials are used in the Mic-Mac approach to investigating the shell correction in the ground states of nuclei with $Z=114-120$.

Typical results are displayed in Fig. 9 for the nucleus ${ }^{264}$ Ds. In the nuclear interior, the Schrödinger-equivalent potentials $U_{q}(r)$, Eq. (60), are by about $10 \mathrm{MeV}$ shallower than the bare potentials $V_{q}(r)$, and qualitatively the same observation is made for the RMF-based potentials.

The spherical single-particle potential $U_{q}(r)$ is well reproduced by a standard WS potential

$U_{q}(r)=\frac{V_{q}}{1+\exp \left[\left(r-R_{q}\right) / a_{q}\right]}$,

where $V_{q}=$ const now defines the depth of the potential, $R_{q}=r_{0 q} A^{1 / 3}$ and $a_{q}$ are the radius and diffuseness, respectively, of the potential. The value of $a_{q}$ varies around an average value within $10 \%$ (Fig. 10) over the large interval of $(N-Z) / A$. In the region of the heaviest nuclei of interest, $a_{q}$ approaches in good approximation a constant value with a remaining weak residual dependence on $(N-Z) / A$ (Fig. 10). While the HFB approach provides us with $a_{Z} \approx 0.75 \mathrm{fm}$ and $a_{N} \approx 0.68 \mathrm{fm}$, the RMF approach results in $a_{Z} \approx a_{N} \approx 0.61$ fm for heavy nuclei (Fig. 10). In the phenomenological meanfield potential, the diffuseness parameters are $a_{Z} \approx 0.75 \mathrm{fm}$ and $a_{N} \approx 0.68 \mathrm{fm}$ [39]. The self-consistent calculations result in $r_{0 Z} \approx r_{0 N}=1.22-1.25 \mathrm{fm}$ that is consistent with the phenomenological values $1.24-1.26 \mathrm{fm}$. Although in the phenomenological approaches the parameters of radius $r_{0 q}^{\text {so }}$ and diffuseness $a_{q}^{\text {so }}$ in the spin-orbit interaction are taken equal to those in the central potential, they could be different 


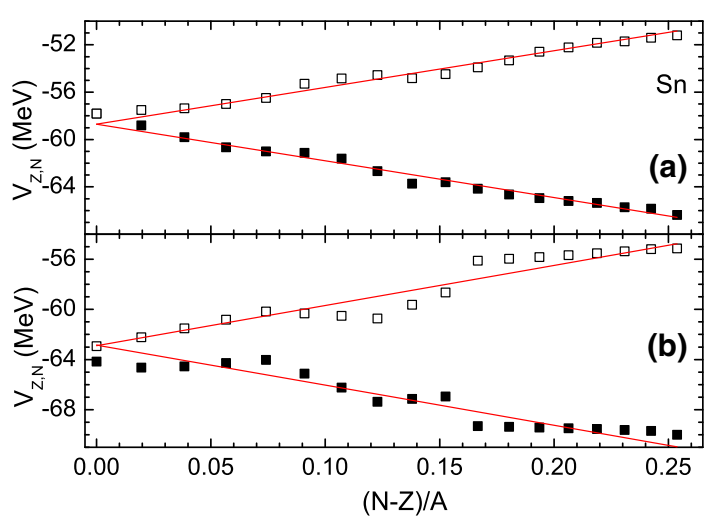

Fig. 11 The extracted depth $V_{Z, N}$ of the mean-field potential versus $(N-Z) / A$ for the isotopes of $\mathrm{Sn}$. The values of $V_{Z, N}$ are obtained with the HFB (a) and RMF (b)

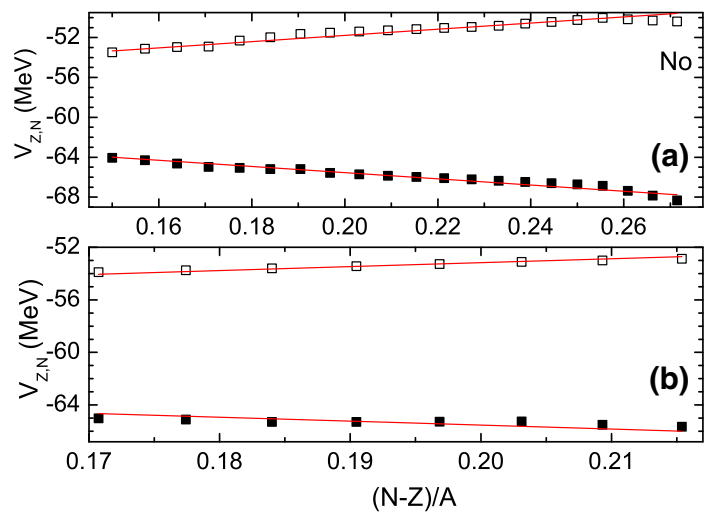

Fig. 12 The same as in Fig. 11, but for the isotopes of No

in the RMF approach. Because $V(r)$ is positive and $S(r)$ is negative, the cancelation between them makes $V(r)+S(r)$ smoother, which could bring a larger diffuseness in the central potential than $V(r)-S(r)$ brings to the spin-orbit potential. It was found that the variations of $r_{0 q}, a_{q}, r_{0 q}^{\text {so }}$, and $a_{q}^{\text {so }}$ within the intervals indicated could change the shell effects as well as shift the position of the shell closure. Below, we will identify parameter sets for which the shell effects are more pronounced at $Z=120$ rather than at $Z=114$.

The Schrödinger-equivalent potentials calculated with the GEDF and RMF approaches were fitted by WS potentials, Eq. (86), in order to study the dependence of the depth $V_{q}$, radius and diffuseness on isospin. In Figs. 11, 12, and 13, the dependencies of $V_{q}$ on $(N-Z) / A$ are presented for the isotopes of Sn, No, and Ds. One can see the almost linear dependence of $V_{q}$ on $(N-Z) / A$. As a result, we suggest the Schrödinger-equivalent potential in the Woods-Saxon form with the parameters listed in Tables 2 and 3. Although the values of $r_{0 q}$ and $a_{q}$ vary with $N$ in the isotopic chain, one can introduce their average values to be used for a certain region of nuclei. The RMF approach provides us with deeper mean-field potentials than the HFB approach. However, in the region of the heaviest nuclei this difference diminishes. In comparison with the self-consistent approaches, in the phenomenological "standard" approach [39] the smaller depth,

$V_{q}=-\left(54 \pm 39.6 \frac{N-Z}{A}\right) \mathrm{MeV}$,

of the WS potential is used for the heaviest nuclei. The plus sign corresponds to $q=Z$ and the minus sign corresponds to $q=N$. For example, the HFB results in $V_{Z}=-64.42 \mathrm{MeV}$ and $V_{N}=-52.18 \mathrm{MeV}$ for ${ }^{272} \mathrm{Ds}$, but from Eq. (87) we obtain -61.57 and $-46.43 \mathrm{MeV}$, respectively. So, the difference of $V_{N}$ is almost twice as large as the difference of $V_{Z}$. The isospin dependence of the mean-field potential is stronger in the phenomenological approach. The proton and neutron single-particle potentials are expressed in terms of isoscalar $\left(U_{0}\right)$ and isovector $\left(U_{1}\right)$ potentials related to total $\rho_{Z}+\rho_{N}$ and isovector $\rho_{N}-\rho_{Z}$ densities, respectively. Thus, the single-particle potential $U_{q}=-\left(V_{0} f_{0}(r) \pm \frac{N-Z}{A} V_{1} f_{1}(r)\right)$ is expressed in terms of the radial form factors of $U_{0}$ and $U_{1}$, which could be different. Leaving this difference for further study, we assume here $f_{0}(r) \approx f_{1}(r)$ and consider $U_{q}$ in the form (86) with $V_{q}=-\left(V_{0} \pm \frac{N-Z}{A} V_{1}\right)$.

To check the quality of the WS potential extracted from the self-consistent approaches, we compare the rms radius of ${ }^{272} \mathrm{Ds}, 6.104,5.947$, and $5.955 \mathrm{fm}$ obtained with the HFB, RMF, and the WS potentials with the parameters from Tables 2 and 3, respectively. As seen, these values are pretty close.

In the expressions for the macroscopic nuclear energy there is a term $a_{\text {sym }} \frac{(N-Z)^{2}}{A}$. The coefficient $a_{\text {sym }}$ is related to the isospin-asymmetric part $V_{1}$ of the potential depth $V_{q}=V_{0} \pm V_{1} \frac{N-Z}{A}, V_{1}=a_{\text {sym }}$ [50]. In Refs. [38,39,45], the value of $a_{\text {sym }}$ varies from 24 to $31 \mathrm{MeV}$. In the "Universal" WS potential [46] as well as in the WS potential used in the QPM [41-43,232-236], the value of $V_{1}$ is about 40 $\mathrm{MeV}$. If the "Universal" WS potential is used to fit the data set of orbital energies for magic nuclei [49], the value of $V_{1}$ becomes about $33.6 \mathrm{MeV}$. The WS potentials with almost the same values of $V_{1}$ were suggested in Refs. [37,237].

So, for the heaviest nuclei we can use

$V_{q}=-\left(58.3 \pm 32 \frac{N-Z}{A}\right) \mathrm{MeV}$

obtained for Ds isotopes as well as for heavier nuclei. Compared to the phenomenological approach the self-consistent ones result in a 5-7 MeV deeper mean-field potential. The deeper potential well leads at least to smaller level density near the Fermi surface and could change the shell correction to the binding energy.

In Figs. 14 and 15, we present the comparison of the singleparticle levels of ${ }^{208} \mathrm{~Pb}$ calculated with the phenomenological WS parameters and WS potentials extracted from the HFB 
Table 2 Parameters of the WS potentials which approximate the Schrödinger-equivalent potentials calculated with the HFB approach for the isotopes of Sn, No, Ds, and 120. The strengths $\kappa_{q}$ of the spin- orbit potential are listed to be used in Eq. (61). The parameters $r_{0 q}^{\text {so }}$ and $a_{q}^{\text {so }}$ of the spin-orbit potential are the same as $r_{0 q}$ and $a_{q}$

\begin{tabular}{llllllr}
\hline Nucleus & $\begin{array}{l}V_{q} \\
(\mathrm{MeV})\end{array}$ & $\begin{array}{l}r_{0 N} \\
(\mathrm{fm})\end{array}$ & $\begin{array}{l}r_{0 Z} \\
(\mathrm{fm})\end{array}$ & $\begin{array}{l}a_{N} \\
(\mathrm{fm})\end{array}$ & $\begin{array}{l}a_{Z} \\
(\mathrm{fm})\end{array}$ & $\begin{array}{l}\kappa_{N} \\
\left(\mathrm{fm}^{2}\right)\end{array}$ \\
\hline Sn & $-\left(58.7 \pm 31 \frac{N-Z}{A}\right)$ & 1.24 & 1.25 & 0.71 & 0.77 & 0.38 \\
No & $-\left(58.6 \pm 34 \frac{N-Z}{A}\right)$ & 1.24 & 1.25 & 0.67 & 0.77 & 0.38 \\
Ds & $-\left(58.3 \pm 32 \frac{N-Z}{A}\right)$ & 1.24 & 1.25 & 0.68 & 0.75 & 0.38 \\
120 & $-\left(58.3 \pm 32 \frac{N-Z}{A}\right)$ & 1.24 & 1.25 & 0.68 & 0.75 & 0.32 \\
\hline
\end{tabular}

Table 3 Parameters of the Woods-Saxon potentials which approximate the Schrödinger-equivalent potentials calculated with the RMF approach for the isotopes of Sn, No, and Ds. The strengths $\kappa_{q}$ of the spin-orbit potential and the parameters $r_{0 q}^{\text {so }}$ and $a_{q}^{\text {so }}$ are listed to be used in Eq. (61)

\begin{tabular}{|c|c|c|c|c|c|c|c|c|c|c|c|}
\hline Nucleus & $\begin{array}{l}V_{q} \\
(\mathrm{MeV})\end{array}$ & $\begin{array}{l}r_{0 N} \\
(\mathrm{fm})\end{array}$ & $\begin{array}{l}r_{0 Z} \\
(\mathrm{fm})\end{array}$ & $\begin{array}{l}a_{N} \\
(\mathrm{fm})\end{array}$ & $\begin{array}{l}a_{Z} \\
(\mathrm{fm})\end{array}$ & $\kappa_{N}$ & $\kappa_{Z}$ & $\begin{array}{l}r_{0 N}^{\mathrm{so}} \\
(\mathrm{fm})\end{array}$ & $\begin{array}{l}r_{0 Z}^{\mathrm{so}} \\
(\mathrm{fm})\end{array}$ & $\begin{array}{l}a_{N}^{\mathrm{so}} \\
(\mathrm{fm})\end{array}$ & $\begin{array}{l}a_{Z}^{\mathrm{so}} \\
(\mathrm{fm})\end{array}$ \\
\hline Sn & $-\left(63.5 \pm 34 \frac{N-Z}{A}\right)$ & 1.23 & 1.22 & 0.66 & 0.67 & 0.27 & 0.21 & 1.1 & 1.1 & 0.45 & 0.48 \\
\hline No & $-\left(59.8 \pm 32 \frac{N-Z}{A}\right)$ & 1.25 & 1.25 & 0.6 & 0.6 & 0.24 & 0.19 & 1.2 & 1.2 & 0.39 & 0.39 \\
\hline Ds & $-\left(59.8 \pm 33 \frac{N-Z}{A}\right)$ & 1.25 & 1.25 & 0.61 & 0.61 & 0.23 & 0.19 & 1.18 & 1.18 & 0.39 & 0.39 \\
\hline
\end{tabular}

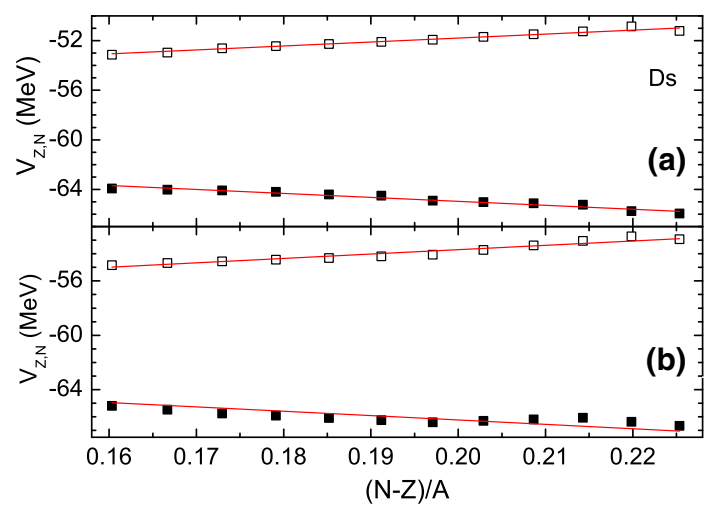

Fig. 13 The same as in Fig. 11, but for the isotopes of Ds

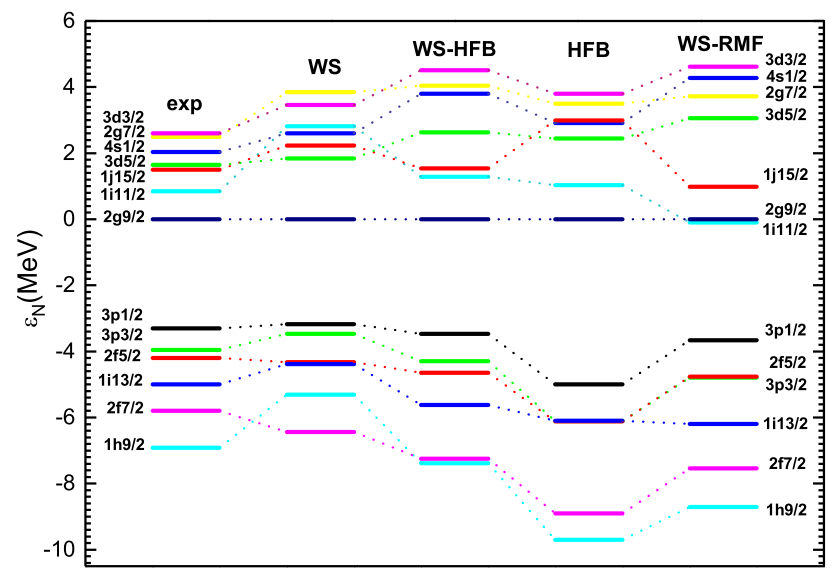

Fig. 14 The experimental (exp) [238,239] neutron single-particle states in ${ }^{208} \mathrm{~Pb}$ are compared with the spectra calculated with phenomenological WS potential (WS), the WS potential extracted from the HFB (WS-HFB), RMF (WS-RMF). The HFB calculations are also shown

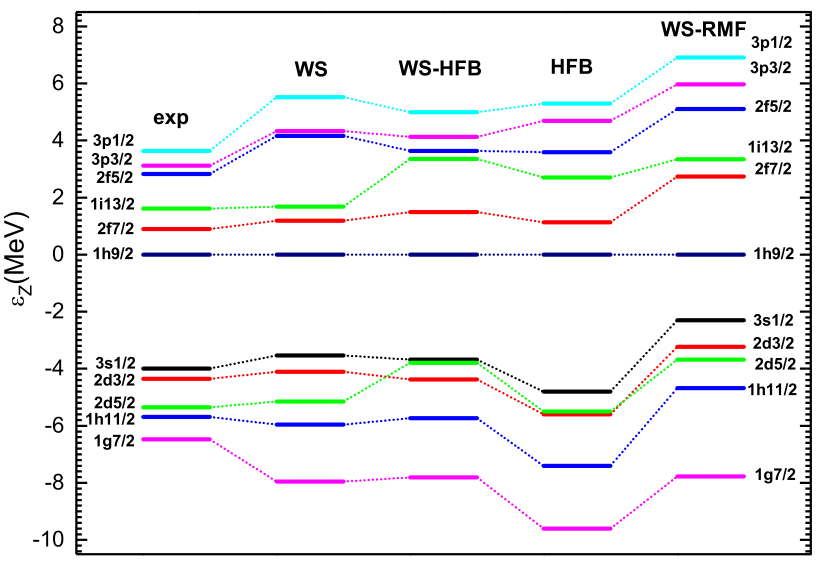

Fig. 15 The same as in Fig. 14, but for proton single-particle states in ${ }^{208} \mathrm{~Pb}$

and RMF approaches. The HFB calculations and experimental data [238,239] are also shown. The WS parameters from the HFB and RMF calculations allow us to describe well the order of almost all levels. As is seen in Figs. 14 and 15, the WS parameters from the HFB and RMF calculations provide similar quality of the description of the single-particle spectra like the phenomenological WS parameters. Because of the smaller effective nucleon mass in the RMF calculations, the corresponding parameters result in the rarefied single-particle spectra. The calculated single-particle spectra marked as WS-RMF in Figs. 14 and 15 can be compared with those obtained in the exact calculations with the NL3 effective interaction [129,240]. For protons, the WS-RMF spectrum in Fig. 15 looks similar although the relative spacing of the levels is not exactly reproduced. For neutrons in 


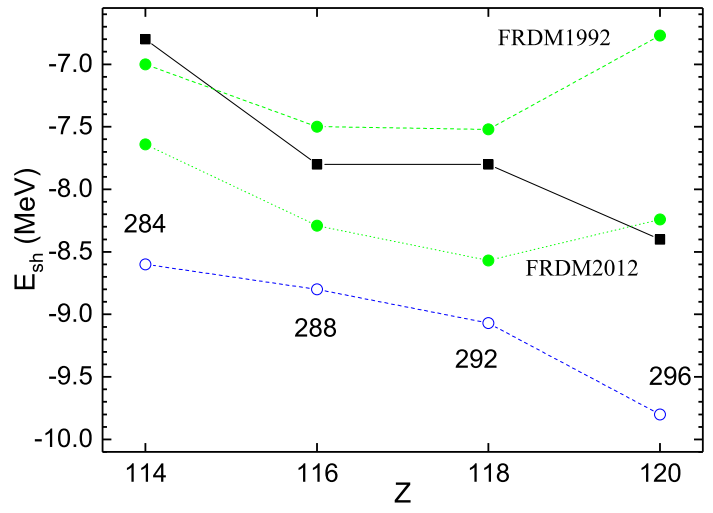

Fig. 16 The calculated ground-state shell corrections in the nuclei of $\alpha$-decay chain of ${ }^{296} 120$. The results are obtained with the Mic-Mac method using the TCSM potential (solid squares), the WS potential extracted from the self-consistent consideration (open circles). The microscopic corrections from the FRLDM1992 and FRLDM2012 [45] are shown by closed circles for comparison. The lines are drown to guide the eye

Fig. 14, the sequence of the levels below the Fermi surface is well reproduced. However, the interval of about $0.1 \mathrm{MeV}$ between the $2 g_{9 / 2}$ and $1 h_{11 / 2}$ levels in the WS-RMF spectrum is smaller than that of about $0.6 \mathrm{MeV}$ in Refs. [129,240]. Though the $1 j_{15 / 2}$ level is about $1.5 \mathrm{MeV}$ below that in Refs. $[129,240]$, it becomes closer to the experiment in Fig. 14. The shell effects are described in a similar way to the WS-RMF and NL3 calculations. In spite of the imperfections of the mapping of the exact NL3 spectra into the WS-RMF spectra, the WS-RMF results remain all shell peculiarities and are suitable for use in the Mic-Mac approaches.

In the non-relativistic and relativistic models, the singleparticle level densities and the pairing gap are correlated with the effective mass [241]. The inclusion of collective vibrations and their coupling to the single-particle or quasiparticle motion increases the level density around the Fermi surface and improves the description of experimental spectra [240249]. The momentum dependence in the Skyrme interaction tends to make the pairing gaps too low [250]. The renormalization due to the spin modes $[248,251]$ and the three-body force [252] corrects the gap by a few hundreds $\mathrm{keV}$. In the whole calculation the essential condition is the correct values of the single-particle energies near the Fermi level [241].

\subsection{Shell effects in $\alpha$-decay chains of $Z=120$ nuclei}

Using parameters given in Eq. (88) and other parameters of the WS potential extracted from the HFB approach, the ground-state shell corrections were calculated for the nuclei of $\alpha$-decay chain $296,298,300,302120$ [169]. In the Mic-Mac calculations, the strength $\kappa_{q}$ of the spin-orbit interaction (Table 2) is set to keep the absolute value in Eq. (61) like in the phenomenological approach. The ground-state defor-

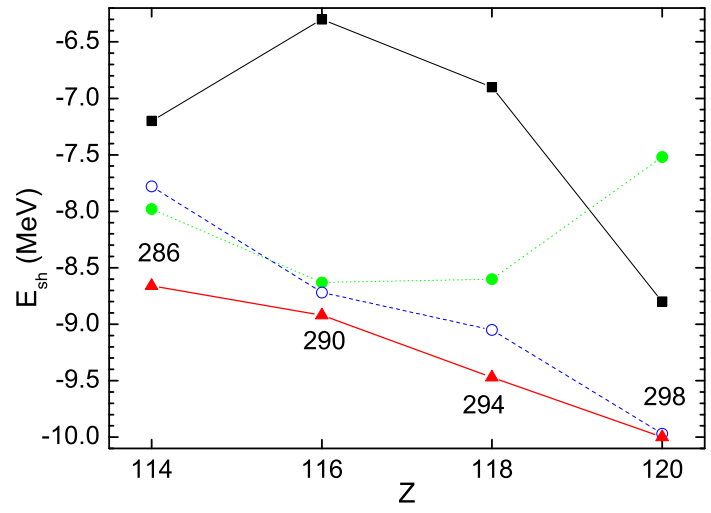

Fig. 17 The calculated ground-state shell corrections in the nuclei of $\alpha$-decay chain of ${ }^{298} 120$. The results are obtained with the Mic-Mac method using the TCSM potential (solid squares), the WS potential extracted from the self-consistent consideration (open circles), and the phenomenological WS potential (closed triangles). The microscopic corrections from the FRLDM2012 [45] are shown by closed circles for comparison. The lines are drown to guide the eye

mations are calculated by minimizing the sum of microscopic and macroscopic parts of the potential energy [253,254]. The basis of the Mic-Mac two-center shell model (TCSM) $[44,68,69]$ is used and the pairing and Strutinsky shell corrections [29] are taken into account. The single-particle states of the lower part of the TCSM spectrum for each deformation $\beta_{2}$ and $\beta_{4}$ are replaced by the corresponding states of the WS potential. As shown in Ref. [203], the energy spectra in these potentials almost coincide. The shape parametrization in the TCSM effectively contains many even multipole moments. Because only the ground-state properties are considered, the disregard of multipole moments with $l \geq 6$ in the WS potential is a suitable approximation for nuclei with relatively small quadrupole deformation and strong shell effects. As shown in Ref. [255] for ${ }^{270} \mathrm{Hs}$, a decisive role, following after $\beta_{2}$, is played by $\beta_{4}$ in these nuclei. To consider the fission path, the higher multipole moments would be required. The monopole pairing is included and treated in the BCS approximation with constant, state-independent pairing gaps. As found, the considered isotopes of nuclei with $Z=114$ 120 are almost spherical, i.e., they have the parameters of quadrupole deformation smaller than 0.15 . The absolute values of the Strutinsky shell corrections become larger towards $Z=120$ (Figs. 16, 17). This is in accordance with the results of self-consistent calculations in which the shell effects are stronger at $Z=120$ rather than at $Z=114$. Indeed, the shell closures are predicted at $Z=114$ and $N=184$ in the phenomenological shell models [45]. However, in the relativistic continuum Hartree-Bogoliubov (RCHB) theory the magicity of $Z=114$ is not at all obvious, in agreement with the most RMF investigations [131]. Only with the effective interaction NL-RA1, the nucleus ${ }^{298} 114$ is expected to be the doubly magic nucleus in the RMF plus BCS calcula- 
tion [256]. Within the SkHF approach, only SkI4 prefers the doubly magic nucleus at $Z=114$ and $N=184$, which is not supported by other Skryme forces such as SkM*, SkP, SkI1, SkI3, SLy6, and SLy7 [257,258]. The shell closure at $Z=120$ is strongly supported in many RCHB calculations. The doubly magic characteristic of ${ }^{292} 120$ has been addressed within the RMF theory [256-259] as well as the effective field theory [260]. Most SkHF investigations also suggest that this nucleus is doubly magic except for SkP $[257,258]$. Due to the strong shell quenching effect in the heaviest nuclei, the doubly magic candidate is interaction dependent, and more experimental data are required to set the parameters. Several Skyrme HF investigations with the effective interactions such as SkP, SkM*, SLy6, and SLy7 predict a proton magic number at $Z=126[257,258,261]$. However, this conclusion is not supported by the RMF calculations.

The calculations $[66,68,69]$ with the TCSM [44] provide us stronger shell effects at $Z=120$ than at $Z=114$ (Figs. 16, 17 ) in the case when the parameters of the TCSM are adjusted for describing the low-lying quasiparticle states. The ground-state shell corrections resulting from the TCSM for $Z=120$ nuclei are close to those obtained with the WS single-particle potential extracted from the HFB selfconsistent approach. For comparison, in Figs. 16 and 17 we present the microscopic corrections obtained with the finiterange liquid-drop model (FRLDM) [45] for the nuclei of $\alpha$ decay chains of ${ }^{296,298} 120$. The parameters of the FRLDM are adjusted for the best description of binding energies of known nuclei. More experimental data for heavy nuclei appeared last years. So, the shell effects beyond $Z=114$ are stronger in the latest version (FRLDM2012) of the model [45] than in the previous one (FRLDM1992); see Fig. 16. However, for $Z=120$ nucleus the values of $\left|E_{\mathrm{sh}}\right|$ remain to be smaller in the FRLDM than those resulting from the other models mentioned in Figs. 16 and 17. The shell corrections obtained with the WS potential extracted here from the HFB approach with the D3Y interaction are pretty close to those obtained in the direct HFB calculations with other EDF [262]. Our single-particle spectra are also consistent with those obtained in the direct RMF calculations [240].

The phenomenological WS single-particle potential with (87) and other parameters adjusted in Refs. [41-43] to describe the low-lying quasiparticle states results in the increase of the shell effects toward $Z=120$ nucleus (Fig. 18). As seen, the shell effects become slightly stronger with decreasing depth of the potential well. Note that the shell structure in the WS model depends also on the ratio between $r_{0 q}$ and $a_{q}$ [203]. In comparison to "standard" values, the values of $a_{N}$ for $U_{q}(r)$ are smaller in the RMF consideration. So, with the extracted $U_{q}(r)$ we obtain different shell effects in the nuclei with $Z=114-120$ than those predicted with the "standard" values of parameters. In the region of well-studied

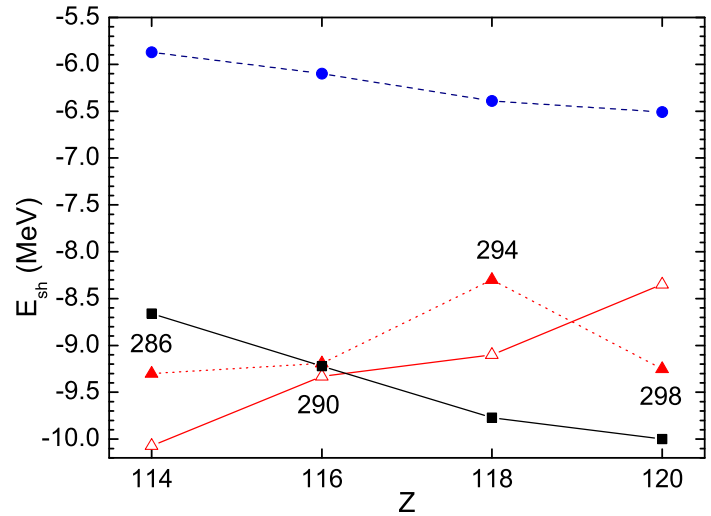

Fig. 18 The calculated ground-state shell corrections in the nuclei of $\alpha$-decay chain of ${ }^{298} 120$. The results are obtained with the Mic-Mac method using the WS potential and spin-orbit strength adopted in Refs. [41-43] (closed squares). The results with 20\% larger and smaller spinorbit strengths are presented by closed and open triangles, respectively. The results obtained with the Mic-Mac method using the WS potential and spin-orbit strength extracted from the self-consistent RMF consideration are shown by closed circles. The lines are drown to guide the eye

nuclei our results are similar to those obtained with the "standard" parameters of the WS model. In Fig. 18, the dependence of $E_{\mathrm{sh}}$ on the strengths $\kappa_{q}$ of spin-orbit interactions is demonstrated at the unchanged other parameters of the phenomenological WS potential. As seen, the $20 \%$ change of $\kappa_{q}$ creates the qualitative difference in the trend of the shell effects. While the decrease of $\kappa_{q}$ results in the weaker change of $E_{\mathrm{sh}}$ from $Z=114$ to $Z=120$, its increase causes the shift of the shell closure from $Z=120$ to $Z=114$. There is certain interval of $\kappa_{q}$ and other parameters in which the shell effects become stronger toward $Z=120$ nucleus. So, the position of the proton shell closure beyond lead crucially depends of $\kappa_{q}$ and $a_{q}$. If $\kappa_{N}$ and $\kappa_{Z}$ are close to $0.23-0.38$ $\mathrm{fm}^{2}$ and $0.23-0.32 \mathrm{fm}^{2}$, respectively, and $a_{Z} \approx 0.75 \mathrm{fm}$ and $a_{N} \approx 0.68 \mathrm{fm}$, the strongest shell effects are expected at $Z=120$ rather than at $Z=114$.

The RMF extraction of the spin-orbit interaction (Table 3) leads to about 1.5 times smaller diffuseness of $\frac{1}{\kappa_{q} M_{q}} \ln \left(M_{q}^{*}(r) / M_{q}\right)$ in Eq. (72) than one of the central potential $U_{q}(r)$. The radius parameters in Eq. (72) are about 5\% smaller than those in $U_{q}(r)$. Because $V$ and $S$ have small diffuseness and different signs, the function $V-S$ has also small diffuseness. For heavy nuclei, the strengths $\kappa_{N} \approx 0.23 \mathrm{fm}^{2}$ and $\kappa_{Z} \approx 0.19 \mathrm{fm}^{2}$ of the spin-orbit interaction are obtained from the RMF approach as the ratio of the derivatives of $U_{q}$ and $\frac{1}{\kappa_{q} M_{q}} \ln \left(M_{q}^{*}(r) / M_{q}\right)$ at $r=r_{0 q}^{\mathrm{so}} A^{1 / 3}$. These values of $\kappa_{q}$ are smaller than those in the HFB and phenomenological calculations. As seen in Fig. 18, the strong reduction of the diffuseness in $U_{l s}^{q}(r)$ and smaller diffusion of $U_{q}(r)$ in comparison with the HFB result in smaller $\left|E_{\mathrm{sh}}\right|$ at the ground states. The shell effects vary smaller from $Z=114$ 
to $Z=120$ where the shell is stronger as in the phenomenological and HFB approaches presented.

We note that the dependencies of $E_{\mathrm{sh}}$ on $Z$ at $Z=114$ 118 are approximately equidistant in almost all Mic-Mac approaches considered. This means that these approaches describe the $Q_{\alpha}$ in a similar way. The largest difference in the trend of $E_{\mathrm{sh}}$ is for nuclei with $Z=120$. Indeed, for these nuclei the existing predictions are varied and the experimental data are required to select the best microscopic approach. We noted that the ground-state deformations are very close (within 10\%) in all approaches used. There are different parametrizations for the HFB and RMF. They can result in different structures of heavy nuclei. So, the level order depends on the input of self-consistent approach. However, the shell closure at $Z=120$ is strongly supported in almost all self-consistent approaches. The neutron shell at $N=184$ is interaction dependent. We consider the nuclei for which the self-consistent approaches indicate stronger shell effects at $Z=120$ than at $Z=114$, opposite to the known Mic-Mac approaches. As the first step, we show the possibility to get similar results with the WS model and relate it to the self-consistent models.

It was found that the self-consistent approaches considered provide deeper single-particle potentials that those used in the phenomenological consideration [37-39,41-43]. The spin-orbit interaction potential extracted from the RMF model appears to be in the form like in the phenomenological studies, with close strength but smaller diffuseness. As seen, the use of these potentials leads to the increase of the shell effects towards $Z=120$ and $N=178-184$. Thus, there is certain interval of parameters of the mean-field potential in the phenomenological Mic-Mac approaches which endorses the stronger shell effects at $Z=120$ rather than at $Z=114$.

The mean-field potentials were extracted for spherical nuclei. The introduction of deformation dependence in these potentials is quite easy. So, the extracted WS potential can be used in the Mic-Mac calculations which seem to be easier in some cases than the self-consistent ones.

\subsection{Mass excesses and $Q_{\alpha}$-values}

The calculated mass excesses $M_{\text {th }}$ and $Q_{\alpha}$-values are presented in Figs. 19 and 20 for nuclei with $112 \leq Z \leq 120$. We treat only the isotopes of SHN which can be reached in complete fusion reactions with available projectiles and targets. As is seen in Fig. 20, the calculated $Q_{\alpha}$ are in a good, within $\sim 300 \mathrm{keV}$, agreement with the available experimental $Q_{\alpha}^{\text {exp }}$ $[1,5,11,17]$. The shell effects at $Z=114$ and $N=172-176$ provide rather weak dependence of $Q_{\alpha}$ on $N$. The strong role of the shell at $N=182$ is reflected in the well pronounced decrease of $Q_{\alpha}$ at $Z=120$. As in the calculations presented, the strong evidence of the shell closure at $Z=120$ and $N=182-184$ was observed within the Mic-Mac approach
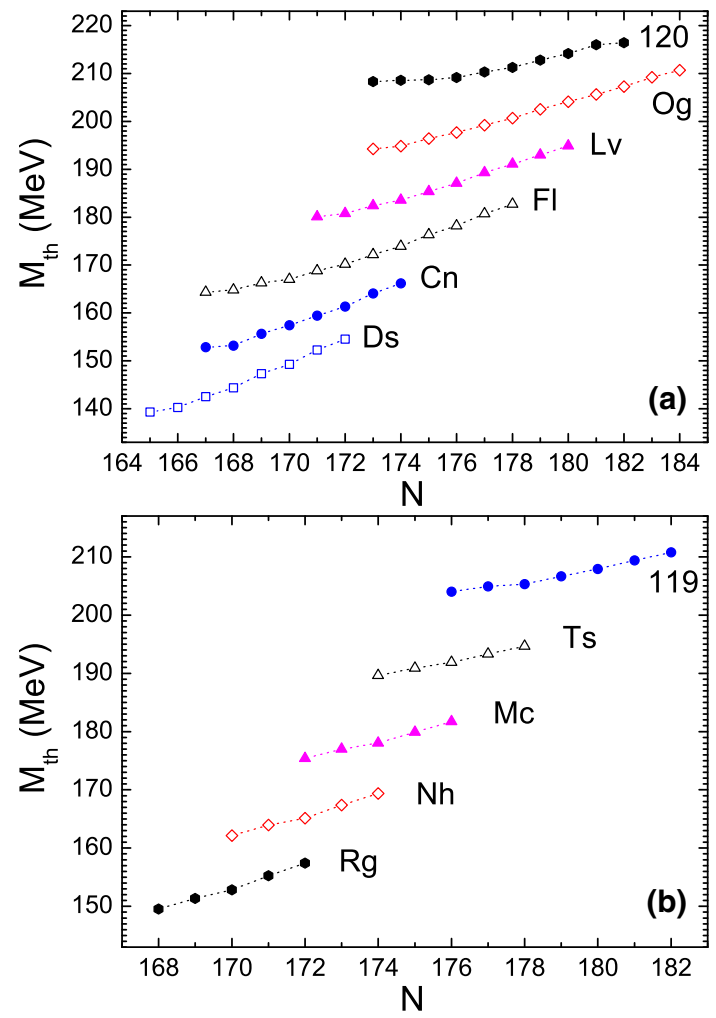

Fig. 19 Calculated mass excesses (symbols connected by lines) for even- $Z$ (a) and odd- $Z$ (b) nuclei with $110 \leq Z \leq 120$. The MicMac method is used with the WS potential extracted from the HFB consideration

of Ref. [69]. For comparison, the $Q_{\alpha}$-values predicted with Mic-Mac model (FRDM2012) [230] are shown in Fig. 21. In contrast to Fig. 21, the $Q_{\alpha}$-value dependence on $N$ is rather flat for the nuclei with $Z=116-120$. The weak shell effects at $Z=120$ and $N=182$ are also seen.

Note that in Fig. 20 the nuclei ${ }^{295} 119$ and ${ }^{295,297} 120$ are expected to have $Q_{\alpha}$-values of about 11.9 and 12.0, 11.5 $\mathrm{MeV}$, respectively. The Mic-Mac model (FRDM2012) [230] results in $Q_{\alpha}=12.9 \mathrm{MeV}$ for ${ }^{295} 119$ and $Q_{\alpha}=13.5,13.7 \mathrm{MeV}$ for ${ }^{295,297} 120$. So, there is a strong difference (about $1-2$ $\mathrm{MeV}$ ) between the $Q_{\alpha}$-values of the models with the phenomenological and self-consistent mean-field potentials.

\subsection{Quasiparticle levels in nuclei of $\alpha$-decay chain of ${ }^{295} 119$ and ${ }^{295,297} 120$}

For the nuclei produced in the $\alpha$-decay chains of ${ }^{295} 119$ and ${ }^{295,297} 120$, the ground-state shell corrections calculated with the mean-field potential extracted here from the HFB approach [112] with the D3Y interaction are presented in Figs. 22 and 23. It was found that the absolute values of the Strutinsky shell corrections become larger towards $Z=120$. This is in accordance with the results of self-consistent cal- 

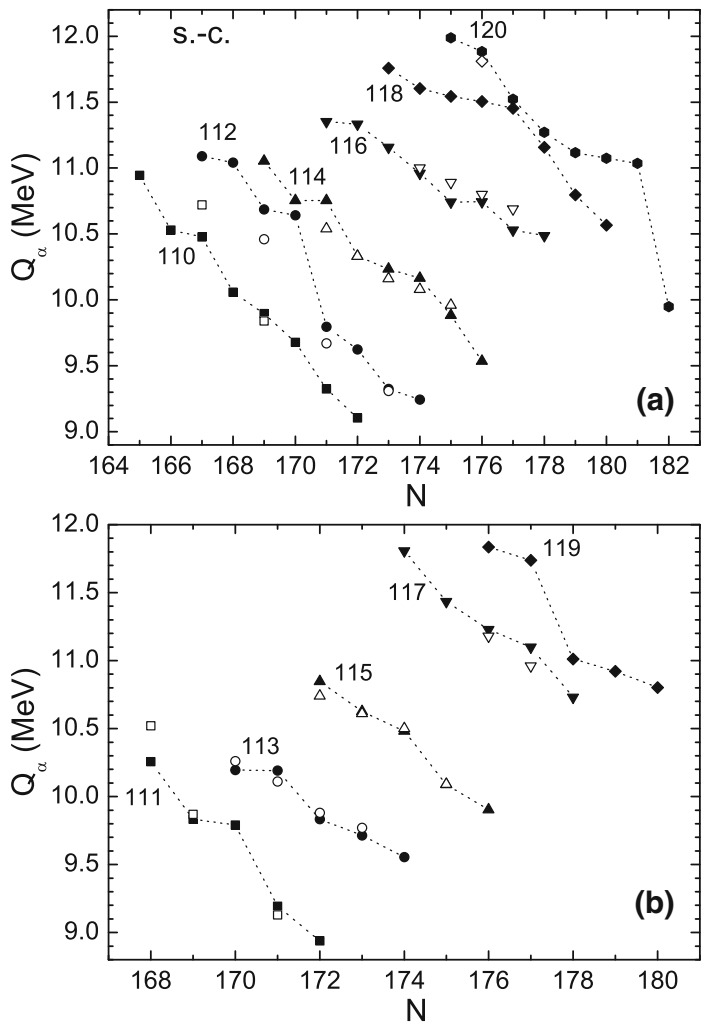

Fig. 20 Calculated $\alpha$-decay energies (closed symbols connected by lines) are compared with available experimental data (open symbols) $[1,5,11,17]$ for even- $Z$ (a) and odd- $Z$ (b) nuclei with $110 \leq Z \leq 120$. The Mic-Mac method is used with the WS potential extracted from the HFB consideration

culations in which the shell effects are stronger at $Z=120$ rather than at $Z=114$.

The shell corrections are pretty close to those obtained in the pure HFB calculations. The ground-state shell corrections resulting from the HFB are also close to those obtained with the Mic-Mac method using the modified TCSM potential in the case when the parameters of the TCSM are adjusted for describing the known low-lying quasiparticle states of the heaviest nuclei $[66,67,69]$. The calculations obtained with the TCSM provide us also stronger shell effects at $Z=120$ than at $Z=114$

For comparison, in Figs. 22 and 23 we present the microscopic corrections obtained with the FRDM2012 [230]. The parameters of the FRDM2012 are adjusted for the best global description of binding energies of known nuclei. For the $Z=120$ nucleus, the value of $\left|E_{\mathrm{sh}}\right|$ remains to be smaller in the FRDM2012 than that resulting from the HFB approach (Fig. 23). The nuclei with $Z=116$ and 118 have the maximum absolute values of the shell corrections in the FRDM2012 model.

For the nuclei with $Z=119$ and 120 , the existing predictions of $E_{s h}$ vary and the experimental data are required to select the best microscopic approach. We noted that the
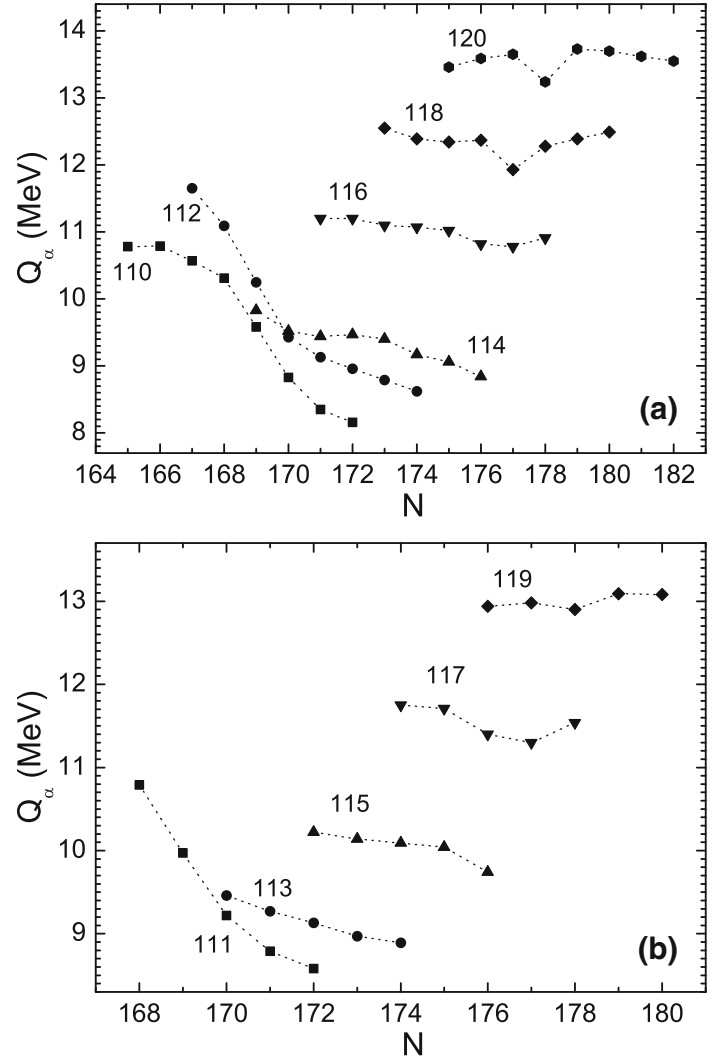

Fig. 21 The values of $\alpha$-decay energies (symbols connected by lines) calculated with the Mic-Mac model (FRDM2012) [230] for even- $Z$ (a) and odd- $Z$ (b) nuclei with $110 \leq Z \leq 120$

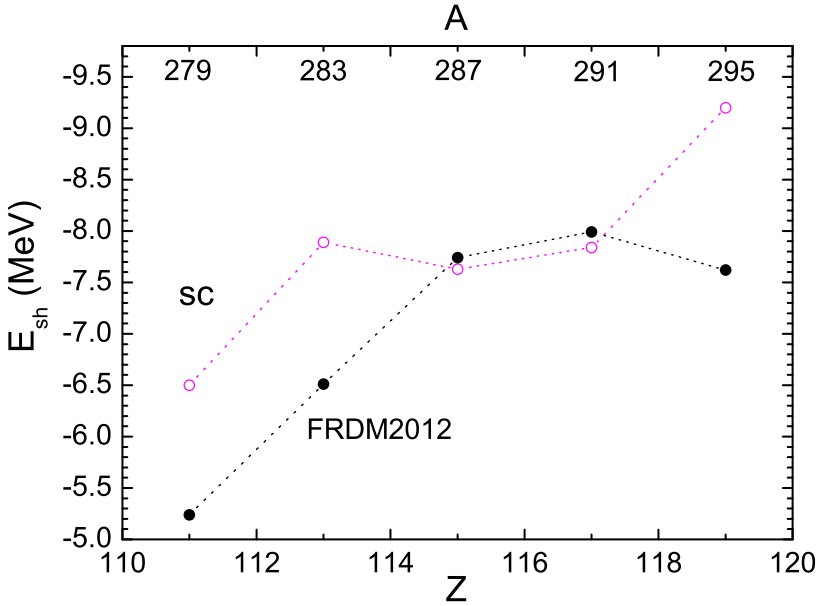

Fig. 22 The calculated ground-state shell corrections in the nuclei of $\alpha$-decay chain of ${ }^{295} 119$. The results are obtained with the MicMac method using the WS potential extracted from the self-consistent [sc] consideration (open circles). The microscopic corrections from the FRDM2012 [230] are shown by closed circles for comparison. The lines are drawn to guide the eye

predicted ground-state deformations are very close (within $10 \%$ ) in all approaches used. Different parameter sets used as input for the HFB calculations result typically in differ- 

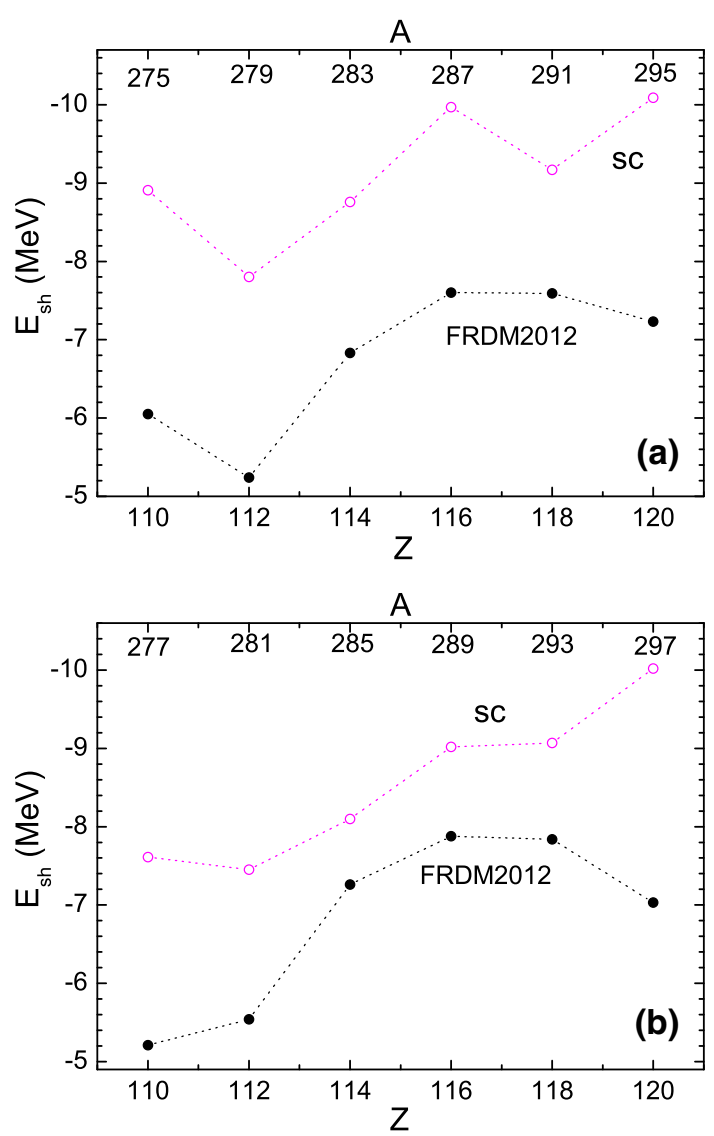

Fig. 23 The same as in Fig. 22, but for the nuclei of $\alpha$-decay chains of ${ }^{295,297} 120$

ent structures of heavy nuclei. So, the level order depends in some cases critically on the EDF underlying self-consistent approach. However, the shell closure at $Z=120$ is strongly supported in almost all self-consistent approaches while the neutron shell at $N=184$ depends on the model. We consider the nuclei for which the self-consistent approaches indicate stronger shell effects at $Z=120$ than at $Z=114$, opposite to the known Mic-Mac approaches [45,54-59,230]. Here, we show how to obtain similar results with the Mic-Mac model and relate it to the self-consistent models.

The quasiparticle energies are strongly affected by the single-particle levels and by the residual pairing and quasiparticle-phonon interactions. These interactions are used in core-polarization studies within the QPM [68,203, 253] where single-particle spectrum was obtained by the WS potential extracted from the HFB calculations. The quadrupole and octupole phonons and blocking effects in odd-even and even-odd nuclei are taken into account. In the region of the heaviest nuclei, the density of the singleparticle states near the Fermi level is rather high and the quasiparticle-phonon interaction tends to increase the admixtures of one-quasiparticle components because the QPM core-polarization effects induce couplings to three- quasiparticle configurations, described as quasiparticlephonon states. The QPM provides a quite realistic description of the spectral structure of correlated one-quasiparticle states, which is important for the analysis of the $\gamma$-transitions between these states. As a rule, the polarized low-lying quasiparticle states are typically dominated by a single component close to the unperturbed BCS eigenstate. However, the threeparticle admixtures affect the $\gamma$-transitions because they can occur through the admixture of components if the transition through the main component is hindered.

In Fig. 24, the calculated one-quasiproton spectra and $\alpha$ decays from the ground and possible isomeric states are shown in the nuclei of the $\alpha$-decay chain of the ${ }^{295} 119$ nucleus. Because the WS potential resulted from the HFB calculation is deeper than those in the phenomenological microscopic-macroscopic models [263,264], it results in a sparser one-quasiparticle spectrum. Moreover, the shell effects increase considerably at $Z=120$. The $\alpha$-decay chain of ${ }^{295} 119$ contains the elements with $Z \leq 115$ whose $\alpha$ decay energies are known experimentally [1]. The calculated and measured energies of $\alpha$-decays of ${ }^{287} \mathrm{Mc},{ }^{283} \mathrm{Nh}$, and ${ }^{279} \mathrm{Rg}$ are in a good agreement.

Because the parameters of single-particle potentials and spin-orbit interactions are different in Refs. [229,263,264], the order of one-quasiparticle levels is different as well. A remarkable feature is that the existence of low-lying isomeric state is predicted by all single-particle potentials considered. As seen in Fig. 24, in the evaporation residue ${ }^{295} 119$ the low-lying isomeric state $13 / 2^{+}$[606] can be populated with a probability close to the population probability of the ground state $1 / 2^{-}$[510]. In ${ }^{291} \mathrm{Ts}$, the state $1 / 2^{-}$[510] lies at 0.247 $\mathrm{MeV}$, but the state $13 / 2^{+}[606]$ is the ground state. Therefore, the possible $\alpha$-decay of ${ }^{295} 119$ from the isomeric $13 / 2^{+}$[606] and ground states easily occur into the corresponding states of ${ }^{291}$ Ts. So, two possible $\alpha$-decays of ${ }^{295} 119$ are expected to have different energies and half-lives. The difference of $Q_{\alpha}$ by $0.4 \mathrm{MeV}$ results in about one order of magnitude different half-lives. In comparison with the results of Ref. [229], the $\alpha$-decay of the ground state of ${ }^{295} 119$ occurs faster.

In ${ }^{291} \mathrm{Ts}$, both the ground $13 / 2^{+}[606]$ and the isomeric $3 / 2^{-}[511]$ states are populated in the $\alpha$-decay chain of ${ }^{295} 119$. The $\alpha$-decays from these states are hindered because in ${ }^{287} \mathrm{Mc}$ the $13 / 2^{+}[606]$ state is at about $0.5 \mathrm{MeV}$ and $3 / 2^{-}$[511] state has $E_{p q}>1 \mathrm{MeV}$. So, the future data on the $\alpha$-decay of ${ }^{291}$ Ts would be useful to conclude if the predictions of Ref. [229], where an unhindered $\alpha$-decay is expected, or the present predictions are reliable. The $\alpha$ decays of ${ }^{287} \mathrm{Mc},{ }^{283} \mathrm{Nh}$, and ${ }^{279} \mathrm{Rg}$ are unhindered. The shell corrections in ${ }^{295} 119$ and ${ }^{291}$ Ts are stronger than those for neighboring nuclei with $Z=120$ and 118 in Ref. [265] and their lifetimes with respect to fission are expected larger than 0.1 s. So, the $\alpha$-decay chain of ${ }^{295} 119$ is likely to be interrupted by fission below ${ }^{279} \mathrm{Rg}$. 
Fig. 24 Calculated energies of low-lying one-quasiproton states in the indicated nuclei of the $\alpha$-decay chain of ${ }^{295} 119$. The calculated values of $Q_{\alpha}$ are for the ground-state-ground-state $\alpha$-decay. The $\alpha$-decays with $\Delta K=0$ and $\Delta K \neq 0$ are traced by solid and dashed arrows, respectively. The experimental values of $Q_{\alpha}^{\exp }$ are from Ref. [1]

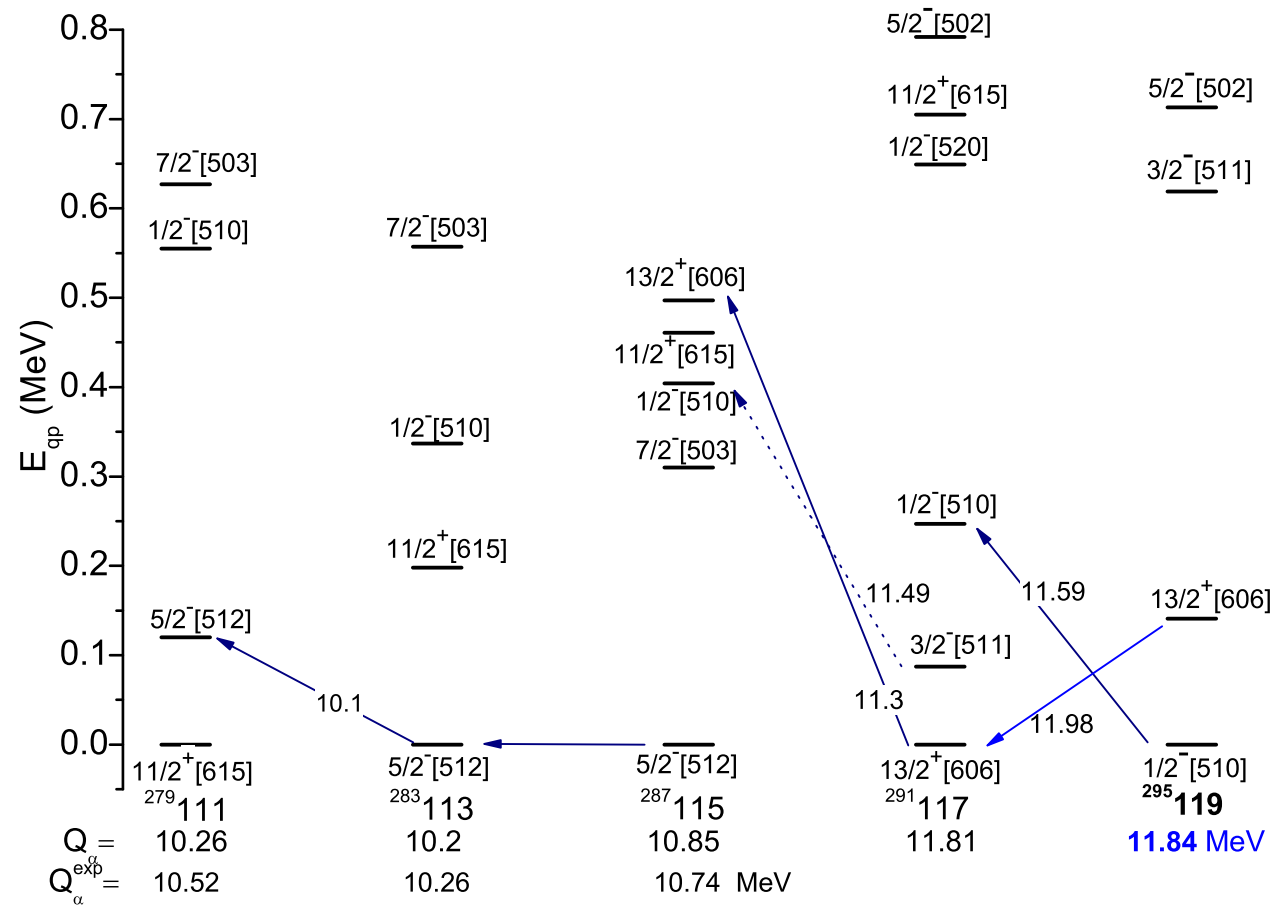

5.5 Fusion-evaporation reactions for the production of SHN with $Z=119$ and 120

The DNS fusion model [204-229] describes successfully fusion-evaporation reactions especially related to the production of SHN. In the DNS model, the fusion is considered as a diffusion in the mass asymmetry coordinate $\eta=\left(A_{1}-A_{2}\right) /\left(A_{1}+A_{2}\right)\left(A_{1}\right.$ and $A_{2}$ are the mass numbers of the DNS nuclei). The evaporation residue cross-section in the $x n$ evaporation channel is determined as

$$
\begin{aligned}
\sigma_{E R}^{x n}\left(E_{\mathrm{c} . \mathrm{m} .}\right)= & \sum_{J} \sigma_{c}\left(E_{\mathrm{c} . \mathrm{m} .}, J\right) P_{C N}\left(E_{\mathrm{c} . \mathrm{m} .}, J\right) \\
& \times W_{\text {sur }}^{x n}\left(E_{\mathrm{c} . \mathrm{m} .}, J\right)
\end{aligned}
$$

The capture cross-section $\sigma_{c}\left(E_{\mathrm{c} . \mathrm{m}}, J\right)$ defines the transition of the colliding nuclei through the Coulomb barrier and the formation of the DNS when the kinetic energy above the barrier is transformed into the excitation energy of the DNS and the angular momentum $J$ of the relative motion is redistributed in the DNS. For the reactions considered here, the maximum evaporation residue cross-sections occur at energies $E_{\text {c.m. }}$ at which all orientations of deformed nuclei contribute to the fusion. Therefore, in our case there is no noticeable loss of the cross-section during the capture.

The probability of complete fusion $P_{\mathrm{CN}}\left(E_{\mathrm{c} . \mathrm{m} .}, J\right)$ depends on the competition between the complete fusion and quasifission after the capture stage. This competition can strongly reduce the value of $\sigma_{\mathrm{ER}}^{\mathrm{xn}}\left(E_{\mathrm{c} . \mathrm{m}}\right)$. The survival probability $W_{\text {sur }}^{\mathrm{xn}}$ takes into consideration the cooling-down of the compound nucleus by emission of $x$ neutrons in the competition with fission. The detailed description of the calculations of $\sigma_{c}, P_{\mathrm{CN}}$, 
Fig. 25 The same as in Fig. 24, but for the nuclei of $\alpha$-decay chain of ${ }^{295} 120$
Fig. 26 The same as in Fig. 24, but for the nuclei of $\alpha$-decay chain of ${ }^{297} 120$

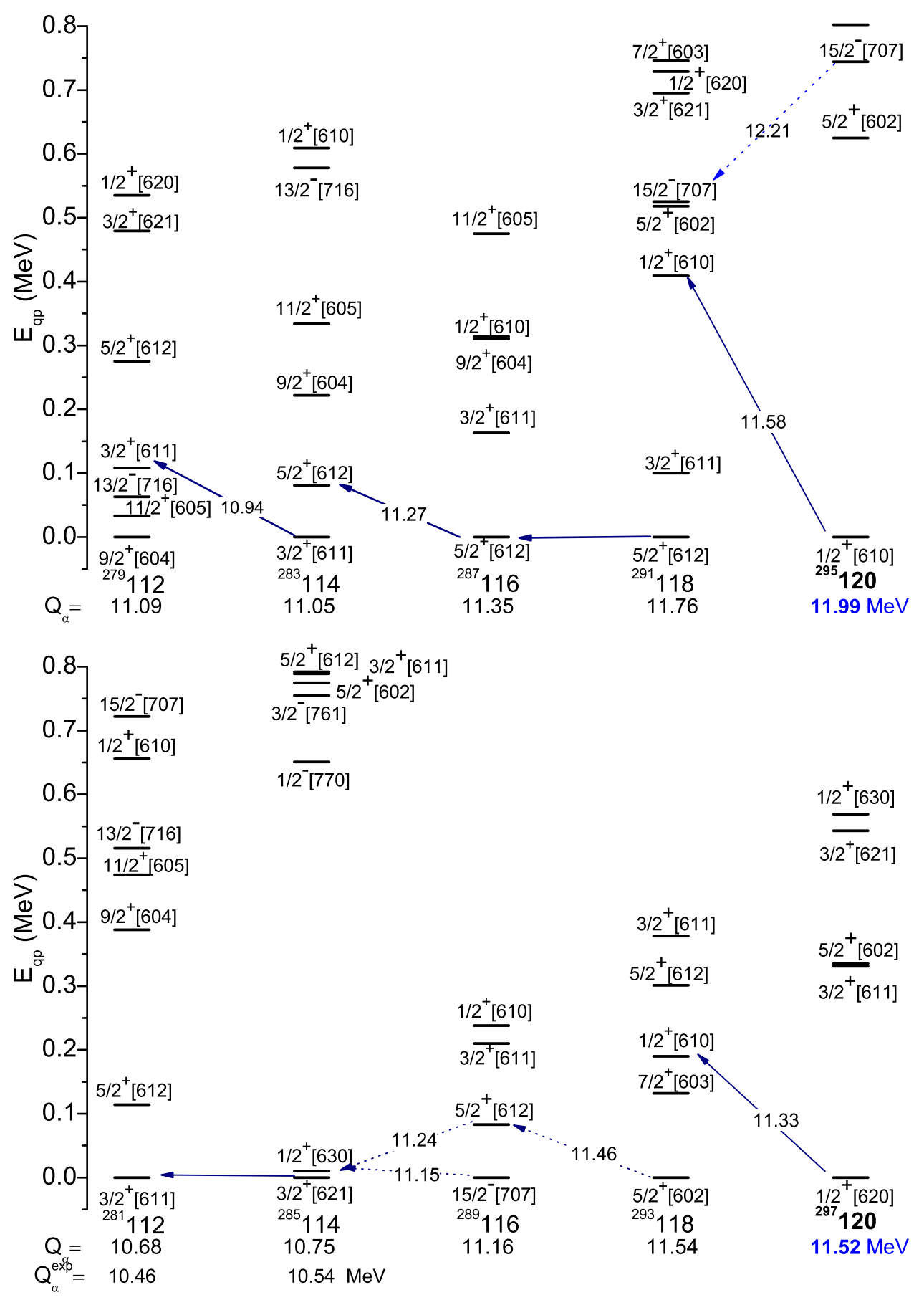

and $W_{\text {sur }}^{\mathrm{xn}}$ is found in Ref. [205]. As estimated, the uncertainty of our calculated cross-sections is within a factor of 2-4.

\subsection{1 $B_{f}-B_{n}$ in $S H N$}

The survival probability of compound nucleus strongly depends on the difference $B_{f}-B_{n}$, where $B_{f}$ and $B_{n}$ are the height of fission barrier and the neutron separation energy, respectively. The value of $B_{f}$ is mainly determined by the amplitude of the shell correction $E_{\text {sh }}$ in the ground state of nuclei considered. As a result, the shell effects prevent the fission of SHN and the value of $B_{f} \approx-E_{\text {sh }}$ strongly depends on the neutron and proton numbers of the compound nucleus, especially, on how close they are to the magic numbers. At fixed charge number the predicted values of $B_{n}$ steadily decrease in the region of $N \geq 170$ with increasing $N$. The values of $B_{n}$ predicted with different models vary within 0.5 $\mathrm{MeV}$ and the shell effects cause the difference in the $B_{f}-B_{n}$ calculated (Figs. 27, 28).

As seen in Fig. 28, the Mic-Mac model [230] predicts a closed proton shell at $Z=114$ and the fission barrier grows with $N$ up to $N=178-180$. At fixed neutron number 

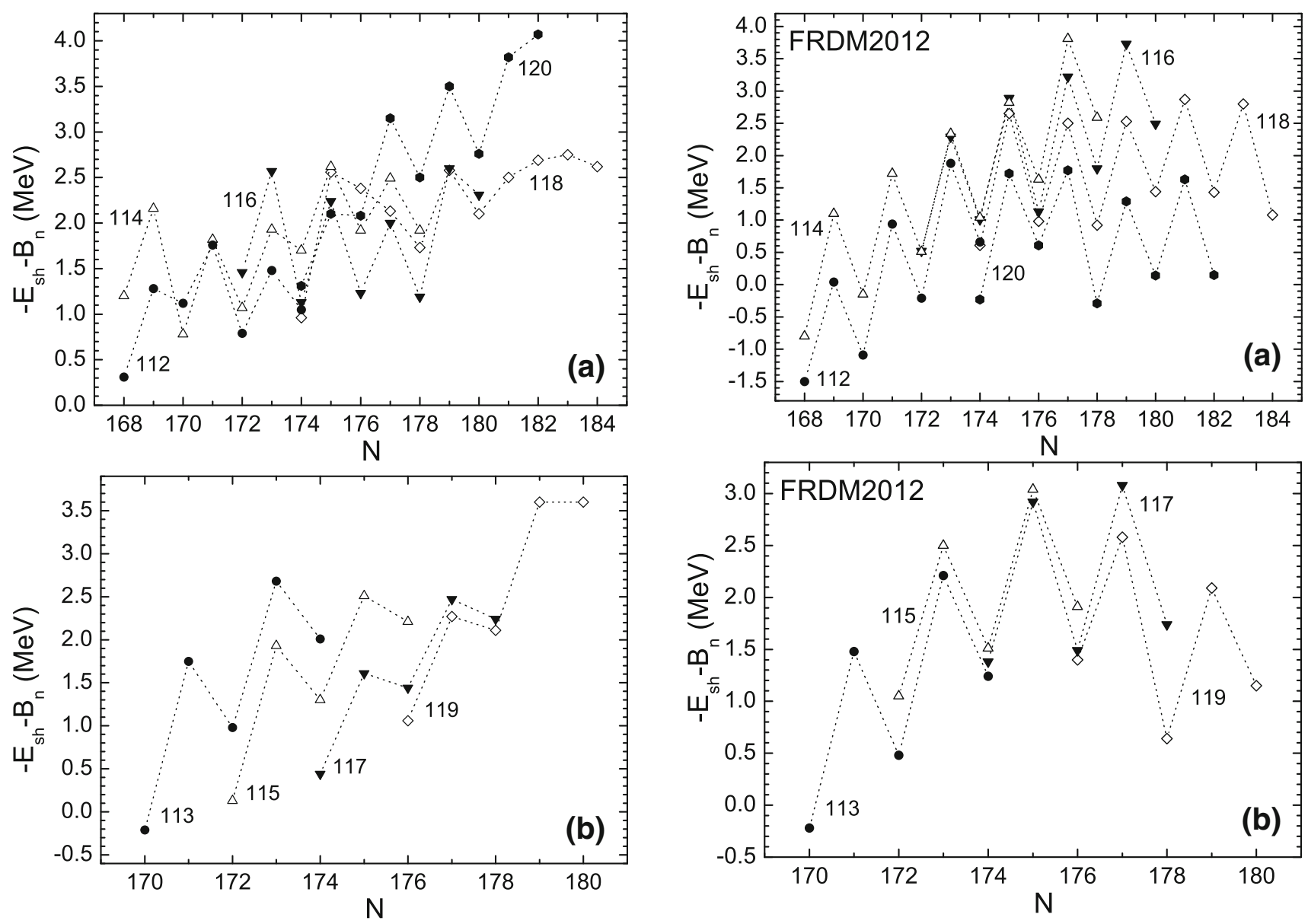

Fig. 27 The isotopic dependence of the value of $B_{f}-B_{n}$. The results are obtained with the Mic-Mac method using the WS potential extracted from the self-consistent consideration. The fission barrier $B_{f}$ is assumed to have the absolute value of the shell correction at the ground state of the nucleus. The results for the isotopes related to the indicated even- $Z$ (a) and odd- $Z$ (b) cases are shown by symbols connected by lines

and $Z>114$, the height of the fission barrier decreases with increasing $Z$. The results in Fig. 27 also indicate the expected shell closure at $Z=114$. However, the shell effects at $Z=120$ are even stronger. In Fig. 27, the fission barrier increases when $N$ approaches $N=184$. The nuclei with $Z=120$ and $N=180-182$, where the fission barriers are rather high, are expected to be the most stable nuclei beyond those with $Z=114$ and $N=176-178$. Note that the shell closure at $Z=120$ have been obtained also by the RMF model calculations, e.g., Ref. [131].

\subsubsection{Fusion $Q$-values}

For the complete fusion reactions ${ }^{48} \mathrm{Ca},{ }^{50} \mathrm{Ti}+{ }^{238} \mathrm{U},{ }^{244} \mathrm{Pu}$, ${ }^{248} \mathrm{Cm},{ }^{249} \mathrm{Cf},{ }^{54} \mathrm{Cr}+{ }^{238} \mathrm{U},{ }^{244} \mathrm{Pu},{ }^{248} \mathrm{Cm}$, and ${ }^{58} \mathrm{Fe}+{ }^{238} \mathrm{U},{ }^{244} \mathrm{Pu}$, we present the $Q$-values (Fig. 29) calculated with different predictions of binding energies of compound nucleus. One can see that the results [curves sc] are within the existing

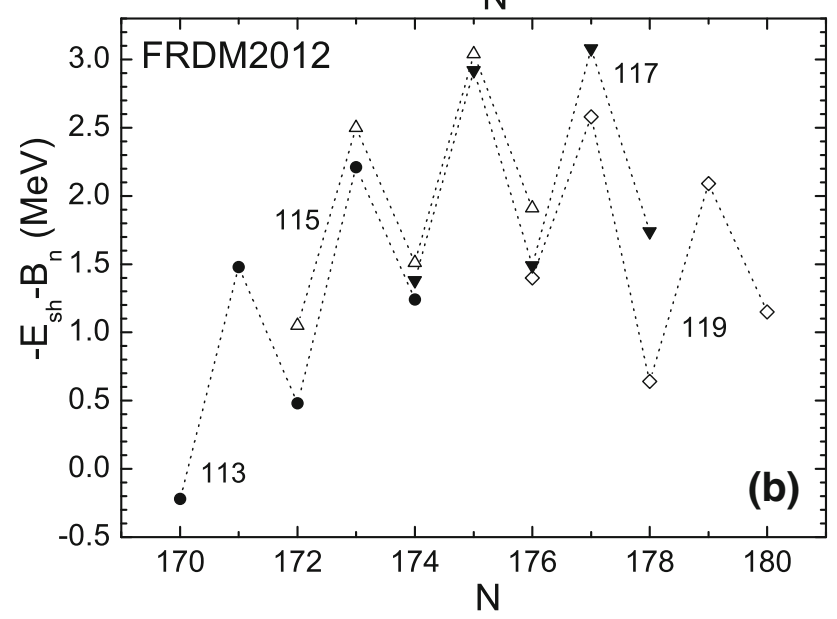

Fig. 28 The same as in Fig. 27, but for the nuclei from the FRDM2012 mass table [230]

uncertainty of 5-7 MeV provided by previous calculations. For smaller $Z$, they are close to the results obtained with the data of Refs. [266-269]. For larger $Z$, our calculated $Q$-values approach those obtained with the Mic-Mac model [230]. So, the uncertainty in the $Q$-value leads to an uncertainty in excitation energy of compound nuclei in the range of $5-7 \mathrm{MeV}$ which, in fact, is close to the neutron separation energy.

\subsubsection{Predictions of evaporation residue cross-sections}

Using the predicted $M_{\mathrm{th}}, E_{\mathrm{sh}}$, and $B_{n}$, we calculated the evaporation residue cross-sections in the reactions ${ }^{48} \mathrm{Ca}$, ${ }^{50} \mathrm{Ti}+{ }^{238} \mathrm{U},{ }^{244} \mathrm{Pu},{ }^{248} \mathrm{Cm},{ }^{249} \mathrm{Cf},{ }^{54} \mathrm{Cr}+{ }^{238} \mathrm{U},{ }^{244} \mathrm{Pu},{ }^{248} \mathrm{Cm}$, ${ }^{58} \mathrm{Fe}+{ }^{238} \mathrm{U},{ }^{244} \mathrm{Pu}$, and ${ }^{64} \mathrm{Fe}+{ }^{238} \mathrm{U}$ (Fig. 30). In the reactions ${ }^{48} \mathrm{Ca}+{ }^{238} \mathrm{U},{ }^{248} \mathrm{Cm},{ }^{249} \mathrm{Cf}$ the experimental values of $\sigma_{\mathrm{ER}}^{3 n}$ are $0.5-2.5 \mathrm{pb}$, about $1 \mathrm{pb}$, and $0.5 \mathrm{pb}$ [1], respectively. Thus, the difference between the calculated and experimental $\sigma_{\mathrm{ER}}$ are within the experimental and theoretical uncertainties. The good description of an existing data indicates reliability of the predictions for the reactions with projectiles heavier than 

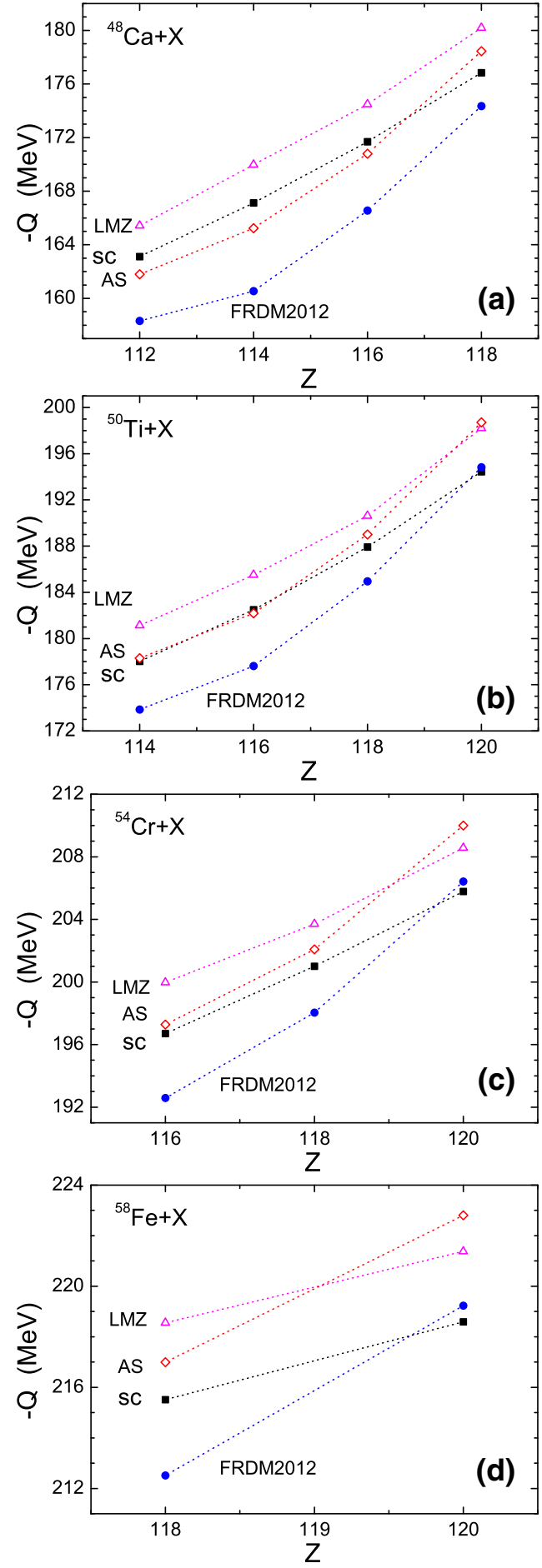

Fig. 29 The $Q$-values for complete fusion calculated in the indicated reactions ${ }^{48} \mathrm{Ca},{ }^{50} \mathrm{Ti}+{ }^{238} \mathrm{U},{ }^{244} \mathrm{Pu},{ }^{248} \mathrm{Cm},{ }^{249} \mathrm{Cf}$, ${ }^{54} \mathrm{Cr}+{ }^{238} \mathrm{U},{ }^{244} \mathrm{Pu},{ }^{248} \mathrm{Cm}$, and ${ }^{58} \mathrm{Fe}+{ }^{238} \mathrm{U},{ }^{244} \mathrm{Pu}$, with the mass excesses of compound nuclei $Z$ from the the Mic-Mac method with the WS potential extracted from the self-consistent consideration (sc, solid squares), Refs. [230] (FRDM2012, solid circles), Refs. [263,264] (AS, open diamonds), and Refs. [266-269] (LMZ, open triangles)

${ }^{48} \mathrm{Ca}$. In comparison to our previous calculations [204] with the mass table of Ref. [45], the values of $\sigma_{\mathrm{ER}}$ decreases slower with increasing $Z$. The stronger shell effects revealed here

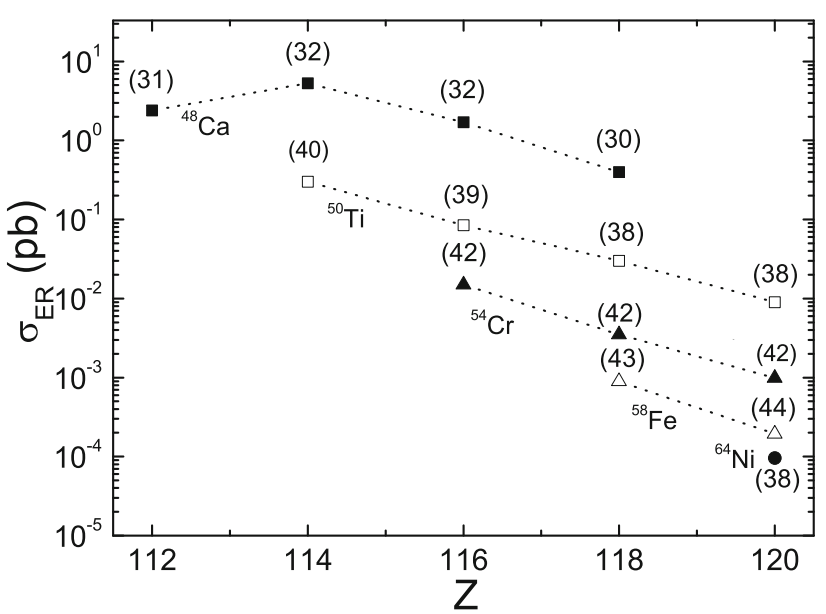

Fig. 30 The evaporation residue cross-sections in the maxima of excitation functions versus charge number $Z$ for the reactions ${ }^{48} \mathrm{Ca},{ }^{50} \mathrm{Ti}+{ }^{238} \mathrm{U},{ }^{244} \mathrm{Pu},{ }^{248} \mathrm{Cm},{ }^{249} \mathrm{Cf},{ }^{54} \mathrm{Cr}+{ }^{238} \mathrm{U},{ }^{244} \mathrm{Pu},{ }^{248} \mathrm{Cm},{ }^{58} \mathrm{Fe}+$ ${ }^{238} \mathrm{U},{ }^{244} \mathrm{Pu}$, and ${ }^{64} \mathrm{Ni}+{ }^{238} \mathrm{U}$. The predicted properties of $\mathrm{SHN}$ are used from the Mic-Mac method with the WS potential extracted from the self-consistent consideration. The excitation energies in $\mathrm{MeV}$ of compound nuclei are given in brackets

for nuclei with $Z>118$ result in larger survival probabilities and larger values of $\sigma_{\mathrm{ER}}$.

With ${ }^{50} \mathrm{Ti}$ beam the values of $\sigma_{\mathrm{ER}}$ for the nuclei with $Z=$ $114-118$ are expected to be $\sim 10$ times smaller than those with ${ }^{48} \mathrm{Ca}$ beam (Fig. 30). The main reason for this is the decrease of $P_{\mathrm{CN}}$ in Eq. (89) with mass asymmetry in the entrance channel of reaction. With ${ }^{50} \mathrm{Ti}$ the nucleus ${ }^{295} 120$ is predicted to be produced with the maximum cross-section of $\sim 8 \mathrm{fb}$. For the production of nucleus with $Z=120$, the beams ${ }^{54} \mathrm{Cr},{ }^{58} \mathrm{Fe}$, and ${ }^{64} \mathrm{Ni}$ would lead to smaller crosssections. For example, in the ${ }^{54} \mathrm{Cr}+{ }^{248} \mathrm{Cm}$ reaction, $\sigma_{\mathrm{ER}} \approx 1$ $\mathrm{fb}$.

We calculated the evaporation residue cross-sections in the reactions ${ }^{50} \mathrm{Ti}+{ }^{A} \mathrm{Cf}(A=248-251)$ leading to the compound nuclei with $Z=120$ (Fig. 31). As in Refs. [225-227], the value of $\sigma_{E R}$ changes within factor 2-4 in the treated interval of mass numbers $A$. The values of $\sigma_{\mathrm{ER}}$ are almost the same (about $15 \mathrm{fb}$ ) in the cases of ${ }^{250} \mathrm{Cf}$ and ${ }^{251} \mathrm{Cf}$ targets. In the case of ${ }^{249} \mathrm{Cf}$ target and ${ }^{50} \mathrm{Ti}$ beam, the value of $\sigma_{\mathrm{ER}}$ is smaller than those in the reactions ${ }^{50} \mathrm{Ti}+{ }^{250,251} \mathrm{Cf}$, about 8 $\mathrm{fb}$. For the production of nuclei with $Z=120$, the reactions with ${ }^{50} \mathrm{Ti}$ are favorable over those with ${ }^{48} \mathrm{Ti}$. The optimal excitation energies in the reactions with ${ }^{50} \mathrm{Ti}$ are about $4-7$ $\mathrm{MeV}$ smaller. As a result, the survival probabilities and, correspondingly, the production cross-sections are larger in the ${ }^{50} \mathrm{Ti}$ reactions. Note that for the same reason the difference is relatively small between the cross-sections in the reactions ${ }^{58} \mathrm{Fe}+{ }^{244} \mathrm{Pu}$ and ${ }^{64} \mathrm{Ni}+{ }^{238} \mathrm{U}$ (Fig. 30).

In the reactions ${ }^{54} \mathrm{Cr}+{ }^{A} \mathrm{Cm} \rightarrow 120$, the value of $\sigma_{\mathrm{ER}}$ decreases by factor of about two with the mass number of target-nucleus from $A=248$ to $A=245$ (Fig. 32). In the reactions ${ }^{50} \mathrm{Ti}+{ }^{249} \mathrm{Bk}$, the nucleus 119 is predicted to be produced 

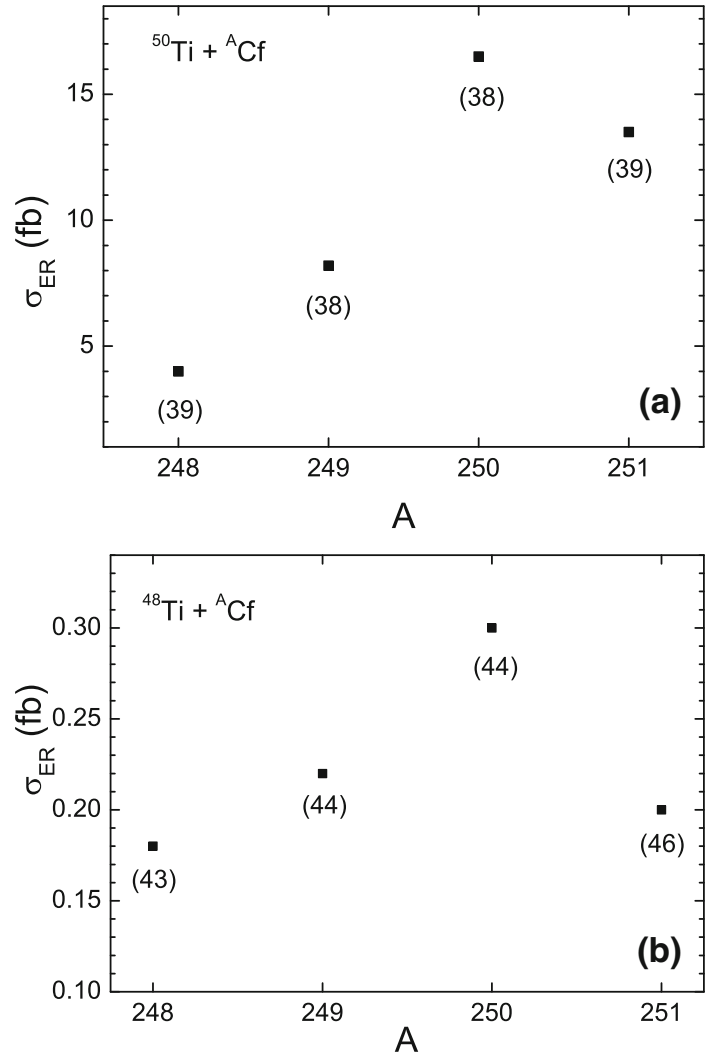

Fig. 31 The same as in Fig. 30, but for the reactions ${ }^{50} \mathrm{Ti}+{ }^{A} \mathrm{Cf}$ (a) and ${ }^{48} \mathrm{Ti}+{ }^{A} \mathrm{Cf}(\mathbf{b})$

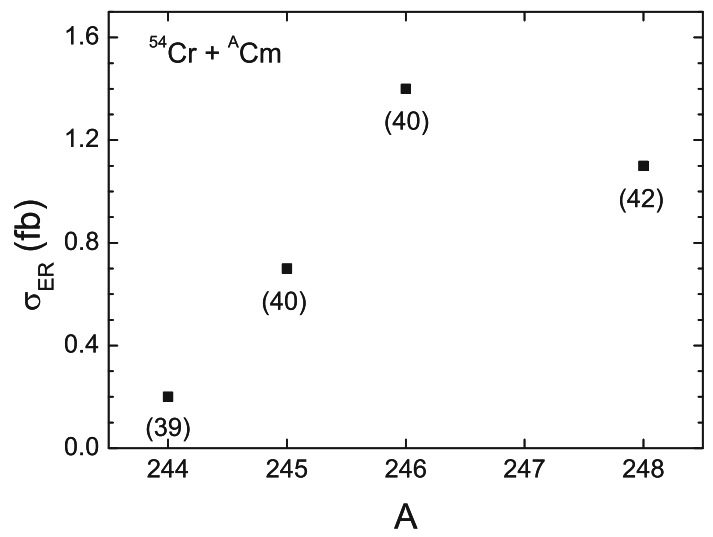

Fig. 32 The same as in Fig. 30, but for the reactions ${ }^{54} \mathrm{Cr}+{ }^{A} \mathrm{Cm}$

with the maximum cross-section $19 \mathrm{fb}$ (Fig. 33). The increase of $\sigma_{\mathrm{ER}}$ with $A$ is mostly due to the increase of the survival probability. In the $4 n$ evaporation channels of the reactions ${ }^{50} \mathrm{Ti}+{ }^{235,236,238} \mathrm{U}$, the predicted maximum production crosssections are about $0.2-0.5 \mathrm{pb}$ (Fig. 34). These reactions can be measured and compared with the ${ }^{48} \mathrm{Ca}$-induced reactions.

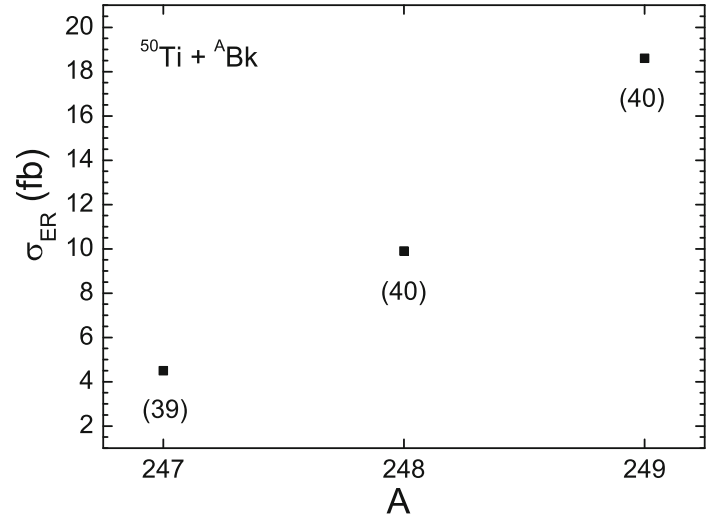

Fig. 33 The same as in Fig. 30, but for the reactions ${ }^{50} \mathrm{Ti}+{ }^{A} \mathrm{Bk}$

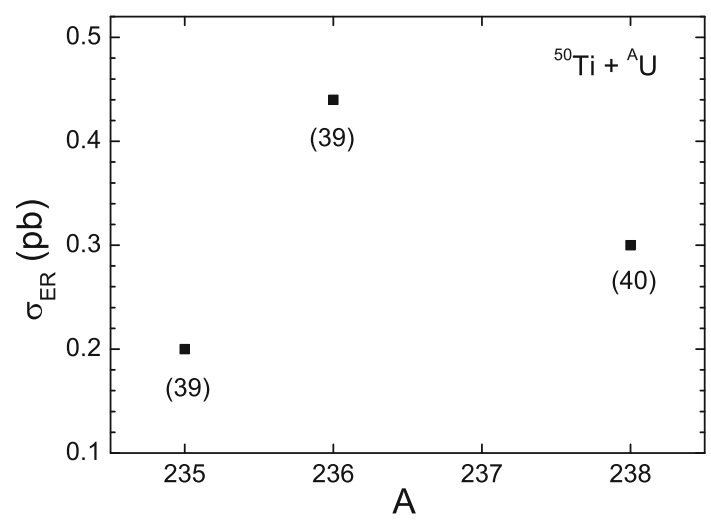

Fig. 34 The same as in Fig. 31, but for the reactions ${ }^{50} \mathrm{Ti}+{ }^{A} \mathrm{U}$

\section{Isotopic trends of the nuclear surface properties of spherical nuclei}

Average central densities, nuclear radius, and surface diffuseness are basic quantities characterizing finite nuclear systems. Extensive discussions are currently made on the proton-neutron differences of proton-neutron nuclear radii and diffusenesses to study the symmetry energy [270-276]. The polarization of the nuclear surface in deformed nuclei was also considered in Refs. [277,278]. While the central density and radius are mainly related to the internal $N N$ forces, or to the properties of nuclear matter, the diffuseness of the nuclear surface turns out to be related to the external part of these forces, being the effect of finite Fermi systems. The nuclear surface region is of particular importance for nuclear reactions. From the point of view of nuclear dynamics, this is a transitional region where the in-medium interactions gradually undergo into the free $N N$ interactions. The TFFS [171], for example, accounts for these transient effects explicitly introducing internal and external interactions. 
6.1 EDF methods to nuclear ground-state density distributions

\subsubsection{Method I. GEDF}

Nuclear binding energies, single-particle states, and groundstate densities are described by the GEDF

$$
\begin{aligned}
\mathcal{E}(\rho, \tau, \kappa)= & \mathcal{E}_{\text {kin }}(\tau)+\frac{1}{2} \mathcal{E}_{\text {int }}(\rho)+\frac{1}{2} \mathcal{E}_{\text {pair }}(\rho, \kappa) \\
& -\sum_{q} \lambda_{q} \rho_{q},
\end{aligned}
$$

given by the kinetic-energy density $\mathcal{E}_{\text {kin }}$, the interaction energy density $\mathcal{E}_{\text {int }}$ and the pairing interaction density $\mathcal{E}_{\text {pair }}$. In order to enforce particle number conservation, the proton and neutron chemical potentials $\lambda_{q}$ are introduced. The interactions contained in $\mathcal{E}_{\text {int }}$ and $\mathcal{E}_{\text {pair }}$ are derived from inmedium $G$-matrix interactions which is supplemented by the effective three-body interaction in order to describe properly the saturation properties of infinite nuclear matter. So, $\mathcal{E}_{\text {int }}$ contains the Coulomb, spin-orbit, and residual interactions.

Variations of $\mathcal{E}$ lead to Eq. (12) for the quasiparticle wave functions $u_{q \alpha}$ and $v_{q \alpha}$. For the present applications, they are solved in the BCS approximation with state-dependent gaps, as discussed before. Solving these equations with the parameters defined in Ref. [112], we find the nucleon-density profile. The equations are solved by direct numerical integration using the Numerov-Cowell method (see, e.g., Ref. [279]) which provides a stable and fast algorithm optimized for the solution of ordinary second order differential equations. In practice, the eigenvalue problem is solved by matching outward and inward integrated solutions by variation of $e_{q \alpha}$ until both solutions and their first derivatives are smoothly connected. In comparison to the widely used expansion in terms of oscillator wave functions, our approach avoids artificial cut-off effects and guarantees the correct exponential fall-off of bound state wave functions and densities in the asymptotic region up to arbitrarily large radii. Also particle states will be described with the proper asymptotic oscillatory behavior.

The EDF approach leads to a good description of nuclear binding energies (Fig. 35). The experimentally available data [200] are well reproduced. The slight deviations around ${ }^{56} \mathrm{Ni}$ are most likely due to the neglection of the rank-2 tensor interactions which have been found to add a non-negligible amount of energy to the total binding energies around subshell closures in even-even nuclei in this mass region [280]. Further results on the ground-state properties and nuclear excitations of beta-stable and exotic nuclei were presented in Refs. [112,199,281]. Here, in Fig. 36 we demonstrate a good description of the measured [282] charge density distribution for ${ }^{58,64} \mathrm{Ni}$. As seen, the quality of description of the characteristics of proton density distribution is similar to those in Refs. [151,273-276].

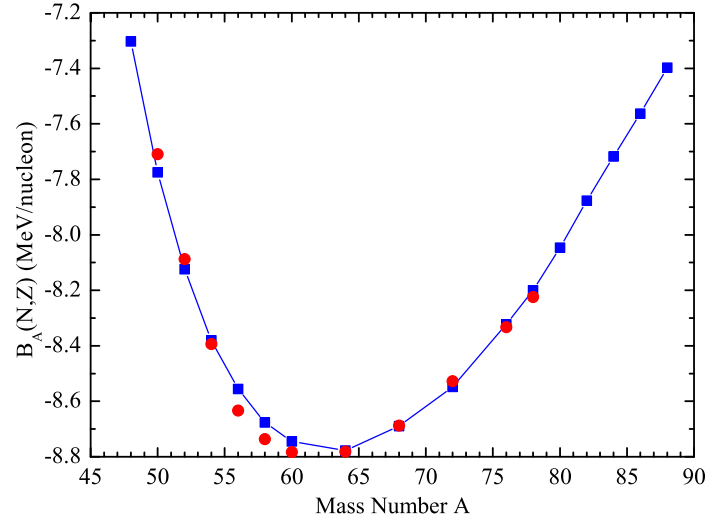

Fig. 35 The binding energies per nucleon for the Ni isotopic chain. The energy-density functional results (solid squares connected by lines) are compared with the experimental data (solid circles) of Ref. [200]. The data are reproduced with an average root-mean deviation per item of $B_{A}=134.08 \mathrm{keV}$

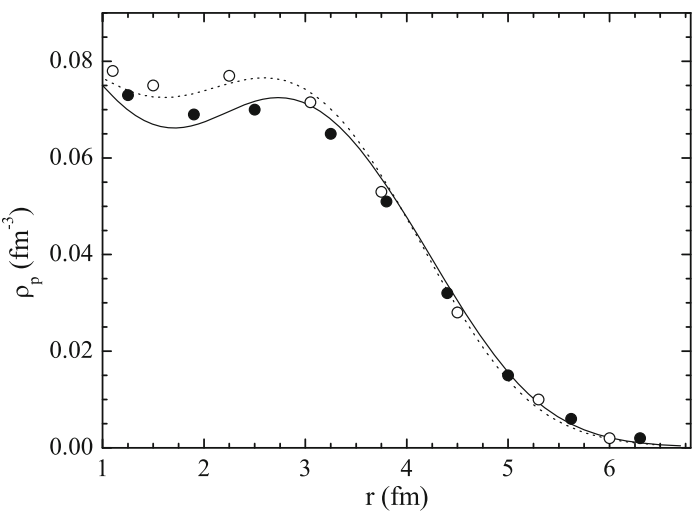

Fig. 36 The calculated radial distributions of the proton density for ${ }^{58} \mathrm{Ni}$ (dotted line) and ${ }^{64} \mathrm{Ni}$ (solid line) are compared with the experimental data [282] shown by open and solid symbols, respectively

\subsubsection{Method II. Phenomenological Fayans EDF}

Earlier in this review, the success and the shortcomings of the widely used SkEDFs have been mentioned repeatedly. The reason behind the overwhelming popularity of the Skyrmemodel is the comparatively simple and transparent structure of the EDF. Among the many attempts to derive improved SkEDFs, Fayans has formulated a particularly successful and promising approach. Over the years, several versions of the Fayans EDF (FEDF) have been developed, among those the FaNDF $^{0}$ [283-285], the DF3-version [146,286,287], and the DF3-a functional [288] are being used. The SkEDFs are given essentially by polynomials of densities and spin current densities, derivatives thereof, and anomalous densities, supplemented by terms of rational powers of the densities mimicking the intrinsic density dependencies of interactions and many-nucleon forces. The FEDFs are of a more complex structure by allowing also rational functionals on particle densities and derivatives. Such structures are actually used in 
the GEDF and the covariant DDRH vertex functionals. While in the latter two cases the rational functionals were derived from $G$-matrix interactions, Fayans came independently to the same conclusions on completely empirical grounds.

The Fayans kinetic-energy functional is of standard form $\sim\left|\nabla \psi_{\mathrm{sq}}\right|^{2}$. Different from most other EDF models, the FEDF is constructed such that the effective proton and neutron masses are given by the free nucleon masses, i.e. they are constant. This establishes a link to the theory of finite Fermi systems [174]. Thus, in the Fayans approach the kineticenergy operator is of the form obtained before for the reduced Schrödinger-wave equation. The interaction part of the FEDF is composed of volume, surface, spin-orbit, Coulomb, and pairing components, respectively,

$\mathcal{E}_{\text {int }}^{(\mathrm{Fy})}=\mathcal{E}_{\mathrm{v}}^{(\mathrm{Fy})}+\mathcal{E}_{\mathrm{s}}^{(\mathrm{Fy})}+\mathcal{E}_{1 \mathrm{~s}}^{(\mathrm{Fy})}+\mathcal{E}_{\mathrm{C}}^{(\mathrm{Fy})}+\mathcal{E}_{\mathrm{P}}^{(\mathrm{Fy})}$,

where different from Eqs. (1) and (90) the factors $\frac{1}{2}$ are absorbed into the functionals. Density dependencies are introduced in terms of dimensionless isoscalar $(I=+)$ and isovector $(I=-)$ normalized density coefficients,

$x_{I}=\frac{\rho_{I}}{\rho_{D}}, \quad x_{\text {pair }}=\frac{\rho_{+}}{\rho_{P}}$,

where $\rho_{D}$ and $\rho_{P}$ are used as numerical scaling parameters which are introduced for convenience only. In practice, however, the values of these two parameters are chosen as the saturation density of nuclear matter, $\rho_{D} \sim \rho_{P} \sim \rho_{\text {sat }}=0.16$ $\mathrm{fm}^{-3}$. With these notations, the volume term $\mathcal{E}_{\mathrm{v}}^{(\mathrm{Fy})}$ is defined by the rational functional:

$\mathcal{E}_{\mathrm{v}}^{(\mathrm{Fy})}=C_{0} \rho_{\mathrm{D}}^{2}\left[a_{+}^{\mathrm{v}} \frac{1-h_{1+}^{\mathrm{v}} x_{+}^{\sigma}}{1+h_{2+}^{\mathrm{v}} x_{+}^{\sigma}} x_{+}^{2}+a_{-}^{\mathrm{v}} \frac{1-h_{1-}^{\mathrm{v}} x_{+}}{1+h_{2-}^{\mathrm{v}} x_{+}} x_{-}^{2}\right]$,

resembling a Padé approximant. In a later work, the same ansatz was used by Erler et al. in the Skyrme context [289]. The constant $C_{0}$, in units of $\mathrm{MeV} \mathrm{fm}^{3}$, serves as overall normalization factor and is given by the inverse of the TFFS density of states $C_{0}=C^{-1}\left(k_{F}\left(\rho_{D}\right)\right)$; see Eq. (20). An optimal description of the observables is achieved for $\sigma=1 / 3$.

The important new aspect of the FEDF is the surface term, given also in rational form:

$\mathcal{E}_{\mathrm{s}}^{(\mathrm{Fy})}=C_{0} \rho_{\mathrm{D}}^{2} \frac{a_{+}^{\mathrm{s}} r_{s}^{2}\left(\nabla x_{+}\right)^{2}}{1+h_{+}^{\mathrm{s}} x_{+}^{\sigma}+h_{\nabla}^{\mathrm{s}} r_{s}^{2}\left(\nabla x_{+}\right)^{2}}$.

Attention should be paid again to the novel fractional form of the density-dependent couplings and especially the gradient terms in the denominator.

The spin-orbit functional is derived from zero-range twobody spin-orbit and tensor interactions; see, e.g., [147]. In a spin-saturated spherical nucleus, one finds

$\mathcal{E}_{\mathrm{ls}}^{(\mathrm{Fy})}=C_{0} \rho_{D}^{2}\left(a_{l s} \rho_{+} \nabla \cdot \mathbf{J}_{+}+a_{l s}^{\prime} \rho_{-} \nabla \cdot \mathbf{J}_{-}+g \mathbf{J}_{+}^{2}+g^{\prime} \mathbf{J}_{-}^{2}\right)$.

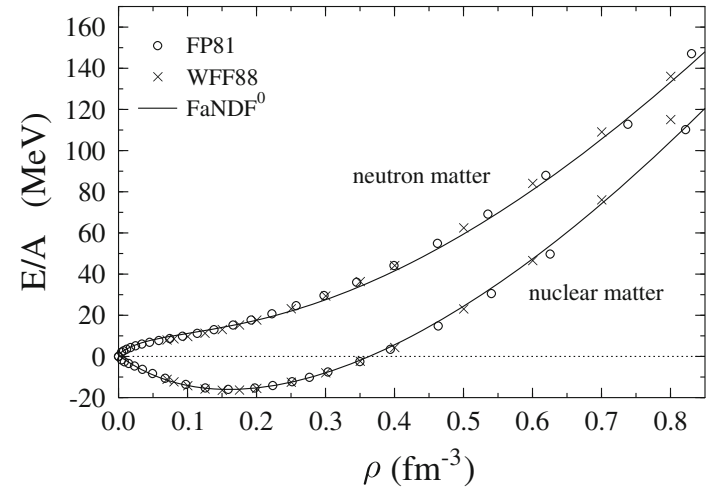

Fig. 37 The energy per nucleon versus density in nuclear and neutron matter, used to fix the parameters of the FEDF volume part. The equations of state obtained with the FaNDF ${ }^{0}$ EDF (lines). Open circles and crosses are the results by Friedman and Pandharipande [290] (FP81) and Wiringa et al. [291] (WFF88), respectively (from Ref. [292])

The isoscalar and isovector spin currents are denoted by $\mathbf{J}_{+,-}$, supplemented by the (rarely used) quadratic tensor terms. Expressions for $\mathbf{J}_{+,-}$are found elsewhere; see, e.g., [77]. The same kind of structure is encountered in the SkEDFs, typically with $g=g^{\prime}=0$.

In a finite nucleus, the FEDF Coulomb part is defined similar to Eq. (43) including the factor of $\frac{1}{2}$,

$\mathcal{E}_{C}^{(\mathrm{Fy})}=E_{H}^{(\mathrm{Fy})}+\mathcal{E}_{\mathrm{C}, \mathrm{ex}}^{(\mathrm{Fy})}$

but with an exchange correction term modified by the additional Coulomb-nuclear correlation term $\sim h_{\mathrm{C}}$ :

$\mathcal{E}_{\mathrm{C}, \mathrm{ex}}^{(F y)}=-\frac{3}{4}\left(\frac{3}{\pi}\right)^{1 / 3} e^{2} \rho_{\mathrm{ch}}^{4 / 3}\left(1-h_{\mathrm{C}} x_{+}^{\sigma}\right)$.

The FEDF pairing functional is of a peculiar structure:

$\mathcal{E}_{\mathrm{Fy}}^{\mathrm{P}}=C_{0} \sum_{q} \kappa_{q}^{*}\left[f_{\mathrm{ex}}^{\xi}+h_{+}^{\xi} x_{\text {pair }}^{\gamma}+f_{\nabla}^{\xi} r_{s}^{2}\left(\nabla x_{\text {pair }}\right)^{2}\right] \kappa_{q}$,

constructed so as to account effectively for polarization effects from the coupling to vibrational modes of the nuclear core as described by the novel density-gradient term. Closer inspection reveals that the term in brackets corresponds to the particle-particle pairing interaction $G_{\text {pair }}$, used in the GEDF approach, see Eqs. (36) and (39), where in the latter case the momentum dependence was replaced by averaging over the Fermi sphere.

The first two terms of Eq. (98) are a common feature of TFFS [171] and modern SkHFB EDFs [125]. The gradient term, introduced in [283], turned out to be of principal importance for reproducing the odd-even staggering effect in charge radii of long isotopic chains, e.g., $\mathrm{Ca}, \mathrm{Sn}$, and $\mathrm{Pb}$. This term is also important for accounting for negative isotopic shifts [146]. In Ref. [146], FEDF parameter set DF3 was used with parameters fitted to the equation of state of nuclear and neutron matter by Friedman-Pandharipande [290] and 

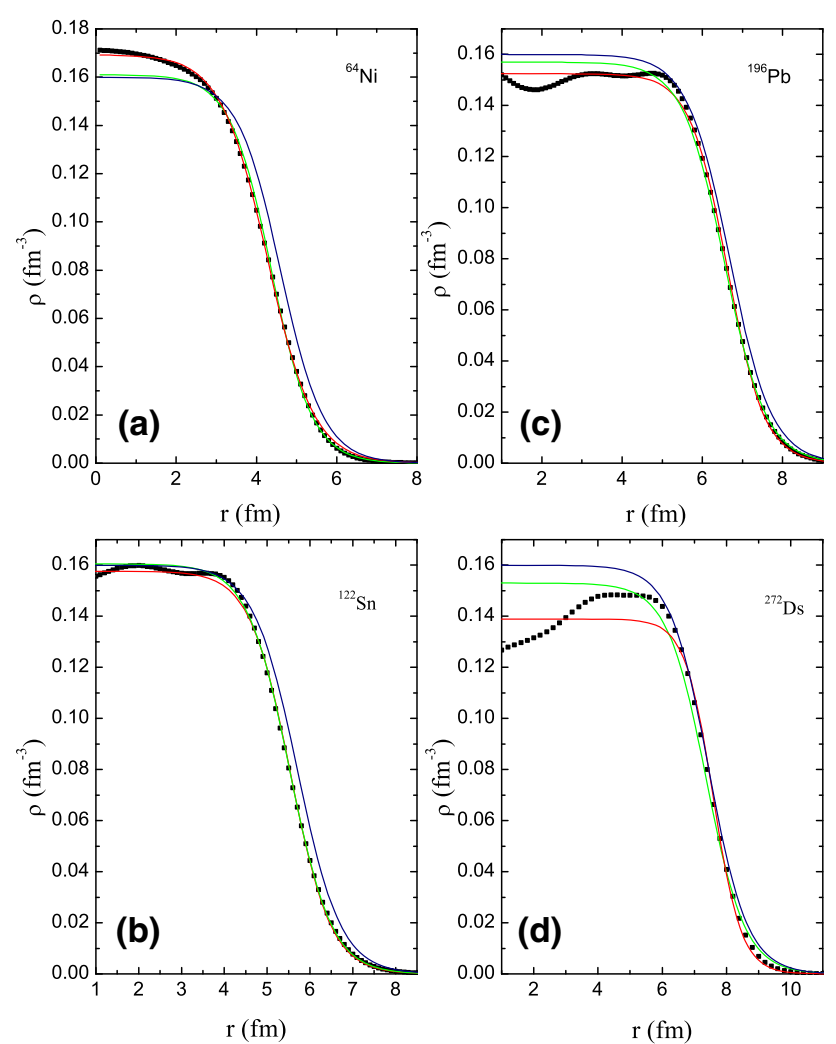

Fig. 38 The nucleon-density distributions calculated with method I in indicated spherical nuclei are fitted with Eq. (99). The results of threeparameter fit are shown by red lines. The results of two-parameter fit at $\rho_{0}$ defined from Eq. (100) are shown by green lines. The results of one-parameter fit at fixed $\rho_{0}=0.16 \mathrm{fm}^{-3}$ and $r_{0}=1.15 \mathrm{fm}$ are shown by blue lines

Wiringa et al. [291]. The results are displayed in Fig. 37 [292]. With that parameter set the masses and radii of spherical nuclei from calcium to lead were well described. Later, in Ref. [147], the spin-orbit and effective tensor components of this EDF were modified to describe nuclei heavier than lead. The corresponding EDF, named DF3-a, turned out to be successful in describing uranium and transuranium nuclei without spoiling the accuracy of description of lighter nuclei. In fact, the Fayans approach allows one to describe successfully the ground-state properties of nuclei from the calcium region to the transuranium one. Nuclear charge radii are reproduced quite accurately on a level of 0.01-0.02 fm [293]. The same is true for nuclear magnetic [294,295] and quadrupole [296$298]$ moments as well as the energies and $B(E 2)$ values for the first $2^{+}$excitations in semi-magic nuclei $[296,299]$. The single-particle energies in doubly magic nuclei are describe with unprecedented accuracy [300]. A comprehensive comparison with several SkEDF together with a discussion of the merits and achievements and possible extensions of the FEDF approach is found in Ref. [301].

\subsection{Nucleon-density distribution}

In the calculation of the nucleus-nucleus potentials we parameterize the self-consistent density distributions found for the spherical nucleus with the Methods I and II by properly normalized three-parameter symmetrized Fermi functions

$\rho(r)=\frac{\rho_{0}}{1+\exp [(r-R) / a]}$,

where $\rho_{0}$ is the saturated nucleon density in the center of nucleus, $R=r_{0} A^{1 / 3}$ is the nuclear radius with the parameter $r_{0}$, and $a$ is the nuclear diffuseness. As seen in Fig. 38, one can fit well the density profile with Eq. (99). The value of

$\rho_{0}=\frac{3}{4 \pi r_{0}^{3}} \frac{1}{1+\left(\frac{\pi a}{R}\right)^{2}}$,

provides the proper normalization of Eq. (99). Using the values of $r_{0}$ and $a$ obtained in three-parameter fit of the nuclear density profile in Fig. 38, we obtain from (100) $\rho_{0}=0.158$, $0.162,0.156$, and $0.154 \mathrm{fm}^{-3}$ for ${ }^{64} \mathrm{Ni},{ }^{122} \mathrm{Sn},{ }^{196} \mathrm{~Pb}$, and ${ }^{276} \mathrm{Ds}$, respectively. These values of $\rho_{0}$ differ from those of the three-parameter fit (Fig. 38). So, the three-parameter fit provides $\rho(r)$, which is not normalized to the total number of nucleons. Using $\rho_{0}$ from Eq. (100), we fit the nucleondensity profile with $r_{0}$ and $a$ and obtain the normalized $\rho(r)$. As seen in Fig. 38, the values of $\rho_{0}$ obtained in this way are close to $0.16 \mathrm{fm}^{-3}$ resulting from the consideration of infinite nuclear matter and the variation of $\rho_{0}$ has minor influence the tail of the density distribution. To reveal the isotopic trends in $r_{0}$ and $a$ and simplify the calculations of nucleusnucleus potential, one can take safely $\rho_{0}=0.16 \mathrm{fm}^{-3}$ up to an accuracy of a few percent and consider the fit of the density profile with $r_{0}$ and $a$. Taking this $\rho_{0}$, we focus on the quality of the description of the nucleon-density distribution at $r>0.8 R$ (Fig. 38). Because the nuclear density tail is mainly responsible for the nucleus-nucleus interaction, the quality of the nuclear-structure input can be checked in the calculation of the nucleus-nucleus potential. Indeed, the height of the Coulomb barrier is a measurable value.

If we fix the values of $\rho_{0}=0.16 \mathrm{fm}^{-3}$ and $r_{0}=1.15 \mathrm{fm}$, the description of the density tail becomes worse (Fig. 38), in particular in the case of light nuclei. In a heavy nucleus, the density profile can be well fitted even at fixed $\rho_{0}$ and $r_{0}$.

For further consideration, we use the two-parameter fit and set $\rho_{0}=0.16 \mathrm{fm}^{-3}$ because this value is close to that obtained in the two-parameter fit resulting in the normalized $\rho(r)$. If the value of $\rho_{0}$ is fixed in (99), the fit of nucleondensity profile results in smoother dependence of $a$ on $N$. Although the values of $a$ are slightly larger in this fit, the qualitative dependence on $N$ remains.

We found that $r_{0}$ is weakly dependent on the neutron number of nucleus. Based on the calculations of nucleon-density 


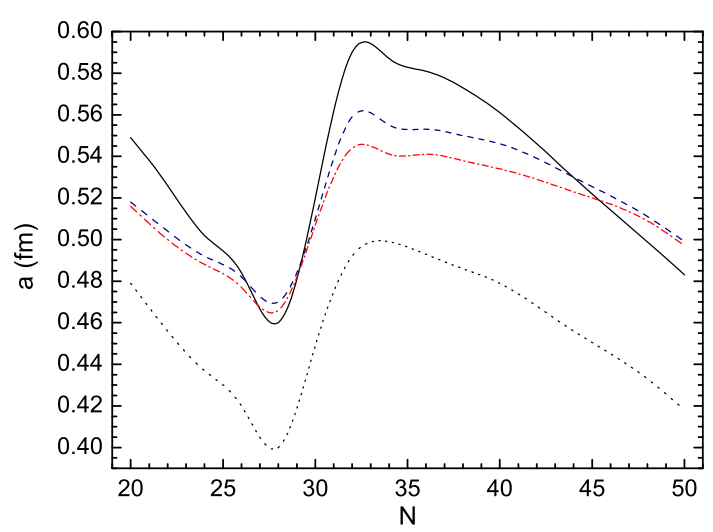

Fig. 39 The comparison of isotopic dependencies of diffuseness of Ni obtained with method I (solid line), method II (dotted line), and approach of Ref. [277] (dashed and dash-dotted lines resulted from the SLy4 and SKMS interactions, respectively)

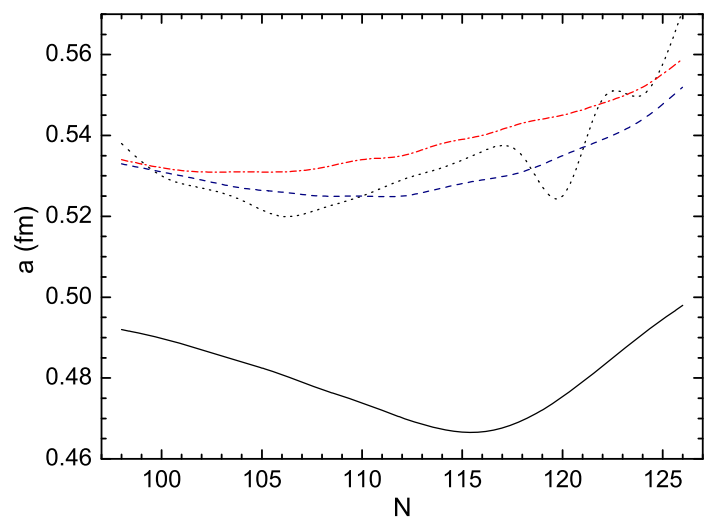

Fig. 40 The same as in Fig. 39, but for $\mathrm{Pb}$

profiles, the following parametrization:

$r_{0}=Z^{1 / 37}(\mathrm{fm})$,

is suggested as a simple, but surprisingly accurate description of the reduced radius parameter. In accordance with (101), the value of $r_{0}$ varies from $1.07 \mathrm{fm}$ for $\mathrm{Mg}$ to $1.135 \mathrm{fm}$ for Ds. Note that this unusual expression provides the values of $r_{0}$ close to those from more sophisticated parametrization given below.

In Figs. 39 and 40, the isotopic dependencies of the diffuseness $a$ in Eq. (99) are shown. The present results and those obtained in Ref. [277] demonstrate the same qualitative dependence on the neutron number. The diffuseness is minimal at $N=28$ and 50 corresponding to the magic numbers. As in Ref. [302], the $a(N)$ dependence in $\mathrm{Pb}$ is essentially nonlinear and is characterized by an increase with $N$ from 112-116 to 126 (Fig. 40). The value of $a$ depends on the strength of $N N$ forces at small nucleon density. This strength is mainly defined by the external $N N$ interaction. The density dependence of $N N$ forces influences the diffuseness as well. As shown, various $N N$ interactions or energy-density functionals provide good description of the nuclear proper- ties but result in quite different values of $a$. In Figs. 39 and 40 , the values of $a$ calculated within different methods vary within factor of 1.2. In Ref. [151], the diffuseness parameter for $\mathrm{Ni}$ is essentially a step function: $a \approx 0.45 \mathrm{fm}$ for $N<40$ and $a \approx 0.5 \mathrm{fm}$ for larger $N$. Although the values of $a$ are close to those obtained in Ref. [151], the qualitative dependence on $N$ is rather different that reflects the dependence of $a$ on the nucleon-nucleon interaction used.

Based on the calculations of proton and neutron densities, the following expressions:

$$
\begin{aligned}
R_{p} & =1.249 A^{1 / 3}-0.5401-0.9582 \frac{N-Z}{A}(\mathrm{fm}), \\
R_{n} & =1.2131 A^{1 / 3}-0.4415+0.8931 \frac{N-Z}{A}(\mathrm{fm}), \\
a_{p} & =0.4899-0.1236 \frac{N-Z}{A}(\mathrm{fm}), \\
a_{n} & =0.4686+0.0741 \frac{N-Z}{A}(\mathrm{fm}),
\end{aligned}
$$

can be suggested to estimate the proton $R_{p}$ and neutron $R_{n}$ radii as well as the diffuseness for protons $a_{p}$ and neutrons $a_{n}$. These expressions are suitable for estimating the smooth part of isotopic dependence of nuclear radius and diffuseness. As seen in Fig. 41, the nuclear radii for protons and neutrons are well reproduced. The proton (neutron) diffuseness is rather well described for the nuclei with $N$ near the magic values. The nuclear radius is estimated as $R=\left(Z R_{p}+N R_{n}\right) / A$, which results in $r_{0}=1.10-1.12 \mathrm{fm}$ for the $\mathrm{Ni}$ isotopes considered. Equation (101) leads to $r_{0}=1.1 \mathrm{fm}$. As seen, the addition dependence of $r_{0}$ on $N$ in (102) causes about $2 \%$ correction to the value of $r_{0}$ obtained with Eq. (101).

As seen in Figs. 42, 43, 44, and 45 the diffuseness calculated with the methods I and II are different. The method I provides larger $a$ for Ni. However, it results in smaller values of $a$ for $\mathrm{Pb}$ and Ds than those from the method II. For Ni and Sn, the functions $a(N)$ have the minima at $N=28,50$, and 82. There is also the minimum of $a$ at $N=162-164$ in Ds. In the single-particle schemes of spherical nuclei, these neutron shells and sub-shells are closed by the levels with large orbital angular momenta $(l \geq 7)$ [39]. Thus, the centrifugal barrier restricts the diffuseness of some closed shell nuclei. For $\mathrm{Pb}$, the magic neutron number $N=126$ is not reflected in the $a(N)$ dependence because this shell is closed by the level with small orbital angular momenta. So, one can conclude that the last occupied single-particle level is responsible for the peculiarities in isotopic dependence of the nuclear diffuseness. The same was pointed out in Ref. [276].

The dependencies of $a$ on $N$ in Figs. 42, 43, 44, and 45 are rather complicated to be fitted with a simple formula. As in Refs. [277,278,303], one can consider the dependence of $a$ on the neutron separation energy $S_{n}$. Indeed, the value of $a$ is proportional to $1 / \sqrt{S_{n}}$. In this case one can suggest the 


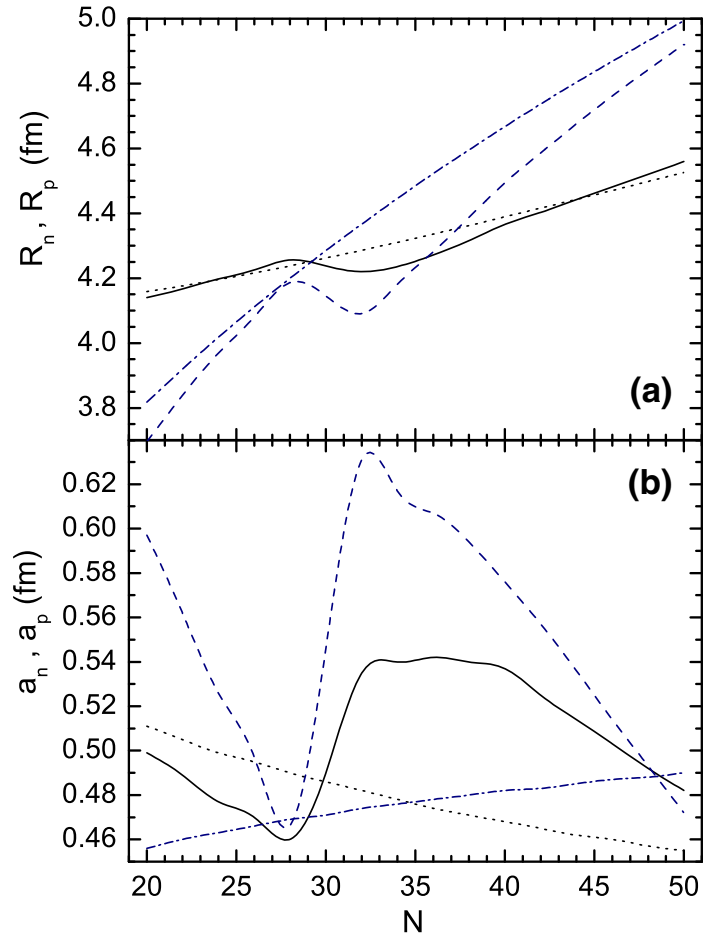

Fig. 41 Comparison of isotopic dependencies of proton (solid line) and neutron (dashed line) radii (a), and proton (solid line) and neutron (dashed line) diffuseness (b) obtained with method I for Ni isotopes with those calculated for protons (dotted lines) and neutrons (dashdotted lines) with Eqs. (102)

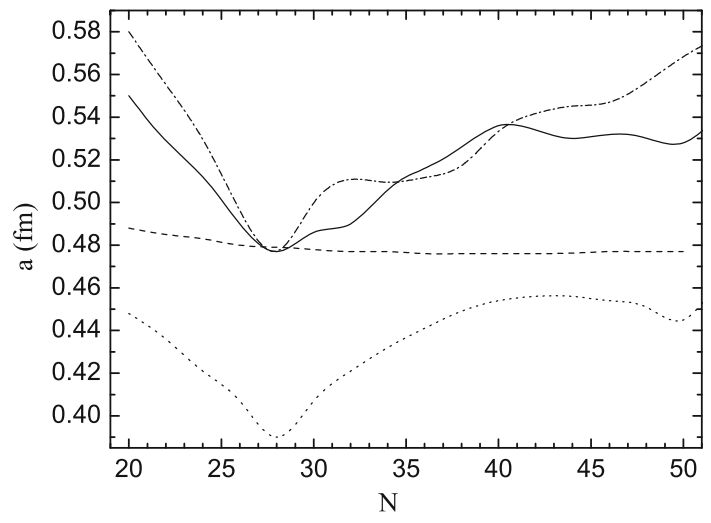

Fig. 42 The comparison of isotopic dependencies of diffuseness of spherical Ni obtained with method I (solid line) and method II (dotted line). The values of $a$ are obtained at $\rho_{0}=0.16 \mathrm{fm}^{-3}$. The fit of the solid line with Eq. (103) at $N_{0}=28$ is presented by a dash-dotted line. The isotopic dependence of $a$ calculated with Eqs. (102) is presented by a dashed line

simple parametrization

$a(N)=a_{0} \frac{N}{2 N-N_{0}} \sqrt{S_{0} / S_{n}}(\mathrm{fm})$,

where $a_{0}$ and $S_{0}$ are the diffuseness and neutron separation energy, respectively, in the isotope with neutron number $N_{0}$. As seen in Figs. 42 and 43, this expression allows us to esti-

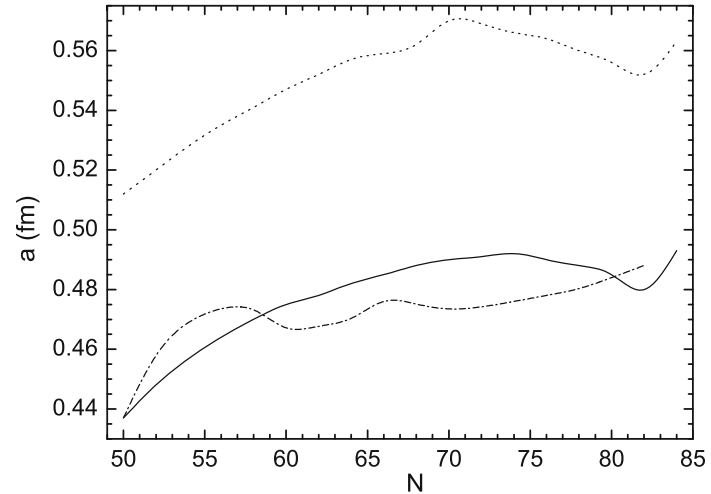

Fig. 43 The same as in Fig. 42, but for spherical Sn. The fit of the solid line with Eq. (103) at $N_{0}=50$ is presented by a dash-dotted line

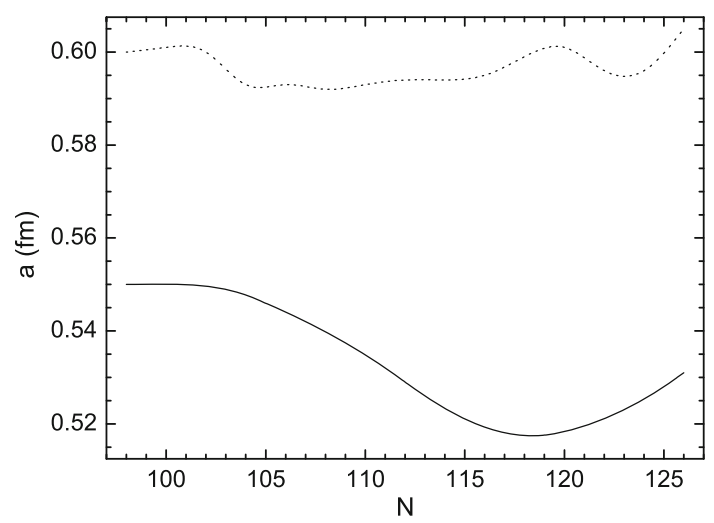

Fig. 44 The same as in Fig. 42, but for spherical $\mathrm{Pb}$

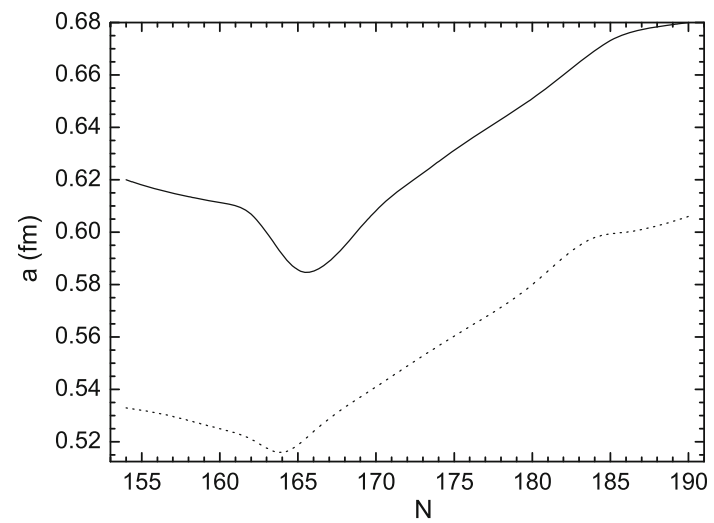

Fig. 45 The same as in Fig. 42, but for spherical Ds

mate a diffuseness with satisfactory accuracy. Although the value of $N_{0}$ can be arbitrary chosen, we set $N_{0}=Z$ in Figs. 42 and 43. Equation (103) provides a better evaluation of $a$ for $N$ closer to $N_{0}$ and heavier nuclei. In Fig. 42, the isotopic dependence of $a=\left(Z a_{p}+N a_{n}\right) / A$ is presented, where the values of $a_{p}$ and $a_{n}$ are calculated with Eq. (102). For most of the isotopes, the accuracy of this parametrization is within $15 \%$. 


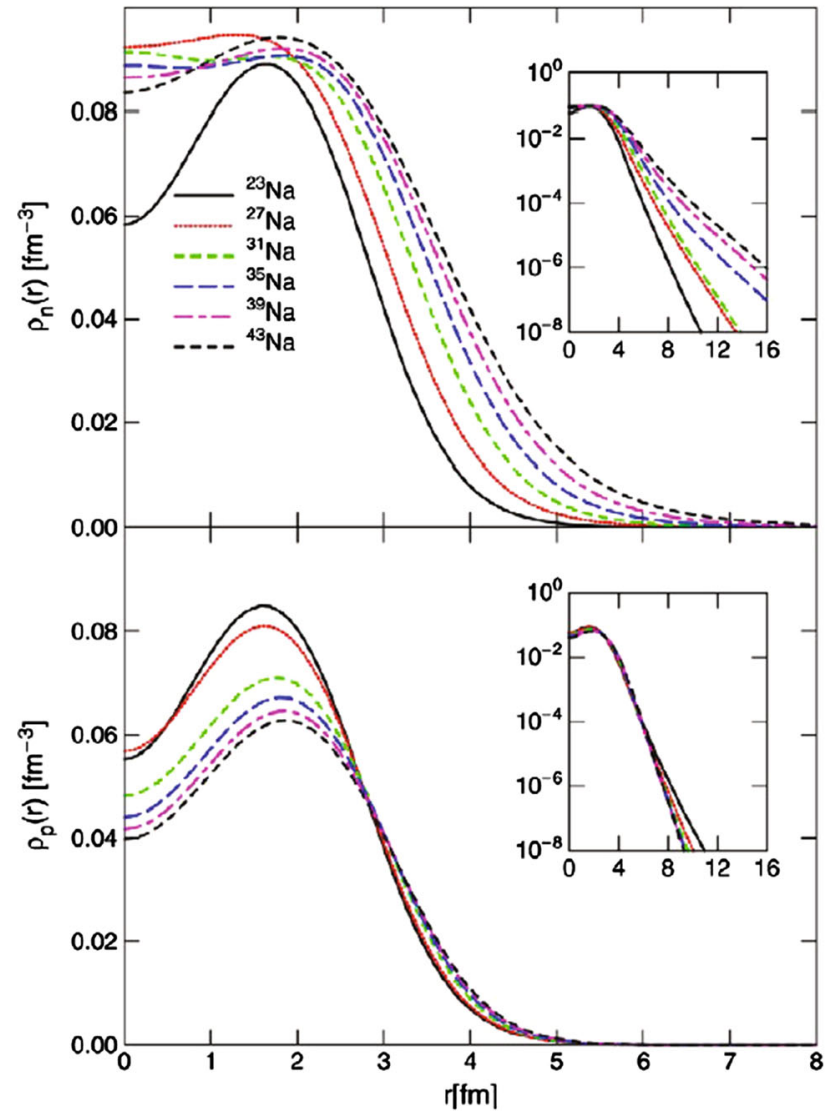

Fig. 46 The calculated neutron (upper) and proton (lower) density distributions in $\mathrm{Na}$ isotopes [304]. The same figures but in logarithm scale are given as inserts, to show the tail part of the density distribution

The density distributions have been examined within the $\mathrm{RCHB}$ theory for $\mathrm{Na}$ isotopes in Ref. [304]. The calculated density distributions for both protons and neutrons in $\mathrm{Na}$ isotopes are shown in Fig. 46. As seen in the upper part, with increasing neutron number $N$, the density near the center and the neutron radius increase due to the occupation of the $2 s_{1 / 2}$ level. In the proton density, the surface is more or less unchanged because of the Coulomb barrier, but with increasing $N$ the density of the center decreases due to the slightly increasing tail. This occurs due to the symmetry energy. But as the density must be multiplied by a factor $4 \pi r^{2}$ before the integration in order to give a fixed $Z$, the change in the center slightly influences the outer part of the proton distribution.

It should be stressed that the asymptotic tails of the density distributions mainly contribute to the folding integral contained in the expression for the nuclear part of the nucleusnucleus potential. Therefore, the differences between the self-consistent density distributions and the Fermi ones, which can be in the central regions, are expected to be unimportant for the results.

\subsection{Nucleus-nucleus interaction potential}

The Fayans EDF belongs to the class of finite-range functionals with Yukawa-type coordinate dependence of the central force $[146,284,293,296]$. The in-volume central term of the EDF can be schematically written as

$\mathcal{E}_{\mathrm{v}}^{(\mathrm{Fy})}\left(x_{0}\right)=\frac{C_{0} F_{e x} x_{0}^{2}}{2} \frac{1+\alpha x_{0}^{\sigma}}{1+\gamma x_{0}}$.

In order to account properly for the particle number in Eq. (104), we use the scaled strength $C_{0}=C_{0}^{\prime} \rho_{10} \rho_{20} / \rho_{0}^{2}$, where $\rho_{10}$ and $\rho_{20}$ are defined by (100) for interacting nuclei. The constant $C_{0}^{\prime}=339 \mathrm{MeV} / \mathrm{fm}^{3}$ is related to $\rho_{0}=0.16$ $\mathrm{fm}^{-3}$ [171], the parameters $F_{e x}, \alpha, \sigma$, and $\gamma$ are chosen such that nuclear properties are described. The $N N$ interaction corresponding to Eq. (104) is

$$
\begin{aligned}
F= & \frac{\delta^{2} \mathcal{E}_{\mathrm{v}}^{(\mathrm{Fy})}\left(x_{0}\right)}{\delta x_{0}^{2}}=C_{0} \frac{F_{e x}}{2\left(1+\gamma x_{0}\right)^{3}}\left[2+2 \alpha x_{0}^{\sigma}\right. \\
& \left.+\alpha \sigma x_{0}^{\sigma}\left(1+\gamma x_{0}\right)\left(3+\sigma+\gamma x_{0}(1+\sigma)\right)\right] .
\end{aligned}
$$

This expression is obtained by taking the second derivative of (104) with respect to $x_{0}$. At $x_{0} \rightarrow 0$ we get an external $N N$ interaction and $F \rightarrow C_{0} F_{e x}$. The $N N$ interaction at saturation density is found for $x_{0} \rightarrow 1$.

Varying the parameters in (105), one can obtain different forms of the $N N$ interaction. For example, at $\gamma=0$ and $\sigma=1$,

$F=C_{0}\left[F_{e x}+\left(F_{i n}-F_{e x}\right) x_{0}\right]$,

has the Migdal form [171] with $F_{i n}=F_{e x}(1+3 \alpha)$.

The nucleus-nucleus interaction potential $V$ is represented as the sum [305]

$V(R)=V_{\mathrm{C}}(R)+V_{N}(R)+V_{R}(R)$

of the Coulomb $V_{\mathrm{C}}$, nuclear $V_{N}$, and centrifugal potentials. While $V_{\mathrm{C}}$ and $V_{R}$ have analytical forms, the calculation of the potential $V_{N}(R)$ is the one most difficult. We calculate it in accordance with the double-folding procedure as

$V_{N}(R)=\int d \mathbf{r}_{1} d \mathbf{r}_{2} \rho_{1}\left(\mathbf{r}_{1}\right) \rho_{2}\left(\mathbf{R}-\mathbf{r}_{2}\right) F\left(\mathbf{r}_{2}-\mathbf{r}_{1}\right)$.

The effective $N N$ forces, Eq. (106),

$F\left(\mathbf{r}_{2}-\mathbf{r}_{1}\right)=C_{0}\left(F_{i n} x\left(\mathbf{r}_{1}\right)+F_{e x}\left[1-x\left(\mathbf{r}_{1}\right)\right]\right) \delta\left(\mathbf{r}_{\mathbf{2}}-\mathbf{r}_{1}\right)$,

depend on the total nuclear density $\rho\left(\mathbf{r}_{\mathbf{1}}\right)=\rho_{1}\left(\mathbf{r}_{\mathbf{1}}\right)+\rho_{2}(\mathbf{R}-$ $\left.\mathbf{r}_{2}\right)$. The interaction strengths are defined by the LM parameters $F_{i n}=0.09$ and $F_{e x}=-2.59 ; C_{0}=300 \mathrm{MeV} \mathrm{fm}^{3}$ $\left(\rho_{0}=0.17 \mathrm{fm}^{3}\right)$ were determined from a fit to experimentally measured properties of nuclei [171]. The values employed in our previous calculations with (109) fell within the range $r_{0}=1.15-1.16 \mathrm{fm}$ for the nuclear radii and within the range 

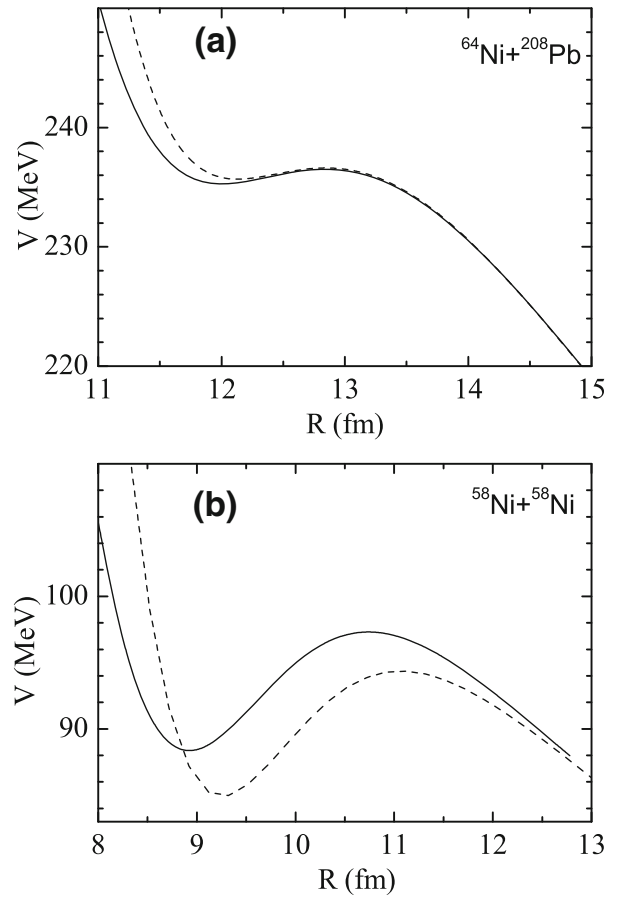

Fig. 47 Comparison of the nucleus-nucleus potentials calculated with Eq. (105) and using nucleon densities of method I (solid lines), and with Eq. (109) and adopted $\rho$ (dashed lines) for the reactions indicated. The parameters are given in the text

$a=0.53-0.56 \mathrm{fm}$ for the diffuseness parameters, depending on the nuclear mass. For the alpha-particle, $r_{0}=1.01 \mathrm{fm}$ and $a=0.47 \mathrm{fm}$ were used. These values allow us to describe rather well the height $V_{B}$ of the Coulomb barriers in various reactions. However, in several reactions the Coulomb barriers are underestimated in our calculations. For example, in the ${ }^{58} \mathrm{Ni}+{ }^{58} \mathrm{Ni}$ reaction the calculated $V_{B}$ was about $3 \mathrm{MeV}$ smaller than the experimental value. As shown, for example in Ref. [305], the tail of the total nucleon density $\rho$ mainly influences the value of $V_{N}$. The role of $\rho_{1}$ is less important because its contribution to $V_{N}$ is proportional to $(N-Z) / A$.

As seen, the values of $a$ and $r_{0}$ found in the present work are smaller than those used in our previous calculations of the nucleus-nucleus potential with (109). If they were used with the interaction (109), the Coulomb barriers would be higher than those resulting from the experimental data. Using the values of $a$ and $r_{0}$ found, we calculate the nucleus-nucleus potential with $F$ defined in the general form (105). As in Ref. [146], we set $\sigma=1 / 3$ and look for the values of $F_{e x}, \alpha$, and $\gamma$ which provide a good description of the Coulomb barrier heights.

It was found that with $F_{e x}=-10.8, \alpha=-0.534$, $\gamma=0.4$, and nucleon densities obtained with method I the calculated nucleus-nucleus potentials provide correct values of the Coulomb barrier height and are close to those obtained with the Migdal interaction Eq. (109). In the ${ }^{64} \mathrm{Ni}+{ }^{208} \mathrm{~Pb}$ reac-
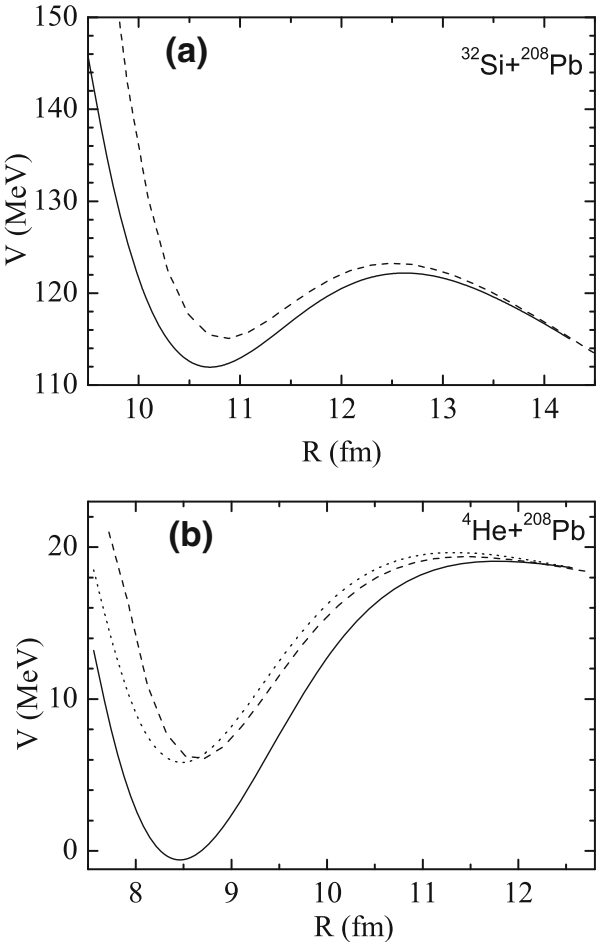

Fig. 48 The same as in Fig. 47, but for the asymmetric reactions indicated. The dotted line is obtained with the same parameters as the solid line, but with $a=0.39$ fm for ${ }^{4} \mathrm{He}$

tion the present potential and that calculated previously with Eq. (109) almost coincide (Fig. 47). In the ${ }^{58} \mathrm{Ni}+{ }^{58} \mathrm{Ni}$ reaction (Fig. 47), the shapes of the potentials are almost the same but the present calculation results in the $3 \mathrm{MeV}$ higher Coulomb barrier that is closer to that required to describe the fusion excitation function at energies near the barrier [306]. Note that the height of the Coulomb barrier is precisely defined by measuring the fusion excitation function.

With the parameters used we obtain $F_{\text {in }}=0.062$, which is relatively close to the internal constant of the interaction Eq. (109). The strength $\left|F_{e x}\right|$ of the external nucleon-nucleon interaction is larger than in the Migdal interaction. Indeed, the calculated nucleon-density distribution has smaller diffuseness because of the stronger nucleon-nucleon attraction used.

For the ${ }^{32} \mathrm{~S}+{ }^{208} \mathrm{~Pb}$ and ${ }^{4} \mathrm{He}+{ }^{208} \mathrm{~Pb}$ systems, the nucleusnucleus potentials obtained with nucleon densities discussed in this paper are quite similar to those calculated with the prescription of Ref. [305] for asymmetric nuclear systems (Fig. 48). The calculations with self-consistent nucleon densities result in a $2.3-6.5 \mathrm{MeV}$ deeper potential pocket in the nucleus-nucleus potential and in a slightly smaller height, $0.3-1 \mathrm{MeV}$, of the Coulomb barrier. Because we did not calculate the diffuseness for ${ }^{4} \mathrm{He}$, the sensitivity of the results to its variation is demonstrated in Fig. 48. 


\section{Polarization of nuclear surface in deformed nuclei}

There are extensive discussions currently on the difference of the proton-neutron nuclear radius to study the symmetry energy [185,270-272]. Such considerations are usually restricted to spherical nuclei. Most nuclei, however, are found to be deformed in their ground states, especially in the medium- and heavy-mass region [307,308]. So, the surface properties of these nuclei can depend on the deformation and, if rotational symmetry is broken, even on the azimuthal angle.

Particular attention is taken to study the diffuseness of the surface which have an important influence for the calculation of deformation $[309,310]$ as well as for the calculation of the nucleus-nucleus interaction and fission barrier [311]. While the central density and radius can be understood with rather simple arguments, properties of the nuclear surface diffuseness turns out to be more complicated than anticipated.

The nuclear density obtained with the EV8 code [312] was investigated over 749 even-even nuclei ranging from $Z=8$ to $Z=108$ whose experimental masses are known. The $E V 8$ code solves the HF+BCS in $r$-space with the Skyrme functional in the mean-field term and with a contact interaction in the pairing channel. Here a surface type pairing interaction is used with the same strength as in Ref. [313]. Two different functionals, namely SLy4 [314] and the SkM* [315] have been employed in the mean-field channel. The specific symmetries imposed in the EV8 program only allow for even multipole deformation: quadrupole, hexadecapole, etc. The number of nuclei that are found to be spherical, oblate, prolate, or triaxial with the $\mathrm{SkM}^{*}$ functional are, respectively, 346, 59, 290 and 54. The tolerance for the attribution of a given shape is 0.02 and $1^{\circ}$ for $\beta_{2}$ and $\gamma$, respectively.

The multipole parameters, denoted by $\beta_{\lambda}$, are rather global quantities that do not allow for a precise analysis of the local density properties, especially those related to the nuclear surface. To get deeper insight, we have systematically fitted the ground-state densities using a shape given by

$\rho(\mathbf{r})=\frac{\rho_{0}}{1+\exp \left[\frac{|\mathbf{r}|-R(\theta, \varphi)}{a(\theta, \varphi)}\right]}$.

Here $(\mathbf{r}=|\mathbf{r}|, \theta, \varphi)$ are the standard spherical coordinates.

The radius $R(\theta, \varphi)$ is assumed to take the form

$R(\theta, \varphi)=R_{0}\left\{1+\beta_{2} Y_{20}(\theta, \varphi)+\beta_{4} Y_{40}(\theta, \varphi)\right\}$,

where we consider only axially deformed nuclei. As a consequence, the 42 nuclei that are found triaxial are not included in the following analysis. In Eq. (111) the volume conservation is taken in $R_{0}$ into account.

The parametrization that should be taken for the diffuseness parameter is less clear. In particular, there is a subtle aspect related to the fact that $a(\theta, \varphi)$ corresponds to the dif-

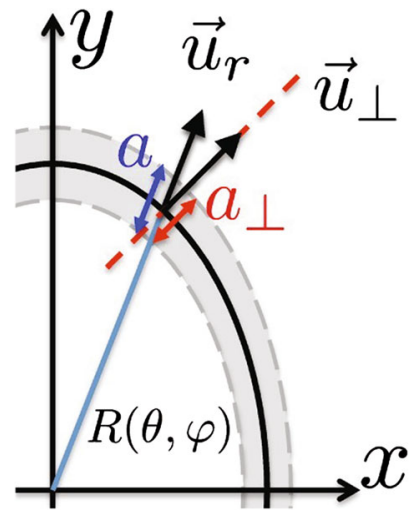

Fig. 49 Two-dimensional schematic illustration of the difference between the diffuseness along the radial axis (along the direction $\mathbf{u}_{r}$ ) and along the axis perpendicular to the isodensity $\rho=\rho_{0} / 2$ (along the direction $\mathbf{u}_{\perp}$ )

fuseness along the radial axis, which is different from the diffuseness perpendicular to the $\rho(|\mathbf{r}|=R(\theta, \varphi))=\rho_{0} / 2$ isodensity surface. The latter diffuseness is denoted by $a_{\perp}(\theta, \varphi)$ below. To illustrate this point, a schematic two-dimensional picture is given in Fig. 49.

In spherical systems, the two diffuseness are identical. However, due to the deformation, even if $a_{\perp}$ is constant, the radial diffuseness becomes angular dependent. This has been briefly discussed in Sect. 4 of Ref. [38]. The general form of the diffuseness is considered as

$a(\theta, \varphi)=a_{\perp}(\theta, \varphi) \sqrt{1+\left.|\nabla R(\theta, \varphi)|_{r=R(\theta, \varphi)}\right|^{2}}$,

that can be easily obtained noting that

$\mathbf{u}_{r} \cdot \mathbf{u}_{\perp}=\frac{1}{\sqrt{1+\left.|\nabla R(\theta, \varphi)|_{r=R(\theta, \varphi)}\right|^{2}}}$,

where the two normalized vectors $\mathbf{u}_{r}$ and $\mathbf{u}_{\perp}$ are displayed in Fig. 49.

Effects beyond the pure geometric ones are included in the angular dependence of $a_{\perp}(\theta, \varphi)$. Here, we assume that this diffuseness can be expressed similarly to Eq. (111) with

$a_{\perp}(\theta, \varphi)=a_{0}\left(1+\tilde{\beta}_{2} Y_{20}(\theta)+\tilde{\beta}_{4} Y_{40}(\theta)\right)$.

Note that a similar parametrization has been proposed in Ref. [316].

\subsection{Parameters of nucleon-density distribution}

For each nucleus, the total local density has been fitted using the parameters $\rho_{0}, R_{0}, a_{0}, \beta_{2}, \beta_{4}, \tilde{\beta}_{2}$, and $\tilde{\beta}_{4}$ in Eq. (110) with the parametrization (112) together with Eqs. (111) and (113). The set of parameters obtained for all considered nuclei are provided in Ref. [277]. In the following, the terminology "polarization of the nuclear surface" will be employed for 


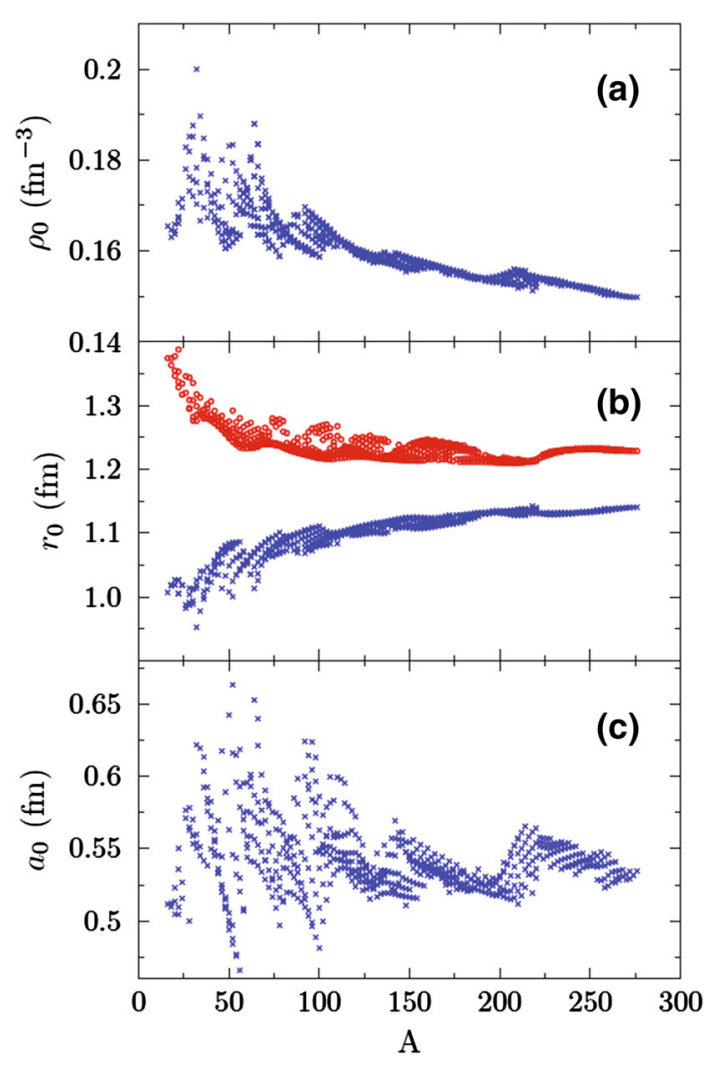

Fig. 50 Values of the density $\rho_{0}(\mathbf{a})$, reduced radius $r_{0}(\mathbf{b})$ and average diffuseness $a_{0}(\mathbf{c})$. In panel $\mathbf{b}$, the equivalent sharp radius defined as $r_{0}=\sqrt{5 / 3\left\langle r^{2}\right\rangle / A^{1 / 3}}$ is also shown (red open circles) for comparison

systems with nonzero values of the $\tilde{\beta}_{2}$ and/or $\tilde{\beta}_{4}$ coefficients. Here, only results using the $\mathrm{SkM}^{*}$ functional [315] are shown. Note that the same analysis performed with the SLy4 [314] functional results in similar conclusions.

The $\rho_{0}, R_{0}, a_{0}$ parameters obtained for the $\mathrm{SkM}^{*}$ are, respectively, shown in Fig. 50 for masses $A$ ranging from 16 to 276. The corresponding deformations parameters are presented in Ref. [277]. Only nuclei that are found to be deformed in EV8 are shown. The central density and extension of nuclei [317-320] have been extensively discussed in the literature, for instance, to study the neutron skin thickness [321,322].

The large value of the central density for low masses is due to the shell structure effect: the filling of the $s_{1 / 2}$ level increases the central density in nuclei. For large masses, this effect is less important; however, the average density tends to decrease due to the asymmetry term proportional to $\delta=$ $\frac{N-Z}{A}$, which becomes more effective as the mass increases along the beta stability line [323].

\subsection{Diffuseness polarization}

In Fig. 50c, large finite size effects are uncovered in the fluctuations of $a_{0}$. This is clearly seen in the fluctuations observed
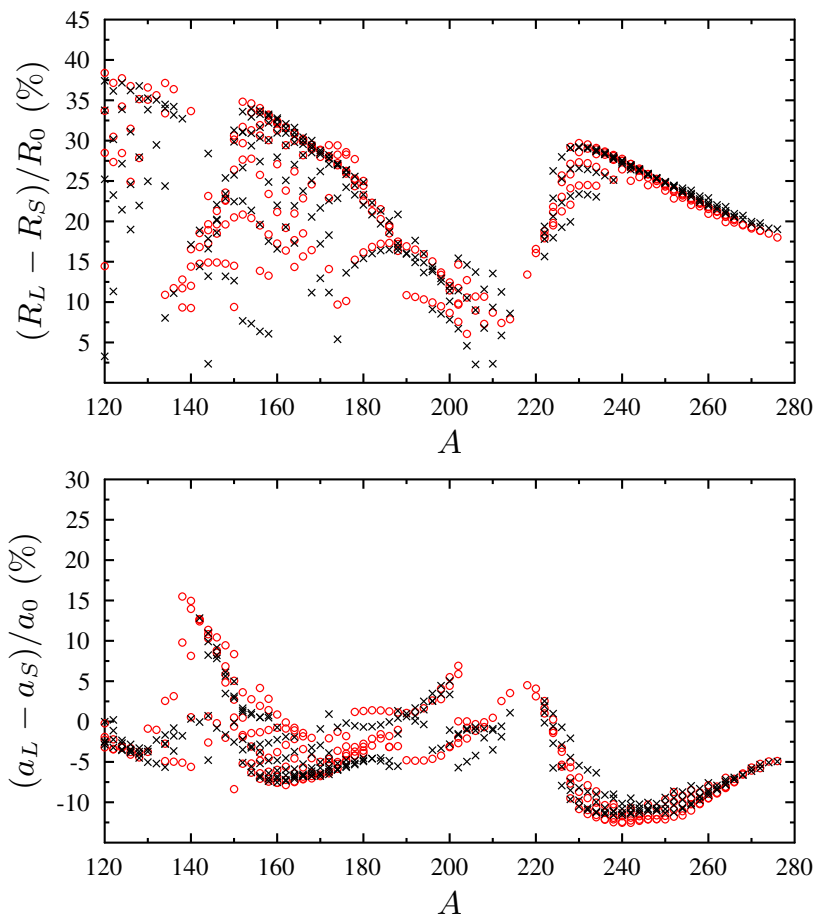

Fig. 51 Top: $\left(a_{L}-a_{S}\right) / a_{0}$ as a function of $\left(R_{L}-R_{S}\right) / R_{0}$ for mass $A>120$. Bottom: $\left(a_{L}-a_{S}\right) / a_{0}$ as a function of mass in $\%$. The black cross and red open circles correspond respectively to results obtained with the $\mathrm{SkM}^{*}$ and SLy4 functional

for masses $A<150$. The finite size effects are stronger than in $\rho_{0}$ and $R_{0}$, with non-vanishing fluctuations around the mean value $\bar{a}_{0} \simeq 0.55 \mathrm{fm}$ even for larger mass. In addition, the structures are clearly seen that stem from the appearance of magic numbers. When the system is deformed, non-trivial distortion of the nuclear surface occurs. This distortion is associated with nonzero values of the $\tilde{\beta}_{2}$ and $\tilde{\beta}_{4}$ parameters, which is called hereafter polarization of the nuclear surface. Comparing with the results of the previous section, one can clearly see the dependence of the diffuseness on the EDF used. However, on average the qualitative results are almost the same.

In the heavy-mass region, we clearly see that an increase of $\beta_{2}$ (with $\beta_{2}>0$ ) leads to an increase of $\left|\tilde{\beta}_{2}\right|\left(\tilde{\beta}_{2}<0\right.$ ). Denoting, by $R_{L}\left(a_{L}\right)$ and $R_{S}\left(a_{S}\right)$ the radius (diffuseness) along the elongated and compressed axis, the two quantities $\left(R_{L}-R_{S}\right)$ and $\left(a_{L}-a_{S}\right)$ are strongly anti-correlated (see Fig. 51) in this mass region. Note that, with the present deformed Fermi densities, we have

$\frac{\left(R_{L}-R_{S}\right)}{R_{0}}=\frac{3}{4} \sqrt{\frac{5}{\pi}} \beta_{2}+\frac{15}{16 \sqrt{\pi}} \beta_{4}$.

with a similar expression for $\left(a_{L}-a_{S}\right) / a_{0}$ where the $\beta$ 's are replaced by the $\tilde{\beta}$ 's. In Fig. 51 , the results obtained using the SLy4 functional are also shown, demonstrating that changing functional leads qualitatively to the same effect. 


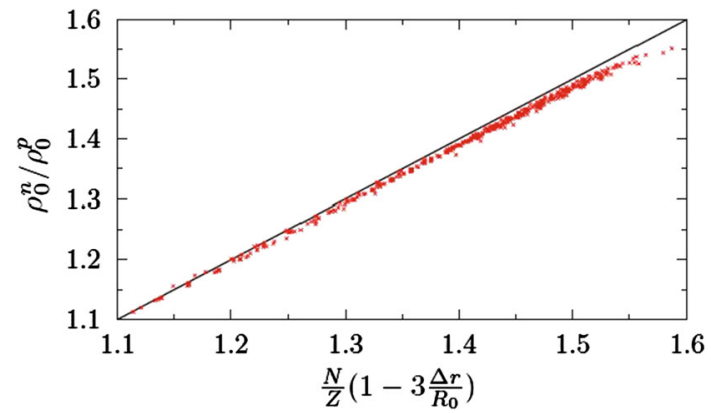

Fig. $52 \rho_{0 n} / \rho_{0 p}$ as a function of $\frac{N}{Z}\left(1-3 \frac{\Delta r}{R_{0}}\right)$. The $x=y$ line is added as a guidance. Only nuclei with $A>120$ are shown

Therefore, in heavy systems, one can systematically observe the following phenomenon: as the system becomes more and more deformed, its diffuseness along the elongated axis becomes smaller in favor of an increase of the diffuseness along the compressed axis. Such a distortion of the nuclear surface is a highly non-trial effect that stems from a complex mixing of the volume, surface and Coulomb field entering in the EDF in the presence of pairing. Surprisingly enough, this effect does not seem to be negligible and can lead to an overall fluctuations of $10 \%$ of the diffuseness along the isodensity surface contour (Fig. 51 (bottom)).

Possible $N / Z$ dependence of the nuclear density profiles can be analyzed by considering separately the neutron and proton densities, denoted by $\rho_{\tau}(\mathbf{r})$, with $\tau=n, p$, respectively for neutron and proton. The set of parameters obtained for all considered nuclei separately for neutrons and protons are provided in the supplemental material of Ref. [277].

As known, when the $N / Z$ ratio changes, the neutron skin thickness can be related to the symmetry energy [270-272]. We give here specific aspects related to the use of EDF in combination with the Fermi shape analysis of the density profile. Let us assume in first approximation that nuclei can be considered as non-deformed nuclei and that the two proton and neutron Fermi liquids present both an equivalent sharp radius, denoted by $R_{n}$ and $R_{p}$. The average densities will verify $\rho_{0}^{n} / \rho_{0}^{p}=N / Z\left(R_{n} / R_{p}\right)^{3}$, leading to

$\frac{\rho_{0}^{n}}{\rho_{0}^{p}} \simeq \frac{N}{Z}\left(1-3 \frac{\Delta r}{R_{0}}\right)$,

where $R_{0}$ is the equivalent sharp radius of the nuclear drop while $\Delta r=R_{n}-R_{p}$ is the neutron skin thickness. Figure 52 illustrates that this relation is perfectly fulfilled and that the contributions of the surface and deformation on average densities are almost negligible.

There is evident correlation between $\beta_{2 n}\left(\beta_{4 n}\right)$ and the $\beta_{2 p}$ $\left(\beta_{4 p}\right)$ [277]. One can draw the following conclusions: (1) The shape deformation of nuclei appear to be almost independent on the fact that protons, neutrons or both are considered, i.e. $\beta_{x n} \simeq \beta_{x p} \simeq \beta_{x}$ with $x=2$ or 4 . Note that this conclusion does not hold for particular deformed halo nuclei in which the shape decoupling effects have been predicted [324-328]. (2) The diffuseness properties depends explicitly on the neutron/proton nature of the considered fluid. Not surprisingly due to the absence of the Coulomb field, neutrons densities are generally more diffuse than those of protons. (3) Denoting by $X_{\tau}$ one of the parameters $\left(a_{\tau}, \beta_{2 \tau}\right.$ or $\left.\beta_{4 \tau}\right)$ associated to the diffuseness and $X$ the equivalent quantity obtained by fitting the total density, to a good approximation, we found that

$X \simeq\left(\frac{N}{A}\right) X_{n}+\left(\frac{Z}{A}\right) X_{p}$.

\subsection{Role of surface polarization effects in nuclear dynamics}

The onset of deformation induces systematically a polarization of the nuclear surface diffuseness that depends on the isospin. Such a polarization has sometimes been suspected to affect nuclear spectroscopy. For instance, in Ref. [329], the surface diffuseness polarization has been proposed as a source of reduction of the dipole strength in the low-lying energy sector. Another anticipated effects is a possible modification of the fusion barrier due to the change of the diffuseness. This aspect might be particularly crucial for reactions involving very heavy systems generally used in the synthesis of the superheavy nuclei.

Consistently with the double-folding approach and/or proximity potential approach, a change in the nuclear diffuseness is anticipated to influence the Coulomb barrier properties. Here, we give an illustration for the reaction ${ }^{40} \mathrm{Ca}+{ }^{238} \mathrm{U}$ (Fig. 53). The uranium nuclei have been shown to present significant deformation in their ground states inducing diffuseness polarization. To estimate the impact of the diffuseness change on nuclear dynamics, the nucleus-nucleus interaction potential $V(R)$ has been estimated using three different values of the diffuseness parameter using the procedure presented in Refs. [205,306,330]. For the nuclear part of the nucleus-nucleus potential, the double-folding formalism with density-dependent effective nucleon-nucleon interaction is used. Within this formalism many heavy-ion capture reactions with stable and radioactive beams at energies above and well below the Coulomb barrier have been successfully described [331]. Here, the quadrupole and hexadecapole deformations of the ${ }^{238} \mathrm{U}$ are taken into account. For spherical nucleus, a diffuseness $a=0.56 \mathrm{fm}$ is used while for the deformed ${ }^{238} \mathrm{U}$, the three values $a=0.494,0.5504$, and $0.5832 \mathrm{fm}$ correspond, respectively, to the minimal, average and maximal values of the radial diffuseness. As seen from this figure, the change of the diffuseness can induce an increase or a decrease of the fusion barrier and will ultimately modify the capture cross-section. An increase of the barrier is anticipated for zero relative angle due to the lower diffuse- 


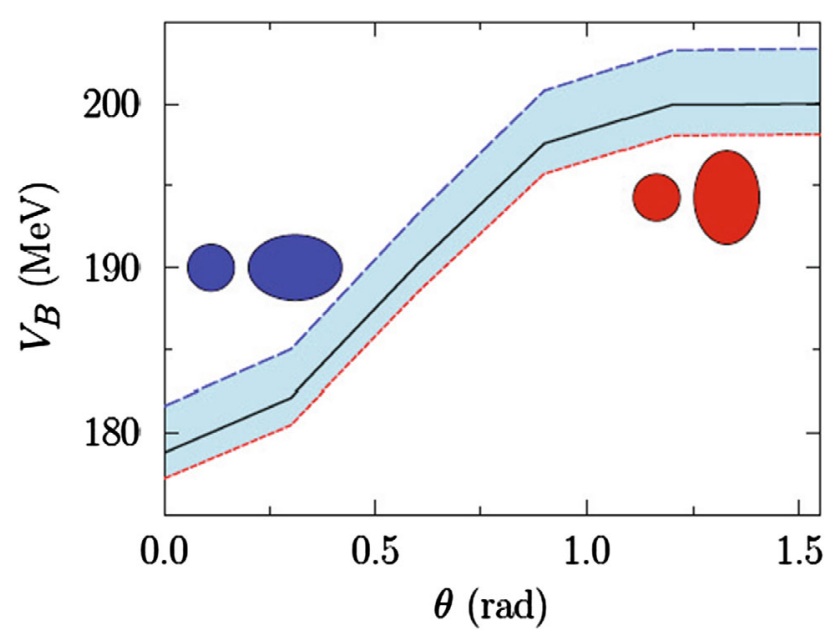

Fig. 53 The calculated dependencies of the Coulomb barrier heights $V_{B}$ on the mutual orientation of colliding nuclei ${ }^{40} \mathrm{Ca}$ and ${ }^{238} \mathrm{U}$. The three curves have been obtained using a diffuseness parameter of $a=$ 0.494 (blue long dashed line), 0.5504 (black solid line) and 0.5832 $\mathrm{fm}$ (red short dashed line) and deformation parameter for the ${ }^{238} \mathrm{U}$ are $\beta_{2}=0.244, \beta_{4}=0.094$. The filled area is shown to underlines the possible change in the fusion barrier

ness along the main axis of deformation, while at $\theta=\pi / 2$ the barrier will be reduced (Fig. 53). The influence of this effect on the nucleus-nucleus interaction deserves further investigations. Note that the saturation at large $\theta$ is due to the interplay between the nuclear and Coulomb interactions.

\subsection{Correlation between observed $\alpha$-decays and changes in} neutron or proton skins from parent to daughter nuclei

The experimental work on the proton and neutron density distributions of stable and exotic nuclei is mainly focusing on the measurement of their rms radii and neutron skin thicknesses. The correlations are found between the neutron skin thicknesses and the electric dipole polarizability, the isoscalar giant quadrupole and pygmy resonances, the neutron removal cross-section, the nuclear surface polarization, "scissors" vibrational modes and the slope of the symmetry energy and consequently with the pressure of neutron matter at saturation density [125]. In Ref. [332], the change of proton and neutron thickness is studied in nuclei after $\alpha$-decay. The skin thicknesses are self-consistently calculated within the SkHFB approach. The decays of the proton-skinned $\alpha$ emitters preferably proceed to yield significant decrease in the proton skins of their daughter nuclei. The reduction in the proton skin thicknesses due to the $\alpha$-emission from parent isotopes increases with neutron number $N$ (with decreasing their proton skins).

Figure 54a shows the neutron skin difference $\delta_{n \alpha}(A, Z)$ versus the corresponding neutron number for the ${ }^{140-155} \mathrm{Gd}$ isotopes [332]. The heavier isotopes of $\mathrm{Gd}$ possess negative

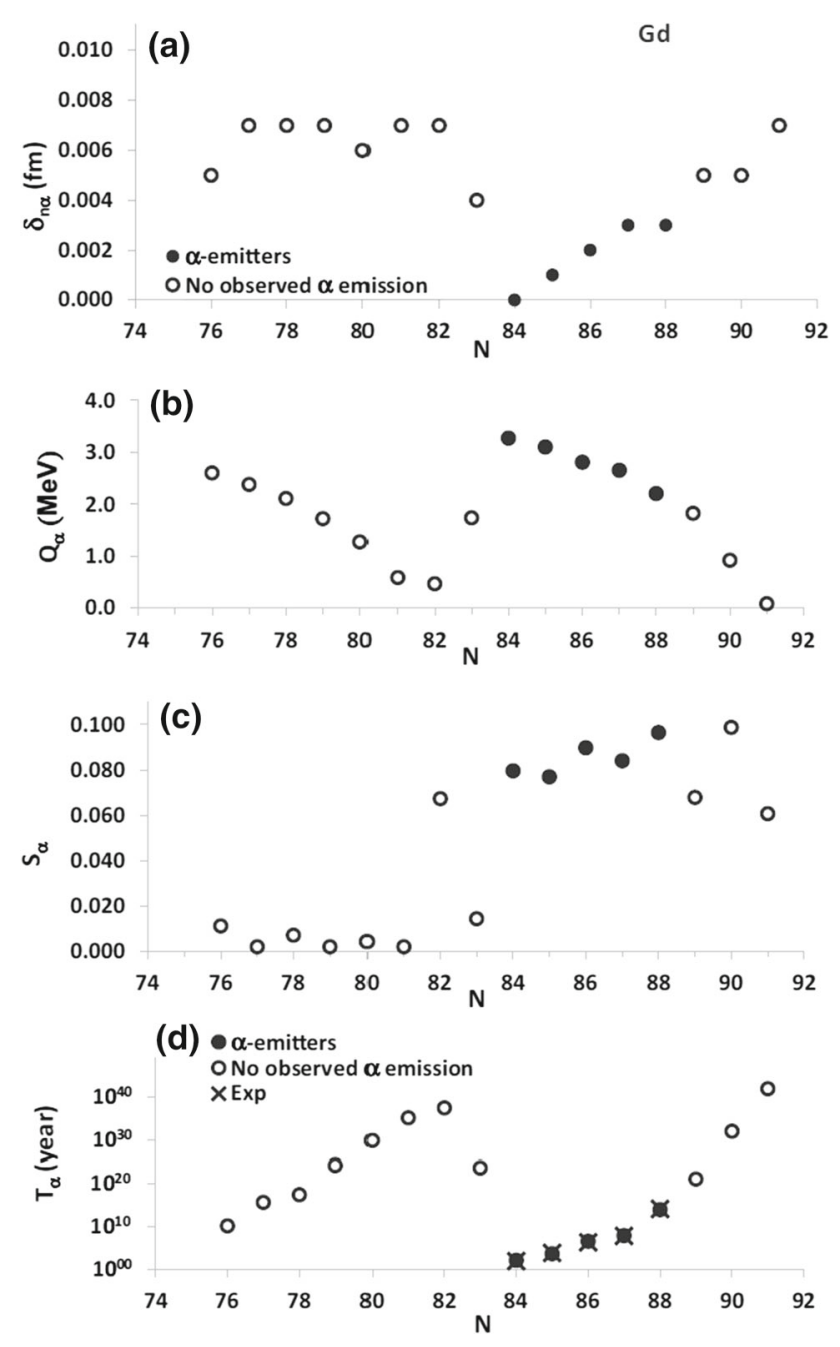

Fig. 54 a The difference $\delta_{n \alpha}(A, Z)=\Delta_{n}(A-4, Z-2)-\Delta_{n}(A, Z)$ between the neutron skin thickness in the ${ }^{140-155} \mathrm{Gd}$ isotopes and in their possible daughter $(A-4, Z-2)$ nuclei, as a function of neutron number $N$. b The $Q_{\alpha}$ values for the decays of the isotopes displayed. c The estimated $\alpha$-preformation probability. d The calculated partial half-lives, $T_{\alpha}$, for ${ }^{140-155} \mathrm{Gd}$. The observed partial half-lives $T_{\alpha}$ for ${ }^{148-152} \mathrm{Gd}$ are indicated in (d) by crosses

$Q_{\alpha}$ values. In ${ }^{148-152} \mathrm{Gd}$, the $\alpha$-emission has been observed. As seen in Fig. 54a, the $\alpha$-decay of these parent nuclei leads to a slight increase of the neutron skins in the daughter nuclei ${ }^{144-148} \mathrm{Sm}, \delta_{n \alpha}=0.000-0.003 \mathrm{fm}$. The increase in neutron skins of $(A-4, Z-2)$ nuclei corresponding to other isotopes ${ }^{140-147,153-155} \mathrm{Gd}$ exhibits larger values of $\delta_{n \alpha}$. Although the nuclei ${ }^{140-142} \mathrm{Gd}$ have $Q_{\alpha}$ (Fig. 54b) close to those for ${ }^{148-152} \mathrm{Gd}$ in which the $\alpha$-decays were observed and smaller neutron number $N$, the values of $\delta_{n \alpha}$ for them are relatively large and $\alpha$-decays are strongly hindered. Larger $Q_{\alpha}$ and minimal $\delta_{n \alpha}$ correlate with minimal $T_{\alpha}$ for the isotopic chain in Fig. 54, indicating the isotopes in which $\alpha$-decays can be easier detected. Comparing the results in Figs. 54a and d, we see that the observed $T_{\alpha}$ are almost proportional to the 
change $\delta_{n \alpha}$ of neutron skin thickness. The variation of the displayed half-lives with $N$ is very similar to that of $\delta_{n \alpha}$. The $\alpha$-decay of ${ }^{148} \mathrm{Gd}(N=84)$ to ${ }^{144} \mathrm{Sm}(N=82)$ with $\delta_{n \alpha}=0$ yields the shortest half-life of $\mathrm{Gd}$ isotopes. There is a clear tendency of the observed $\alpha$-decays to keep the neutron skin almost unchanged. Thus, both the large increase of the neutron skin difference and the slight decrease of the proton skin difference indicate the hindrance of the $\alpha$-decay.

The indicated correlation between $T_{\alpha}$ and the change of neutron (proton) skin thickness can be helpful in the study of neutron (proton) skins of radioactive nuclei [332]. It also offers a straightforward method to predict the shorter halflives against $\alpha$-decays for unknown heaviest nuclei by calculating their proton and neutron densities profiles. Some important estimates of the $\alpha$-decay characteristics can be obtained without the calculations of interaction potential and preformation factor [333], but rather calculating only the proton and neutron density profiles if the correlations between them and $\alpha$-decays are established in the isotopic chain. This might trigger further studies of nuclear properties with the self-consistent microscopic methods, which is of great importance for new radioactive beam facilities.

\section{EDF approach to predictions of the structure of SHN}

\subsection{Isotone chains of SHN}

The investigation of transfermium elements expands our knowledge of the single-particle structure, location of the shell closures, and decay modes [59,71-73,263,264,334, 335]. The spectroscopic study of low-lying one-quasiparticle states in the odd-mass actinides and transactinides [336-348] is up-to-date because of the problem of unambiguous identification of new superheavy nuclei $[4,349]$ as well as still unknown isotopes of heavy nuclei. Low-lying states have been identified in Es, Fm, Md, No, Lr, Rf, Db, and Bh isotopes [336-347]. For several of the heaviest nuclei, such spectroscopic information as single- and multi-particle excitations and rotational bands is now available. The experimental study of one-quasiparticle states in some of the $N=145,147,149$, 151 , and 153 nuclei has been performed.

The similarities in the $\alpha$-decay pattern as well as for excitation energies and ordering of low-lying Nilsson levels have been established for some isotone lines [337,344,345]. The analysis of isotonic chains of even-odd nuclei containing the transfermium nuclei would help us to assign the Nilsson configurations to the one-quasineutron states if one of the nucleus, usually with smaller $Z$, of this chain was studied in detail.

The decays of $K$ isomers in several nuclei with $Z \geq 99$ have been also studied [337]. The $\gamma$-decay of these isomers can populate the rotational structures. The isomers decaying by $\alpha$ emission are especially interesting if the $\alpha$-decay occurs into the same isomeric state of the daughter nucleus which can decay again by $\alpha$ emission. As known, in odd-mass heavy nuclei the $\alpha$-decays from the ground state preferably occurs into the same one-quasiparticle state of daughter nucleus.

To reveal the quasiparticle structure of the heaviest nuclei the large efforts were made both in experiments [336-348] and in theory [39,42,44,45,70-73,125,129,131, 263,264,334,335,350-359]. Existing semi-microscopical approaches [39,42,44,45,263,264,350,352,353] based on the Nilsson-Strutinsky method supply the basis for the intensive calculations of the properties of low-lying states of heavy nuclei. These approaches use the parametrization of nuclear shape and single-particle Hamiltonian. In Refs. [263,264], a large, seven-dimensional deformation space, $\left\{\beta_{\lambda}\right\}, \lambda=$ $2,3, \ldots, 8$, is used to obtain the equilibrium deformation of a nucleus. The contribution of an odd nucleon, occupying a single-particle state $\mu$, to the energy of a nucleus is described approximately by the one-quasiparticle energy $E_{\mu}$, calculated in the BCS approximation. Pairing interaction of the monopole type is taken. No blocking is used. The calculations have been performed for the isotones with $N=145-161$. Generally, the larger the excitation energy, the stronger the dependence on $Z$. In particular, the ground-state characteristics remain usually the same for rather long chain of isotones.

Besides these approaches, the self-consistent approaches $[125,129,131,357,358]$ have been also applied to calculate the structure of heavy nuclei. One can see that while some theoretical approaches describe better one group of nuclei, the other approaches are more adjusted for other nuclei. The uncertainty in the prediction of level order near the Fermi surface with different theoretical approaches lies in the uncertain strength of the spin-orbit coupling. The prediction of shell gaps and a realistic spectrum of low-lying excitations remains a challenge for the nuclear models, which have to be rigorously tested by a wide variety of the nuclear properties throughout the periodic table. New experimental results challenge modern theories attempting to reproduce the properties and structures of the heaviest nuclei. The comparison of calculated and experimental one-quasiparticle states allows us to test the parameters used in the theory and to assign the quantum numbers to the experimental levels, which is important in the analysis of $\gamma$ and $\alpha$ transitions.

The axially deformed SkHFB calculations have been performed by using the code described in Refs. [360,361]. The SLy4 [314,362] and SkP [182] parametrizations of the Skyrme interaction are chosen in the mean field (particlehole) channel. With these parametrizations one can describe well the spins and parities of the ground states of nuclei considered. In the pairing (particle-particle) channel, the zero-range density-dependent force was used. The LipkinNogami prescription is utilized to approximately restore the particle numbers. 


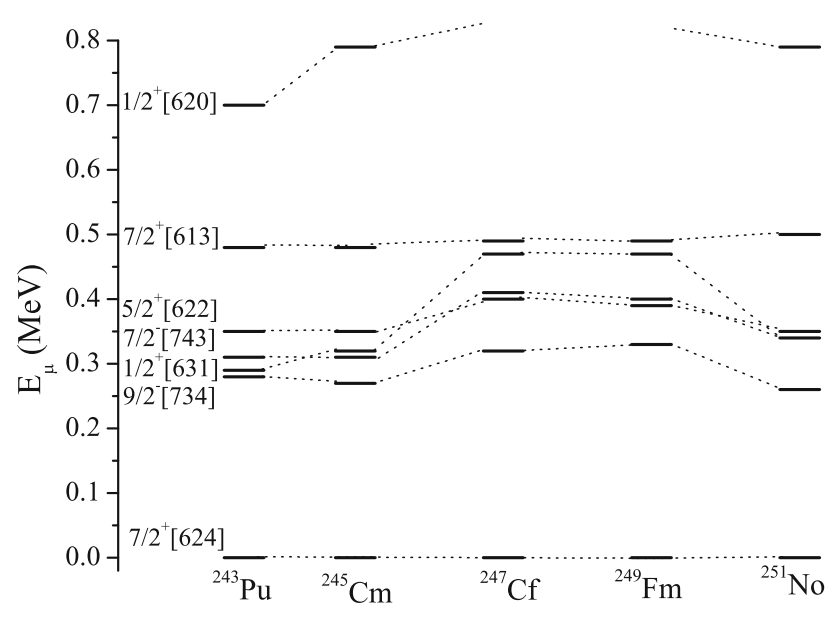

Fig. 55 Energies of low-lying one-quasineutron states in the $N=149$ nuclei calculated with the QPM

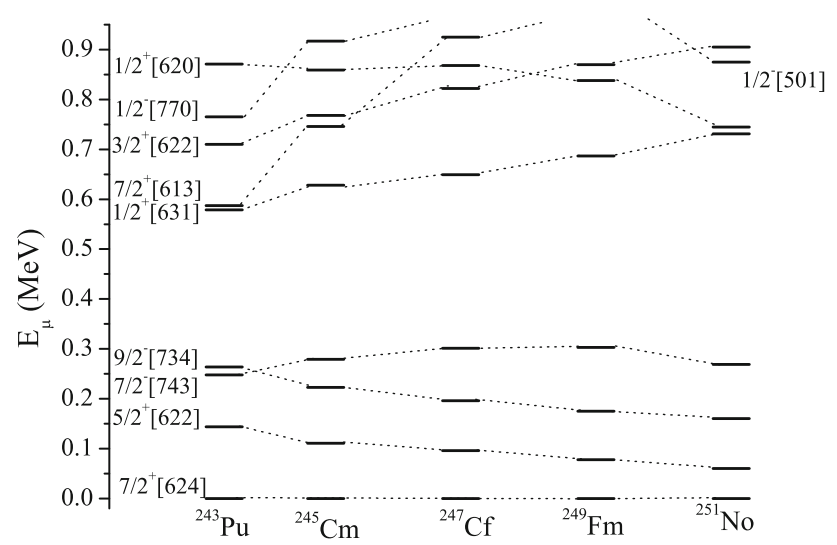

Fig. 56 Energies of low-lying one-quasineutron states in the $N=149$ nuclei calculated within the SkHFB approach using the SLy4 Skyrme interaction

For open shell nuclei, one has to deal with the occupation probabilities ranging from zero to one. Mathematically, this could be treated by introducing the concept of quasiparticles. In the framework of HFB, the quasiparticle operators are connected to the single-particle operators by a linear Bogoliubov transformation. The ground state is described as the quasiparticle vacuum. After a self-consistent procedure, the occupation probability and the quasiparticle energy $E_{\mu}$ for each level are determined by a transformation to the canonical basis. Quasiparticle excitations are fully self-consistently calculated. The effects of blocking of a pair of states are, thus, exactly taken into consideration.

\subsection{1 $N=149$ isotone chain}

In Figs. 55, 56 and 57 we show the one-quasiparticle spectra of the $N=149$ isotones calculated with the QPM and with the SkHFB approach using the SLy4 and SkP Skyrme forces. The description of firmly defined experimental lev-

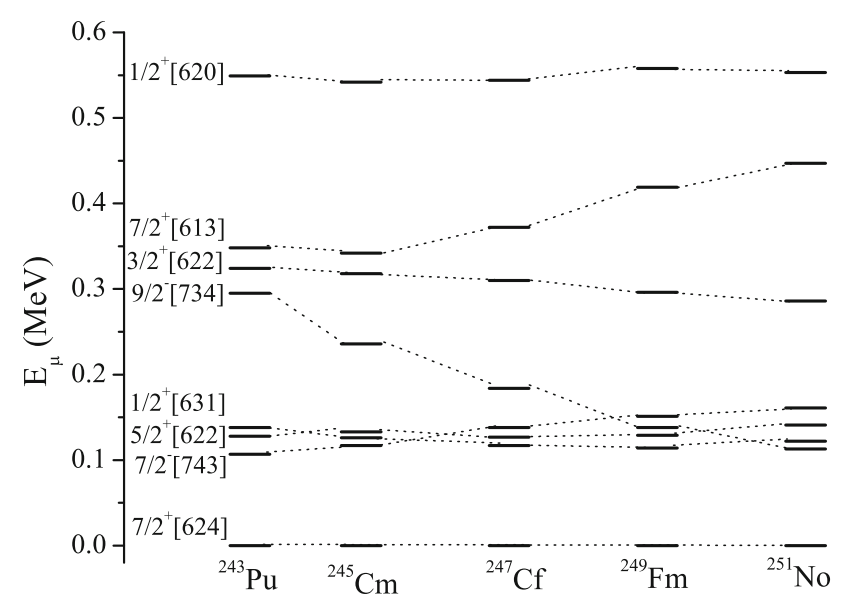

Fig. 57 The same as in Fig. 56, but for the SkP Skyrme interaction

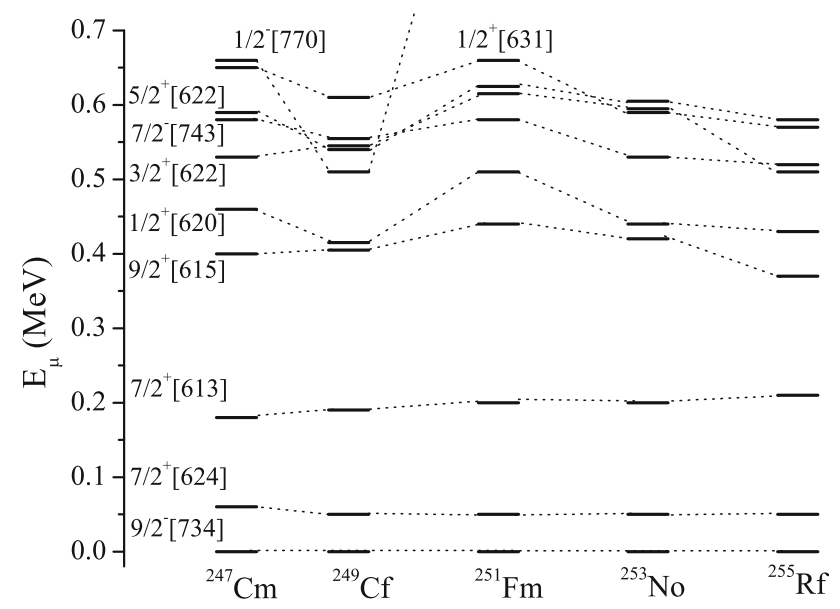

Fig. 58 Energies of low-lying one-quasineutron states in the $N=151$ nuclei calculated with the QPM

els is rather good, within $200 \mathrm{keV}$. The order of $5 / 2^{+}[622]$ and $1 / 2^{+}$[631] levels is well reproduced in Figs. 56 and 57 for ${ }^{243} \mathrm{Pu}$. The calculations with the QPM for ${ }^{243} \mathrm{Pu},{ }^{245} \mathrm{Cm}$, and ${ }^{251} \mathrm{No}$ (Fig. 55) result in an opposite order of these levels, which, however, are close in energy. In the SkHFB calculations with SLy4 parametrization the $1 / 2^{+}[631]$ state is well above the $5 / 2^{+}[622]$ state in all $N=149$ nuclei. The SkHFB calculation with the SkP parametrization leads to the close energies of the $5 / 2^{+}[622]$ and $1 / 2^{+}$[631] states. While the $1 / 2^{+}[631]$ state is slightly above the $5 / 2^{+}[622]$ state in ${ }^{243} \mathrm{Pu}$, the order becomes opposite in heavier nuclei in Fig. 55.

One can see the smooth change of energies of almost all one-quasiparticle states in the isotonic chain. Indeed, the deformation parameters of the $N=149$ nuclei treated are almost the same. In Fig. 57, the $1 / 2^{+}[631]$ level goes down with $Z$, but not as much as assumed in the experimental analysis [337]. 


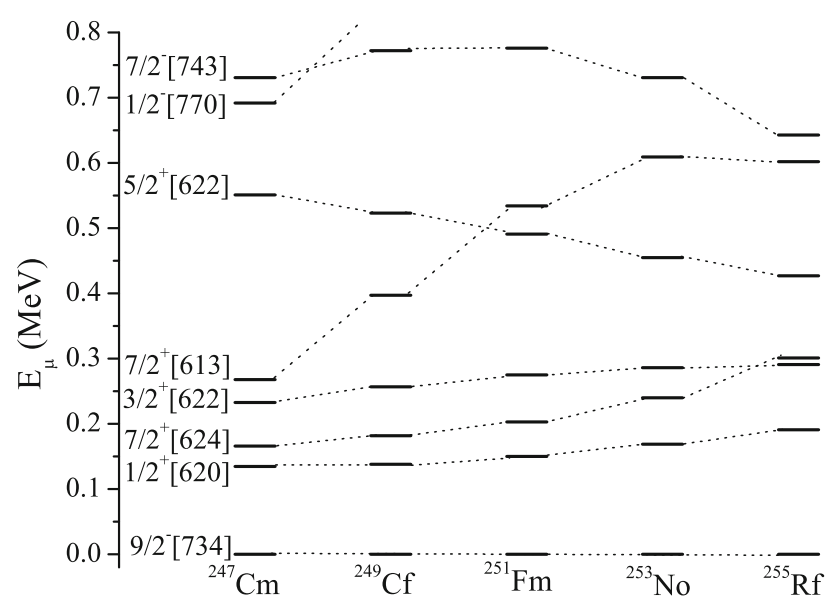

Fig. 59 Energies of low-lying one-quasineutron states in the $N=151$ nuclei calculated within the HFB approach using the SLy4 Skyrme interaction

\subsection{2 $N=151$ isotone chain}

For the $N=151$ nuclei, all the calculations with different methods (Figs. 58, 59 and 60) give the $1 / 2^{+}[620]$ state below the $5 / 2^{+}[622]$ state. In ${ }^{247} \mathrm{Cm}$, the opposite order of the $1 / 2^{+}$and $5 / 2^{+}$states is expected from the experiment [363]. This order is predicted for the $N=151$ nuclei in Refs. [263,264,353]. However, as in Figs. 58, 59 and 60 in Refs. [263,264,353] the $7 / 2^{+}[624]$ state is expected below the $5 / 2^{+}[622]$ state. This means that the $5 / 2^{+}[622]$ state cannot be the isomeric state because of the fast M1 transition into the $7 / 2^{+}$[624] level. In Figs. 58 and 59, the $1 / 2^{+}$[620] state is at energy larger than $0.15 \mathrm{MeV}$ and, thus, has sizeable admixture due to the interaction with phonons. This admixture decreases the lifetime resulting from Weisskopf estimate. The delayed $\gamma$ transition from $1 / 2^{+}$to $7 / 2^{+}$(Fig. 58) would follow by fast E1 transition to the ground state. The SkHFB approach with the SLy4 interaction results in the $1 / 2^{+}[620]$ to be the isomer in all $N=151$ nuclei (Fig. 59). Within the SkHFB approach with the SkP interaction, the one-quasiparticle spectrum is quite dense near the ground state (Fig. 60). The $1 / 2^{+}$[620] state becomes even the ground state for nuclei starting from ${ }^{251} \mathrm{Fm}$ in Fig. 60.

As follows from the calculations presented, in the $N=$ 149 and 151 nuclei the $1 / 2^{+}[620]$ single-particle state is above and the $5 / 2^{+}[622]$ and $1 / 2^{+}$[631] single-particle states are below the corresponding Fermi levels. These levels go down with deformation. If in ${ }^{251}$ No one adjusts the $1 / 2^{+}[631]$ level closer to the Fermi level than the $5 / 2^{+}$[622] level, then in ${ }^{253}$ No the $1 / 2^{+}$[631] level would be below or just above the $5 / 2^{+}[622]$ level, if the cross of these levels occurs near the ground state. Thus, one cannot simultaneously adjust the calculated one-quasiparticle states to the experimental assignments for ${ }^{251} \mathrm{No}$ and ${ }^{253}$ No [337]. This statement is true if the deformations of ${ }^{251} \mathrm{No}$ and ${ }^{253} \mathrm{No}$

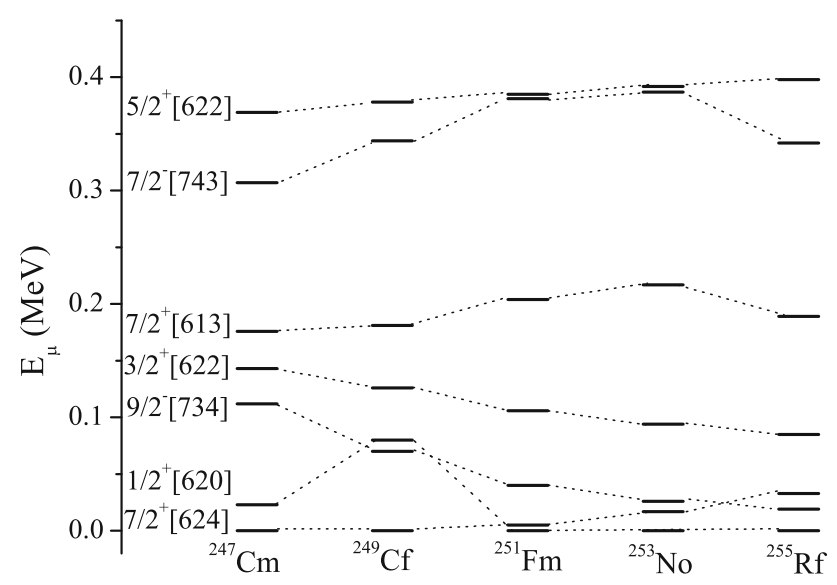

Fig. 60 The same as in Fig. 59, but for the SkP Skyrme interaction

are close, as follows from the calculations. Therefore, with reasonable variation of the parameters one can improve the agreement with the data on the $N=149$ nuclei but then one makes worse the description of the $N=151$ nuclei. In the isotone nuclei a strong change, of the order of quasiparticle levels, occurs if the proton number becomes magic or semi-magic and the nuclear deformation visibly changes. Otherwise the one-quasiparticle levels of isotonic nuclei vary within a small interval of the energy.

\subsection{Odd-Odd SHN}

The structure of superheavies crucially influences the evaporation residue cross-sections in the actinide-based complete fusion reactions [204]. Although the low cross-sections for the production of the superheavy nuclei offer rather restricted nuclear-structure information, in recent years the set of experimental data on the structure of the heaviest nuclei has been considerably increased because the experimental setups began to combine $\alpha-, e^{-}$-, and $\gamma$-spectroscopy $[337,364]$. In the experimental study [20], the $\alpha$-decay chains of the ${ }^{288} \mathrm{Mc}$ nucleus were produced in the ${ }^{243} \mathrm{Am}+{ }^{48} \mathrm{Ca}$ reaction. Structure information on the low-lying states of the odd-odd superheavy nuclei below ${ }^{288} \mathrm{Mc}$ was obtained in $\alpha-\gamma$ coincidences. The E1 transitions in ${ }^{276} \mathrm{Mt}$ were observed. These transitions represent a demanding test for theoretical models and so require a comprehensive analysis.

In Ref. [365], the shell structure of odd-even nuclei in the $\alpha$-decay chain of ${ }^{287} \mathrm{Mc}$ was studied within the selfconsistent SkHFB approach and the macroscopic-microscopic Nilsson-Strutinsky model to reveal possible E1 transitions in involved nuclei. In the SkHFB, the global UNEDF1 [366] and locally optimized UNEDF1 ${ }^{\text {SO }}$ [367] parametrizations were used. A variety of scenarios of E1 transitions in oddodd ${ }^{276}$ Mt were suggested. These scenarios were shown to be model-dependent: different models and parametrizations did not offer a unique explanation of the E1 transitions. 
Table 4 Equilibrium quadrupole deformations $\left(\beta_{2}\right)$ of nuclei belonging to the $\alpha$-decay chain of the nucleus ${ }^{286} \mathrm{Mc}$ calculated using the SkHF approach

\begin{tabular}{lcccccc}
\hline Nucleus & ${ }^{286} \mathrm{Mc}$ & ${ }^{284} \mathrm{Nh}$ & ${ }^{280} \mathrm{Rg}$ & ${ }^{276} \mathrm{Mt}$ & ${ }^{272} \mathrm{Bh}$ & ${ }^{268} \mathrm{Db}$ \\
\hline$\beta_{2}$ & 0.145 & 0.165 & 0.20 & 0.24 & 0.26 & 0.26 \\
\hline
\end{tabular}

In this connection, an additional analysis of the theoretical approaches is desirable.

To calculate the quasiparticle structure, one can use the potential obtained self-consistently with the Skyrme interaction realized with the parametrization SV-bas [368]. This parametrization represents a recent family of Skyrme forces [368] obtained with a systematic fit to nuclear matter features and a number of nuclear observables, including neutron skin and superheavy elements. What is important for our aims is that the fit of these forces involved binding energies and $Q_{\alpha}$ values in heavy and superheavy nuclei. The calculations were performed with the code SKYAX [369]. The equilibrium deformations were determined by the minimization of the total energy of the system. The pairing uses the surface (density-dependent) $\delta$-force $[313,370,371]$ at the BCS level.

The nuclear excitations were calculated in approximation of independent quasiparticles. Following our estimations, the differences in the one-quasiparticle spectra obtained with different potentials exceed a possible effect of the residual proton-neutron interaction. Thus, no residual interaction is used. The same level of the theoretical treatment (singleparticle potential + pairing) was used in Ref. [365]. Taking into account the present theoretical and experimental status of spectroscopy of SHN, this level of the analysis looks quite relevant. At least, it constitutes a first and necessary step paving the way for further progress.

The calculated equilibrium quadrupole deformations of nuclei belonging to the $\alpha$-decay chain of ${ }^{288} \mathrm{Mc}$ are listed in Table 4. As seen, the single-particle potentials used result in a smooth increase of $\beta_{2}$ along the $\alpha$-decay chain. The value of the equilibrium quadrupole deformation in ${ }^{288} \mathrm{Mc}$ and ${ }^{284} \mathrm{Nh}$ is roughly the same as an amplitude of the zero-point $\beta_{2}$ fluctuations in the typical spherical nuclei. However, the calculations have shown that the depths of the minima are sufficiently large, namely, from 3 to $4 \mathrm{MeV}$. It is interesting that the depths of the minima corresponding to prolate and oblate shapes almost coincide. Their difference is about $200 \mathrm{keV}$. This could mean that the isotopes around $Z=114$ are $\gamma$-soft. This fact can influence the $\alpha$-decay probabilities if the transitions occur from $\gamma$-soft to well deformed nuclei along the $\alpha$-decay chain of ${ }^{288} \mathrm{Mc}$.

The one-quasiproton and one-quasineutron spectra are presented in Figs. 61 and 62 for nuclei of the $\alpha$-decay chain of ${ }^{288} \mathrm{Mc}$. An interesting characteristic of the singleparticle spectra of the deformed nuclei along the $\alpha$-decay chain of ${ }^{288} \mathrm{Mc}$ is a splitting of the pseudospin doublets.
The reader is referred to Ref. [136] for a recent review on the pseudospin symmetry in atomic nuclei. There are four doublets located near the Fermi surface in the nuclei considered. Namely, two neutron doublets 5/2[613]-3/2[611], 11/2[606]-9/2[604] and two proton doublets 7/2[503]9/2[505], 1/2[510]-3/2[512]. In the single-particle scheme presented in Ref. [38], the splitting of this doublet is approximately equal to $650 \mathrm{keV}$. For the proton doublets 7/2[503]9/2[505] and 1/2[510]-3/2[512], Ref. [38] results in the splitting of $835220 \mathrm{keV}$, respectively. The splitting of the neutron pseudospin doublets obtained in the Skyrme approach is rather small: $80-200 \mathrm{keV}$ for the neutron doublet $5 / 2[613]-3 / 2[611]$ and $150-840 \mathrm{keV}$ for the neutron doublet 11/2[606]-9/2[604]. However, the splitting of the proton pseudospin doublets obtained with the Skyrme meanfield potential is quite large: $1.4-1.8 \mathrm{MeV}$ for $p 7 / 2[503]$ $p 9 / 2[505]$ and 300-500 keV for $p 1 / 2[510]-p 3 / 2[512] \mathrm{dou}-$ blets.

If we compare the spectra of the low-lying one-quasiparticle states produced by different single-particle potentials, we find some similarities. However, the differences in the spectra of the low-lying one-quasineutron states are rather significant. As expected, the one-quasiparticle spectra are denser near the ground state in the case of the phenomenological single-particle potentials which do not use any reduction of the effective nucleon mass.

Taking into account a possibility of the E1, E2, M1, and M2 transitions between the excited states of nuclei shown in Figs. 63 and 64 whose half-lifetimes are shorter than the characteristic times of the $\alpha$-transitions and keeping only $\alpha$ transitions with shortest lifetimes, the $\alpha$-decay spectra and possible $\gamma$-transitions are analyzed in the nuclei of $\alpha$-decay chain of ${ }^{288} \mathrm{Mc}$. The most probable calculated $Q_{\alpha}$ values are shown in Figs. 63 and 64 together with the available experimental data. The transitions keeping the quantum numbers of the odd proton and odd neutron are mainly taken into account. Using the Weisskopf estimate and the selection rules for the asymptotic quantum numbers [39], one can calculate the halflives $T_{\gamma}$ for the $\gamma$-transitions between the quasiparticle states.

The Skyrme-based calculations provide us with the nuclear binding energies, quasiparticle spectra and $Q_{\alpha}$ values for the ground-state-ground-state $\alpha$-decays. Besides the ground state $n 1 / 2[611] \otimes p 1 / 2[510]$, the possible isomeric states $n 1 / 2[611] \otimes p 7 / 2[503]$ and $n 15 / 2[707] \otimes p 1 / 2[510]$ can be populated in formation of ${ }^{288} \mathrm{Mc}$. Taking the calculated value of $Q_{\alpha}$ for the ground-state-ground-state $\alpha$-decay, the $\alpha$-decay from these states to the states with the same quantum numbers in ${ }^{284} \mathrm{Nh}$ would have energies $Q_{\alpha}=10.79$, 10.59 , and $10.54 \mathrm{MeV}$, respectively, which is in good agreement with available experimental data $[6,20]$. The scheme of $\alpha$-decay of the evaporation residue ${ }^{288} 115$ is presented in Fig. 65. After the M2, M1, and E2 gamma-transitions following the $\alpha$-decay with $Q_{\alpha}=10.54 \mathrm{MeV}$, and the M1 
Fig. 61 The calculated one-quasiproton spectra calculated with the SkHFB approach for the nuclei of the $\alpha$-decay chain of ${ }^{288} \mathrm{Mc}$. The Nilsson asymptotic quantum numbers are indicated

Fig. 62 The same as in Fig. 61, but for the one-quasineutron spectra

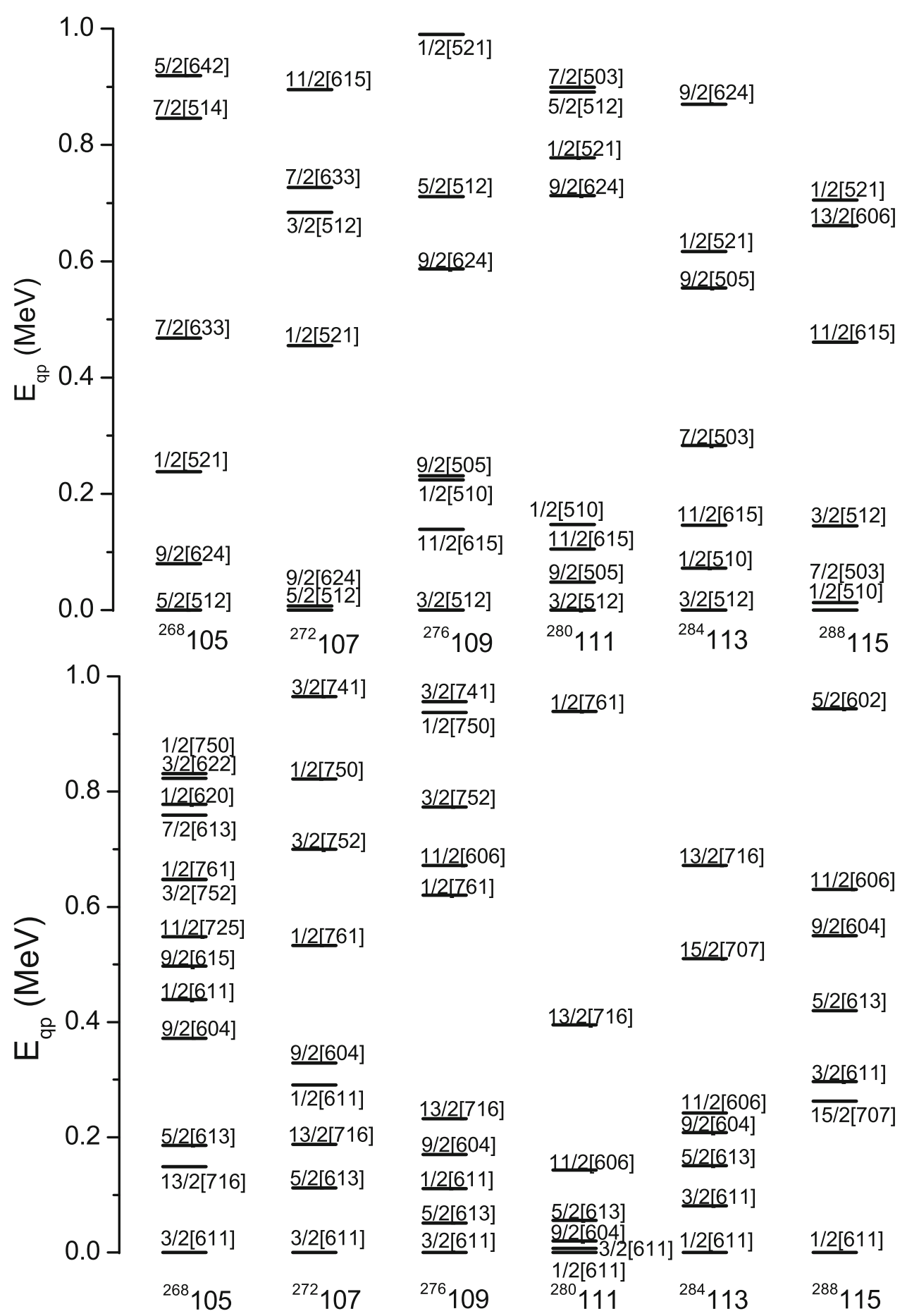

isomeric states should be rather long to observe $\alpha$-decays from them. Thus, in the self-consistent approach considered here the $\alpha$-decay of ${ }^{288} \mathrm{Mc}$ mainly follows by M1 and M2 transitions. Note that the E2 transitions are expected to be strongly suppressed between the members of the pseudospin doublets $n 11 / 2$ [606]- $n 9 / 2$ [604] and $p 1 / 2[510]-p 3 / 2[512$ ] [372]. With $Q_{\alpha}=10.61$ and $10.65 \mathrm{MeV}$ the $\alpha$-decays from the mentioned states of ${ }^{284} \mathrm{Nh}$ populate the states with 


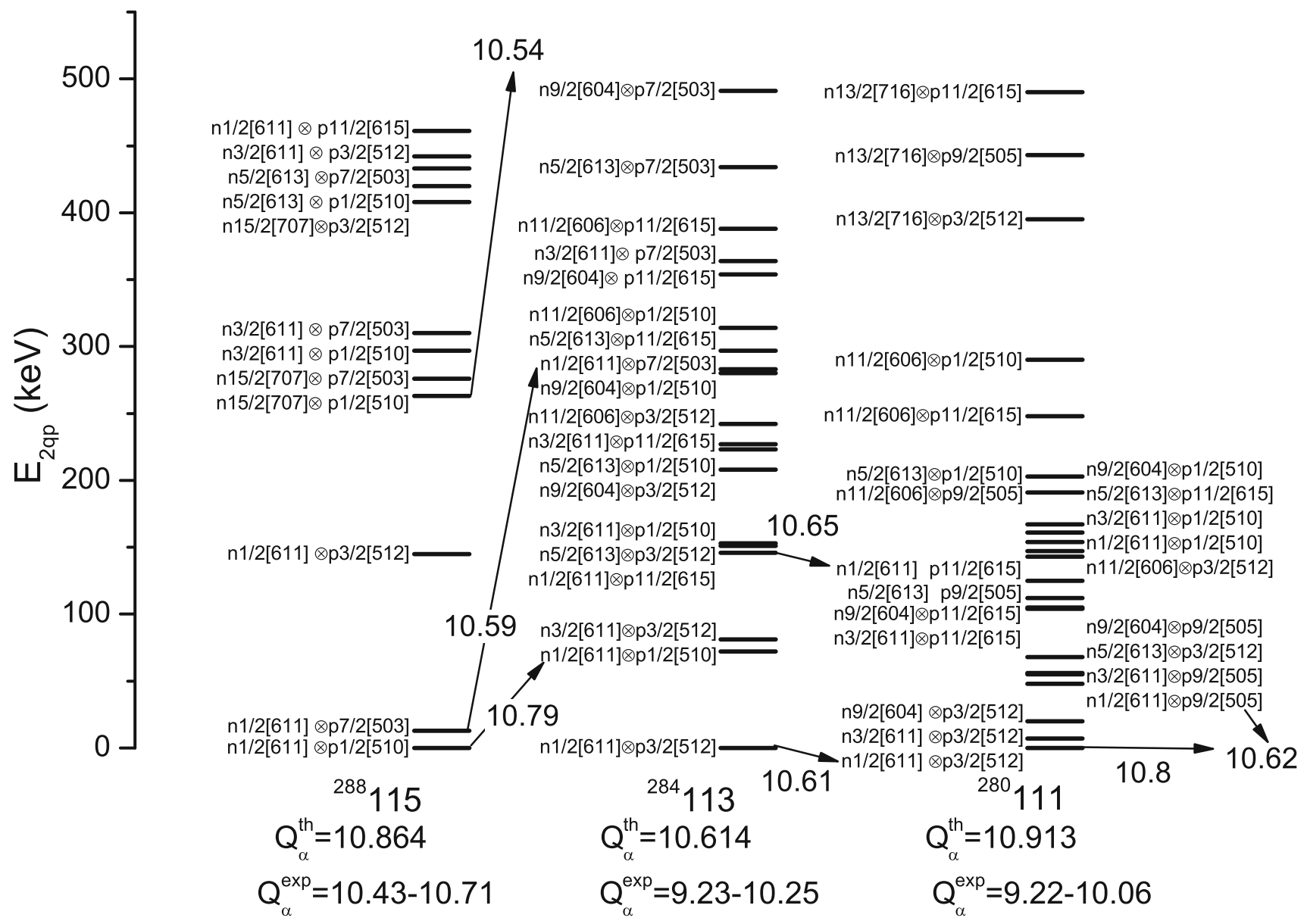

Fig. 63 The low-lying two-quasiparticle spectra calculated with the SkHFB approach for the nuclei of the $\alpha$-decay chain of ${ }^{288} \mathrm{Mc}$. The possible $\alpha$-decays with $Q_{\alpha}$-values are indicated. The calculated $Q$ th $_{\alpha^{-}}$

the same quantum numbers in the daughter nucleus ${ }^{280} \mathrm{Rg}$ (Fig. 66).

\section{EDF approach to $\beta$-decay and EC}

\subsection{Heavy actinides and SHN}

The evaporation of proton or $\alpha$-particle from compound nucleus, produced in the hot-fusion reactions, leads to the formation of nuclei with smaller $Z$ but with larger neutron excess. In addition, the electron capture (EC) can occur by converting a proton into a neutron to the daughter nucleus. Therefore, it is of great interest to study the competition between $\beta^{+} / \mathrm{EC}$ and $\alpha$-decays in SHN produced in the $p x n$ and $\alpha x n$ evaporation channels of hot-fusion reactions. Also, the $\beta^{+}$and EC branches would open the possibility to reach other SHN not belonging to the original $\alpha$-decay chains. These new branches would be open if the $\beta^{+} / \mathrm{EC}$ and $\alpha$ decay half-lives are comparable [373]. values for the ground-state-ground-state $\alpha$-decays as well as the available experimental values of $Q_{\alpha}^{\exp }$ are presented

In the calculations of the $\beta^{+} / \mathrm{EC}$ half-lives there are three main ingredients: (1) the corresponding $Q_{E C}$ energies, which are taken from experiment when available or from calculations in other cases; (2) the phase-space factors for each transition, which are calculated in a similar way in practically all the existing calculations of the half-lives; and (3) the nuclear structure that generates the energy distribution of the GT strength. In Refs. [374,375], this distribution is taken from the microscopic calculations based on the Skyrme effective nucleon-nucleon interactions. The solution of the HF equations is found by using the formalism developed in Ref. [78,376], assuming time reversal and axial symmetry. Figure 67 shows the ratios of the calculated half-lives to the experimental ones for some $\mathrm{Fm}, \mathrm{Md}$, and No isotopes, where there are experimental data. The results are obtained with the experimental $Q_{E C}$ values, but for different shapes that include the ground state $\left(\beta_{2} \approx 0.3\right)$ as well as the oblate $\left(\beta_{2} \approx-0.3\right)$ and superdeformed prolate $\left(\beta_{2} \approx 0.7\right)$ shapes. The uncertainties on the half-lives related to the $Q_{E C}$ energies, $J^{\pi}$ assignments, and nuclear shapes are comparable, 
Fig. 64 The continuation of Fig. 63

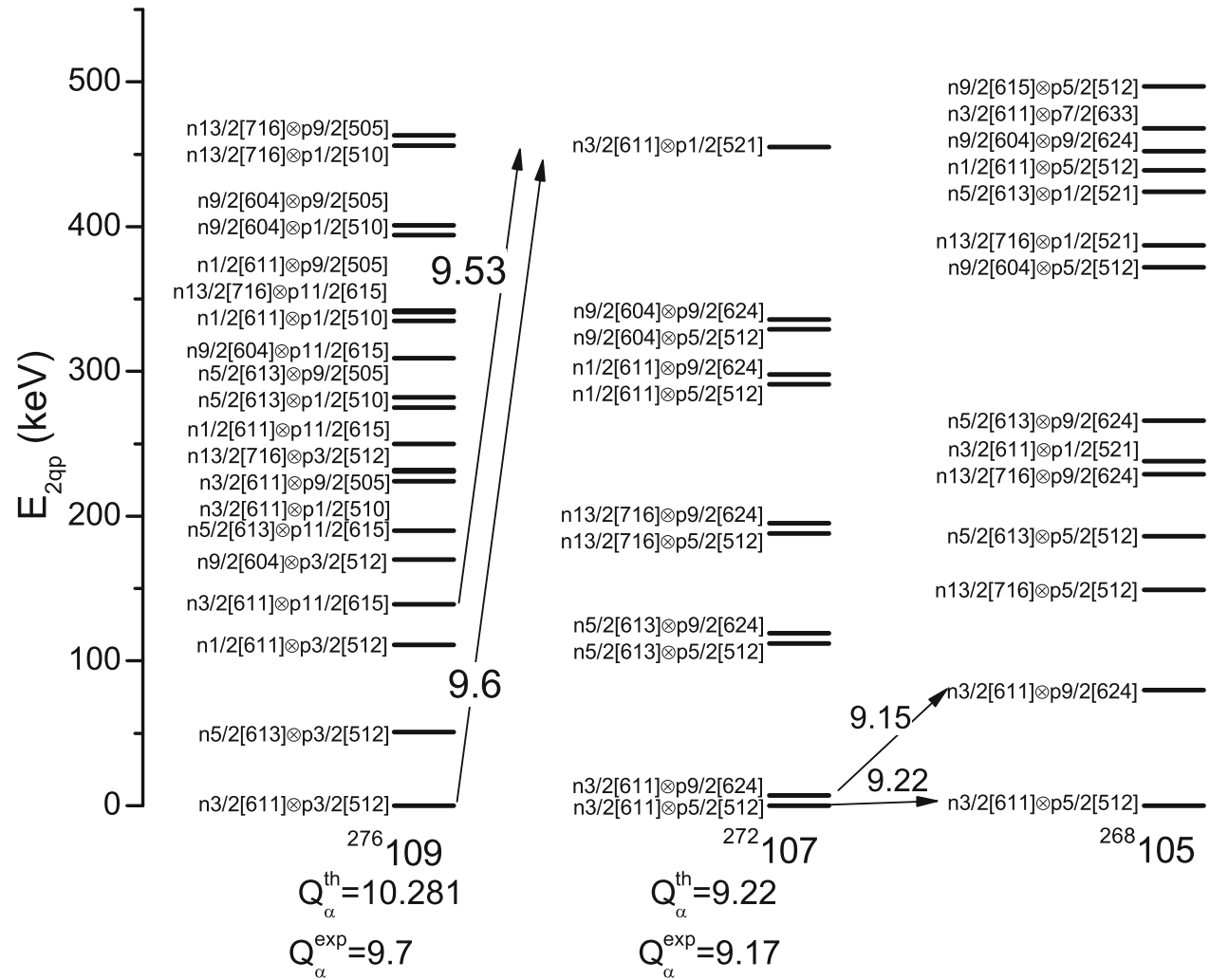

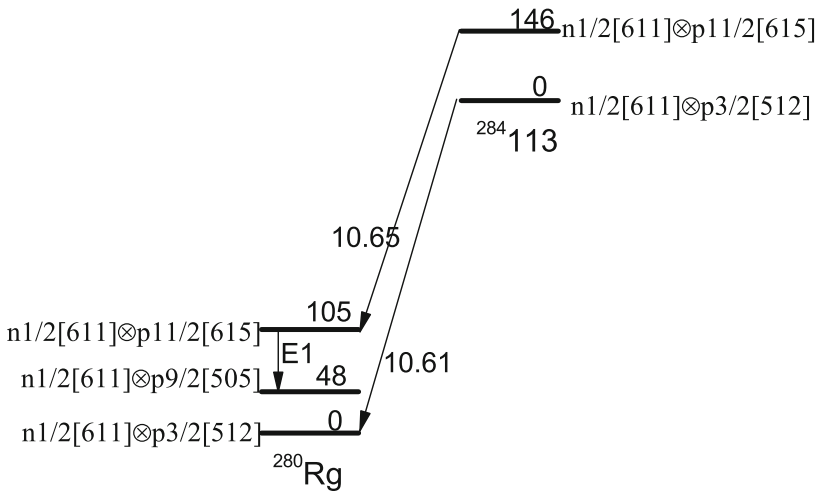

Fig. 66 The same as in Fig. 65, but for the decay ${ }^{284} \mathrm{Nh} \rightarrow{ }^{280} \mathrm{Rg}$

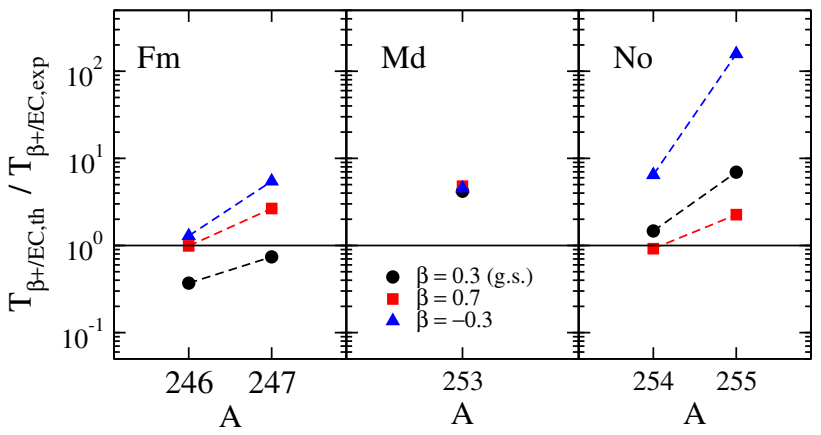

Fig. 67 Ratios of calculated and experimental half-lives for indicated Fm, Md, and No isotopes [374]. The results are obtained with experimental $Q_{E C}$ energies for the three shapes that produce energy minima on the potential energy surface 


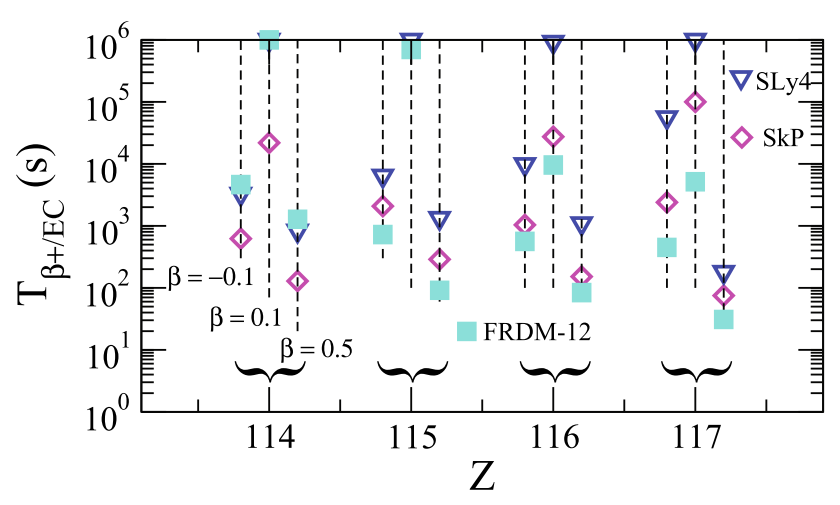

Fig. 68 Calculated half-lives, $T_{\beta^{+}} / E C$ (s) for ${ }^{290} \mathrm{Fl},{ }^{293} \mathrm{Mc},{ }^{294} \mathrm{Lv}$, and ${ }^{295}$ Ts. Half-lives for the ground-state oblate $\beta_{2}=-0.1$ (left vertical lines), prolate $\beta_{2}=0.1$ (middle vertical lines), and prolate $\beta_{2}=0.5$ (right vertical lines) nuclear shapes obtained from the indicated mass formulas to calculate $Q_{E C}$ [374]

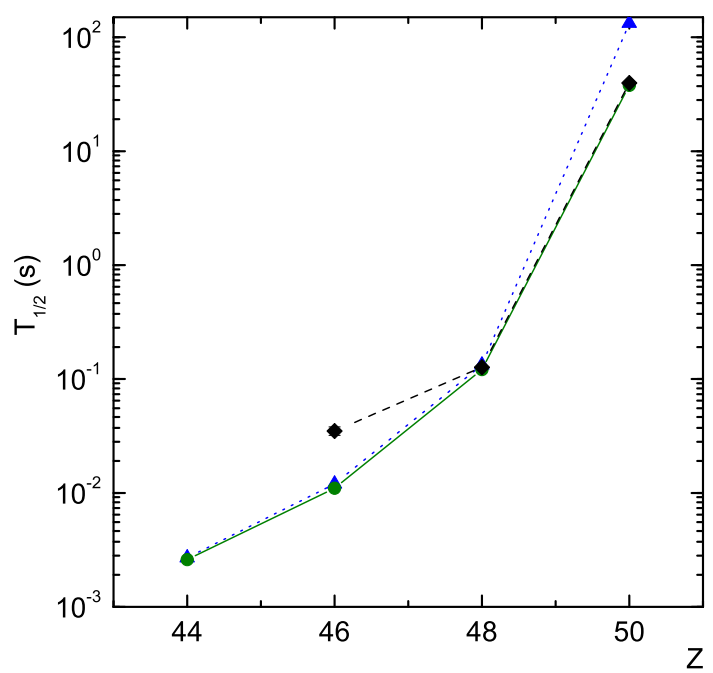

Fig. 69 The phonon-phonon coupling effect on $\beta$-decay half-lives of the neutron-rich $N=82$ isotones [378]. The circles correspond to the half-lives calculated with inclusion of the $\left[1_{i}^{+} \otimes 2_{i^{\prime}}^{+}\right]_{\mathrm{QRPA}}$ configurations; the triangles are the QRPA calculations. Experimental data (diamonds) are from Refs. [379,380]

\section{$9.2 \beta$-decay half-lives and $\beta$-delayed multi-neutron emission in heavy nuclei}

The nuclear $\beta$-decay properties are important for understanding the nuclear-structure evolution at extreme $N / Z$ ratios, for analysis of radioactive ion-beam experiments, and modeling of the rapid neutron-capture process (in astrophysics nucleosynthesis by the $r$-process) [377,378]. The $\beta$-decay of "waiting-point nuclei" ${ }^{129} \mathrm{Ag},{ }^{130} \mathrm{Cd},{ }^{131} \mathrm{In}$ and $\beta$-delayed multi-neutron emission $(\beta x n, x=1,2,3,4, \ldots)$ have recently attracted a lot of experimental and theoretical efforts [379-388]. The $\beta x n$-process depends on the $\beta$ decay energy release, neutron-emission thresholds, and $\beta$ strength function (the spectral distribution of $\beta$-decay matrix elements within the $\beta$-decay window). An adequate descrip-
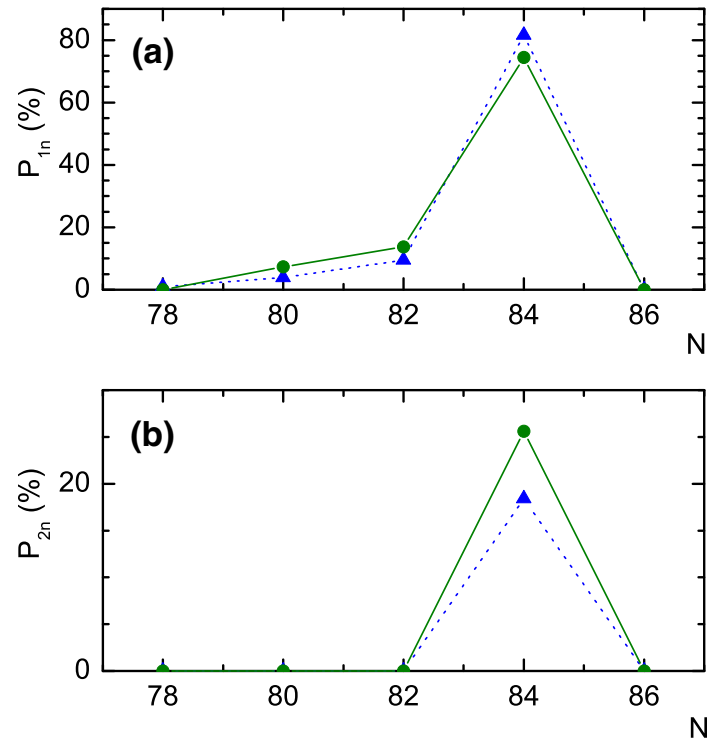

Fig. 70 The phonon-phonon coupling effect on $\beta$-delayed neutronemission probabilities of the neutron-rich Cd isotopes. The circles correspond to the probabilities calculated with inclusion of the $\left[1_{i}^{+} \otimes 2_{i^{\prime}}^{+}\right]_{\mathrm{QRPA}}$ configurations; the triangles are the QRPA calculations [378]

tion of the differential quantities poses constraints on the EDF in the high-isospin-asymmetry regime. Assuming an amplification of the intensity distribution by the integral Fermi factor, the most important contributions come from the allowed Gamow-Teller and high-energy first-forbidden $\beta$ decays. Importantly, they should be treated by employing the one and the same self-consistent framework [378,383,384]. Using the SkEDF T43 with the tensor components and the surface-peaked zero-range pairing interaction, the halflives and multi-neutron-emission probabilities are analyzed in Ref. [378]. The finite-rank separable approximation is employed in order to handle very large two-quasiparticle spaces. As seen in Fig. 69, the QRPA calculations give a reasonable agreement with experimental data for the $\beta$-decay half-life of the neutron-rich doubly magic nucleus ${ }^{132} \mathrm{Sn}$. Accounting for the phonon-phonon coupling effect improves the description and reproduces the 313 -fold reduction of $T_{1 / 2}$ from ${ }^{132} \mathrm{Sn}$ to ${ }^{130} \mathrm{Cd}$. A successful description of the experimental neutron-emission probabilities is first obtained in the framework of the Skyrme interaction (Fig. 70) [378]. Significant two-neutron emission for ${ }^{132} \mathrm{Cd}$ is predicted. A nonzero probability of the neutron emission is found for ${ }^{126} \mathrm{Cd}$. The coupling between one- and two-phonon configurations in the wave functions of $1^{+}$states is shown to be essential. The QRPA underestimates the ratio $P_{2 n} / P_{1 n}$. Inclusion of the two-phonon configurations produces an impact on the $P_{2 n}$ value which leads to an $55 \%$ increase of the ratio $P_{2 n} / P_{1 n}$. Further improvement of this model can be carried out by improving the isospin dependence in the EDF. 
9.3 EC cross-sections for neutron-rich $N=50$ nuclei in massive stars

The EC on nuclei reduces the electron-to-baryon ratio $Y_{e}$ and hence pressure and initiates the gravitational collapse of the core of a massive star triggering a supernova explosion $[377,389]$. As the neutrinos produced by EC are carrying away energy from a star, it is also an effective cooling mechanism, resulting in the fact that heavy nuclei survive in the collapse process $[377,389]$. The decrease of the $Y_{e}$ ratio leads to the abundance distribution of nuclei shifted to more neutron-rich and heavier isotopes. The EC in stellar environments is considered in Refs. [390,391] under the following assumptions: (1) The temperature in the stellar interior is sufficiently high (a few hundred $\mathrm{keV}$ to a few $\mathrm{MeV}$ ) and atoms are fully ionized, and the surrounding electron gas is described by the Fermi-Dirac distribution, with temperature and chemical potential. Neutrinos escape freely from the star. Hence no Pauli blocking for neutrinos is considered in the final state. (2) The parent nucleus is in a thermal equilibrium state treated as the thermal (phonon) vacuum. (3) The EC leads to charge-exchange transitions from the thermal vacuum to thermal one-phonon states. In Ref. [391], the proton-neutron quasiparticle RPA with the Skyrme interaction $\left(\mathrm{SkM}^{*}, \mathrm{SkO}^{\prime}\right)$ is extended to finite temperature by the thermofield dynamics formalism. This approach allows us to calculate the stellar weak-interaction processes at finite temperature in a thermodynamically consistent way. The energies of the GT transitions from the nuclear ground and thermally excited states and corresponding transition strengths are calculated as functions of the nuclear temperature. This technique does not rely on Brink's hypothesis. Moreover, the Ikeda sum rule is proven to be fulfilled at finite temperature. For the nuclei ${ }^{78} \mathrm{Ni},{ }^{82} \mathrm{Ge},{ }^{86} \mathrm{Kr},{ }^{88} \mathrm{Sr}$, one can observe in Fig. 71 [391] a similar evolution of the EC crosssections with increasing temperature, reflecting the temperature dependence of the $\mathrm{GT}_{+}$strength distributions [391]. For all nuclei, the ground-state $\mathrm{GT}_{+}$distributions have no strength at low energies $(<7 \mathrm{MeV})$, in agreement with the Pauli blocking of the allowed GT $\left(\mathrm{GT}_{+}\right)$strength due to the $N=50$ shell gap. Once the threshold for the EC is overcome, the cross-sections increase with electron energies, where the energy dependence is mainly dictated by the phase space. With increasing temperature, the $\mathrm{GT}_{+}$strength is shifted towards lower energies, reducing the gap which has to be overcome or even removing it completely if the strength is shifted to negative energies due to downscattering transitions [391]. The EC rates at temperature $0.86 \mathrm{MeV}$ are shown in Fig. 72 [391] for nuclei indicated at temper. The EC rates for ${ }^{86} \mathrm{Kr}$ and ${ }^{88} \mathrm{Sr}$ derived with the ground-state QRPA calculations are consistent with those derived from the experimental data. The rates at finite temperature are significantly larger than those at zero temperature, stressing the

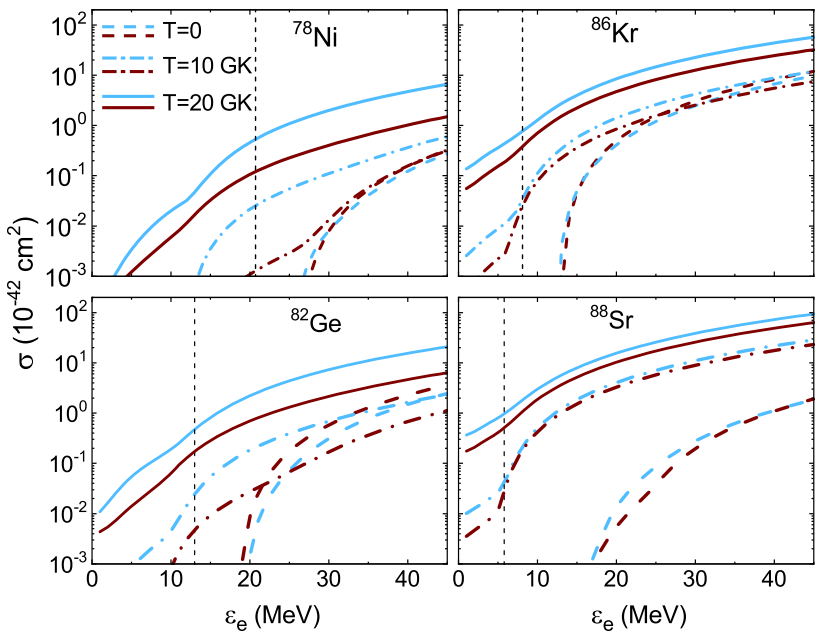

Fig. 71 EC cross-sections for ${ }^{78} \mathrm{Ni},{ }^{82} \mathrm{Ge},{ }^{86} \mathrm{Kr}$, and ${ }^{88} \mathrm{Sr}$ at temperature $T=0,10,20 \mathrm{GK}$. The blue (light gray) and brown (dark gray) curves represent results obtained with the $\mathrm{SkM}^{*}$ and $\mathrm{SkO}^{\prime}$ interactions, respectively. The dashed vertical lines indicate the ground-state thresholds $Q=M_{f}-M_{i}$

importance of thermal unblocking effects. While the EC is dominated by $\mathrm{GT}_{+}$at low densities (the early stage of the collapse), the contributions from forbidden transitions become increasingly relevant with growing densities at the later collapse phases. The thermal unblocking (due to the thermal excitations) and the contributions from forbidden transitions enhance the capture rates by an order of magnitude or even higher. These results also indicate that the $N=50$ shell gap does not act as an obstacle for EC in the later collapse stage. Within this approach, it would be desirable to perform also calculations with other realistic effective interactions.

\section{Fission and cluster radioactivity in actinides and SHN}

\subsection{Fission barriers}

As detailed in Sect. 5.5, the height of the fission barrier, together with the neutron separation energy, determines the survival probability of the excited compound nucleus formed in the heavy-ion fusion reaction for synthesizing SHN. The fission is a typical large scale nuclear collective motion involving various shape degrees of freedom among which the most relevant one is the axial quadrupole deformation $\beta_{20}$ in the following parametrization of the nuclear surface in terms of a multipole expansion:

$R(\theta, \varphi)=R_{0}\left[1+\beta_{00}+\sum_{\lambda=1}^{\infty} \sum_{\mu=-\lambda}^{\lambda} \beta_{\lambda \mu}^{*} Y_{\lambda \mu}(\theta, \varphi)\right]$ 


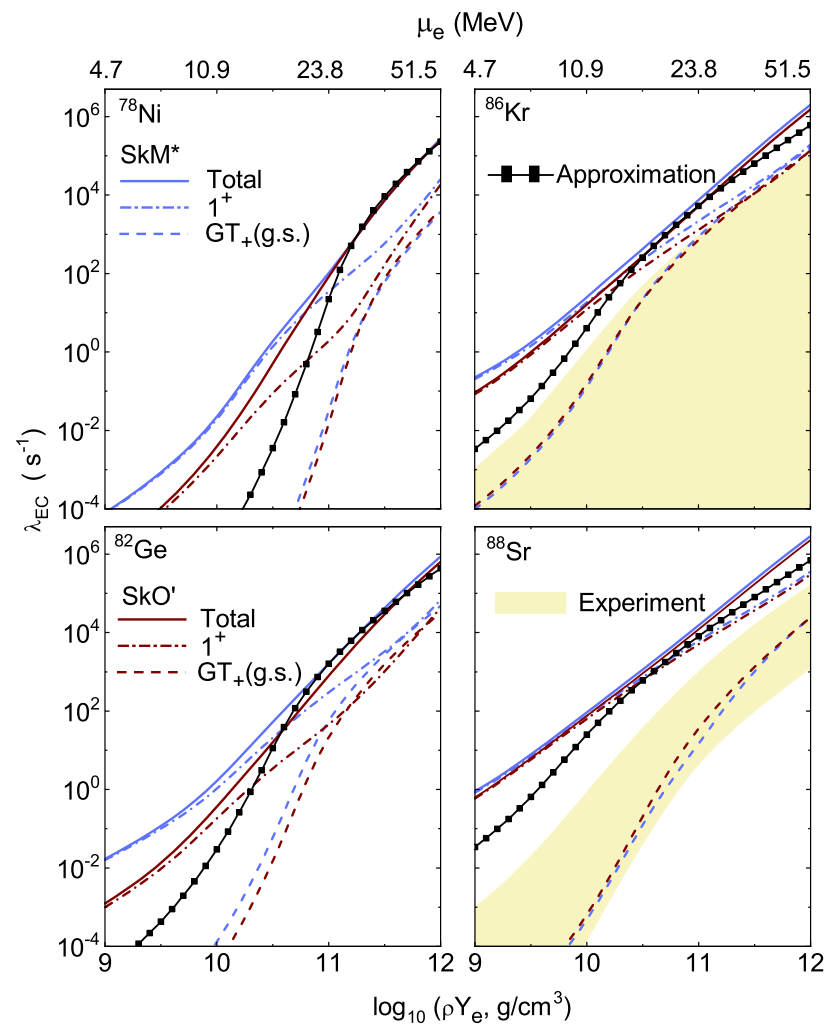

Fig. 72 EC rates calculated at $T=10 \mathrm{GK}(0.86 \mathrm{MeV})$ as a function of density. The upper axis indicates the corresponding electron chemical potentials. The blue (light gray) and brown (dark gray) lines are based on the Skyrme-thermal-QRPA calculations with the SkM* and SkO' interactions, respectively. The total rates (full lines) include the contribution of allowed $\left(0^{+}, 1^{+}\right)$and first-forbidden $\left(0^{-}, 1^{-}, 2^{-}\right)$transitions. The dashed-dotted lines (labeled $1^{+}$) represent the unblocked GT + contributions to the rates. The rates, indicated by dashed lines, have been calculated from the ground-state $\mathrm{GT}_{+}$distribution. The shaded bands represent the results based on the experimental $\mathrm{GT}_{+}$data [392,393]

where $\beta_{\lambda \mu}$ are deformation parameters. Besides $\beta_{20}$ corresponding to the elongation of the whole nucleus along its symmetry axis, other shape degrees of freedom also play a crucial role during the fission process [394-412]. Therefore, examining the multidimensional potential energy surfaces (PES) for a nucleus in question is important for obtaining detailed information about the fission properties. The EDF models have been extensively used to study fission barriers of heavy and superheavy nuclei; the reader is referred to Refs. $[88,413]$ for two recent reviews and to Refs. [410-412,414430] for more recent progress.

The multidimensionally-constrained (MDC) CEDF models have been developed to calculate and study PES for SHN and heavy nuclei $[138,405,407,431]$. The MDC-CEDF models incorporate the shape degrees of freedom $\beta_{\lambda \mu}$ with $\mu$ being even numbers, e.g., $\beta_{20}, \beta_{22}, \beta_{30}, \beta_{32}, \beta_{40}, \beta_{42}$ and $\beta_{44}$. The MDC-CEDF models have been applied to the study of the fission barriers and PES of actinide nuclei [405,407], the third minima in PES of light actinides [432], the non- axial octupole $Y_{32}$ correlations in $N=150$ isotones [433] and $\mathrm{Zr}$ isotopes [431], axial octupole correlations in multiple chiral doublet [434] and Ba isotopes [435] and the shapes of hypernuclei $[436,437]$. Based on the PES from MDC-CEDF models, the dynamics of spontaneous and induced fissions in actinide nuclei has been studied [425,427,438,439].

Pairing correlations and the way to deal with them are crucial in determining the barrier height for nuclear fission [440]. Either the BCS approach or the Bogoliubov transformation is implemented in MDC-CEDF models for considering pairing correlations. The MDC-CEDF model with the BCS approach used for treating pairing correlations is named the MDCRMF model, whereas that with the Bogoliubov transformation is called the MDC relativistic Hartree-Bogoliubov model. In the study of fission barriers and PES of actinide nuclei with the MDC-RMF model, the reflection-asymmetric octupole shape and triaxial deformation were revealed to be crucial for the second fission barriers in actinide nuclei $[405,407]$. Thus, the question arises of what role the triaxial and octupole deformations play in SHN. The systematic study of the fission barriers for even-even SHN with $Z=112-120$ was performed in Ref. [402] by using the RMF model with triaxial and octupole shapes included separately. It is found that the triaxiality has a considerable impact on the shape and the height of outer fission barrier, which is lowered by $\sim 3 \mathrm{MeV}$ in the ${ }^{292} 120$ nucleus. Interestingly, it was also seen that, in this nucleus, the lowering of the outer fission barrier due to the octupole deformation is substantially smaller than the lowering effect due to the triaxiality. Therefore in the RMF calculations, the lowering of the triaxial deformation on the second fission barrier exists not only in actinide nuclei but also in SHN.

Among SHN, ${ }^{270} \mathrm{Hs}$ is of particular interest because it is well deformed and exhibits the deformed doubly magic feature. This nucleus has been investigated within the EDF models with the axial and/or reflection-symmetric shapes assumed [441-448]. Recently the MDC-RMF model has been used to make a thorough study of the ground-state properties, PES and fission barriers in ${ }^{270} \mathrm{Hs}$ [412]. The first PES has been presented for ${ }^{270} \mathrm{Hs}$ with both triaxiality and reflection asymmetry considered. Thus, one can examine in detail the influences of the axial octupole deformation $\beta_{30}$ and nonaxial quadrupole deformation $\beta_{22}$ on the PES and fission barriers.

In the MDC-RMF model, both the triaxial (TA) and the reflection-asymmetric (RA) deformations are allowed. We may switch off TA or RA deformations and keep the nucleus in question axially symmetric (AS) or reflection symmetric (RS). Thus, four typical combinations of symmetries can be imposed in the MDC-RMF calculations: AS-RS, AS-RA, TA-RS and TA-RA [412].

Four potential energy curves (PECs) of ${ }^{270} \mathrm{Hs}$ calculated from the MDC-RMF model are shown in Fig. 73. These four 


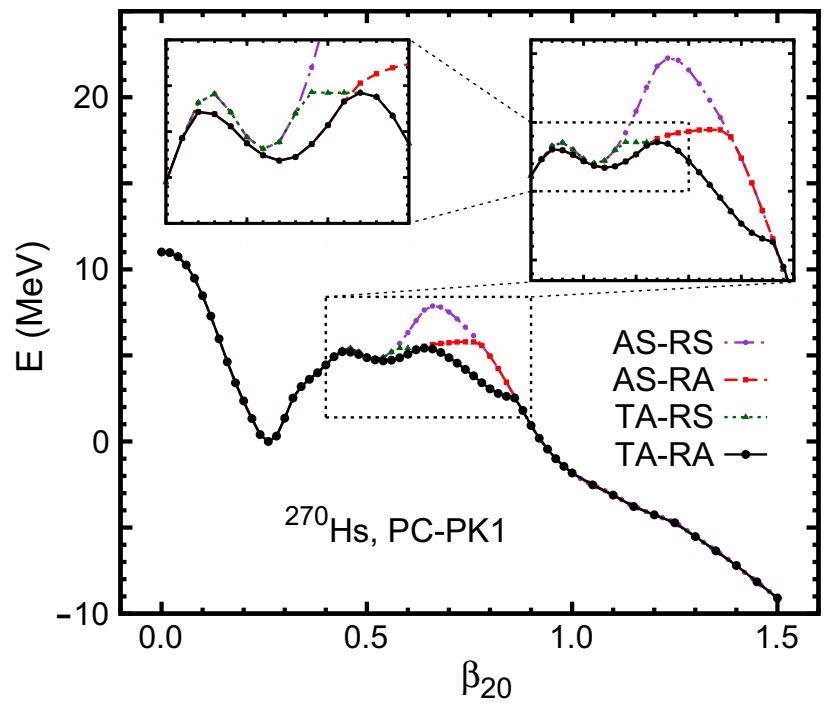

Fig. 73 PEC of ${ }^{270} \mathrm{Hs}$ obtained from MDC-RMF calculations with four combinations of symmetries imposed: axially symmetric and reflection-symmetric (AS-RS) deformations, axially symmetric and reflection-asymmetric (AS-RA) deformations, triaxial and reflection-symmetric (TA-RS) deformations, and triaxial and reflectionasymmetric (TA-RA) deformations. The inset on the top right shows these four PECs in the region $0.4 \leq \beta_{20} \leq 0.9$ beyond which the four curves are identical. The inset on the top left shows these PECs in the region $0.4 \leq \beta_{20} \leq 0.7$. Note that the TA-RS PEC overlaps with the TA-RA curve when $\beta_{20} \geq 0.64$ and the AS-RA and TA-RA PECs overlap with each other when $\beta_{20} \leq 0.62$. Taken from Ref. [412]

PECs are identical in the following $\beta_{20}$ intervals: $0 \leq \beta_{20} \leq$ 0.42 and $0.88 \leq \beta_{20} \leq 1.50$. In particular, the global minimum corresponding to the ground state of ${ }^{270} \mathrm{Hs}$ coincides in all these four calculations regardless of the imposed symmetries. For the AS-RS PEC in Fig. 73, the first fission barrier occurs at around $\beta_{20}=0.46$ and the height of this barrier is $5.4 \mathrm{MeV}$. The second minimum in the AS-RS PEC occurs at $\beta_{20}=0.52$ and the energy of this minimum is $4.8 \mathrm{MeV}$ above the ground state. The depth of the pocket around the second minimum is about $0.6 \mathrm{MeV}$. The second fission barrier is around $\beta_{20}=0.66$ with a height of $7.9 \mathrm{MeV}$. If the RA is considered, one gets the AS-RA PEC in which the height of the first barrier is $5.2 \mathrm{MeV}$, which is by $0.2 \mathrm{MeV}$ lower than that in the AS-RS curve. The second minimum is shifted to $\beta_{20}=0.54$ and the energy is by $0.1 \mathrm{MeV}$ smaller than that from the RA calculation. Though it does not affect the first barrier and the second minimum very much, the reflectionasymmetric shape has a considerably large effect on the second fission barrier which is not only shifted to $\beta_{20}=0.74$ but also lowered by $2.1 \mathrm{MeV}$ (cf. violet and red curves in Fig. 73). The TA-RS PEC still has a double-hump structure and the depth of the pocket around the second minimum is about $0.6 \mathrm{MeV}$. As seen, the TA distortion does not influence much the first barrier and the second minimum, but lowers the second fission barrier by about $2.4 \mathrm{MeV}$. This lowering effect is very pronounced considering that the height of the second barrier in the AS-RS PEC is only 7.9 MeV. The fact that the triaxiality affects only the second fission barrier in ${ }^{270} \mathrm{Hs}$ is different from the findings in actinides in which the triaxial deformations lower both the first and the second fission barriers considerably $[405,407]$. When both triaxial and reflection-asymmetric shapes are allowed in the MDC-RMF calculations, we obtain the TA-RA PEC which is shown in Fig. 73 by black dots connected by a solid line. Compared with the TA-RS PEC, the TA-RA curve is slightly lower in the region $0.44 \leq \beta_{20} \leq 0.62$ as can be seen more clearly in the insets of Fig. 73. We note that the RS PECs have been obtained for ${ }^{270} \mathrm{Hs}$ from Woods-Saxon-Strutinsky calculations in reflection-symmetric deformation space $\left(\beta_{2}, \gamma, \beta_{4}\right)$ [417].

In Fig. 73, the energy is continuous as a function of $\beta_{20}$ along the four PECs. However, the $\beta_{30} \sim \beta_{20}$ and $\beta_{22} \sim \beta_{20}$ curves are discontinuous along the AS-RA, TA-RS and TARA PECs. These sudden changes of $\beta_{30}$ or $\beta_{22}$ in the ASRA, TA-RS and TA-RA PECs come from the complexity of multidimensional PES. In the self-consistent calculations constraining only $\beta_{20}$, shape degrees of freedom other than $\beta_{20}$ are minimized automatically in the process of finding (local) minima in a PES. It often happens that there are two or more valleys in a higher-dimensional PES. When such a PES is projected onto a lower-dimensional one, these valleys may overlap or be connected abruptly, resulting in "continuous" PES (e.g., the AS-RA, TA-RS and TA-RA PECs in Fig. 73) though the deformation parameters which are projected out may change suddenly at intersecting or connecting point(s). It is clear that one needs to examine higher-dimensional PES in order to eliminate such discontinuities.

2D constraint RMF calculations are carried out for ${ }^{270} \mathrm{Hs}$ with both triaxial and reflection-asymmetric shapes allowed. The 2D PES obtained is shown in Fig. 74. There are two competing fission paths, the RS path along the $\beta_{20}$-axis with $\beta_{30}=0$ and the RA path going to the top right corner of this plot. In this PES, there are some discontinuities, e.g., around $\left(\beta_{20} \approx 1.2, \beta_{30} \approx 0.7\right),\left(\beta_{20} \approx 1.5, \beta_{30} \approx 1.4\right)$ and $\left(\beta_{20} \approx 1.9, \beta_{30} \approx 1.8\right)$. These discontinuities may also be due to the complexity of multidimensional PES. To resolve these discontinuities, one has to investigate 3D PES by making 3D constraint calculations on $\beta_{20}, \beta_{30}$ and $\beta_{22}$.

By examining $\beta_{22}$ values of the points in the PES shown in Fig. 74, it is found that the TA deformation is zero along the RA fission path. However, the TA most effects are significant along the RS fission path. In Fig. 75, the PECs of ${ }^{270} \mathrm{Hs}$ corresponding to the RS and RA fission paths in Fig. 74 are presented. In contrast with the RA path, the RS fission path is considerably lowered by the TA distortion. As a result, the fission barrier along the RS path is much lower than that in the RA path. Comparing Figs. 73 and 75, we finally come to the conclusion that the TA-RS PEC corresponds to the lowest 


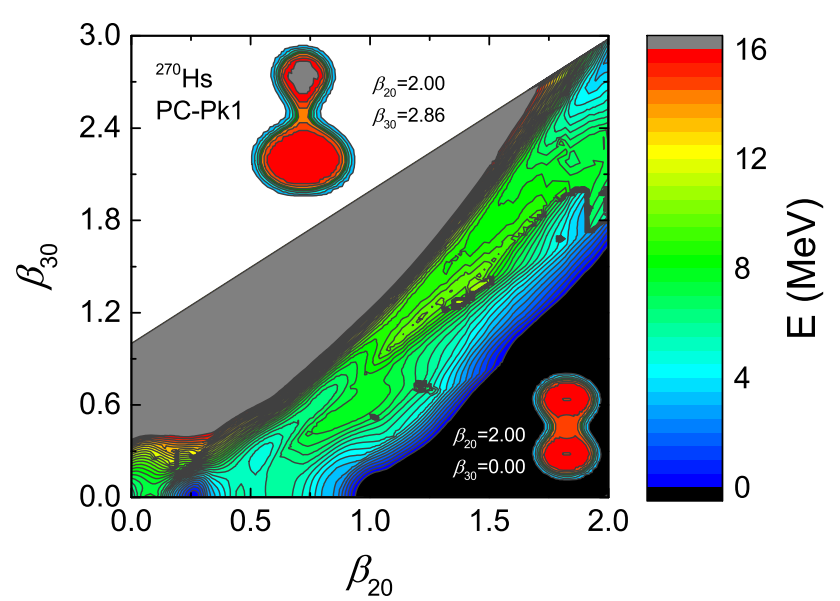

Fig. 74 PES of ${ }^{270} \mathrm{Hs}$ obtained from $2 \mathrm{D}$ constraint RMF calculations with triaxial and reflection-asymmetric (TA-RA) deformations allowed. The energy is normalized with respect to the binding energy of the ground state. The contour interval is $0.5 \mathrm{MeV}$. The density profiles of ${ }^{270} \mathrm{Hs}$ at $\left(\beta_{20}=2.00, \beta_{30}=2.86\right)$ and $\left(\beta_{20}=2.00, \beta_{30}=0\right)$ are shown. Taken from Ref. [412]

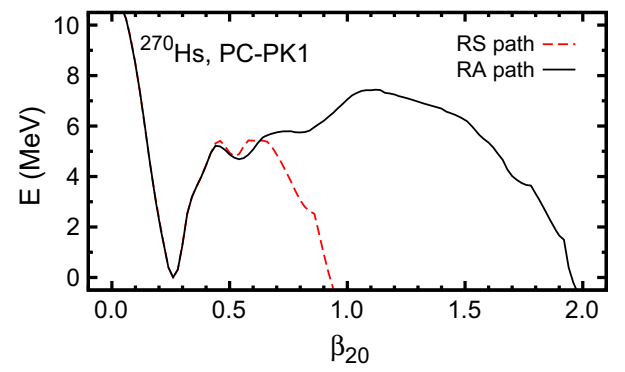

Fig. $75 \mathrm{PEC}$ of ${ }^{270} \mathrm{Hs}$ corresponding to the reflection-symmetric (RS) and reflection-asymmetric (RA) fission paths in Fig. 74 but recalculated with the ADHO basis truncated with $N_{f}=20$. The energy is normalized with respect to the binding energy of the ground state. Note that the two curves overlap with each other when $\beta_{20}<0.44$. Taken from Ref. [412]

static fission path, though it is higher in energy than the TARA cur in Fig. 73. Such a conclusion can only be reached when both 1D PEC and 2D PES are examined carefully and when both triaxial and reflection-asymmetric deformations are considered.

\subsection{Spontaneous fission modes and lifetimes}

One of the key issues in nuclear physics is the prediction of the half-lives of actinides and SHN and the competition between $\alpha$-decay and spontaneous fission. Because the $\alpha$-decay chains of the SHN mostly terminate by spontaneous fission of unknown nuclei, the study of the spontaneous fission is necessary for the unambiguous identification of the SHN. The half-life calculations over a wide range of SHN are performed in Refs. [397,402,449-452], using non-relativistic and relativistic self-consistent mod- els. A systematic study of these nuclei is performed in the HFB approach with the finite-range and density-dependent Gogny force in the D1S parametrization [406]. The evolution of the spontaneous fission half-life is carried out in the standard WKB framework, where the fission barrier tunneling occurs along the collective quadrupole moment $Q_{2}$ constrained path. For the collective quadrupole inertia, the adiabatic time-dependent HFB expression is used and computed in the cranking approximation. Three different fission paths for each nucleus corresponding to the constraints on the wave functions are considered in Fig. 76: 1) the axially symmetric $(\gamma=0)$ and reflection-symmetric $\left(Q_{3}=0\right)$ path (ASRS), 2) the axially symmetric and non-reflection-symmetric $\left(Q_{3} \neq 0\right)$ path (AS-NRS), and 3) the non-axially-symmetric $(\gamma \neq 0)$ and reflection-symmetric path (NAS-RS). For the comparison, the $\alpha$-decay half-lives are also calculated using the phenomenological formula of Viola and Seaborg and the calculated values of $Q_{\alpha}$. The half-lives for $\alpha$-decay and spontaneous fission for even-even nuclei with $Z=100-126$ are presented in Fig. 76 where the isotopic chain of each even$Z$ element is shown in a separate panel. The $\alpha$-emission is the predominant decay mode, especially in the proton-rich region. The reflection-symmetric fission mode is dominant for the proton-deficient SHN with $Z \leq 104$ and $N \geq 158$, $Z \leq 108$ and $N \geq 170, Z \leq 116$ and $N \geq 186$. The fastest decay in the non-reflection-symmetric fission channel occurs for SHN with $108 \leq Z \leq 114$ and $170 \leq N \leq 180$. The regions of anomalous long-living nuclei, for example, in the vicinities of ${ }^{268} \mathrm{Sg}$, neutron-rich Hs isotopes, and protondeficient isotones with $N=178,182,184$, are predicted. The nuclei beyond $Z=120$ and $N=184$ have short half-lives. In general, these calculations reproduce the global trend of the measured half-lives and the enhanced stability against fission of nuclei with $N=152$ and 162 neutron closed shells. Since the model [406] overestimates the absolute values of the halflives, fine-tuning of the EDF used is necessary. Many authors $[93,94,315,454-460]$ have discussed the crucial role that an accurate adjustment of the surface energy coefficient of an EDF's parametrization plays for large-amplitude collectivemotion phenomena like fission of nuclei. These are examples of the attempts to constrain the surface properties of effective interactions by looking for a relationship between microscopic and macroscopic models. For this purpose, one can also use a link between the self-consistent microscopic and Mic-Mac [415,419,420,461,462] models.

\subsection{Cluster radioactivity}

Cluster radioactivity is an exotic nuclear decay mode observed in actinides (from ${ }^{221} \mathrm{Fr}$ to ${ }^{242} \mathrm{Cm}$ ) where a light nucleus $\left({ }^{14} \mathrm{C},{ }^{20} \mathrm{O},{ }^{23} \mathrm{~F},{ }^{24-26} \mathrm{Ne},{ }^{28-30} \mathrm{Mg},{ }^{32,34} \mathrm{Si}\right)$ is emitted. Because the heavy-mass residue is always the doubly magic ${ }^{208} \mathrm{~Pb}$ or a nucleus in its neighborhood, this process is 
Fig. 76 The spontaneous fission and $\alpha$-emission half-lives in heavy actinides and SHN. Experimental data are taken from Refs. [1,3,453]. Taken from Ref. [406]

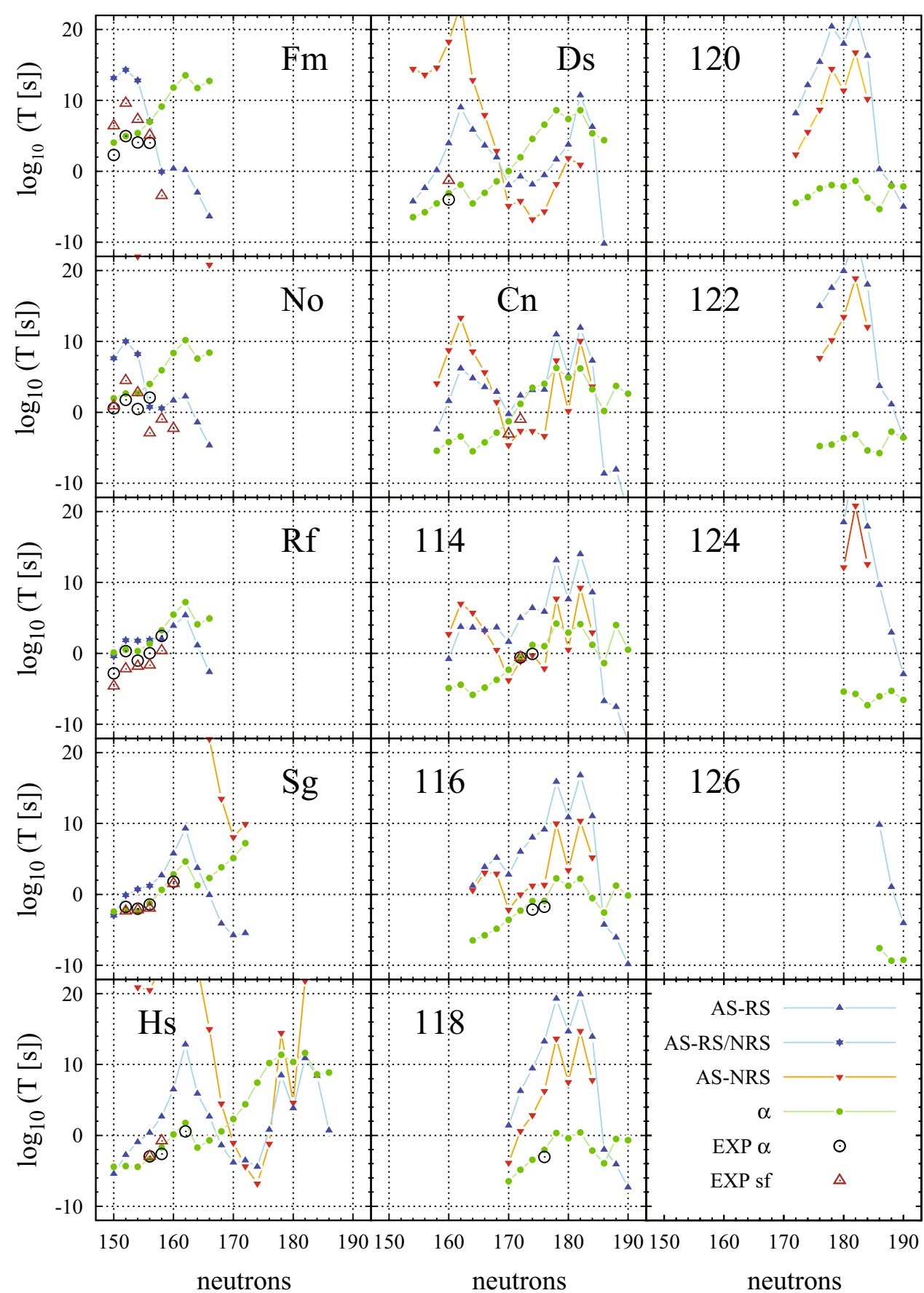

also called "lead radioactivity". The half-life of this exotic process is in the range from $10^{15} \mathrm{~s}$ to $10^{25} \mathrm{~s}$. As shown in Ref. [463], the cluster radioactivity is a kind of very asymmetric spontaneous fission. A new fission valley leading to a decay with large fragment mass asymmetry matching the cluster radioactivity products is found. The dynamics of very asymmetric spontaneous fission is governed by the shape of the potential energy surface given as a function of the axially symmetric octupole and quadrupole moments and computed in a fully microscopic mean-field HFB theory with the phenomenological Gogny D1S interaction [463]. The octupole moment is found to be more convenient than the standard quadrupole one as the parameter driving the system to fission. A continuous fission path through the scission point is described using the neck parameter constraint. The calculated cluster radioactivity half-lives of even-even actinide nuclei with $Z \leq 96$ are in noticeable agreement with the existing experimental data. Within the same self-consistent microscopic model, the standard cluster radioactivity observed in the light actinides is studied in heavier nuclei up to ${ }^{294} \mathrm{Lv}$ ( $Z=116$ ) [464]. It was found that this super-asymmetric fission mode with doubly magic ${ }^{208} \mathrm{~Pb}$ as the heavy fragment 
becomes the dominant decay mode of SHN with $Z \geq 112$. As a result, the maximum of the mass distribution of fission fragments is predicted to be around $A=208$ [464]. Note that the same conclusion about the mass distribution of SHN is given within the Mic-Mac approaches [465,466]. So, if standard cluster radioactivity is directly connected with a very asymmetric spontaneous fission, then it can be experimentally proved, for example, measuring the spontaneous fission of ${ }^{284} \mathrm{Cn}$. It is clear that the possible fragment mass asymmetry forced by the shell structure of ${ }^{208} \mathrm{~Pb}$ should also exist in the case of induced fission, if it exists at spontaneous fission.

\section{Summary}

The merits of combining macroscopic and microscopic approaches for the exploration of the vastly unknown territory of SHN has been the motif of this review article. The methods of EDF theory have been discussed as a powerful tool for large scale theoretical investigations over almost the whole nuclear mass table from light to SHN. The derivation of nuclear EDF by first principles, guided by and based on microscopic nuclear many-body theory, was presented. Single-particle self-energies, the pairing field, and residual interactions were determined by variational principles, ensuring self-consistency at all levels, from single-particle motion and nuclear ground-state properties to nuclear excitations. The Giessen EDF was discussed in detail, exemplifying the microscopic derivation of nuclear EDF. Observables of infinite nuclear matter and ground properties of finite nuclei were discussed. An inversion method was introduced, allowing one to derive the effective EDF compatible with phenomenological mean-field potentials. Broad space was given to establishing the connection to the phenomenological Mic-Mac method and putting it on firmer foundations by improving the microscopic content. The reduction of the non-relativistic and the relativistic mean-field wave equations to a form compatible with the Mic-Mac formalism was presented in detail, resulting in the definition of Schrödinger-equivalent potentials. Phenomenological EDF methods were illustrated by the FEDF. The FEDF approach incorporates as limiting case the class of the widely used SkEDFs, but in its general form includes a much richer operator structure in the particle-hole and the particle-particle sectors.

Because of practical numerical reasons the selfconsistently derived Schrödinger-equivalent potentials were parameterized by the WS form factors. The mass-charge dependence of the form-factor parameters was derived in analytical form. Accordingly, the self-consistent HFB proton and neutron ground-state density for spherical and deformed nuclei were parameterized in terms of Fermi distributions. The goal of these exercises was to obtain insight in the iso- topic and isotonic dependencies of radius and diffuseness parameters with the virtue of extrapolations and applications in the SHN mass region. The isotopic dependence of the nuclear radius parameter is found to be weak, while the value of the nuclear diffuseness depends quite strongly on the neutron number. If the neutron shell is closed by a level with large orbital angular momentum, $a(N)$ approaches a minimum at that magic neutron number $N$. In other words, the centrifugal barrier of high-angular momentum orbits induces a suppression of the diffuseness of the nuclear surface. The investigations imply an intuitive simple dependence of the diffuseness on the separation energy, $a(N) \sim 1 / \sqrt{S_{n}}$.

As shown, the value of the diffuseness is related to the attraction of the $N N$ interaction. The strength of the density-dependent $N N$ forces at low densities strongly influences the height of the Coulomb barrier in the nucleusnucleus interaction potential, reflected clearly in observables. The nucleus-nucleus interaction potential is defined by the density-dependent $N N$ interaction and nucleon-density profiles. Calculation with larger diffuseness $a$ and weaker asymptotic attraction could result in the same barrier height as the calculation with smaller $a$ and stronger asymptotic attractions. These observations may serve as an additional constraint on the low density properties of the EDF, which otherwise are hardly assessed by binding energy calculations alone. Thus, the description of the experimental heights of the Coulomb barriers can be used to adjust the EDF parameters. This can be employed to fine-tune the EDF.

The polarization of the nuclear surface for neutrons slightly differs from the one for protons and the pairing correlations have a strong influence on the surface deformation and skin polarization. The polarization of the nuclear surface can directly affect some aspects of the nuclear dynamics. Among them, one can anticipate specific features in collective motion built on deformed nuclei and possible modification of the fusion, fission, quasifission, $\alpha$-decay, and cluster-emission barriers.

The mean-field potentials extracted from the GEDF/HFB calculations were applied in the Mic-Mac method for calculating the ground-state shell corrections, the mass excesses, and $Q_{\alpha}$-values in the superheavy nuclei with $Z=112$ 120. We found that the self-consistent approach provides deeper single-particle potentials that those used in the phenomenological consideration and endorses the stronger shell effects at $Z=120$ rather than those at $Z=114$. The reason for these potential differences is mainly related to the inclusion of momentum-dependent effects. In the selfconsistent potentials the momentum dependence, i.e. nonlocality, of the underlying microscopic mean-field selfenergies is accounted for by taking an average over the Fermi sphere. So, the Mic-Mac treatment qualitatively leads to results close to those in the self-consistent mean-field treatments. 
According to the predictions of the Mic-Mac method with the mean-field potential extracted from the GEDF model, one can expect the production of superheavy evaporation residues with $Z=119$ and 120 in the reactions ${ }^{50} \mathrm{Ti}+{ }^{249} \mathrm{Bk}$ and ${ }^{50} \mathrm{Ti}+{ }^{249} \mathrm{Cf}$ with cross-sections of 19 and $8 \mathrm{fb}$, respectively. The $Z=120$ nuclei with $N=175-179$ are predicted to have $Q_{\alpha}$ about $12.0-11.2 \mathrm{MeV}$ and lifetimes about $3 \mathrm{~ms}-$ 0.2 s. These $Q_{\alpha}$ are in fair agreement with Ref. [266-269] and about $2 \mathrm{MeV}$ smaller than in Refs. [230,263,264]. The experimental measurement of $Q_{\alpha}$ for at least one isotope of the $Z=120$ nucleus would help tremendously to check the theoretical calculations and, if necessary, to adjust parameters to the proper shell structure of the SHN with $Z>118$. Note that the definition of the maxima of the excitation functions provides a good test for the predictions of the models as well.

The spectra of low-lying non-rotational states in the nuclei of $\alpha$-decay chains of ${ }^{295} 119$ and ${ }^{295,297} 120$ were studied. Based on the calculated one-quasiproton spectra and energies for $\alpha$-decays, we could explain why the $\alpha$-decay chain of ${ }^{295} 119$ or ${ }^{291} \mathrm{Ts}$, or ${ }^{287} \mathrm{Mc}$ is terminated by spontaneous fission of ${ }^{267} \mathrm{Db}$. In addition, the number of isomeric states in the heaviest odd- $Z$ nuclei were predicted. In ${ }^{295} 119$ nucleus, for example, we expect the low-lying isomeric state $13 / 2^{+}$[606]. The $\alpha$-decays from some of the isomeric states seem to be possible, resulting in a possible spread of the alpha energies across an energy window of almost $0.7 \mathrm{MeV}$. The $\alpha$-decay $Q_{\alpha}$-values of nuclei belonging to the $\alpha$-decay chain of ${ }^{295} 119$ and ${ }^{295,297} 120$ were obtained and compared with the available experimental data. A rather good description of the data was demonstrated.

As shown within non-relativistic EDF models, there is in general no room for $\beta^{+} / \mathrm{EC}$-decay to play a role in the decays of SHN produced in the hot-fusion reactions [374]. The $\alpha$ decay half-lives are many orders of magnitude smaller than the $\beta^{+} / \mathrm{EC}$-decays half-lives and, therefore, there is almost no possibility of the production of new more neutron-rich SHN with $Z>112$ in the $\beta^{+} /$EC-decay channel. It should also be noted that there is currently a great interest in such processes as $\beta$-decay and $\beta$-delayed multi-neutron emission in heavy nuclei. The self-consistent methods [379,380,390,391] show success in describing these processes. Further improvement of these methods can be carried out by improving the isospin dependence in the EDF.

As shown, the self-consistent methods provide a firm basis to study the properties of SHN including their quasiparticle structure, $\beta$-decay, EC, and fission paths. However, the results of these methods have some uncertainties because of the phenomenological definition of the EDF. Besides considerable progress in the description of the nuclear structure with the self-consistent methods, there are a lot of unsolved problems related to nuclear large-amplitude dynamics responsible for cluster radioactivity, fission, and quasifission processes.
By looking for a relationship between microscopic and MicMac models, one can constrain the parameters of the effective interactions. The development of new approaches is required to construct the EDF and reach the accuracy in the prediction of the nuclear properties like in the Mic-Mac models. In this respect, the proposed deconvolution method may be useful, which allows us to reconstruct the proper EDF by inversion from the experimental data.

Combining the self-consistent approach with the MicMac method, one could find help using the advantage of each method to simplify the calculations for strongly deformed nuclear states and fission barriers. This combination is also important for the cross check of the two methods and comparison of the results with the experimental data available. As shown, the WS single-particle potential extracted from the self-consistent approaches results in a good description of the single-particle spectra, fully competitive with the phenomenological WS models. An interesting future project is to incorporate more details of the single-particle potential, e.g. the bottom oscillations induced by shell effects, into the MicMac approaches and study their influence on the SHN properties. The study of a link between the non-relativistic and relativistic EDF models may allow us to deepen our understanding of the interplay between single-particle central and spin-orbital potentials for the Mic-Mac approach.

The work of G.G.A. and N.V.A. was supported by Ministry of Science and Higher Education of the Russian Federation (contract 075-10-2020-117). The supports of RFBR (20-02-00176) and DFG (grant Le439/16) are acknowledged. S.G.Z. was partly supported by the National Key R\&D Program of China (2018YFA0404402), the NSF of China (11525524, 12070131001, 12047503, and 11961141004), the CAS Key Research Program of Frontier Sciences (QYZDB-SSWSYS013), the CAS Key Research Program (XDPB09-02), the Inter-Governmental S\&T Cooperation Project between China and Croatia, the IAEA CRP 'F41033", the HPC Cluster of KLTP/ITP-CAS and the Supercomputing Center, CNIC of CAS.

Data Availability Statement This manuscript has no associated data or the data will not be deposited. [Authors' comment: Any data that support the findings of this study are included within the article.]

\section{References}

1. Y.T. Oganessian, J. Phys. G 34, R165 (2007)

2. Y.T. Oganessian et al., Phys. Rev. C 69, 054607 (2004)

3. Y.T. Oganessian et al., Phys. Rev. C 72, 034611 (2005)

4. Y.T. Oganessian et al., Phys. Rev. C 76, 011601 (2007)

5. Y.T. Oganessian et al., Phys. Rev. Lett. 104, 142502 (2010)

6. Y.T. Oganessian et al., Phys. Rev. C 87, 014302 (2013)

7. Y.T. Oganessian et al., Phys. Rev. C 87, 034605 (2013)

8. Y.T. Oganessian et al., Phys. Rev. C 87, 054621 (2013)

9. Y.T. Oganessian, V.K. Utyonkov, Nucl. Phys. A 944, 62 (2015) 
10. V.K. Utyonkov et al., Phys. Rev. C 92, 034609 (2015)

11. S. Hofmann et al., Eur. Phys. J. A 32, 251 (2007)

12. S. Hofmann, Radiochim. Acta 99, 405 (2011)

13. S. Hofmann et al., Eur. Phys. J. A 48, 62 (2012)

14. S. Hofmann, J. Phys. G 42, 114001 (2015)

15. S. Hofmann et al., Eur. Phys. J. A 52, 180 (2016)

16. R. Eichler et al., Nature 447, 72 (2007)

17. L. Stavsetra, K.E. Gregorich, J. Dvorak, P.A. Ellison, I. Dragojević, M.A. Garcia, H. Nitsche, Phys. Rev. Lett. 103, 132502 (2009)

18. Ch. Düllmann et al., Phys. Rev. Lett. 104, 252701 (2010)

19. J.M. Gates et al., Phys. Rev. C 83, 054618 (2011)

20. D. Rudolph et al., Phys. Rev. Lett. 111, 112502 (2013)

21. J.M. Khuyagbaatar et al., Phys. Rev. Lett. 112, 172501 (2014)

22. S. Hofmann, G. Münzenberg, Rev. Mod. Phys. 72, 733 (2000)

23. K. Morita et al., J. Phys. Soc. Jpn 73, 2593 (2004)

24. K. Morita et al., Eur. Phys. J. A 21, 257 (2004)

25. K. Morita et al., J. Phys. Soc. Jpn. 76, 043201 (2007)

26. D. Lunney, J.M. Pearson, C. Thibault, Rev. Mod. Phys. 75, 1021 (2003)

27. C.F. Weizsäcker, Z. für Physik 96, 431 (1935)

28. H.A. Bethe, R.F. Bacher, Rev. Mod. Phys. 8, 82 (1936)

29. V.M. Strutinsky, Sov. J. Nucl. Phys. 3, 449 (1966)

30. V.M. Strutinsky, Nucl. Phys. A 95, 420 (1967)

31. S.G. Nilsson, C.F. Tsang, A. Sobiczewski, Z. Szymanski, S. Wycech, C. Gustafson, I.-L. Lamm, P. Möller, B. Nilsson, Nucl. Phys. A 131, 1 (1969)

32. A. Sobiczewski, F.A. Gareev, B.N. Kalinkin, Phys. Lett. B 2, 500 (1966)

33. H. Meldner, Ark. Fys. 36, 593 (1967)

34. S.G. Nilsson, J.R. Nix, A. Sobiczewski, Z. Szymanski, S. Wycech, C. Gustafson, P. Möller, Nucl. Phys. A 115, 545 (1968)

35. E.O. Fiset, J.R. Nix, Nucl. Phys. A 193, 647 (1972)

36. J. Randrup, S.E. Larsson, P. Möller, S.G. Nilsson, K. Pomorski, A. Sobiczewski, Phys. Rev. C 13, 229 (1976)

37. V.A. Chepurnov, Yad. Fiz. 6, 955 (1967)

38. A. Bohr, B. Mottelson, Nuclear Structure, Vol. 1 and 2 (Benjamin, NY, 1975)

39. V.G. Soloviev, Theory of complex nuclei (Pergamon Press, Oxford, 1976)

40. V.G. Soloviev, Theory of atomic nuclei: quasipartcle and phonons (Institute of Physics, Bristol, 1992)

41. V.G. Soloviev, A.V. Sushkov, N.Y. Shirikova, Phys. Part. Nucl. 25, 157 (1994)

42. S.P. Ivanova, A.L. Komov, L.A. Malov, V.G. Soloviev, Phys. Part. Nucl. 7, 450 (1976)

43. V.G. Soloviev, A.V. Sushkov, N.Y. Shirikova, Phys. Part. Nucl. 27, 667 (1996)

44. J. Maruhn, W. Greiner, Z. Phys. A 251, 431 (1972)

45. P. Möller, J.R. Nix, W.D. Myers, W.J. Swiatecki, At. Data Nucl. Data Tables 59, 185 (1995)colon http://t2.lanl.gov/nis/data/astro/ molnix 96/molnix.html

46. J. Dudek, Z. Szymanski, T. Werner, A. Fässler, C. Lima, Phys. Rev. C 26, 1712 (1982)

47. I. Ahmad, Phys. Scr. T. 125, 78 (2006)

48. I. Ahmad, R.R. Chasman, Phys. Rev. C 80, 064315 (2009)

49. N. Schwierz, I. Wiedenhoever, A. Volya, arXiv:nucl-th/07093525

50. N. Wang, M. Liu, X. Wu, J. Meng, Phys. Lett. B 734, 215 (2014); http://www.imqmd.com/mass/

51. P. Möller, J.R. Nix, J. Phys. G 20, 1681 (1994)

52. A. Sobiczewski, Phys. Part. Nuclei 25, 295 (1994)

53. R. Smolanczuk, J. Skalski, A. Sobiczewski, Phys. Rev. C 52, 1871 (1995)

54. I. Muntian, Z. Patyk, A. Sobiczewski, Acta Phys. Pol. B 32, 691 (2001)
55. I. Muntian, Z. Patyk, A. Sobiczewski, Acta. Phys. Pol. 34, 2141 (2003)

56. I. Muntian, S. Hofmann, Z. Patyk, A. Sobiczewski, Acta Phys. Pol. B 34, 2073 (2003)

57. I. Muntian, S. Hofmann, Z. Patyk, A. Sobiczewski, Phys. At. Nucl. 66, 1015 (2003)

58. A. Parkhomenko, I. Muntian, Z. Patyk, A. Sobiczewski, Acta Phys. Pol. B 34, 2153 (2003)

59. A. Parkhomenko, A. Sobiczewski, Acta Phys. Pol. B 36, 3095 (2005)

60. M. Kowal, J. Skalski, Phys. Rev. C 82, 054303 (2010)

61. P. Jachimowicz, M. Kowal, J. Skalski, Phys. Rev. C 85, 034305 (2012)

62. M. Kowal, J. Skalski, Phys. Rev. C 85, 061302(R) (2012)

63. P. Jachimowicz, M. Kowal, J. Skalski Phys. Rev. C 89, 024304 (2014)

64. P. Jachimowicz, M. Kowal, J. Skalski Phys. Rev. C 95, 014303 (2017)

65. P. Jachimowicz, M. Kowal, J. Skalski Phys. Rev. C 101, 014311 (2020)

66. G.G. Adamian, N.V. Antonenko, W. Scheid, Phys. Rev. C 81, 024320 (2010)

67. G.G. Adamian, N.V. Antonenko, S.N. Kuklin, W. Scheid, Phys. Rev. C 82, 054304 (2010)

68. G.G. Adamian, N.V. Antonenko, S.N. Kuklin, B.N. Lu, L.A. Malov, S.G. Zhou, Phys. Rev. C 84, 024324 (2011)

69. A.N. Kuzmina, G.G. Adamian, N.V. Antonenko, W. Scheid, Phys. Rev. C 85, 014319 (2012)

70. Z.H. Zhang, Theoretical investigation of the spectroscopy of the transfermium and the super-heavy nuclei, Ph.D. thesis (Institute of Theoretical Physics, Chinese Academy of Sciences, 2012)

71. Z.H. Zhang, J.Y. Zeng, E.G. Zhao, S.G. Zhou, Phys. Rev. C 83, 011304R (2011)

72. Z.H. Zhang, X.T. He, J.Y. Zeng, E.G. Zhao, S.G. Zhou, Phys. Rev. C 85, 044324 (2012)

73. Z.H. Zhang, J. Meng, E.G. Zhao, S.G. Zhou, Phys. Rev. C 87, 054308 (2013)

74. P. Hohenberg, W. Kohn, Phys. Rev. 136, 864 (1964)

75. W. Kohn, L.J. Sham, Phys. Rev. 137, 1697 (1965)

76. W. Kohn, L.J. Sham, Phys. Rev. 140, 1133 (1965)

77. D. Vautherin, D.M. Brink, Phys. Lett. B 32, 149 (1970)

78. D. Vautherin, D.M. Brink, Phys. Rev. C 5, 626 (1972)

79. A. Fetter, J. Walecka, Quantum theory of many-particle systems (McGraw-Hill, San Francisco, 1971)

80. J. Decharge, D. Gogny, Phys. Rev. C 21, 1568 (1980)

81. E. Chabanat, J. Meyer, P. Bonche, R. Schäffer, P. Haensel, Nucl. Phys. A 627, 710 (1997)

82. J. Sadoudi, T. Duguet, J. Meyer, M. Bender, Phys. Rev. C 88, $064326(2013)$

83. J.W. Negele, Rev. Mod. Phys. 54, 913 (1982)

84. V.E. Oberacker, A.S. Umar, Phys. Rev. C 87, 034611 (2013)

85. K. Hagino, J.M. Yao, Phys. Rev. C 91, 064606 (2015)

86. A.S. Umar, V.E. Oberacker, C. Simenel, Phys. Rev. C 94, 024605 (2016)

87. A. Adel, T. Alharbi, Eur. Phys. J. A 53, 1 (2017)

88. N. Schunck, L.M. Robledo, Rept. Prog. Phys. 79, 116301 (2016)

89. Z. Shi, A.V. Afanasjev, Z.P. Li, J. Meng, Phys. Rev. C 99, 064316 (2019)

90. M. Kortelainen, J. Phys. G 42, 034021 (2015)

91. A. Rios, X. Roca Maza, J. Phys. G 42, 034005 (2015)

92. J. Erler, P.-G. Reinhard, J. Phys. G 42, 034026 (2015)

93. M. Korteleinen, J. McDonnell, W. Nazarewicz, P.-G. Reinhard, J. Sarich, N. Schunck, M.V. Stoitsov, S.M. Wild, Phys. Rev. C 85, 024304 (2012) 
94. M. Korteleinen, J. McDonnell, W. Nazarewicz, E. Olsen, P.-G. Reinhard, J. Sarich, N. Schunck, S.M. Wild, D. Davesne, J. Erler, A. Pastore. Phys. Rev. C 89, 054314 (2014)

95. P.D. Stevenson, M.C. Barton, Progr. Part. Nucl. Phys. 104, 142 (2019)

96. H. Nakada, Phys. Rev. C 68, 014316 (2003)

97. T. Otsuka, T. Matsuo, D. Abe, Phys. Rev. Lett. 97, 162501 (2006)

98. H. Sagawa, G. Colò, Prog. Part. Nucl. Phys. 76, 76 (2014)

99. G.F. Dai, L. Guo, E.G. Zhao, S.G. Zhou, Sci. China-Phys. Mech. Astron. 57, 1618 (2014)

100. G.F. Dai, L. Guo, E.G. Zhao, S.G. Zhou, Phys. Rev. C 90, 044609 (2014)

101. N. Wang, L. Guo, Phys. Lett. B 760, 236 (2016)

102. C. Yu, L. Guo, Sci. China-Phys. Mech. Astron. 60, 092011 (2014)

103. L. Guo, C. Shen, C. Yu, Z. Wu, Phys. Rev. C 98, 064609 (2018)

104. L. Guo, K. Godbey, U.S. Umar, Phys. Rev. C 98, 064607 (2018)

105. L. Guo, C. Simenel, L. Shi, C. Yu, Phys. Lett. B 782, 401 (2018)

106. Z. Wu, L. Guo, Phys. Rev. C 100, 014612 (2019)

107. K. Godbey, L. Guo, U.S. Umar, Phys. Rev. C 100, 054612 (2019)

108. X.Y. Li, Z.J. Wu, L. Guo, Sci. China-Phys. Mech. Astron. 62, $122011(2019)$

109. Z.J. Wu, L. Guo, Sci. China-Phys. Mech. Astron. 631, 242021 (2020)

110. J.M. Dong, X.L. Shang, Phys. Rev. C 101, 014305 (2020). https:// doi.org/10.1103/PhysRevC.101.014305 ([arXiv:2001.02937 [nucl-th]])

111. J.W. Negele, D. Vautherin, Phys. Rev. C 5, 1472 (1972)

112. F. Hofmann, H. Lenske, Phys. Rev. C 57, 2281 (1998)

113. N. Tsoneva, H. Lenske, Phys. Atom. Nucl. 79, 885 (2016)

114. H. Lenske, N. Tsoneva, Eur. Phys. J. A 55, 238 (2019)

115. H. Lenske, C. Fuchs, Phys. Lett. B 345, 355 (1995)

116. C. Fuchs, H. Lenske, H.H. Wolter, Phys. Rev. C 52, 3043 (1995). https://doi.org/10.1103/PhysRevC.52.3043, [arXiv:nucl-th/9507044 [nucl-th]]

117. F. Hofmann, C.M. Keil, H. Lenske, Phys. Rev. C 64, 034314 (2001). https://doi.org/10.1103/PhysRevC.64.034314, [arXiv:nucl-th/0007050 [nucl-th]]

118. H. Lenske, Lect. Notes Phys. 641, 147 (2004). https://doi.org/10. 1007/978-3-540-39911-7 5

119. F. Hofmann, C.M. Keil, H. Lenske, Phys. Rev. C 64, 025804 (2001). https://doi.org/10.1103/PhysRevC.64.025804, [arXiv:nucl-th/0008038 [nucl-th]]

120. C.M. Keil, F. Hofmann, H. Lenske, Phys. Rev. C 61, 064309 (2000). https://doi.org/10.1103/PhysRevC.61.064309, [arXiv:nucl-th/9911014 [nucl-th]]

121. H. Lenske, M. Dhar, T. Gaitanos, X. Cao, Prog. Part. Nucl. Phys. 98, 119 (2018). https://doi.org/10.1016/j.ppnp.2017.09.001

122. S. Typel, H.H. Wolter, Nucl. Phys. A 656, 331 (1999). https://doi. org/10.1016/S0375-9474(99)00310-3

123. T. Niksic, D. Vretenar, G.A. Lalazissis, P. Ring, Phys. Rev. C 69, 047301 (2004). https://doi.org/10.1103/PhysRevC.69. 047301, [arXiv:nucl-th/0403027 [nucl-th]]

124. S. Shen, H. Liang, W.H. Long, J. Meng, P. Ring, Prog. Part. Nucl. Phys. 109, 103713 (2019). https://doi.org/10.1016/j.ppnp.2019. 103713, [arXiv:1904.04977 [nucl-th]]

125. M. Bender, P.H. Heenen, P.G. Reinhard, Rev. Mod. Phys. 75, 121 (2003)

126. P. Ring, D. Vretenar, B. Podobnik, Nucl. Phys. A 598, 107 (1996)

127. P. Ring, Prog. Part. Nucl. Phys. 37, 193 (1996)

128. P. Ring, Z.-Y. Ma, N. Van Giai, D. Vretenar, A. Wandelt, L.-G. Cao, Nucl. Phys. A 694, 249 (2001)

129. D. Vretenar, A.V. Afanasjev, G.A. Lalazissis, P. Ring, Phys. Rep. 409, 101 (2005)

130. P. Ring, Int. Rev. Nucl. Phys. 10, 1 (2016)

131. J. Meng, H. Toki, S.-G. Zhou, S.-Q. Zhang, W.-H. Long, L.-S. Geng, Prog. Part. Nucl. Phys. 57, 470 (2006)
132. Z.-Y. Ma, J. Rong, B.-Q. Chen, Z.-Y. Zhu, H.-Q. Song, Phys. Lett. B 604, 170 (2004)

133. W.-H. Long, N. Van Giai, J. Meng, Phys. Lett. B 640, 150 (2006)

134. T. Niksic, D. Vretenar, P. Ring, Prog. Part. Nucl. Phys. 66, 519 (2011)

135. J. Meng, J. Peng, S.Q. Zhang, P.W. Zhao, Front. Phys. 8, 55 (2013)

136. H.Z. Liang, J. Meng, S.G. Zhou, Phys. Rep. 570, 1 (2015)

137. J. Meng, S.G. Zhou, J. Phys. G 42, 093101 (2015)

138. S.G. Zhou, Phys. Scr. 91, 063008 (2016)

139. J. Meng (ed.), Relativistic density functional for nuclear structure, international review of nuclear physics, vol. 10 (World Scientific, Singapore, 2016)

140. Z.X. Ren, S.Q. Zhang, J. Meng, Phys. Rev. C 95, 024313 (2017)

141. Z.X. Ren, S.Q. Zhang, P.W. Zhao, N. Itgaki, J.A. Maruhn, J. Meng, Sci. China - Phys. Mech. Astron. 62, 112062 (2019)

142. Z.X. Ren, P.W. Zhao, J. Meng, Phys. Lett. B 801, 135194 (2020)

143. Z.X. Ren, P.W. Zhao, J. Meng, Phys. Rev. C 102, 044603 (2020)

144. J. Boguta, A.R. Bodmer, Nucl. Phys. A 292(413), 413 (1977)

145. N. Van Giai, H. Sagawa, Phys. Lett. B 106, 379 (1981)

146. S.A. Fayans, S.V. Tolokonnikov, E.L. Trykov, D. Zawischa, Nucl. Phys. A 676, 49 (2000)

147. S.V. Tolokonnikov, E.E. Saperstein, Phys. At. Nucl. 73, 1684 (2010)

148. M. Baldo, P. Schuck, X. Viňas, Phys. Lett. B 663, 390 (2008)

149. M. Baldo, L.M. Robledo, P. Schuck, X. Viňas, Phys. Rev. C 87, 064305 (2013)

150. A. Bulgac, M.M. Forbes, S. Jin, R.N. Prerez, N. Schunck, Phys. Rev. C 97, 044313 (2018)

151. G.A. Lalazissis, D. Vretenar, P. Ring, Phys. Rev. C 57, 2294 (1998)

152. S.-G. Zhou, J. Meng, P. Ring, Phys. Rev. C 68, 034323 (2003)

153. P.W. Zhao, Z.P. Li, J.M. Yao, J. Meng, Phys. Rev. C 82, 054319 (2010)

154. P. Zhao, Z. Li, Int. J. Mod. Phys. E 27, 1830007 (2019)

155. J. Li, W. Long, J. Margueron, N. Van Giai, Phys. Lett. B 732, 169 (2014)

156. J. Kvasil, V.O. Nesterenko, A. Repko, W. Kleinig, P.-G. Reinhard, Phys. Rev. C 94, 064302 (2016)

157. V.O. Nesterenko, A. Repko, J. Kvasil, P.-G. Reinhard, Phys. Rev. Lett. 120, 182501 (2018)

158. H. Gil, P. Papakonstantinou, C.H. Hyun, Y. Oh, Phys. Rev. C 99, 064319 (2019)

159. H. Gil, Y.-M. Kim, C.H. Hyun, P. Papakonstantinou, Y. Oh, Phys. Rev. C 100, 014312 (2019)

160. M. Grasso, Prog. Part. Nucl. Phys. 106, 256 (2019)

161. J. Speth, P.-G. Reinhard, V. Tselyaev, N. Lyutorovich, Phys. Rev. C 102, 054332 (2020)

162. Y.N. Zhang, S.K. Bogner, R.J. Furnstahl, Phys. Rev. C 98, 064306 (2018)

163. C. Fuchs, H. Lenske, H.H. Wolter, Phys. Rev. C 52, 3043 (1995)

164. F. de Jong, H. Lenske, Phys. Rev. C 57, 3099 (1998)

165. M. Stoitsov, M. Kortelainen, S.K. Bogner, T. Duguet, R.J. Furnstahl, B. Gebremariam, N. Schunck, Phys. Rev. C 82, 054307 (2010)

166. R. Navarro Perez, N. Schunck, A. Dyhdalo, R.J. Furnstahl, S.K. Bogner, Phys. Rev. C 97, 054304 (2018)

167. P.-G. Reinhard, H. Flocard, Nucl. Phys. A 584, 467 (1995)

168. Z.X. Ren, P.W. Zhao, Phys. Rev. C 102, 021301(R) (2020)

169. G.G. Adamian, L.A. Malov, N.V. Antonenko, H. Lenske, K. Wang, S.G. Zhou, Eur. Phys. J. A 54, 170 (2018)

170. L.D. Landau, Sov. Phys. JETP 3, 920 (1957)

171. A. Migdal, Theory of finite fermi systems and application to atomic nuclei (Wiley, New York, 1967)

172. G. Baym, S.A. Chin, Nucl. Phys. A 262, 527 (1976)

173. J. Speth, E. Werner, W. Wild, Phys. Rep. 33, 127 (1977)

174. V.A. Khodel, E.E. Saperstein, Phys. Rep. 92, 183 (1982)

175. S. Kamerdzhiev, J. Speth, G. Tertychny, Phys. Rep. 393, 1 (2004) 
176. F. Gruemmer, J. Speth, J. Phys. G 32, R193 (2006)

177. J. Dobaczewski, J. Phys. G 43, 1 (2016)

178. J.W. Negele, D. Vautherin, Phys. Rev. C 11, 1031 (1975)

179. N. Tsoneva, S. Goriely, H. Lenske, R. Schwengner, Phys. Rev. C 91, 044318 (2015)

180. A. Akmal, V.R. Pandharipande, D.G. Ravenhall, Phys. Rev. C 58, 1804 (1998)

181. L. Gorkov, JETP 9, 1364 (1959)

182. J. Dobaczewski, H. Flocard, J. Treiner, Nucl. Phys. A 422, 103 (1984)

183. S. Wuenschel, H. Zheng, K. Hagel, B. Meyer, M. Barbui, E.J. Kim, G. Roepke, J.B. Natowitz, Phys. Rev. C 90, 011601 (2014). https://doi.org/10.1103/PhysRevC.90.011601, [arXiv:1404.4303 [nucl-ex]]

184. L.G. Cao, G. Colo, H. Sagawa, Phys. Rev. C 81, 044302 (2010). https://doi.org/10.1103/PhysRevC.81.044302

185. A.P. Tonchev, N. Tsoneva, C. Bhatia, C.W. Arnold, S. Goriely, S.L. Hammond, J.H. Kelley, E. Kwan, H. Lenske, J. Piekarewicz et al., Phys. Lett. B 773, 20 (2017). https://doi.org/10.1016/j.physletb. 2017.07.062, [arXiv:1708.03522 [nucl-ex]]

186. A.P. Tonchev et al., EPJ Web Conf. 178, 04003 (2018)

187. J. Dong, W. Zuo, J. Gu, Phys. Rev. C 91, 034315 (2015)

188. B.-J. Cai, B.-A. Li, Phys. Rev. C 93, 014619 (2016)

189. P. Danielewicz, J. Lee, Nucl. Phys. A 818, 36 (2009)

190. P. Danielewicz, J. Lee, Nucl. Phys. A 922, 1 (2014)

191. E.E. Saperstein, S.S. Pankratov, M.V. Zverev, M. Baldo, U. Lombardo, Phys. At. Nucl. 72, 1121 (2009)

192. A. Repko, J. Kvasil, V.O. Nesterenko, P.-G. Reinhard, Eur. Phys. J. A 53, 221 (2017)

193. C. Mahaux, R. Sartor, Nucl. Phys. A 528, 253 (1991). https://doi. org/10.1016/0375-9474(91)90090-S

194. J. Meng, K. Sugawara-Tanabe, S. Yamaji, A. Arima, Phys. Rev. C 59, 154 (1999)

195. S.-G. Zhou, J. Meng, P. Ring, Phys. Rev. Lett. 91, 262501 (2003)

196. W. Koepf, P. Ring, Z. Phys, Z. Phys. A 339, 81 (1991)

197. A. Baran, Phys. Rev. C 61, 024316 (2000)

198. G. Accorto, P. Brandolini, F. Marino, A. Porro, A. Scalesi, G. Colò, X. Roca-Maza, E. Vigezzi, Phys. Rev. C 101, 024315 (2020)

199. N. Tsoneva, H. Lenske, Phys. Rev. C 77, 024321 (2008)

200. G. Audi, M. Wang, A.H. Wapstra, F.G. Kondev, M. MacCormick, X. Xu, Nucl. Data Sheets 120, 1 (2014)

201. A. Krasznahorkay et al., Phys. Rev. Lett. 82, 3216 (1999)

202. A. Krasznahorkay et al., AIP Conf. Proc. 610, 751 (2002)

203. G.G. Adamian, L.A. Malov, N.V. Antonenko, R.V. Jolos, Phys. Rev. C 97, 034308 (2018)

204. G.G. Adamian, N.V. Antonenko, W. Scheid, Eur. Phys. J. A 41, 235 (2009)

205. G.G. Adamian, N.V. Antonenko, W. Scheid, Clusters in Nuclei Vol.2, C. Beck (ed.) Lecture Notes in Physics 848, 165, SpringerVerlag, Heidelberg (2012)

206. V.V. Volkov, Izv. Akad. Nauk SSSR, Ser. Fiz. 50, 1879 (1986)

207. N.V. Antonenko, E.A. Cherepanov, A.K. Nasirov, V.B. Permjakov, V.V. Volkov, Phys. Lett. B 319, 425 (1993)

208. N.V. Antonenko, E.A. Cherepanov, A.K. Nasirov, V.B. Permjakov, V.V. Volkov, Phys. Rev. C 51, 2635 (1995)

209. G.G. Adamian, N.V. Antonenko, S.P. Ivanova, W. Scheid, Nucl. Phys. A 646, 29 (1999)

210. G.G. Adamian, N.V. Antonenko, W. Scheid, V.V. Volkov, Nucl. Phys. A 633, 409 (1998)

211. G.G. Adamian, N.V. Antonenko, W. Scheid, V.V. Volkov, Nuovo Cimento A 110, 1143 (1997)

212. G.G. Adamian, N.V. Antonenko, W. Scheid, Nucl. Phys. A 678, $24(2000)$

213. G.G. Giardina, S. Hofmann, A.I. Muminov, A.K. Nasirov, Eur. Phys. J. A 8, 205 (2000)
214. G.G. Giardina, F. Hanappe, A.I. Muminov, A.K. Nasirov, L. Stuttgé, Nucl. Phys. A 671, 165 (2000)

215. A.K. Nasirov et al., Nucl. Phys. A 671, 342 (2005)

216. H.Q. Zhang et al., Phys. Rev. C 81, 034611 (2010)

217. A.K. Nasirov, G. Mandaglio, G.G. Giardina, A. Sobiczewski, A.I. Muminov, Phys. Rev. C 84, 044612 (2011)

218. Z.H. Liu, J.D. Bao, Phys. Rev. C 74, 057602 (2006)

219. N. Wang, J. Tian, W. Scheid, Phys. Rev. C 84, 061601(R) (2011)

220. N. Wang, E.G. Zhao, W. Scheid, S.G. Zhou, Phys. Rev. C 85, 041601(R) (2012)

221. N. Wang, E.G. Zhao, W. Scheid, Phys. Rev. C 89, 037601 (2014)

222. L. Zhu, Z.Q. Feng, C. Li, F.S. Zhang, Phys. Rev. C 90, 014612 (2014)

223. Z.Q. Feng, G.M. Jin, J.Q. Li, W. Scheid, Phys. Rev. C 76, 044606 (2007)

224. A.S. Zubov, G.G. Adamian, N.V. Antonenko, S.P. Ivanova, W. Scheid, Phys. Rev. C 68, 014616 (2003)

225. G.G. Adamian, N.V. Antonenko, W. Scheid, Phys. Rev. C 69 , 011601(R) (2004)

226. G.G. Adamian, N.V. Antonenko, W. Scheid, Phys. Rev. C 69, 014607 (2004)

227. G.G. Adamian, N.V. Antonenko, W. Scheid, Phys. Rev. C 69, 044601 (2004)

228. G.G. Adamian, N.V. Antonenko, W. Scheid, A.S. Zubov, Phys. Rev. C 78, 044605 (2008)

229. G.G. Adamian, N.V. Antonenko, H. Lenske, Nucl. Phys. A 970, 22 (2018)

230. P. Möller, A.J. Sierk, T. Ichikawa, H. Sagawa, At. Data Nucl. Data Tables 109-110, 1 (2016)

231. G.A. Lalazissis, J. Konig, P. Ring, Phys. Rev. C 55, 540 (1997)

232. N. Van Giai, Ch. Stoyanov, V.V. Voronov, Phys. Rev. C 57, 1204 (1998)

233. A.P. Severyukhin, V.V. Voronov, N. Van Giai, Eur. Phys. J. A 22, 397 (2004)

234. N. Tsoneva, H. Lenske, C. Stoyanov, Phys. Lett. B 586, 213 (2004)

235. N. Tsoneva, H. Lenske, A.I.P. Conf, Proc. 1090, 409 (2009)

236. N.N. Arsenyev, A.P. Severyukhin, V.V. Voronov, N. Van Giai, Phys. Rev. C 95, 054312 (2017)

237. V.V. Pashkevich, A.Y. Rusanov, Nucl. Phys. A 810, 77 (2008)

238. M.J. Martin, Nucl. Data Sheets 63, 723 (1991)

239. M.J. Martin, Nucl. Data Sheets 70, 315 (1993)

240. E. Litvinova, P. Ring, Phys. Rev. C 73, 044328 (2006)

241. E. Vigezzi, J. Phys: Conf. Ser. 981, 012007 (2018)

242. E. Litvinova, P. Ring, V. Tselyaev, Phys. Rev. C 75, 064308 (2007)

243. E. Litvinova, A.V. Afanasjev, Phys. Rev. C 84, 014305 (2011)

244. E. Litvinova, Phys. Rev. C 85, 021303 (2012)

245. D. Tarpanov, J. Dobaczewski, J. Toivanen, B.G. Carlsson, Phys. Rev. Lett. 113, 252501 (2014)

246. A.V. Afanasjev, E. Litvinova, Phys. Rev. C 92, 044317 (2015)

247. L.-G. Cao, H. Sagawa, P.F. Bortignon, Phys. Rev. C 89, 044314 (2014)

248. A. Idini, F. Barranco, E. Vigezzi, Phys. Rev. C 85, 014331 (2012)

249. A. Idini, G. Potel, F. Barranco, E. Vigezzi, R.A. Broglia, Phys. Rev. C 92, 031304 (2015)

250. T. Lesinski, T. Duguet, K. Bennaceur, J. Meuer, Eur. Phys. J. A 40, 121 (2009)

251. G. Gori, F. Ramponi, F. Barranco, P.F. Bortignon, R.A. Broglia, G. Colò, E. Vigezzi, Phys. Rev. C 72, 011302 (2005)

252. T. Duguet, T. Lesinski, K. Hebeler, A. Schwenk, Mod. Phys. Lett. 25, 1989 (2010)

253. N.V. Antonenko, L.A. Malov, Izv. RAN, Ser. Phys. 78, 1402 (2014)

254. R.V. Jolos, L.A. Malov, N.Y. Shirikova, A.V. Sushkov, J. Phys. G 38, 115103 (2011)

255. A. Sobiczewski, K. Pomorski, Prog. Part. Nucl. Phys. 58, 292 (2007) 
256. M. Rashdan, Phys. Rev. C 63, 044303 (2001)

257. K. Rutz et al., Phys. Rev. C 56, 238 (1997)

258. M. Bender, K. Rutz, P.-G. Reinhard, J.A. Maruhn, W. Greiner, Phys. Rev. C 60, 034304 (1999)

259. A.T. Kruppa et al., Phys. Rev. C 61, 034313 (2000)

260. T. Sil et al., Phys. Rev. C 69, 044315 (2004)

261. S. Ćwiok, J. Dobaczewski, P.-H. Heenen, P. Magierski, W. Nazarewicz, Nucl. Phys. A 611, 211 (1996)

262. M. Bender, W. Nazarewicz, P.-G. Reinhard, Phys. Lett. B 515, 42 (2001)

263. A. Parkhomenko, A. Sobiczewski, Acta Phys. Pol., B 36, 3115 (2005)

264. A. Parkhomenko, A. Sobiczewski, Acta Phys. Pol. B 35, 2447 (2004)

265. R. Smolańczuk, Phys. Rev. C 56, 812 (1997)

266. S. Liran, A. Marinov, N. Zeldes, Phys. Rev. C 62, 047301 (2000)

267. S. Liran, A. Marinov, N. Zeldes, Phys. Rev. C 63, 017302 (2000)

268. S. Liran, A. Marinov, N. Zeldes, Phys. Rev. C 66, 024303 (2002)

269. S. Liran, A. Marinov, N. Zeldes, arXiv:nuclth/0102055 (2001)

270. X. Viñas, M. Centelles, X. Roca-Maza, M. Warda, AIP Conf. Proc. 1491, 101 (2012)

271. L.-W. Chen, C.M. Ko, B.-A. Li, Phys. Rev. C 72, 064309 (2005)

272. C.J. Horowitz, J. Piekarewicz, Phys. Rev. Lett. 86, 5647 (2001)

273. V.Y. Denisov, V.A. Nesterov, Phys. At. Nucl. 65, 814 (2002)

274. M.K. Gaidarov, A.N. Antonov, P. Sarriguren, E. Moya de Guerra, Phys. Rev. C 84, 034316 (2011)

275. M.K. Gaidarov, A.N. Antonov, P. Sarriguren, E. Moya de Guerra, J. Phys: Conf. Ser. 533, 012016 (2014)

276. M. Warda, M. Centelles, X. Vinas, X. Roca-Maza, Phys. Rev. C 89, 064302 (2014)

277. G. Scamps, D. Lacroix, G.G. Adamian, N.V. Antonenko, Phys. Rev. C 88, 064327 (2013)

278. G.G. Adamian, N.V. Antonenko, L.A. Malov, G. Scamps, D. Lacroix, Phys. Rev. C 90, 034322 (2014)

279. G. Dahlquist, A. Björck, Numerical Methods (Prentice-Hall, Engelwoods Cliffs, 1974)

280. R.N. Bernard, M. Anguiano, Nucl. Phys. A 953, 32 (2016)

281. N. Tsoneva, H. Lenske, Phys. Lett. B 695, 174 (2011)

282. H. De Vries, C.W. De Jager, C. De Vries, At. Data Nucl. Data Tables 36, 495 (1987)

283. S.A. Fayans, JETP Lett. 68, 169 (1998)

284. A.V. Smirnov, S.V. Tolokonnikov, S.A. Fayans, Sov. J. Nucl. Phys. 48, 995 (1988)

285. S.V. Tolokonnikov, I.N. Borzov, M. Kortelainen, Y.S. Lutostansky, E.E. Saperstein, J. Phys. G 42, 075102 (2015)

286. E. Kroemer, S. Tolokonnikov, S. Fayans, D. Zawischa, Phys. Lett. B 363, 12 (1995)

287. D. Horen, G. Satchler, S. Fayans, E. Trykov, Nucl. Phys. A 600, 193 (1996)

288. S.V. Tolokonnikov, E.E. Saperstein, Phys. At. Nucl. 73, 1684 (2010)

289. J. Erler, P. Klupfel, P.G. Reinhard, Phys. Rev. C 82, 044307 (2010). https://doi.org/10.1103/PhysRevC.82.044307, [arXiv:1009.0624 [nucl-th]]

290. B. Friedman, V.R. Pandharipande, Nucl. Phys. A 361, 502 (1981)

291. R.B. Wiringa, V. Fiks, A. Fabrocini, Phys. Rev. C 38, 1010 (1988)

292. S.A. Fayans, D. Zawischa, Int. J. Mod. Phys. B 15, 1684 (2001). https://doi.org/10.1142/S0217979201006203, [arXiv:nucl-th/0009034 [nucl-th]]

293. E.E. Saperstein, S.V. Tolokonnikov, Phys. Atom. Nucl. 74, 1277 (2011)

294. I.N. Borzov, E.E. Saperstein, S.V. Tolokonnikov, Phys. Atom. Nucl. 71, 469 (2008)

295. I.N. Borzov, E.E. Saperstein, S.V. Tolokonnikov, G. Neyens, N. Severijns, Eur. Phys. J. A 45, 159 (2010)
296. S.V. Tolokonnikov, S. Kamerdzhiev, D. Voitenkov, S. Krewald, E.E. Saperstein, Phys. Rev. C 84, 064324 (2011)

297. S.V. Tolokonnikov, S. Kamerdzhiev, S. Krewald, E.E. Saperstein, D. Voitenkov, Eur. Phys. J. A 48, 70 (2012)

298. S. Kamerdzhiev, S. Krewald, S. Tolokonnikov, E.E. Saperstein, D. Voitenkov, EPJ Web Conf. 38, 10002 (2012)

299. S.V. Tolokonnikov, S. Kamerdzhiev, S. Krewald, E.E. Saperstein, D. Voitenkov, EPJ Web Conf. 38, 04002 (2012)

300. N.V. Gnezdilov, I.N. Borzov, E.E. Saperstein, S.V. Tolokonnikov, Phys. Rev. C 89, 034304 (2014)

301. P.G. Reinhard, W. Nazarewicz, Phys. Rev. C 95, 064328 (2017). https://doi.org/10.1103/PhysRevC.95.064328, [arXiv:1704.07430 [nucl-th]]

302. R.A. Kuzyakin, V.V. Sargsyan, G.G. Adamian, N.V. Antonenko, E.E. Saperstein, S.V. Tolokonnikov, Phys. Rev. C 85, 034612 (2012)

303. T.M. Shneidman, G.G. Adamian, N.V. Antonenko, R.V. Jolos, W. Scheid, Phys. Rev. C 67, 014313 (2003)

304. J. Meng, I. Tanihata, S. Yamaji, Phys. Lett. B 419, 1 (1998)

305. G.G. Adamian et al., Int. J. Mod. Phys. E 5, 191 (1996)

306. V.V. Sargsyan, G.G. Adamian, N.V. Antonenko, W. Scheid, H.Q. Zhang, Phys. Rev. C 84, 064614 (2011)

307. M. Bender, G.F. Bertsch, P.-H. Heenen, Phys. Rev. C 73, 034322 (2006)

308. J.-P. Delaroche, M. Girod, J. Libert, H. Goutte, S. Hilaire, S. Péru, N. Pillet, G.F. Bertsch, Phys. Rev. C 81, 014303 (2010)

309. K. Hagino, N.W. Lwin, M. Yamagami, Phys. Rev. C 74, 017310 (2006)

310. W.D. Myers, W.J. Światecki, Phys. Rev. C 58, 3368 (1998)

311. I. Dutt, R.K. Puri, Phys. Rev. C 81, 047601 (2010)

312. P. Bonche, H. Flocard, P.-H. Heenen, Comput. Phys. Commun. 171, 49 (2005)

313. G.F. Bertsch, C.A. Bertulani, W. Nazarewicz, N. Schunck, M.V. Stoitsov, Phys. Rev. C 79, 034306 (2009)

314. E. Chabanat, P. Bonche, P. Haensel et al., Nucl. Phys. A 635, 231 (1998)

315. J. Bartel, P. Quentin, M. Brack, C. Guet, H.-B. Hakansson, Nucl. Phys. A 386, 79 (1982)

316. A. Saxena, V.S. Ramamurthy, Pramana J. Phys. 27, 15 (1986)

317. B. Nerlo-Pomorska, K. Pomorski, Z. Phys. A 348, 169 (1994)

318. B. Nerlo-Pomorska, B. Mach, Atom. Data Nucl. Data Tables 60, 287 (1995)

319. B.A. Brown, C.R. Bronk, P.E. Hodgson, J. Phys. G 10, 1683 (1984)

320. G. Scamps, D. Lacroix, Phys. Rev. C 88, 044310 (2013)

321. N. Tajima, Progr. Theor. Phys. Suppl. 142, 265 (2001)

322. P. Sarriguren, M.K. Gaidarov, E. Moya de Guerra, A.N. Antonov, Phys. Rev. C 76, 044322 (2007)

323. D.T. Khoa, W. von Oertzen, A.A. Ogloblin, Nucl. Phys. A 602, 98 (1996)

324. S.G. Zhou, J. Meng, P. Ring, E.G. Zhao, Phys. Rev. C 82, 011301(R) (2010)

325. L.L. Li, J. Meng, P. Ring, E.G. Zhao, S.G. Zhou, Phys. Rev. C 85, $024312(2012)$

326. J.C. Pei, Y.N. Zhang, F.R. Xu, Phys. Rev. C 87, 051302(R) (2013)

327. X.X. Sun, J. Zhao, S.G. Zhou, Phys. Lett. B 785, 530 (2018)

328. H. Nakada, K. Takayama, Phys. Rec. C 98, 011301(R) (2018)

329. D. Pena Arteaga, E. Khan, P. Ring, Phys. Rev. C 79, 034311 (2009)

330. V.V. Sargsyan, G.G. Adamian, N.V. Antonenko, W. Scheid, Eur. Phys. J. A 45, 125 (2010)

331. V.V. Sargsyan, G.G. Adamian, N.V. Antonenko, W. Scheid, H.Q. Zhang, Phys. Rev. C 86, 034614 (2012)

332. W.M. Seif, N.V. Antonenko, G.G. Adamian, H. Anwer, Phys. Rev. C 96, 054328 (2017)

333. W.M. Seif, M. Shalaby, M.F. Alkarshy, Phys. Rev. C 84, 064508 (2011) 
334. A.V. Afanasjev, O. Abdurazakov, Phys. Rev. C 88, 014320 (2013)

335. A.V. Afanasjev, J. Phys. G 42, 034002 (2015)

336. M. Leino, F.P. Herzberg, Annu. Rev. Nucl. Part. Sci. 54, 175 (2004)

337. R.-D. Herzberg, P.T. Greenlees, Prog. Part. Nucl. Phys. 61, 674 (2008)

338. D. Ackermann, Ch. Theisen, Phys. Scr. 92, 083002 (2017)

339. F.P. Hessberger et al., Eur. Phys. J. A 22, 417 (2004)

340. F.P. Hessberger et al., Eur. Phys. J. A 30, 561 (2006)

341. F.P. Hessberger et al., Eur. Phys. J. A 12, 57 (2001)

342. B. Streicher et al., Eur. Phys. J. A 45, 275 (2010)

343. A. Chatillon et al., Eur. Phys. J. A 30, 397 (2006)

344. F.P. Hessberger et al., Eur. Phys. J. A 26, 233 (2005)

345. F.P. Hessberger et al., Eur. Phys. J. A 41, 145 (2009)

346. F.P. Hessberger et al., Eur. Phys. J. A 43, 175 (2010)

347. S. Antalic et al., Eur. Phys. J. A 43, 35 (2010)

348. S.K. Tandel et al., Phys. Rev. C 82, 041301 (2010)

349. S. Hofmann, G. Münzenberg, Rev. Mod. Phys. 72, 737 (2000)

350. V.G. Soloviev, A.V. Sushkov, N.Y. Shirikova, Sov. J. Nucl. Phys. 54, 748 (1991)

351. F.A. Gareev, S.P. Ivanova, L.A. Malov, V.G. Soloviev, Nucl. Phys. A 171, 434 (1971)

352. S.G. Nilsson, I. Ragnarsson, Shapes and shells in nuclear structure (Cambridge University Press, Cambridge, 1995)

353. S. Ćwiok, S. Hofmann, W. Nazarewicz, Nucl. Phys. A 573, 356 (1994)

354. G.G. Adamian, N.V. Antonenko, W. Scheid, Phys. Rev. C 81, 024320 (2010)

355. G.G. Adamian, N.V. Antonenko, W. Scheid, Acta Phys. Pol. B 40, 759 (2009)

356. L. Bonneau, P. Quentin, P. Möller, Phys. Rev. C 76, 024320 (2007)

357. P. Ring, P. Schuck, The nuclear many-body problem (SpringerVerlag, New York, 1980)

358. S. Goriely, N. Chamel, J. M. Pearson, Phys. Rev. Lett. 102, 152503 (2009); http://www-astro.ulb.ac.de/Html/masses.html

359. X.T. He, Z.Z. Ren, S.X. Liu, E.G. Zhao, Nucl. Phys. A 817, 45 (2009)

360. M.V. Stoitsov, J. Dobaczewski, W. Nazarewicz, P. Ring, Comput. Phys. Commun. 167, 43 (2005)

361. M.V. Stoitsov, J. Dobaczewski, W. Nazarewicz, S. Pittel, D.J. Dean, Phys. Rev. C 68, 054312 (2003)

362. E. Chabanat, P. Bonche, P. Haensel, J. Meyer, R. Schaeffer, Nucl. Phys. A 643, 441 (1998)

363. http://www.nndc.bnl.gov/ensdf/

364. F.P. Hessberger, Eur. Phys. J. D 45, 33 (2007)

365. Y. Shi, D.E. Ward, B.G. Carlsson, J. Dobaczewski, W. Nazarewicz, I. Ragnarsson, D. Rudolph, Phys. Rev. C 90, 014308 (2014)

366. M. Kortelainen, J. McDonnell, W. Nazarewicz, P.G. Reinhard, J. Sarich, N. Schunck, M.V. Stoitsov, S.M. Wild et al., Phys. Rev. C 85, 024304 (2012)

367. Y. Shi, J. Dobaczewski, P.T. Greenlees, Phys. Rev. C 89, 034309 (2014)

368. P. Klupfel, P.-G. Reinhard, T.J. Burvenich, J.A. Maruhn, Phys. Rev. C 79, 034310 (2009)

369. P.-G. Reinhard, code SKYAX

370. M. Bender, K. Rutz, P.-G. Reinhard, J.A. Maruhn, Eur. Phys. J. A 8, 59 (2000)

371. G. Scamps, D. Lacroix, Phys. Rev. C 87, 014605 (2013)

372. R.D. Ratna-Raju, J.P. Drayer, K.T. Mecht, Nucl. Phys. A 202, 433 (1973)

373. A.V. Karpov, V.I. Zagrebaev, Y.M. Palazuela, L.F. Ruiz, W. Greiner, Int. J. Mod. Phys. E 21, 1250013 (2012)

374. P. Sarriguren, Phys. Rev. C 100, 014309 (2019)

375. P. Sarriguren, J. Phys. G 47, 125107 (2020)

376. D. Vautherin, Phys. Rev. C 7, 296 (1973)
377. K. Langanke, G. Matrinez-Pinedo, Rev. Mod. Phys. 75, 819 (2003)

378. A.P. Severyukhin, N.N. Arsenyev, I.N. Borsov, E.O. Sushenok, Phys. Rev. C 95, 034314 (2017)

379. G. Lorusso et al., Phys. Rev. Lett. 114, 192501 (2015)

380. H. Mach, D. Jerrestam, B. Fogelberg, M. Hellström, J.P. Omtvedt, K.I. Erokhina, V.I. Isakov, Phys. Rev. C 51, 500 (1995)

381. R. Dunlop et al., Phys. Rev. C 93, 062801(R) (2016)

382. A. Jungclaus et al., Phys. Rev. C 94, 024303 (2016)

383. I.N. Borsov, Phys. Rev. C 71, 065801 (2005)

384. I.N. Borsov, J.J. Cuenca-Garsia, K. Langanke, G. MartinezPinedo, F. Montes, Nucl. Phys. A 814, 159 (2008)

385. Y.F. Niu, G. Colò, M. Brenna, P.F. Bortignon, J. Meng, Phys. Rev. C 85, 034314 (2012)

386. Y.F. Niu, Z.M. Niu, G. Colò, E. Vigezi, Phys. Lett. B 780, 325 (2018)

387. Y.F. Niu, Z.M. Niu, G. Colò, E. Vigezi, Phys. Rev. Lett. 114, $142501(2015)$

388. A.P. Severyukhin, J. Margueron, I.N. Borzov, N. Van Giai, Phys. Rev. C 91, 034322 (2015)

389. H.-T. Janka, K. Langanke, A. Marek, G. Martinez-Pinedo, B. Mueller, Phys. Rep. 442, 38 (2007)

390. A.A. Dzhioev, A.I. Vdovin, V.Y. Ponomarev, J. Wambach, K. Langanke, G. Martinez-Pinedo, Phys. Rev. C 81, 015804 (2010)

391. A.A. Dzhioev, K. Langanke, G. Martinez-Pinedo, A.I. Vdovin, Ch. Stoyanov, Phys. Rev. C 101, 025805 (2020)

392. R. Titus, E.M. Ney, R.G.T. Zegers, D. Bazin, J. Belarge, P.C. Bender, B.A. Brown, C.M. Campbell, B. Elman, J. Engel, A. Gade, B. Gao, E. Kwan, S. Lipschutz, B. Longfellow, E. Lunderberg, T. Mijatovic, S. Noji, J. Pereira, J. Schmitt, C. Sullivan, D. Weisshaar, J.C. Zamora, Phys. Rev. C 100, 045805 (2019)

393. J.C. Zamora, R.G.T. Zegers, S.M. Austin, D. Bazin, B.A. Brown, P.C. Bender, H.L. Crawford, J. Engel, A. Falduto, A. Gade, P. Gastis, B. Gao, T. Ginter, C.J. Guess, S. Lipschutz, B. Longfellow, A.O. Macchiavelli, K. Miki, E. Ney, S. Noji, J. Pereira, J. Schmitt, C. Sullivan, R. Titus, D. Weisshaar, Phys. Rev. C 100, 032801(R) (2019)

394. V.V. Pashkevich, Nucl. Phys. A 133, 400 (1969)

395. P. Möller, J.R. Nix, in Proceedings of the Third IAEA Symposium on Physics and Chemistry of Fission, Rochester, New York, 13-17 August 1973, pp. 103-140

396. K. Rutz, J.A. Maruhn, P.G. Reinhard, W. Greiner, Nucl. Phys. A 590, 680 (1995)

397. L.M. Robledo, M. Warda, Int. J. Mod. Phys. E 17, 204 (2008)

398. M. Kowal, P. Jachimowicz, A. Sobiczewski, Phys. Rev. C 82, 014303 (2010)

399. Z.P. Li, T. Nikšić, D. Vretenar, P. Ring, J. Meng, Phys. Rev. C 81, 064321 (2010)

400. H. Abusara, A.V. Afanasjev, P. Ring, Phys. Rev. C 82, 034303 (2010)

401. A. Staszczak, A. Baran, W. Nazarewicz, Int. J. Mod. Phys. E 20, $552(2011)$

402. H. Abusara, A.V. Afanasjev, P. Ring, Phys. Rev. C 85, 024314 (2012)

403. V. Prassa, T. Nikšić, G.A. Lalazissis, D. Vretenar, Phys. Rev. C 86, 024317 (2012)

404. G. Royer, M. Jaffre, D. Moreau, Phys. Rev. C 86, 044326 (2012)

405. B.N. Lu, E.G. Zhao, S.G. Zhou, Phys. Rev. C 85, 011301(R) (2012)

406. M. Warda, J.L. Egido, Phys. Rev. C 86, 014322 (2012)

407. B.N. Lu, J. Zhao, E.G. Zhao, S.G. Zhou, Phys. Rev. C 89, 014323 (2014)

408. S.E. Agbemava, A.V. Afanasjev, D. Ray, P. Ring, Phys. Rev. C 95, 054324 (2017)

409. S.E. Agbemava, A.V. Afanasjev, Phys. Rev. C 96, 024301 (2017) 
410. T. Bürvenich, M. Bender, J.A. Maruhn, P.G. Reinhard, Phys. Rev. C 96, 024301 (2017)

411. G. Scamps, C. Simenel, Nature 564, 382 (2018)

412. X. Meng, B.N. Lu, S.G. Zhou, Sci. China-Phys. Mech. Astron. 63, 212011 (2020)

413. C. Simenel, A.S. Umar, Prog. Part. Nucl. Phys. 103, 19 (2018)

414. R. Rodríguez-Guzmán, L.M. Robledo, Phys. Rev. C 98, 034308 (2018)

415. H. Paşca, A.V. Andreev, G.G. Adamian, N.V. Antonenko, Nucl. Phys. A 977, 1 (2018)

416. Qing-Zhen Chai, Wei-Juan Zhao, Hua-Lei Wang, Min-Liang Liu, Fu-Rong Xu, Prog. Theor. Expt. Phys. 2018, 053 D02 (2018)

417. Q.-Z. Chai, W.-J. Zhao, M.-L. Liu, H.-L. Wang, Chin. Phys. C 42, 054101 (2018)

418. H. Paşca, A.V. Andreev, G.G. Adamian, N.V. Antonenko, Y. Kim, Phys. Rev. C 93, 054602 (2016)

419. H. Paşca, A.V. Andreev, G.G. Adamian, N.V. Antonenko, Phys. Rev. C 97, 034621 (2018)

420. H. Paşca, A.V. Andreev, G.G. Adamian, N.V. Antonenko, Phys. Rev. C 101, 064604 (2020)

421. A.V. Afanasjev, S.E. Agbemava, A. Gyawali, Phys. Lett. B 782, $533(2018)$

422. M. Bhuyan, B.V. Carlson, S.K. Patra, R.K. Gupta, Phys. Rev. C 100, 054312 (2019)

423. G. Scamps, C. Simenel, Phys. Rev. C 100, 041602(R) (2019)

424. J. Dobaczewski, arXiv:1910.03924 [nucl-th]

425. J. Zhao, T. Nikšić, D. Vretenar, S.G. Zhou, Phys. Rev. C 99, 014618 (2019)

426. S.E. Agbemava, A.V. Afanasjev, A. Taninah, A. Gyawali, Phys. Rev. C 99, 034316 (2019)

427. J. Zhao, J. Xiang, Z.P. Li, T. Nikšić, D. Vretenar, S.G. Zhou, Phys. Rev. C 99, 054613 (2019)

428. Z. Zhi, A.V. Afanasjev, Z.P. Li, J. Meng, Phys. Rev. C 99, 064316 (2019)

429. Z.M. Wang, W.J. Zhu, X. Zhu, C.L. Zhong, T.S. Fan, Commun. Theor. Phys. 71, 417 (2019)

430. Q.Z. Chai, W.J. Zhao, H.L. Wang, Commun. Theor. Phys. 71, 67 (2019)

431. J. Zhao, B.N. Lu, E.G. Zhao, S.G. Zhou, Phys. Rev. C 95, 014320 (2017)

432. J. Zhao, B.N. Lu, D. Vretenar, E.G. Zhao, S.G. Zhou, Phys. Rev. C 91, 014321 (2015)

433. J. Zhao, B.N. Lu, E.G. Zhao, S.G. Zhou, Phys. Rev. C 86, 057304 (2012)

434. C. Liu et al., Phys. Rev. Lett. 116, 112501 (2016)

435. X.C. Chen et al., Phys. Rev. C 94, 021301(R) (2016)

436. B.N. Lu, E.G. Zhao, S.G. Zhou, Phys. Rev. C 84, 014328 (2011)

437. B.N. Lu, E. Hiyama, H. Sagawa, S.G. Zhou, Phys. Rev. C 89, 044307 (2014)

438. B.N.L. Zhao, T. Nikšić, D. Vretenar, Phys. Rev. C 92, 064315 (2015)

439. J. Zhao, B.N. Lu, T. Nikšić, D. Vretenar, S.G. Zhou, Phys. Rev. C 93, 044315 (2016)

440. S. Karatzikos, A.V. Afanasjev, G.A. Lalazissis, P. Ring, Phys. Lett. B 689, 72 (2010)

441. Z. Ren, Phys. Rev. C 65, 051304(R) (2002)
442. Z. Ren, F. Tai, D.H. Chen, Phys. Rev. C 66, 064306 (2002)

443. L. Geng, H. Toki, J. Meng, Prog. Theor. Phys. 113, 785 (2005)

444. H.F. Zhang, Y. Gao, N. Wang, J.Q. Li, E.G. Zhao, G. Royer, Phys. Rev. C 85, 014325 (2012)

445. S. Goriely, N. Chamel, J.M. Pearson, Phys. Rev. C 88, 024308 (2013)

446. V. Prassa, T. Nikšić, D. Vretenar, Phys. Rev. C 88, 044324 (2013)

447. X.W. Xia, Y. Lim, P.W. Zhao, H.Z. Liang, X.Y. Qu, Y. Chen, H. Liu, L.F. Zhang, S.Q. Zhang, Y. Kim, J. Meng, Atomic Data Nucl. Data Tables 121-122, 1 (2018)

448. M. Shi, Z.M. Niu, H.Z. Liang, Chin. Phys. C 43, 074104 (2019)

449. A. Staszczak, A. Baran, W. Nazarewicz, Phys. Rev. C 87, 024320 (2013)

450. A. Baran, M. Kowal, P.-G. Reinhard, L. Robledo, A. Staszczak, M. Warda, Nucl. Phys. A 944, 442 (2015)

451. J. Sadhukhan, K. Mazurek, A. Baran, J. Dobaczewski, W. Nazarewicz, J.A. Sheikh, Phys. Rev. C 88, 064314 (2013)

452. J. Sadhukhan, J. Dobaczewski, W. Nazarewicz, J.A. Sheikh, A. Baran, Phys. Rev. C 90, 061304 (2014)

453. G. Audi, O. Bersillon, J. Blachot, A.H. Wapstra, Nucl. Phys. A 729, 3 (2003)

454. R. Jodon, M. Bender, K. Bennaceur, J. Meyer, Phys. Rev. C 94, 024335 (2016)

455. W. Ryssens, M. Bender, K. Bennaceur, P.-H. Heenen, J. Meyer, Phys. Rev. C 99, 044315 (2019)

456. J. Berger, M. Girod, D. Gogny, Comput. Phys. Commun. 63, 365 (1991)

457. M. Bender, K. Rutz, P.-G. Reinhard, J.A. Maruhn, Eur. Phys. J. A 7, 467 (2000)

458. S. Goriely, M. Samyn, J.M. Pearson, Phys. Rev. C 75, 064312 (2007)

459. N. Nikolov, N. Schunck, W. Nazarewicz, M. Bender, J. Pei, Phys. Rev. C 83, 034305 (2011)

460. M. Brack, C. Guet, H.-B. Hakansson, Phys. Rep. 123, 275 (1985)

461. H. Paşca, A.V. Andreev, G.G. Adamian, N.V. Antonenko, Eur. Phys. J. A 52, 369 (2016)

462. H. Paşca, A.V. Andreev, G.G. Adamian, N.V. Antonenko, Phys. Lett. B 760, 800 (2016)

463. M. Warda, L.M. Robledo, Phys. Rev. C 84, 044608 (2011)

464. M. Warda, A. Zdeb, L.M. Robledo, Phys. Rev. C 98, 041602 (2018)

465. C. Ishizuka, X. Zhang, M.D. Usang, F.A. Ivanyuk, S. Chiba, Phys. Rev. C 101, 011601(R) (2020)

466. M. Albertsson, B.G. Carlsson, T. Dossing, P. Möller, J. Randrup, S. Aberg, Eur. Phys. J. A 56, 46 (2020) 